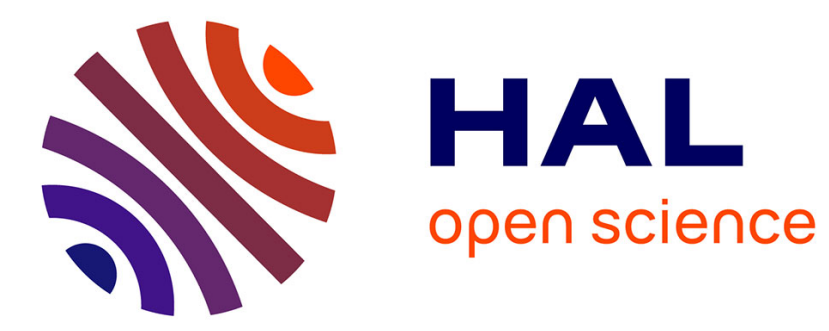

\title{
Tracing the geomagnetic field intensity variations in Upper Mesopotamia during the Pottery Neolithic to improve ceramic-based chronologies
}

\author{
Y Gallet, A Fournier, P W Livermore
}

\section{- To cite this version:}

Y Gallet, A Fournier, P W Livermore. Tracing the geomagnetic field intensity variations in Upper Mesopotamia during the Pottery Neolithic to improve ceramic-based chronologies. Journal of Archaeological Science, 2021, 132, pp.105430. 10.1016/j.jas.2021.105430 . hal-03436220

\author{
HAL Id: hal-03436220 \\ https://hal.science/hal-03436220
}

Submitted on 19 Nov 2021

HAL is a multi-disciplinary open access archive for the deposit and dissemination of scientific research documents, whether they are published or not. The documents may come from teaching and research institutions in France or abroad, or from public or private research centers.
L'archive ouverte pluridisciplinaire HAL, est destinée au dépôt et à la diffusion de documents scientifiques de niveau recherche, publiés ou non, émanant des établissements d'enseignement et de recherche français ou étrangers, des laboratoires publics ou privés. 
1 Tracing the geomagnetic field intensity variations in Upper Mesopotamia during the

2 Pottery Neolithic to improve ceramic-based chronologies

3 Y. Gallet ${ }^{1}$, A. Fournier ${ }^{1}$, P. W. Livermore ${ }^{2}$

$4 \quad{ }^{1}$ Université de Paris, Institut de Physique du Globe de Paris, CNRS, F-75005 Paris, France

$5 \quad{ }^{2}$ School of Earth \& Environment, University of Leeds, Leeds LS2 9JT, UK

7 Abstract

8 The transdimensional Bayesian method AH-RJMCMC applied to archeomagnetic intensity 9 data available in the Balkans and the Near East allows us to estimate the variations in 10 intensity of the geomagnetic field in Upper Mesopotamia during the $7^{\text {th }}$ and $6^{\text {th }}$ millennia BCE 11 (Late Neolithic), with adequate treatment of the dating and intensity uncertainties. The results 12 for the $6^{\text {th }}$ millennium BCE appear particularly interesting because there is enough data to 13 trace rapid geomagnetic field intensity variations, with two century-scale peaks around 5800 $\mathrm{BCE}$ and $5550 \mathrm{BCE}$, associated with rates of changes $(>0.2 \mu \mathrm{T} / \mathrm{year})$ higher than the maximum rate observed in the current geomagnetic field. We show that these variations could help decipher the correlations between different archeological sequences or periodizations established from scattered sites in Upper Mesopotamia. So far documented only from the Balkan data, the intensity peak occurring around 5800 BCE may provide accurate chronological constraints for the Early Halaf phase. New insights are also obtained for the Halaf-Ubaid Transitional phase (end of the $6^{\text {th }}$ millennium BCE), which remains poorly

21 defined from an archeological point of view. The AH-RJMCMC results imply that either the 22 archeointensity data currently available from Upper Mesopotamia document only the 23 beginning of this phase, or that this phase occurred between 5400 and 5200 BCE, shorter 
than often considered. Such preliminary archeological inferences will progress and broaden with the addition of new archeointensity data.

Keywords: archeomagnetic intensity variation, transdimensional Bayesian method, pottery horizons, Late Neolithic, archeological inferences

\section{Introduction}

The end of the Neolithic in the Near East was a period of profound cultural and social transformations probably linked, at least in part, to the definitive adoption of pottery around 7000 BCE and then to its wide regional diffusion and diversification, with technological improvements, throughout the 7th and 6th millennia BCE (e.g. Akkermans and Schwartz, 2003; Nieuwenhuyse et al., 2010; 2013; Tsuneki et al. 2017; Gómez-Bach et al., 2018a; Nieuwenhuyse, 2018a,b). As early as the period known as the Pottery Neolithic or Late Neolithic, ceramic horizons are therefore of great importance for establishing a regional periodization valid for scattered archeological sites, especially in Syria and Iraq, and/or for synchronizing occupation phases between different agricultural settlements in Upper Mesopotamia whose number increased considerably during the 7th and 6th millennia BCE (e.g. Akkermans and Schwartz, 2003; Huot, 2004).

Many questions remain with regard to the meaning and interpretation, in terms of chronological constraints, of the stylistic differences (vessel shapes and decorations) observed between ceramics found in Neolithic sites scattered across Upper Mesopotamia. They are all the more significant as the degree of standardization of ceramic production remains poorly understood for this ancient period, the very notion of regional standardization not even being assured (see the discussions by Hole (2013), Frangipane (2013) and Nieuwenhuyse (2013)). To briefly summarize, it is indeed particularly difficult to disentangle a difference of 
essentially local origin (what Hole (2013) compared to dialect variations in language), possibly associated with a lack of social interaction, rejection or different conservatism between villages, from a difference of broader origin that could reflect changes of techniques and/or preferences, for example through artistic emulation between settlements (recall the beauty and sophistication of the decorations on ceramics from the so-called Halaf period during the 6th millennium BCE; e.g. Akkermans and Schwartz, 2003; Nieuwenhuyse et al., 2013 and references therein). This last option would allow for a regional network of social interactions to be envisaged. Such interactions may have taken the form of trade (e.g. Davidson and McKerrell, 1980; Davidson, 1981; Spataro and Fletcher, 2010), the pottery being a commercial product par excellence, surplus exchanges (Gómez-Bach et al., 2018b), or they may have been associated with the displacement of artisan potters throughout Upper Mesopotamia. Furthermore, the possibility that women played a role in the dissemination of pottery know-how through marriages between people from different localities cannot be ruled out (e.g. Robert, 2010; Forest, 2013).

Dates are crucial here, especially since the history of archeological discoveries and regional geopolitical vicissitudes have led to archeological chronologies with different terminologies established between the east and west of Upper Mesopotamia since the 1930s (e.g. Nieuwenhuyse et al., 2013 and references therein). Nowadays it is very difficult to accurately synchronize these chronologies due to the lack of sufficient radiocarbon dating in many sites, as well as sometimes to the lack of precise description of the ceramic typologies and/or of the archeological contexts in which the pottery was discovered more than half a century ago (a synthesis of available data is provided by Nieuwenhuyse et al. (2013), Nieuwenhuyse (2018a), Gómez-Bach et al. (2018a) and references therein).

This study aims to enable progress in the study of chronologies, by demonstrating the importance of using variations in geomagnetic intensities as chronological markers. Recent 
studies have shown the potential of archeomagnetism for archeological practice, in particular for dating burnt structures found in situ (such as pottery or domestic kilns) and the associated archeological contexts. For the most part, they focused on structures dated from the past two millennia, the archeomagnetic dating relying mainly on the directional geomagnetic field variations (e.g. Gallet et al., 2009; Principe et al., 2018; Korte et al., 2019; Genevey et al., 2021). Thanks to the recent acquisition of numerous new data on geomagnetic field intensities, particularly in the Near East, archeointensities can now also be used to provide chronological constraints, opening a wide field of investigation on ceramic productions (i.e. materials found displaced from the place where they were fired and therefore for which all information from geomagnetic directions is lost; e.g. Genevey et al., 2021). The technique used for this purpose can either rely on a statistical correlation between a result of unknown age and a reference (dated) geomagnetic secular variation curve (e.g. Le Goff et al., 2002; Pavón-Carrasco et al., 2011) or on posterior probability distributions of age values determined by marginalization from a Bayesian approach (e.g. Schnepp et al., 2015; Hervé and Lanos, 2017; Livermore et al., 2018) (see further discussion and comparison between the two methods in Genevey et al., 2021). It should be noted that dating based on a single archeointensity value can lead to poorly constrained results, especially if analyzed in the absence of other archeological, archeomagnetic (i.e. direction-based) or isotopic (radiocarbon) information. However, two studies focusing on time-ordered series of results dated to the 3rd and 2nd millennia BCE in the Near East recently illustrated the contribution of archeointensity alone to archeomagnetic dating (Gallet et al., 2020; Shaar et al., 2020). They both rely on the recent development of a new transdimensional Bayesian technique allowing the construction of regional geomagnetic intensity variation curves, not based on any a priori assumption as to the nature of the geomagnetic variations sought (Livermore et al., 2018; 2021). This method makes it possible to trace fluctuations of highly variable nature both in 
amplitude and duration (from a few decades to several millennia), without the need to seek a compromise through a global regularization parameter that smoothes the entire model. The main objective of our study is to apply this method to the archeomagnetic intensity results

101 obtained in the Balkans dating from the $6^{\text {th }}$ millennium BCE (Fanjat et al., 2013; Kovacheva 102 et al., 2014; Kostadinova-Avramova et al., 2019), to the data from the 7th and 6th millennia 103 BCE currently available in the Near East (Gallet et al., 2015; Yutsis-Akimova et al., 2018a, 104 2018b), and finally, to construct a composite Upper Mesopotamian archeointensity variation curve between $7000 \mathrm{BCE}$ and $5000 \mathrm{BCE}$, with adequate treatment of the dating and intensity uncertainties, assuming that the Balkans and Upper Mesopotamia shared the same secular

107 variation in geomagnetic field intensity during this time interval.

At this stage, our study is intended to illustrate the potential of a method that could later integrate new archeological contexts, with pottery fragments already available in archeological repositories or still to be discovered in the Near East. In addition to the

111 information on the behavior of the geomagnetic field during the 7th and 6th millennia BCE, 112 the results already obtained bring new perspectives to refine the archeological chronologies of 113 the Pottery Neolithic period in the Near East.

\section{Selected datasets and methodology}

\section{2.a Archeointensity datasets}

117 For the Balkans, the results dating from the 6th millennium BCE were obtained in the 118 Paleomagnetism laboratory of Sofia (Bulgaria) under the direction of M. Kovacheva. Mainly 119 deriving from in-situ burnt structures, the Bulgarian dataset was compiled by Kovacheva et al. 120 (2014). More recently, new archeointensity data were obtained by Kostadinova-Avramova et 121 al. (2019), which have therefore also been taken into account in the present work. To these 
122 data, a result obtained in Northern Greece by Fanjat et al. (2013) was also added. In total, the

123 database compiled for the Balkans contains 51 archeointensity values whose dating is based

124 on archeological constraints and/or radiocarbon data (Table S1; more information is available

125 in the associated articles). For simplicity, given the lack of precise information on the

126 probability distributions of radiocarbon dates, we considered below only uniformly

127 distributed age uncertainties. Furthermore, a time-order relationship was applied for a few

128 limited sets of data (Kostadinova-Avramova et al., 2014; 2019; Table S1).

The Upper Mesopotamian data were obtained from groups of pottery fragments sampled in Syria, at Tell Halula (e.g. Molist et al., 2013) and Tell Masaikh (Robert, 2010), and in

131 Northern Iraq, at Yarim Tepe I and II (e.g. Merpert and Munchaev, 1987, 1993a,b; Amirov, 132 1994; 2018). In all cases, these are multi-layered sites that have been the subject of detailed 133 excavations. Each group, which includes a minimum of three fragments from different pottery

134 that gave intensity values satisfying a set of selection criteria using the Triaxe experimental 135 protocol (e.g. Le Goff and Gallet, 2004; Gallet and Le Goff, 2006; Gallet et al., 2020), is 136 directly associated with an archeological level, itself placed in a stratigraphic/temporal 137 sequence. Here we consider the ceramic (chronological) phasing as provided by the 138 archeologists. At Tell Halula, 22 groups of potsherds gave archeointensity values spanning 139 the 7th and 6th millennia BCE, i.e. during the so-called Initial Pottery Neolithic, Early Pottery 140 Neolithic, pre Halaf, proto-Halaf and Halaf cultural phases (Molist et al., 2013; Gallet et al., 141 2015; Nieuwenhuyse et al., 2013; Nieuwenhuyse, 2018a). However, the last centuries of the 142 6th millennium corresponding to the so-called Halaf Ubaid Transitional (HUT) period are not 143 represented in this site, which is instead likely represented at Tell Masaikh with two 144 archeointensity values (Robert et al., 2008; Robert, 2010; Gallet et al., 2015). It is 145 nevertheless important to emphasize the great archeological uncertainties that remain 146 concerning the definition, or even the existence, of the HUT phase (e.g. Campbell, 2007; 
147 Campbell and Fletcher, 2010; Gómez-Bach et al., 2016; Nieuwenhuyse et al., 2016). The 148 Iraqi sites of Yarim Tepe II and I excavated by Soviet archeologists in the 1970s yielded 149 respectively 23 and 9 archeointensity values, from nine and eight different archeological 150 levels (or building horizons). At Yarim Tepe II, the temporal sequence with intensity data 151 ranges from the end of the Early Halaf / beginning of the Middle Halaf to the HUT period 152 (e.g. Amirov, 1994; 2018; Yutsis-Akimova et al., 2018a), while that of Yarim Tepe I partially 153 covers the archaic Hassuna and the standard Hassuna phases dated from the end of the 7th 154 millennium and beginning of the 6th millennium BCE (e.g. Bashilov et al., 1980; Bader, 155 1989; Bernbeck and Nieuwenhuyse, 2013; see also Yutsis-Akimova et al., 2018b). To all 156 these data is added a new Triaxe archeointensity result obtained from a group of potsherds 157 sampled at Tell Begum in Iraqi Kurdistan, dated archeologically and by radiocarbon from the 158 HUT period around 5400 BCE (Fig. S1; Table S2; Nieuwenhuyse et al., 2016; 159 Nieuwenhuyse, 2018; Odaka et al., 2019). In total, the Near-Eastern archeointensity database 160 contains 57 values (Table S1). Uniformly distributed age uncertainties were considered for all 161 these data.

\section{2.b Outline of the transdimensional Bayesian method AH-RJMCMC}

The transdimensional Bayesian method AH-RJMCMC (Age Hyperparameter Reverse

166 regional geomagnetic field intensities. The detailed description of this approach is provided in 167 the 2018 publication, and only the main points are outlined below. An ensemble of piecewise 168 linear fits to the data are calculated, whose statistics converge to those of the posterior 169 probability distribution of the intensity variation given the dataset. Each member of the 170 ensemble comprises a series of linear segments whose number, which defines the complexity 171 of the underlying geomagnetic variations, is determined by the data themselves according to 
172 their dating and experimental uncertainties. AH (from AH-RJMCMC) means that the ages of 173 the data are themselves introduced into the model vector, which makes it possible to take into 174 account, in addition to the uncertainties on dating, possible temporal relationships existing 175 between certain data or certain datasets (Livermore et al., 2018; Gallet et al., 2020; Shaar et 176 al., 2020). This is a crucial element when analyzing the Near-Eastern data obtained from 177 multi-layered archeological sites. The output is dependent on the prior information adopted, 178 which includes a maximum number of linear segments, set here to 150 , large compared to the 1792000 years of the time interval considered ( 7000-5000 BCE), and minimum and maximum 180 values of the intensity of each interior vertex, set respectively to $20 \mu \mathrm{T}$ and $70 \mu \mathrm{T}$. An ensemble size of 200 million individual models, large enough to ensure convergence, is computed following a perturbation scheme, using a Monte Carlo Markov chain algorithm, 183 applied to the number of segments (with the addition or removal of a segment), the age and 184 intensity of the knots and the data ages. Note that no form of weighting between the data, 185 other than that introduced by their experimental and age uncertainties, was implemented for 186 these calculations.

187 The AH-RJMCMC algorithm provides a probability density distribution as a function of 188 time for geomagnetic field intensity, for which the average, median, mode and $95 \%$ credible 189 interval are determined. Although each individual model is piecewise linear, these diagnostics 190 over the whole ensemble are smooth curves. By marginalization, it is also possible to 191 determine the posterior probability distributions of the age and intensity of each datum, which 192 indicate their most probable values given the characteristics (number, temporal distribution 193 and uncertainties) of the dataset available during the age interval studied. Finally, it should be 194 noted that an important advantage of this method lies in the absence of an ad-hoc 195 regularization parameter and that it therefore allows variations with very different timescales 196 to be determined, if these are actually required by the data. Such a characteristic is 
197 particularly valuable for identifying rapid variations as seems to be the case in the 6th millennium BCE (Livermore et al., 2018; 2021; Yutsis-Akimova et al., 2018a,b).

\section{Modeling results}

\section{3.a Balkans (6th millennium BCE)}

The evolution of the geomagnetic field intensity obtained in the Balkans from the AHRJMCMC method is shown in Fig. 1a (Table S3; note that all data were transferred to the latitude of Sofia, Bulgaria). The median values of the intensity probability distribution are reported because this indicator is less sensitive than the average to the extreme values given (2014) using the Bayesian method developed by Lanos (2004) (pale-orange area in Fig. 1a), is marked by two intensity peaks of $\sim 200$ years duration, the most recent with a maximum around $5555 \mathrm{BCE}$, the oldest with its maximum around $5805 \mathrm{BCE}$. The latter is still rather poorly defined due to the small number of data (two) available for the first two centuries of

211 the 6th millennium BC; this translates into a large credible interval during this period. The 212 minimum of geomagnetic intensities during the 6th millennium occurs around $5400 \mathrm{BCE}$ and 213 is followed by a steady increase in intensities until the end of the millennium. millennium BCE (Fig. 1a), suggesting rapid fluctuations. This is attested by the calculation of 216 the variation rates (time derivative of the median intensity curve) shown in Fig. 1b (Table S3). 217 The two intensity peaks mentioned above are associated with maximum rates of change of the 218 order of $\sim 0.25-0.27 \mu \mathrm{T} / \mathrm{year}$. This value is significantly higher than that mentioned by 219 Kostadinova-Avramova et al. (2019), with a value of $\sim 0.11 \mu \mathrm{T} /$ year estimated for the peak centered around $5550 \mathrm{BCE}$. This difference is related to both the different databases and 
221 characteristics of the two methods used for the calculation of geomagnetic evolutions (Lanos, 222 2004; Livermore et al., 2018), in particular with the absence of any regularization applied in 223 the AH-RJMCMC method. The data reported in Kovacheva et al (2014) were obtained over many years, and they

225 do not all share the same experimental criteria. In order to assess at first order the sensitivity 226 of the evolution described above to these experimental differences, the Balkan database was 227 subdivided according to whether or not the intensity determinations included pTRM checks 228 (Thellier and Thellier, 1959). When pTRM checks were carried out at least partially, i.e. for 229 the most recent data, the experimental uncertainties of the intensity values were kept as 230 published (circles in Figs. 1a and S2a,c). Conversely, when the data were not constrained by 231 pTRM checks, their experimental uncertainties have been arbitrarily set to $5 \mu \mathrm{T}$ when the 232 published ones are below this threshold (otherwise they have been kept as published; blue 233 triangles in Figs. 1a and S2a,c; Table S1). In addition, we roughly and arbitrarily considered 234 that the temporal uncertainties of the data could not be less than \pm 50 years. The variations in 235 geomagnetic field intensity calculated from this modified dataset are shown in Fig. S2a 236 (Table S3). These are very close to those exhibited in Fig. 1a, with two intensity peaks still 237 well expressed. This is also the case when the minimum temporal uncertainty is set to \pm 75 238 years (Fig. S2c; Tables S1, S3). The maximum rates of change associated with the intensity 239 peaks also remain of the order of 0.17-0.25 $\mu \mathrm{T} / \mathrm{year}$ (Fig. S2b,d). The variations shown in Fig. 240 1a thus appear reasonably robust, even if the acquisition of new archeointensity data appears 241 to be still necessary, especially for the beginning of the 6th millennium BCE. 

typo-morphology of ceramics and by some radiocarbon data (Bernbeck and Nieuwenhuyse, 2013; Molist et al., 2013; Yutsis-Akimova et al., 2018a,b; Nieuwenhuyse, 2018a, Gómez-

247 Bach et al., 2018a and references therein). Here we adopt a cautious approach that emphasizes 248 the link of the groups of potsherds with archeological periods (such as Middle Halaf or Late 249 Halaf) and the fact that these fragment groups are all associated with well-recognized 250 archeological levels, with time order relationships between them in the different sites. On the 251 other hand, the constraints provided by the relative thicknesses of the stratigraphic layers 252 identified at Yarim Tepe II and I (see in Yutsis-Akimova et al., 2018a,b) are ignored because 253 these thicknesses vary laterally on the surface of the sites and so certain stratigraphic layers 254 may have been partially or completely leveled, which casts some doubt on their relevance. 255 Neglecting the relative thicknesses of the layers also avoids the need for a priori knowledge 256 on the evolution of accumulation rates across these sequences. Hence, for these two sites, 257 temporal relationships were only considered between the different archeological levels 258 defined by the archeologists. This means that any subset of archeointensity data within a level 259 is not ordered in time (regardless of their stratigraphic position), but nevertheless must have 260 an age, which is (respectively) greater or younger than those data in levels immediately above 261 or below. This approach also places much less emphasis on individual dating of data (a 262 radiocarbon-dated bone fragment may have moved within a stratigraphic sequence; e.g. 263 Yutsis-Akimova et al., 2018b). It is thus very different from that previously used by Yutsis264 Akimova et al. (2018a,b).

The archeological periodization used and the associated dates are essentially those 266 given by Molist et al (2013) (see more discussion in Bernbeck and Nieuwenhuyse, 2013) and 267 Nieuwenhuyse (2018a). For the cultural phases preceding the Halaf (Initial Pottery Neolithic, 268 Early Pottery Neolithic, pre Halaf, proto-Halaf), the dating of the archeological transitions 
269 relies primarily on the large set of radiocarbon dates obtained at Tell Sabi Abyad, Syria (van 270 der Plicht et al., 2011, Nieuwenhuyse, 2018a). We arbitrarily assigned reasonable age 271 uncertainties of \pm 25 years to the dates of these transitions. This yields the Initial to Early

272 Pottery Neolithic transition dated to $6675 \pm 25$ BCE (base of level A9 at Tell Sabi Abyad dated 273 between 6675 and 6620 BCE; Nieuwenhuyse, 2018a), the Early Pottery Neolithic - pre Halaf 274 transition dated at $6635 \pm 25 \mathrm{BCE}$ (base of level A1 dated between 6335 and $6225 \mathrm{BCE}$; 275 Nieuwenhuyse, 2018a) and the pre Halaf - proto-Halaf transition dated at $6015 \pm 25$ BCE (this 276 transition occurring within level B3 dated between 6040 and 5995 BCE; Nieuwenhuyse, 277 2018a). Note that, following Nieuwenhuyse et al. (2010), the beginning of the Initial Pottery 278 Neolithic was placed at 7000 BCE without making a rough estimate (unnecessary for our 279 purpose) of the uncertainties on this date. The same age uncertainties of \pm 25 years were also 280 optimistically assigned to the proto-Halaf - Early Halaf, Early - Middle Halaf and Middle 281 Late Halaf transitions (5900 \pm 25 BCE, 5750 \pm 25 BCE, 5575 \pm 25 BCE, respectively; Bernbeck 282 and Nieuwensuyse, 2013; Molist et al., 2013; Gómez-Bach et al., 2018; Gómez-Bach and 283 Cruells, 2018). The case of the Late Halaf to HUT transition is different because the HUT 284 phase remains archeologically very poorly constrained (Campbell, 2007; Campbell and 285 Fletcher, 2010; see further discussion in Nieuwenhuyse et al., 2016 and Gómez-Bach et al., 286 2016). For this reason, its age uncertainties were arbitrarily increased to \pm 75 years, with a date 287 at $5350 \pm 75$ BCE. The time interval allowed for this transition (5425 - $5275 \mathrm{BCE})$ appears 288 reasonable in light of current archeological uncertainties (Campbell, 2007; O. Nieuwenhuyse, 289 personal communication). The end of HUT is just as loosely established. According to

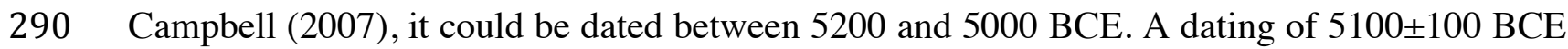
291 is thus roughly considered. More generally, the overall chronological framework above, 292 which is necessarily based on some approximations and arbitrary choices (some of which are 
293 probably rather optimistic), seems to us to reasonably reflect the current state of knowledge, 294 although other interpretations are possible.

The following age intervals were thus considered for the AH-RJMCMC calculations,

296 with the overlaps between them being treated by the algorithm through the time order 297 relationships:

+ Initial Pottery Neolithic (ceramic phase I at Tell Halula): 7000 - 6650 BCE

+ Early Pottery Neolithic (ceramic phase II at Tell Halula): 6700 - 6310 BCE

+ Pre Halaf (ceramic phase III at Tell Halula): 6360 - 5990 BCE

+ Proto-Halaf (ceramic phase IV at Tell Halula): 6040 - 5875 BCE

+ Early Halaf (ceramic phase V at Tell Halula): 5925 - 5725 BCE

+ Middle Halaf (ceramic phase VI at Tell Halula): 5775 - 5550 BCE

304

+ Late Halaf (ceramic phase VII at Tell Halula): 5600 - 5275 BCE

+ HUT: 5425 - 5000 BCE

The data obtained at Yarim Tepe I are archeologically dated to the archaic Hassuna

and standard Hassuna periods, which thus involves a different terminology from that with the pre-Halaf, proto-Halaf and Halaf. Here we use a correlation scheme close to that proposed by

BCE without approximation made for the dating of the transition between the archaic and

standard Hassuna, and with the possibility that part of the standard Hassuna defined in Iraq overlaps with part of the Early Halaf defined further east (Yutsis-Akimova et al., 2018b; O. Nieuwenhuyse, personal communication). 
316 individual data according to their prior values, it is the posterior values (median ages and 317 intensities with the $95 \%$ credible interval) that are exhibited (Fig. S3 shows the data using 318 both their prior and posterior age values). This figure underlines that despite the long duration 319 of the age intervals considered for most of the data, the time-order relationships existing 320 between them lead to a coherent evolution of the geomagnetic intensities during the 7 th and 321 6th millennia BCE. This evolution is marked by a steady decreasing trend during the 7th 322 millennium, until $\sim 6200 \mathrm{BCE}$, while it is more complex around the middle of the 6th 323 millennium BCE, with a sharp minimum around $35 \mu \mathrm{T}$ leading to an intensity peak between $324 \sim 5600 \mathrm{BCE}$ and $\sim 5400 \mathrm{BCE}$ (maximum around $5530 \mathrm{BCE}$ ). The maximum rates of change 325 associated with this intensity peak are of $\sim 0.37 \mu \mathrm{T} /$ year (Fig. 2b; Table S3), slightly higher 326 than those determined for the Balkans. It should be noted that the $95 \%$ credible interval 327 during these periods remains wide, especially during the first half of the 7th millennium BCE 328 where data are still scarce (note that these are the oldest archeointensity data obtained so far 329 from ceramics). On the other hand, the period between $6200 \mathrm{BCE}$ and $5600 \mathrm{BCE}$, i.e. 330 between the end of the pre-Halaf and the Middle Halaf (with data obtained at Tell Halula, 331 Yarim Tepe II and Yarim Tepe I), seems to be characterized by the lack of significant 332 intensity variations, as well as during the last three centuries of the 6th millennium BCE, the 333 latter period being also marked by the lowest geomagnetic intensities over the entire 2000334 year interval analyzed. It is worth pointing out that similar inferences are obtained if the time 335 order relationship considered for the Yarim Tepe II and Yarim Tepe I data is not between the 336 nine and eight different archeological levels as before, but between respectively 14 and 7 337 successive non-overlapping stratigraphic layers (still regardless of their thicknesses; Fig. 338 S4a,b; Tables S1, S3). In this case, the data obtained in overlapping stratigraphic layers are 339 grouped in a same layer without time order (see Table 1 in Yutsis-Akimova et al., 2018a,b). 
As previously mentioned, the Late Neolithic archeological chronologies established in

341 different areas of Mesopotamia are difficult to synchronize with each other. For this reason,

342 we tested at first order the robustness of the curves shown in Fig. 2 by ignoring in a second

343 calculation the age and stratigraphic position of the Middle - Late Halaf and Late Halaf -

344 HUT transitions inside the Yarim Tepe II sequence. This amounts to considering that the

345 Yarim Tepe II data lie in a single large time interval between 5800 and 5000 BCE, which

346 therefore puts fewer constraints on correlation with data obtained further west, in Tell Halula

347 and Tell Masaikh (Table S1). The AH-RJMCMC results presented in Fig. S4c,d show the

348 same intensity evolution as in Fig. 2, although the amplitude of the variations is significantly

349 smaller around 5600-5400 BCE (Table S3). The rates of change associated with the peak 350 around $5500 \mathrm{BCE}$ are also lower (maximum of $\sim 0.17 \mu \mathrm{T} / \mathrm{year}$ ), highlighting the sensitivity of 351 this parameter to archeological determinations or assumptions.

4. A master Upper Mesopotamian archeointensity variation curve for the Pottery

\section{Neolithic}

355 The regional geomagnetic field intensity models obtained for the Balkans and Upper Mesopotamia show the same peak in intensity around the middle of the 6th millennium BCE, with similar rates of change. This underlines, at least for this period, a good homogeneity of 358 the secular variation of intensity in a large area between Bulgaria and the Near East (with a 359 distance of $\sim 1600 \mathrm{~km}$ between Sofia, Bulgaria, and the archeological site of Tell Halaf in 360 Syria). However, two main differences are observed. The first concerns the intensity peak observed around $5800 \mathrm{BCE}$ in the Balkans but not in the Near East. This age corresponds to 362 the Early Halaf (e.g. Molist et al, 2013; Bernbeck and Nieuwenhuyse, 2013), for which a 363 single archeointensity result was obtained at Tell Halula (Gallet et al., 2015), while there is a 364 question as to whether the recent part of the so-called standard Hassuna period (with data 
obtained at Yarim Tepe I) could extend to the beginning of the Early Halaf (Yutsis-Akimova et al., 2018b; O. Nieuwenhuyse, personal communication). The second difference is in the

367 behavior of the geomagnetic field at the end of the 6th millennium BCE, marked by an 368 increase in intensities in the Balkans while they are fairly constant in the Near East. It is 369 worth remarking that these two differences occur when comparing the median posterior 370 models, it does not mean that the datasets are themselves mutually incompatible. It remains 371 important to test whether the data obtained independently in the Balkans and in the Near East 372 can produce a coherent evolution of geomagnetic intensities throughout the 6th millennium 373 BCE, integrating the characteristics mentioned above. In other words, this amounts to 374 determining what would be the influence, or implication, of the data available in the Balkans 375 on the pattern of intensity variations in Upper Mesopotamia, assuming that the two regions shared the same secular variation in intensity during this time interval. Such a reconciliation between these two datasets might be achieved by virtue of the uncertainty in both intensity and ages, allowing the posterior distribution to effectively shift the data in both intensity and 379 time (within their given prior distributions) to a mutually favorable configuration, in which all 380 data are compatible with a single intensity variation curve.

A master intensity variation curve was determined for Upper Mesopotamia by transferring all Balkan data to the latitude of Tell Halaf (the Near-Eastern data used for Fig. 383 2a remaining unchanged). However, given the rather large distance between Bulgaria and 384 northern Syria, this transfer carried out using the hypothesis of a simple geocentric axial 385 dipole raises uncertainties. These are illustrated by a simple calculation: the intensity 386 determined in Sofia from the most recent IGRF (Alken et al., 2021) is $47.9 \mu \mathrm{T}$; its transfer to 387 the latitude of Tell Halaf using the same hypothesis gives $44.7 \mu \mathrm{T}$, to be compared to $47.6 \mu \mathrm{T}$ 388 determined at Tell Halaf from the IGRF. The difference of $\sim 3 \mu \mathrm{T}$ corresponds to $\sim 7 \%$ of the 389 "true" value, which is far from negligible. In order to take into account this discrepancy at 
390 first order, without of course knowing its evolution over the past millennia, we arbitrarily 391 considered that the experimental errors on the Balkan intensity values transferred to the Near 392 East could not be less than $3 \mu \mathrm{T}$ (same approach as before). On the other hand, we note that 393 the intensity variation curve derived from the Balkan data transferred to the latitude of Tell 394 Halaf might indicate a shift in intensity around 5700-5400 BCE with respect to the Near395 Eastern curve (Fig. S3). If significant, the origin of this offset would remain unclear. A 396 possible bias due to the effect of cooling rate on thermoremanent magnetization acquisition 397 (see discussion for example in Genevey et al., 2008) could be suggested for the Balkan data, 398 however, this effect was considered essentially negligible for the entire dataset (Fanjat et al., 399 2013; Kovacheva et al., 2014; Kostadinova-Avramova et al., 2019). Furthermore, to account very roughly for the fact that the signature of the non-dipole 401 component of the geomagnetic field may have drifted slightly from east to west, or from west 402 to east between the Balkans and the Near East during the 6th millennium BCE, we arbitrarily 403 considered that the dating of the Balkan data could not be more accurate than \pm 75 years 404 (Table S1). This appears all the more justified since the Balkan curve transferred to the 405 latitude of Tell Halaf also seems to present around 5700-5400 BCE a time delay of $~ 50$ years 406 with respect to the Near-Eastern curve (Fig. S3). However, given the data currently available 407 and the error bars of the models, it seems premature to consider a phenomenon of eastward 408 drift of the secular variation at this period and to shift the age of the Balkan data as a whole 409 by $\sim 50$ years. By increasing the age uncertainties of the Balkan data, this offset, if significant, 410 should be accounted for in the AH-RJMCMC calculations. 412 posterior distribution from only the Balkan dataset and use it as a prior to calculate the 413 posterior distribution from the Upper Mesopotamian dataset. Alternatively, we can combine 414 the two datasets and discover the posterior in a single calculation from this super-set. We 
adopt the latter strategy as it is computationally much simpler and accordingly a database of

416108 data was compiled to trace the geomagnetic field intensity evolution in Upper

417 Mesopotamia (Table S1). Below, we focus only on the 6th millennium BCE.

This master curve is shown in Fig. 3a (Table S3). As in Fig. 2a, the data are reported using their median posterior age and intensity values and the associated $95 \%$ credible interval

420 (see details of the different symbols in the figure caption). The median intensity curve (in blue) shows a regular evolution on the multi-century time scale, which is however associated with a rather wide $95 \%$ credible interval (shaded area). This evolution now integrates the intensity peak around $5800 \mathrm{BCE}$ (maximum at $5810 \mathrm{BCE}$ ) seen from the Balkan data, 424 whereas the plateau previously mentioned during the Middle Halaf is strongly attenuated (Fig. 425 2a). Unsurprisingly an intensity peak is clearly observed around 5550 BCE (date of the maximum). A major change concerns the end of the 6th millennium BCE where the medians

427 of the posterior age values obtained for the HUT data are all concentrated around $5300 \mathrm{BCE}$, 428 allowing the increase in geomagnetic intensities observed from the Balkan data.

430 illustrated by the posterior probability distributions of median ages for some archeointensity 431 data (Fig. 4; data indicated by open symbols in Fig. 3a). For the only result of the Early Halaf 432 obtained at Tell Halula (SY91, Fig. 4a), it can be seen that the posterior age is mainly 433 concentrated at both sides of the prior age interval, which leaves some room (in terms of 434 probability) for a peak of intensity lasting about a century towards the middle of this 435 archeological period. Moreover, two HUT results obtained at Tell Masaikh (SY37, Fig. 4b) and Yarim Tepe II (YT01, Fig. 4c) show that the posterior probability distribution of their age

437 values is strongly concentrated in the older part of their prior age interval, suggesting that 438 only a small part of the HUT (i.e. the oldest part) was sampled in the two sites (and see 
439 below). Such a possibility had already been raised by Yutsis-Akimova et al (2018a), but only

440 on a purely empirical basis.

441 With respect to the rates of intensity change, combining the Balkan and Near-East data

442 in the calculation yields maximum values of $\sim 0.22 \mu \mathrm{T} / \mathrm{year}$ for the peak around $5800 \mathrm{BCE}$,

443 similar to those previously obtained from the Balkan data alone (Fig. 1b). Conversely, the

444 maximum rates of change are higher $(\sim 0.52 \mu \mathrm{T} / \mathrm{year})$ for the peak around $5550 \mathrm{BCE}$, albeit

445 with large uncertainties, than when considering the Balkan and Near East data separately. At

446 this stage, it is unclear whether these values are relevant from a geomagnetic point of view

447 given the approximations that have been made to combine together the Near East and Balkan

448 data. In any case, the variation rates associated with the peak around 5550 BCE appear to be

449 at least $\sim 0.2 \mu \mathrm{T} /$ year, about twice the maximum rate observed in the modern geomagnetic

450 field (Alken et al., 2021).

\section{Concluding remarks on the archeological inferences}

Although Near-Eastern archeointensity datasets are still limited for the Late Neolithic,

454 the use of the AH-RJMCMC method is already shedding light on archeological issues, whose

455 resolution remains largely in the realm of archeology. The new insights can be summarized as 456 follows:

- The Upper Mesopotamian data available for the Early Halaf are currently too sparse to

458 reliably trace the geomagnetic field intensity variations during this period. Confirmation in

459 Upper Mesopotamia of the intensity peak around 5800 BCE, still observed only from the 460 Balkan data, would provide a crucial chronological marker, all the more important as recent 461 excavations in Iraqi Kurdistan show that, according to the ceramic typology, the Early Halaf 462 time interval is not clearly attested in this region (Nieuwenhuyse, 2018b and personal 

extend into the Early Halaf (Yutsis-Akimova et al., 2018b; see also discussion in Cruells and

465 Nieuwenhuyse, 2004), this overlap should not extend beyond the beginning of the Early Halaf 466 defined further east.

467 - According to the Balkan results, all the archeointensity data obtained so far in Upper 468 Mesopotamia for the Halaf-Ubaid Transitional could be dated to the beginning of this 469 archeological phase, whose transition with the Late Halaf, defined from subtle changes in the 470 typology and decoration of ceramics, is currently poorly circumscribed (e.g. Campbell, 2007; 471 Nieuwenhuyse, 2018b and references therein). From the archeomagnetic point of view, the 472 alternatives are the following: either the sampled HUT actually corresponds to the Late Halaf, 473 or only the very beginning of the HUT is represented at Tell Masaikh and Yarim Tepe II, or 474 the HUT was shorter than expected and does not span the last two centuries of the 6th 475 millennium BCE (occurring between 5400 and 5200 BCE). It should also be noted that, at 476 this stage, the available data are insufficient to test whether the HUT could have had a 477 different duration between the east and west of Upper Mesopotamia. These various options 478 echo the large archeological uncertainties still existing on the HUT phase.

479 - A close examination of Figs. 2 and S4 indicates that part of the Late Halaf present at Tell 480 Halula, at 5500-5450 BCE (see blue dots intercalated between red dots), might be absent at 481 Yarim Tepe II, suggesting a short hiatus in the latter sequence, while the end of the Late Halaf 482 may be absent in Tell Halula. At this stage, such a possibility is statistically far from proven, 483 but it might be further analyzed in light of the comparative changes in ceramic typology 484 between the two sequences. In addition, it seems conceivable that the very thin thickness $(\sim 30$ $485 \mathrm{~cm}$ ) of archeological level VII at Yarim Tepe II, compared to the thicknesses of the other 486 levels, is the result of low accumulation rates, perhaps a short hiatus, around the Middle to 487 Late Halaf transition, which an age model constructed primarily from the thicknesses of the 
archeological levels and layers, in contrast to our AHRJMCMC age model, tends to minimize due to lack of sufficient dating constraints (see Figs. 7 and 8 in Yutsis-Akimova et al., 2018a). In conclusion, the rapid variations in geomagnetic field intensities during the 6th

491 millennium BCE as determined by the AH-RJMCMC method offer promising constraints for 492 the correlation of archeological sequences established from widely distributed settlements in 493 the Near East. In particular, these variations could help to synchronize the pottery horizons 494 and/or archeological layers discovered in Upper Mesopotamia (Turkey, Syria, Iraq), the 495 Levant and in the Zagros (Iran) region (e.g. Akkermans and Schwartz, 2003; Nieuwenhuyse 496 et al., 2013; Gómez-Bach et al., 2018a).

\section{Acknowledgements}

499 This study is dedicated to the memory of Olivier Nieuwenhuyse. YG is very grateful to him 500 for the very enriching discussions and helpful advise, unfortunately interrupted far too early. 501 He also provided the pottery fragments from Tell Begum. YG thanks Miquel Molist and 502 Shahrmardan Amirov who made possible archeointensity data acquisition in Tell Halula, 503 Yarim Tepe II and I and for stimulating discussions. Special thanks are due to Stasya Yutsis504 Akimova who analyzed the potsherds from Yarim Tepe I and II in the framework of her PhD 505 thesis. Additional thanks are due to Anna Gómez-Bach for her comments on a preliminary 506 version of the manuscript, and to T. Odaka for archeological information on Tell Begum. We 507 finally thank Stuart Campbell and Javier Pavón-Carrasco for reviewing the manuscript and 508 providing helpful comments. This is IPGP contribution no. 4222.

\section{References}


511 Akkermans, P.M.M.G., Schwartz, G.M., 2003. The Archaeology of Syria. From Complex

512 Hunter-gatherers to Early Urban Societies (ca. 16,000-300 BC). Cambridge World 513 Archaeology, Cambridge University Press, New York, pp. 467.

514 Alken, P., Thébault, E., Beggan, C.D., Amit, H., Aubert, J., Baerenzung, J., Bondar, T.N., 515 Brown, W., Califf, S., Chambodut, A., Chulliat, A., Cox, G., Finlay, C.C., Fournier, A., 516 Gillet, N., Grayver, A., Hammer, M.D., Holschneider, M., Huder, L., Hulot, G., Jager, T., 517 Kloss, C., Korte, M., Kuang, W., Kuvshinov, A., Langlais, B., Léger, J.M., Lesur, V., 518 Livermore, P.W., Lowes, F.J., Macmillan, S., Magnes, W., Mandea, M., Marsal, S., Matzka, 519 J., Metman, M.C., Minami, T., Morschhauser, A., Mound, J.E., Nair, M., Nakano, S., Olsen, 520 N., Pavón-Carrasco, F.J., Petrov, V.G., Ropp, G., Rother, M., Sabaka, T.J., Sanchez, S., 521 Saturnino, D., Schnepf, N.R., Shen, X., Stolle, C., Tangborn, A., Toffner-Clausen, L., Toh, 522 H., Torta, J.M., Varner, J., Vervelidou, F., Vigneron, P., Wardinski, I., Wicht, J., Woods, A., 523 Yang, Y., Zeren, Z., Zhou, B., 2021. International Geomagnetic Reference Field: the 524 thirteenth generation. Earth Planets Space 73, 49.

525 Amirov, S., 1994. The morphology of Halafian culture ceramics based on the material 526 collected from the settlement Yarim Tepe II (In Russian). Doctoral thesis, Moscow., pp. 144.

527 Amirov, S., 2018. The morphology of Halafian painted pottery from Yarim Tepe II, and the 528 process of Ubaidian acculturation. In: Workshop on Late Neolithic ceramics in Ancient 529 Mesopotamia in context, A. Gómez-Bach, J. Becker and M. Molist (eds.), Monografies del 530 MAC 1, Barcelona, 13-21.

531 Bader, N., 1989. Earliest cultivators in Northern Mesopotamia. The Investigations of Soviet 532 Archaeological Expedition in Iraq at Settlements Tell Magzaliya, Tell Sotto, and Kül Tepe. 533 Moscow, Nauka.

534 Bashilov, V.A., Bolshakov, O.G., Kouza, A.V., 1980. The earliest strata of Yarim Tepe I. 535 Sumer 36, 43-64. 
536 Bernbeck, J., Nieuwenhuyse, O.P., 2013. Established paradigms, current disputes and 537 emerging themes: the state of research on the Late Neolithic in Upper Mesopotamia. In: 538 Interpreting the Late Neolithic of Upper Mesopotamia. Publications on Archaeology of the 539 Leiden Museum of Archaeology (PALMA), Brepols pub. (Turnhout, Belgium), pp. 17-37.

540 Campbell, S., 2007. Rethinking Halaf chronology. Paléorient 33, 103-136.

541 Campbell, S., Fletcher, A., 2010. Questioning the Halaf-Ubaid transition. In: Carter, R.A., 542 Philip, G. (Eds.), Beyond the Ubaid: Transformation and Integration in the Late Prehistoric 543 Societies of the Middle East, SAOC: Studies in Ancient Oriental Civilization 63. The Oriental 544 Institute of the University of Chicago, pp. 69-83.

545 Cruells, W., Nieuwenhuyse, O., 2004. The Proto-Halaf period in Syria. New sites, new data. 546 Paléorient 30, 47-68.

547 Davidson, T.E; McKerrell, H., 1980. The Neutron Activation Analysis of Halaf and Ubaid 548 Pottery from Tell Arpachiyah and Tepe Gawra, Iraq 42, 155-167.

549 Davidson, T.E., 1981. Pottery Manufacture and Trade at the Prehistoric Site of Tell Aqab, 550 Syria. Journal of Field Archaeology 8, 65-77

551 Fanjat, G., Aidona, E., Kondopoulou, D., Camps, P., Rathossic C., Poidras T., 2013. 552 Archeointensities in Greece during the Neolithic period: New insights into material selection 553 and secular variation curve. Phys. Earth Planet. Inter. 215, 29-42.

554 Forest, J.-D., 2013. The birth of a new culture: at the origin of the Halaf. In: Interpreting the 555 Late Neolithic of Upper Mesopotamia. Publications on Archaeology of the Leiden Museum 556 of Archaeology (PALMA), Brepols pub. (Turnhout, Belgium), pp. 101-105.

557 Frangipane, M., 2013. Societies without boundaries: interpreting Late Neolithic patterns of 558 wide interaction and sharing of cultural traits. The case of the Halaf communities. In: 
559 Interpreting the Late Neolithic of Upper Mesopotamia. Publications on Archaeology of the

560 Leiden Museum of Archaeology (PALMA), Brepols pub. (Turnhout, Belgium), pp. 89-99.

561 Gallet, Y., Le Goff, M., 2006. High-temperature archeointensity measurements from

562 Mesopotamia. Earth Planet. Sci. Lett. 241, 159-173.

563 Gallet, Y., Genevey, A., Le Goff, M., Warmé, N., Gran-Aymerich, J., Lefèvre, A., 2009. On

564 the use of archeology in geomagnetism, and vice-versa: Recent developments in

565 archeomagnetism, C. R. Physique 10, 630-648.

566 Gallet, Y., Molist, M., Genevey, A., Clop Garcia, X., Thébault, E. Gómez Bach, A., Le Goff,

567 M. Robert, B., Nachasova, I., 2015. New Late Neolithic (c. 7000-5000 BC) archeointensity

568 data from Syria. Reconstructing 9000 years of archeomagnetic field intensity variations in the

569 Middle East. Phys. Earth Planet. Inter. 238, 89-103.

570 Gallet, Y., Fortin, M., Fournier, A., Le Goff, M., Livermore, P., 2020. Analysis of 571 geomagnetic field intensity variations in Mesopotamia during the third millennium BC with 572 archeological implications. Earth Planet. Sci. Lett. 537, 116183.

573 Genevey, A., Gallet, Y., Constable, C.G., Korte, M., Hulot, G., 2008. ArcheoInt: an up574 graded compilation of geomagnetic field intensity data for the past ten millennia and its 575 application to the recovery of the past dipole moment. Geochem. Geophys. Geosyst. 9, $576 \quad$ Q04038

577 Genevey, A., Gallet, Y., Thébault, E., Livermore P.W., Fournier, A., Jesset, S., Lefèvre, A., 578 Mahé-Hourlier, N., Marot, E., Regnard S., 2021. Archeomagnetic intensity investigations of 579 French Medieval ceramic workshops: Contribution to regional field modeling and 580 archeointensity-based dating. Phys. Earth Planet. Inter. 318, 106750.

581 Gómez-Bach, A., Cruells, W., Molist, M., 2016. Sharing spheres of interaction in the $6^{\text {th }}$ 582 millennium cal. BC: Halaf communities and beyond. Paléorient 42, 117-133. 
583 Gómez-Bach, A., Cruells, W., 2018. Time and technological transfer in proto-Halaf and Halaf 584 sequences at Chagar Bazar (Khabur valley, Syria). In: Workshop on Late Neolithic ceramics 585 in Ancient Mesopotamia in context, A. Gómez-Bach, J. Becker and M. Molist (eds.), 586 Monografies del MAC 1, Barcelona, 67-82.

587 Gómez-Bach, A., Becker, J., Molist, M. (eds.), 2018a. Workshop on Late Neolithic ceramics 588 in Ancient Mesopotamia in context, Monografies del MAC 1, Barcelona, 45-57.

589 Gómez-Bach, A., Cruells, W., Molist, 2018b. Halaf phenomena: surplus, homeland and 590 identity in Upper Mesopotamia (6.200-5.300 cal BC). In: Herusgeber, H.M.; Detlef, G.; 591 Risch, R., Surplus without the State. Political Forms in Prehistory. 10. Mitteldeutscher 592 Archäologentag, vom 19 bis 21 Oktober 2017 in Hale (Saale), Tagungen des Landesmuseums 593 für Vorgeschichte Halle, Band 18, 147-166.

594 Gómez-Bach, A., Clop, X., Molist, M., 2018c. Red ware: characterizing a pottery production 595 at Tell Halula at mid-sixth millennium cal BC. In: Workshop on Late Neolithic ceramics in 596 Ancient Mesopotamia in context, A. Gómez-Bach, J. Becker and M. Molist (eds.), 597 Monografies del MAC 1, Barcelona, 141-147.

598 Hervé, G., Lanos, P., 2017. Improvements in Archaeomagnetic Dating in Western Europe 599 from the Late Bronze to the Late Iron Ages: An Alternative to the Problem of the Hallstattian 600 Radiocarbon Plateau: Improvements in archaeomagnetic dating in Western Europe. 601 Archaeometry 60 (4), 870-883.

602 Hole, F., 2013. Constrained innovation: Halafian ceramics. In: Interpreting the Late Neolithic 603 of Upper Mesopotamia. Publications on Archaeology of the Leiden Museum of Archaeology 604 (PALMA), Brepols pub. (Turnhout, Belgium), pp. 77-87.

605 Huot, J.-L., 2004. Une archéologie des peuples du Proche-Orient I. Des premiers villageois 606 aux peuples des cités-états. Editions Errance, Paris, pp. 249. 
607 Korte, M., Brown, M., Gunnarson, S.R., Nilsson, A., Panovska, S., Wardininski, I., Constable

608 C.G., 2019. Refining Holocene geochronologies using paleomagnetic records. Quaternay

609 Geochronology 50, 47-74.

610 Kostadinov-Avramova, M., Kovacheva, M., Boyadzhiev, 2014. Contribution of stratigraphic

611 constraints of Bulgarian prehistoric multilevel tells and a comparison with archaeomagnetic

612 observations. J. Archaeol. Sci. 43, 227-238.

613 Kostadinov-Avramova, M., Kovacheva, M., Boyadzhiev, Y., Hervé, G., 2019.

614 Archaeomagnetic knowledge of the Neolithic in Bulgaria with emphasis on intensity changes.

615 Geol. Soc. Lond., Spec. Publ. 497.

616 Kovacheva, M., Kostadinova-Avramova, M., Jordanova, N., Lanos, P., Boyadziev, Y., 2014.

617 Extended and revised archaeomagnetic database and secular variation curves from Bulgaria

618 for the last eight millennia. Phys. Earth Planet. Inter. 236, 79-94.

619 Lanos, P., 2004. Bayesian inference of calibration curves: application to archaeomagnetism,

620 in: Tools for Constructing Chronologies. Springer, pp. 43-82.

621 Le Goff, M., Gallet, Y., Genevey, A., Warmé, N., 2002. On archaeomagnetic secular

622 variation curves and archaeomagnetic dating. Phys. Earth Planet. Inter. 134, 203-211.

623 Le Goff, M., Gallet, Y., 2004. A new three-axis vibrating sample magnetometer for

624 continuous high-temperature magnetization measurements: applications to paleo- and archeo-

625 intensity determinations. Earth Planet. Sci. Lett. 229, 31-43.

626 Livermore, P.W., Fournier, A., Gallet, Y., Bodin, T., 2018. Transdimensional inference of

627 archeomagnetic intensity change. Geophys. J. Int. 215, 2008-2034.

628 Livermore, P.W., Gallet, Y., Fournier, A., 2021. Archeomagnetic intensity variations during 629 the era of geomagnetic spikes in the Levant. Phys. Earth Planet. Inter. 312, 106657. 
Merpert, N.J., Munchaev, R.M., 1987. The Earliest Levels at Yarim Tepe I and Yarim Tepe II in Northern Iraq. Iraq 49, 1-36.

Merpert, N.J., Munchaev, R.M. 1993a. Yarim Tepe I. In: Early stages in the evolution of

633 Mesopotamian civilizations: Soviet excavations in Northern Iraq, N. Yoffee and J. Clark 634 (Ed.), pp. 73-114. Tucson: University of Arizona Press.

635 Merpert, N.J., Munchaev, R.M. 1993b. Yarim Tepe II: The Halaf levels. In: Early stages in 636 the evolution of Mesopotamian civilizations: Soviet excavations in Northern Iraq, N. Yoffee 637 and J. Clark (Ed.), pp. 129-162. Tucson: University of Arizona Press.

638 Molist, M., Anfruns, J., Bofill, M., Borrell, F., Buxy, R., Clop, X., Cruells, W., Faura, J.M., 639 Ferrer, A., Gómez, A., Guerrero, E., Saca, M., Tornero, C., Vicente, O., 2013. Tell Halula 640 (Euphrates Valley, Syria): New approach to VII and VI millennia cal. B.C. in Northern 641 Levant framework. In: Interpreting the Late Neolithic of Upper Mesopotamia. Publications on 642 Archaeology of the Leiden Museum of Archaeology (PALMA), Brepols pub. (Turnhout, 643 Belgium), pp. 443-455.

644 Nieuwenhuyse, O., Akkermans, P., van der Plicht, J., 2010. Not so coarse, nor always plain 645 the earliest pottery of Syria. Antiquity 84, 71-85.

646 Nieuwenhuyse, O.P., Bernbeck, R., Akkermans, P.M.M.G., Rogasch, J., 2013. Interpreting 647 the Late Neolithic of Upper Mesopotamia. Publications on Archaeology of the Leiden 648 Museum of Archaeology (PALMA), Brepols pub. (Turnhout, Belgium), pp. 520.

649 Nieuwenhuyse, O., 2013. The social uses of decorated ceramics in Late Neolithic Upper 650 Mesopotamia. In: Interpreting the Late Neolithic of Upper Mesopotamia. Publications on 651 Archaeology of the Leiden Museum of Archaeology (PALMA), Brepols pub. (Turnhout, 652 Belgium), pp. 135-145. 
653 Nieuwenhuyse, O., Odaka, T., Kaneda, A., Mühl, S., Rasheed, K., Altaweel, M., 2016.

654 Revisiting Tell Begum: a prehistoric site in the Shahrizor plain, Iraqi Kurdistan. Iraq 78, 103655135.

656 Nieuwenhuyse, O. (Ed.), 2018a. Relentlessly plain: seventh millennium ceramics at Tell Sabi 657 Abyad, Syria. Oxbow Books, Oxford \& Philadelphia, 396 p.

658 Nieuwenhuyse, O., 2018b. In the Shahrizor. Reassessing the Halaf ceramic traditions of Iraqi 659 Kurdistan. In: Workshop on Late Neolithic ceramics in Ancient Mesopotamia in context, A. 660 Gómez-Bach, J. Becker and M. Molist (eds.), Monografies del MAC 1, Barcelona, 43-55.

661 Odaka, T., Nieuwenhuyse, O., Mühl, S., 2019. From the $7^{\text {th }}$ to the $6^{\text {th }}$ millennium BC in Iraqi 662 Kurdistan: a local ceramic horizon in the Shahrizor plain. Paléorient 45 (2), 67-83.

663 Pavón-Carrasco, F.J., Rodríguez-González, J., Osete, M. L., Torta, J. M., 2011. A MATLAB 664 tool for archaeomagnetic dating. J. Archaeol. Sci. 38, 408-419.

665 Principe, C., Gogichaishvili, A., Arrighi, S., Devidze, M., La Felice, S., Paolillo, A., 666 Giordano, D., Morales, J., 2018. Archaeomagnetic dating of Copper Age furnaces at Croce di 667 Papa village and relations on Vesuvius and Phlegraean Fields volcanic activity. J. Volcanol. 668 Geotherm. Res. 349, 217-229.

669 Robert, B., Blanc, C., Masetti-Rouault, M-G. 2008. Characterizing the Halaf-Ubaid 670 Transitional Period by studying ceramic from Tell Masaikh, Syria. Archaeological data and 671 archeometry investigations. In : Kühne H., Czichon R.M., Kreppner F.J. (ed.), Proceedings of 672 4th International Congress of the Archaeology of the Ancient Near East, Wiesbaden, 673 Harrassowitz, 225-234

674 Robert, B., 2010. Développement et disparition de la production céramique halafienne: 675 implications techniques et sociales à partir d'études de cas. PhD thesis Université Lumière 676 Lyon 2, pp. 899. 
677 Schnepp, E., Obenaus, M., Lanos, P., 2015. Posterior archaeomagnetic dating: An example

678 from the Early Medieval site Thunau am Kamp, Austria. J. Archaeol. Sci. Rep. 2, 688-698.

679 Shaar, R., Bechar, S., Finkelstein, I., Gallet, Y., Martin, M., Ebert, Y., Keinan, J., Gonen, L., 680 2020. Synchronizing geomagnetic field intensity records in the Levant between the 23rd and 681 15th centuries BCE: chronological and methodological implications. Geochem., Geophys., 682 Geosyst. 21, e2020GC009251.

683 Spataro, M., Fletcher, A., 2010. Centralisation or regional identity in the Halaf period? 684 Examining interactions within fine painted ware production. Paléorient, 36, 91-116.

685 Thellier, E., Thellier, O., 1959. Sur l'intensité du champ magnétique terrestre dans le passé 686 historique et géologique. Ann. Géophys. 15, 285-376.

687 Tsuneki A., Nieuwenhuyse O., Campbell, S. (eds.), 2017. The Emergence of Pottery in West 688 Asia. Oxford and Philadelphia: Oxbow Books. 192 pp.

689 Van der Plicht, J., Akkermans, P.M.M.G., Nieuwenhuyse, O., Kaneda, A., Russell, A. 2011. 690 Tell Sabi Abyad, Syria: radiocarbon chronology, cultural change, and the 8.2 ka event. 691 Radiocarbon 53, 229-243.

692 Yutsis-Akimova, S., Gallet, Y., Amirov, S., 2018a. Rapid geomagnetic field intensity 693 variations in the Near East during the $6^{\text {th }}$ millennium BC: New archeointensity data from 694 Halafian site Yarim Tepe II (Northern Iraq). Earth Planet. Sci. Lett. 482, 201-212.

695 Yutsis-Akimova, S., Gallet, Y., Petrova, N., Nowak, S., Le Goff, M., 2018b. Geomagnetic 696 field in the Near East at the beginning of the 6th millennium BC: Evidence for alternating 697 weak and strong intensity variations. Phys. Earth Planet. Inter. 282, 49-59. 
Figure captions

701

702 Fig. 1. (a) Evolution of the geomagnetic field intensity in the Balkans during the $6^{\text {th }}$ 703 millennium BCE estimated using the AH-RJMCMC method (Livermore et al., 2018). Blue 704 dots and triangles: data with and without pTRM checks from Kovacheva et al. (2014); red 705 dots: data from Kostadinova-Avramova et al. (2019); green dot: a result from Greece obtained 706 by Fanjat et al. (2013); see text for further description. The variation curve (median values) is 707 in blue and the light blue shaded area show its $95 \%$ credible interval. The average curve 708 calculated by Kovacheva et al. (2014) using the Lanos (2004) method is also shown (pale 709 orange). (b) Medians and credible interval of the intensity variation rates estimated for the 710 Balkans using the AH-RJMCMC method. All data are transferred to the latitude of Sofia $711\left(\lambda=42.70^{\circ} \mathrm{N}\right)$.

712 Fig. 2. Same as in Fig. 1 but for the Upper Mesopotamian region during the $7^{\text {th }}$ and $6^{\text {th }}$ 713 millennia BCE. Blue dots and triangles: data from Tell Halula and Tell Masaikh (Gallet et al., 714 2015); red dots and triangles: data from Yarim Tepe II (Yutsis-Akimova et al., 2018a) and 715 Yarim Tepe I (Yutsis-Akimova et al., 2018b). All data are transferred to the latitude of Tell 716 Halaf (Syria; $\lambda=36.82^{\circ} \mathrm{N}$ ). The archeological periodization is shown at the top of the figure.

717 The grey zones indicate the estimated uncertainties in the age of the archeological transitions 718 (see text).

719 Fig. 3. Same as in Figs. 1 and 2 but for a dataset combining the results available from the 720 Balkans and Upper Mesopotamia. See text for changes to the Balkan archeointensity data. 721 Same symbols and color code as before for the Upper Mesopotamian data, except for three 722 data shown by empty symbol that are further illustrated in Fig. 4 (dot circled in blue: SY91, 
723 Tell Halula; triangle with blue lines: SY37, Tell Masaikh; dot circled in red: YT01, Yarim

724 Tepe II). Grey dots, triangles and square: Data from the Balkans obtained by Kovacheva et al.

725 (2014), Kostadinova-Avramova et al. (2019) and Fanjat et al. (2013), respectively.

726 Fig. 4. Comparison between the joint posterior probability distributions of the median age and 727 intensity values (green bars) and the corresponding prior values (pale orange) for three 728 different archeointensity results. (a) Early Halaf-dated result obtained at Tell Halula (SY91; 729 Gallet et al., 2015); (b) HUT result from Tell Masaikh (SY37; Gallet et al., 2015); (c) HUT 730 result from Yarim Tepe II (YT01; Yutsis-Akimova et al., 2018a).

\section{Supplementary material}

733 Fig. S1. New archeointensity data from Tell Begum $\left(\lambda=35^{\circ} 17^{\prime} 5^{\prime} ’ \mathrm{~N}, \phi=45^{\circ} 53^{\prime} 05^{\prime \prime} \mathrm{E}\right)$ in Iraqi 734 Kurdistan obtained from HUT fine ware pottery. Archeological reference: Level IV, Lower 735 Trench (LT) sounding locus 4, BEG39-40. The data were obtained using the Triaxe protocol 736 (Le Goff and Gallet, 2004) and obey the same selection criteria as for instance in Gallet and 737 Le Goff (2006), Gallet et al. (2015; 2020), Yutsis-Akimova et al. (2018a,b). Each curve 738 shows the R'(Ti) data obtained for one specimen (Le Goff and Gallet, 2004), with a minimum 739 of two, but more often three specimens successfully analyzed per fragment. Five fragments 740 provided archeointensity results, although the presence of a secondary magnetization 741 component in these fragments required intensity determinations at relatively high 742 temperatures $\left(\mathrm{T}^{\prime}{ }^{\prime}>300^{\circ} \mathrm{C}\right.$; see details in Gallet and Le Goff, 2004 and other references 743 mentioned above).

744 Fig. S2. (a,c) Evolution of the geomagnetic field intensities in the Balkans during the $6^{\text {th }}$ 745 millennium BCE estimated using the AH-RJMCMC method (Livermore et al., 2018). Blue 746 dots and triangles: data with and without pTRM checks from Kovacheva et al. (2014); red 
747 dots: data from Kostadinova-Avramova et al. (2019); green dot: a result from Greece obtained

748 by Fanjat et al. (2013); see text for further description. The variation curve (median values) is

749 in blue and the shaded area show its $95 \%$ credible interval. For these computations, the data

750 that were not constrained by pTRM checks were modified so that their minimum

751 experimental errors cannot be less than $\pm 5.0 \mathrm{mT}$ (see text). In addition the dating accuracy of

752 all the data cannot be more accurate than \pm 50 years (a) and \pm 75 years (c). (b,d) Medians and

$75395 \%$ credible interval of the intensity variation rates estimated for the Balkans using the $\mathrm{AH}-$

754 RJMCMC method (with minimum age uncertainties of \pm 50 years (b) and \pm 75 years (d)). All

755 data are transferred to the latitude of Sofia $\left(\lambda=42.70^{\circ} \mathrm{N}\right)$.

756 Fig. S3. Evolution of the geomagnetic field intensities in Upper Mesopotamia during the 7th

757 and 6th millennia BCE estimated using the AH-RJMCMC method (Livermore et al., 2018).

758 (a) The data available in the Near East are shown using their prior dating. The variation curve

759 (median values) and its 95\% credible interval are in blue. The orange curve and shaded area

760 show the evolution of the intensities derived from the Balkan data transferred to the latitude

761 of Tell Halaf and assigning to these data minimum age uncertainties of \pm 50 years. (b) Same as

762 in (a) but the data are exhibited using the posterior probability distributions of their age

763 values.

764 Fig. S4. Behavior in geomagnetic field intensity as derived from the Upper Mesopotamian 765 data (Gallet et al., 2015; Yutsis-Akimova et al., 2018a,b; this study). All data are transferred 766 to the latitude of Tell Halaf, Syria. (a,b) Same as in Fig. 2 but the time order relationship

767 between the data from Yarim Tepe II concerns 14 non-overlapping stratigraphic layers,

768 instead of nine archeological levels (see text). (c,d) Same as in Fig. 2 but the archeological 769 transitions inside the Yarim Tepe II sequence are ignored for the AH-RJMCMC calculations 770 (see Section 3b). 
771 Table S1. Different datasets from the Balkans and the Near East used for AH-RJMCMC

772 modeling (see text and details in the table).

773 Table S2. New archeointensity data obtained at Tell Begum. Mean intensities are first 774 estimated at the specimen level, then at the fragment level, and finally at the fragment group 775 level. This last group-mean value is used for the AH-RJMCMC modeling, with a dating of

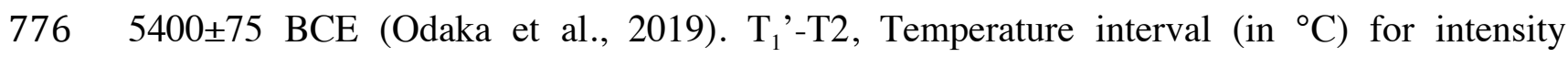
777 determination; Hlab, laboratory field used for TRM acquisition; NRM $\mathrm{T}_{1}{ }^{\prime}(\%)$, fraction of 778 NRM involved in intensity determination; Slope R' (\%), slope of the R'(Ti) data within the 779 temperature interval used for intensity determination; $\mathrm{F}$, intensity value in $\mu \mathrm{T}$ derived per 780 specimen; F mean value per fragment $\pm \sigma$, mean intensity in $\mu \mathrm{T}$ computed per fragment with 781 its standard deviation. Group $\mathrm{F}$ mean value $\pm \sigma$, mean intensity in $\mu \mathrm{T}$ computed for each 782 group of fragments.

783 Table S3. Medians and 95\% credible intervals estimated using the AH-RJMCMC method 784 (Livermore et al. 2018) and associated rates of changes (based on the time derivative of the 785 medians) shown in Figs. 1, 2, 3, S2 and S4. The datasets are those provided in Table S1. See 786 text 

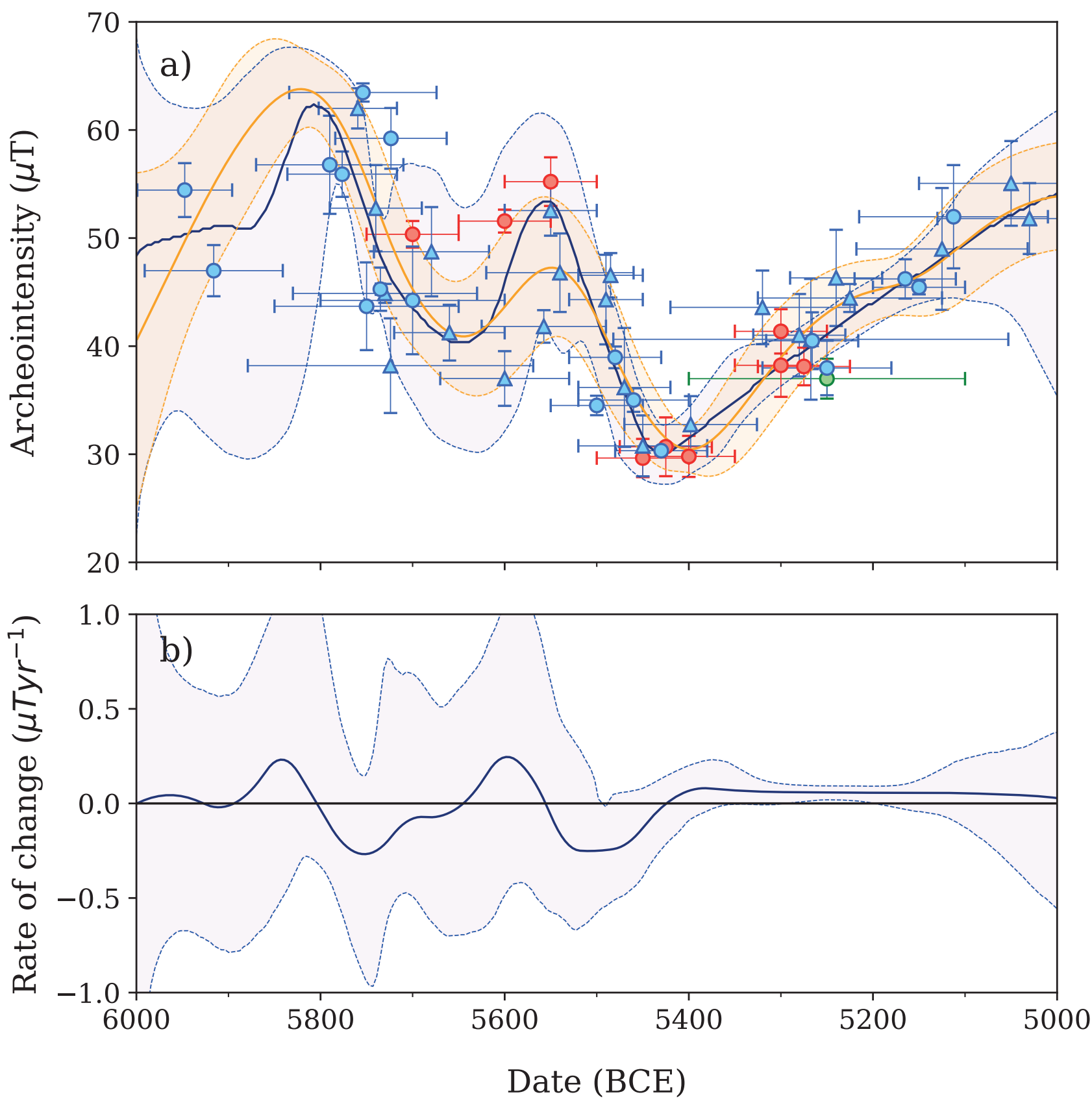

Figure 1 


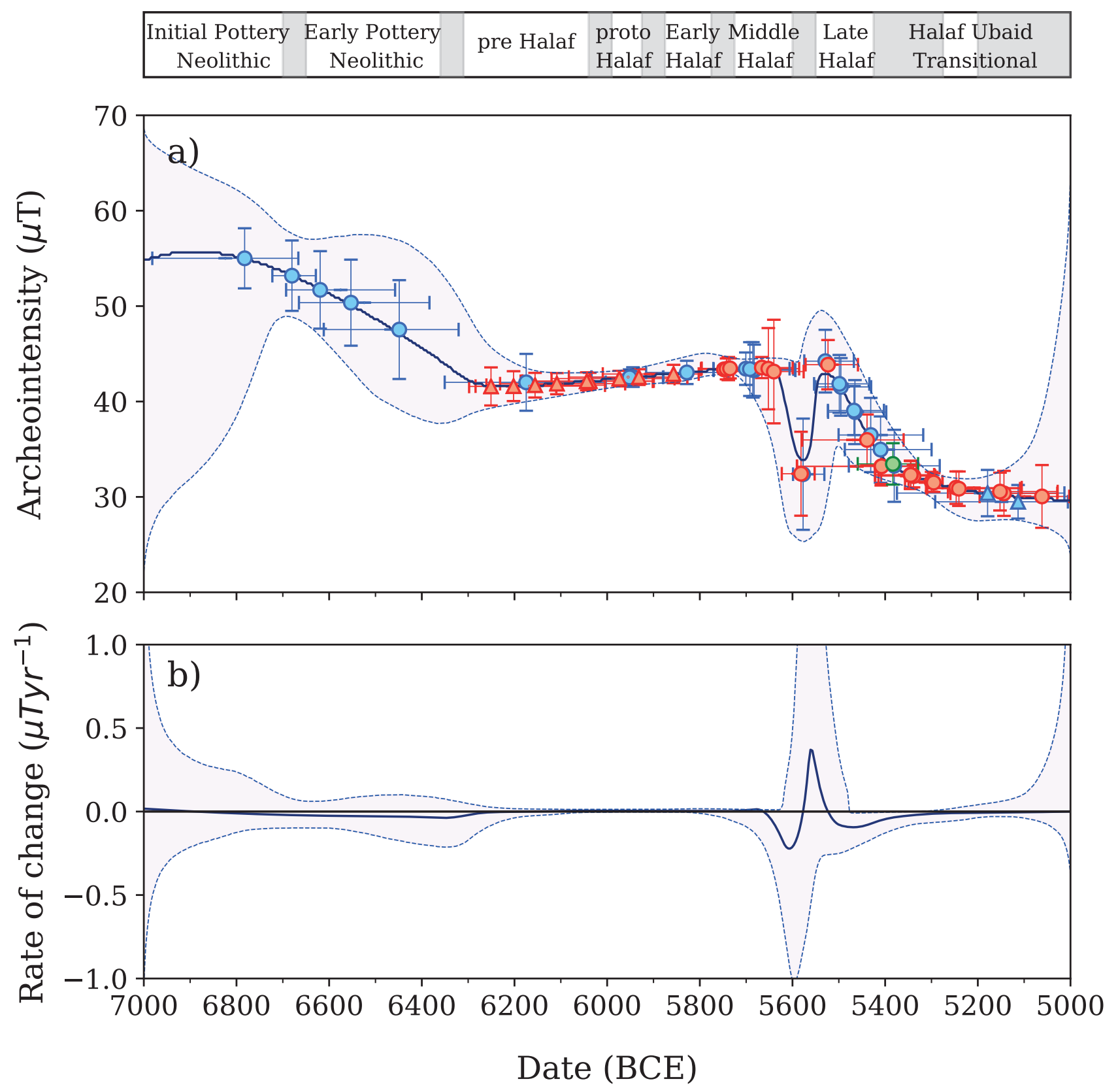

Figure 2 


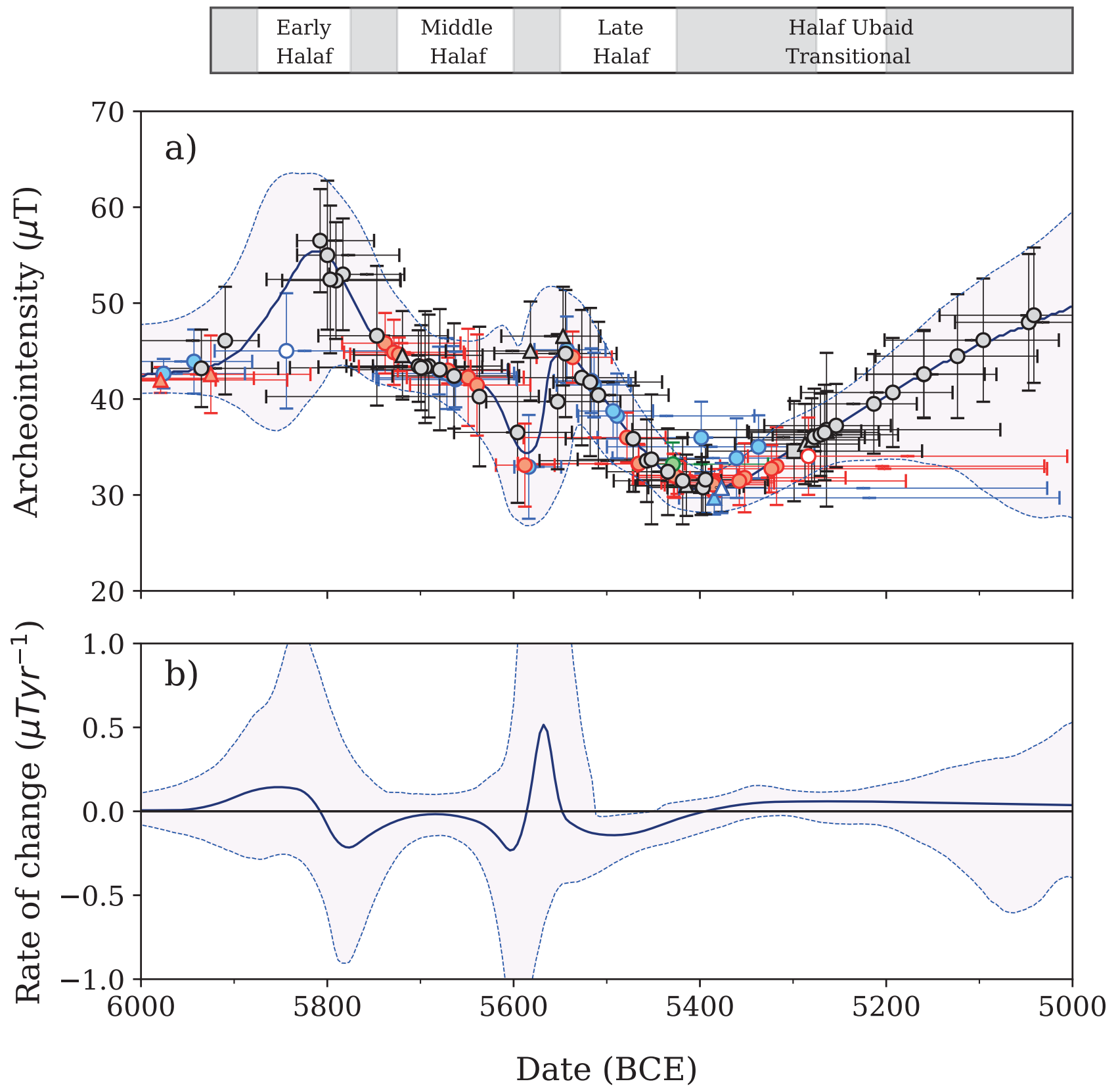

Figure 3 
a) $S Y 91$

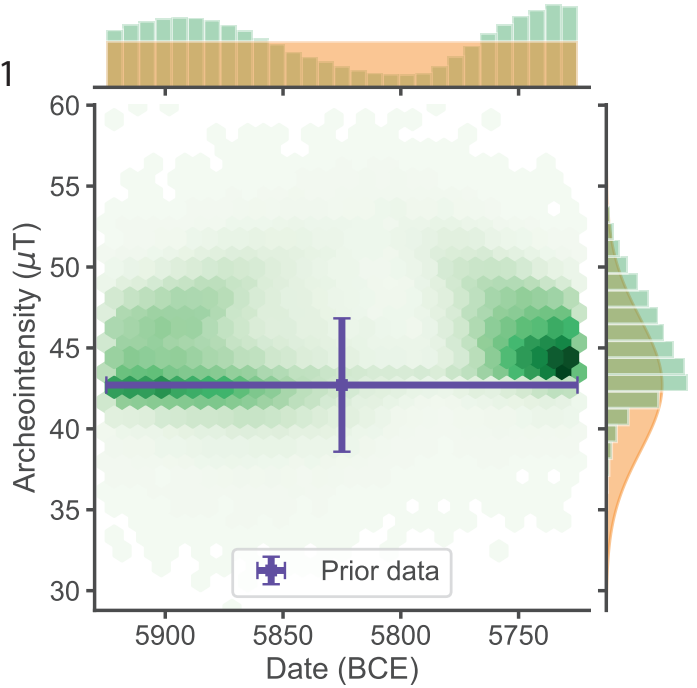

b) $\mathrm{SY} 37$

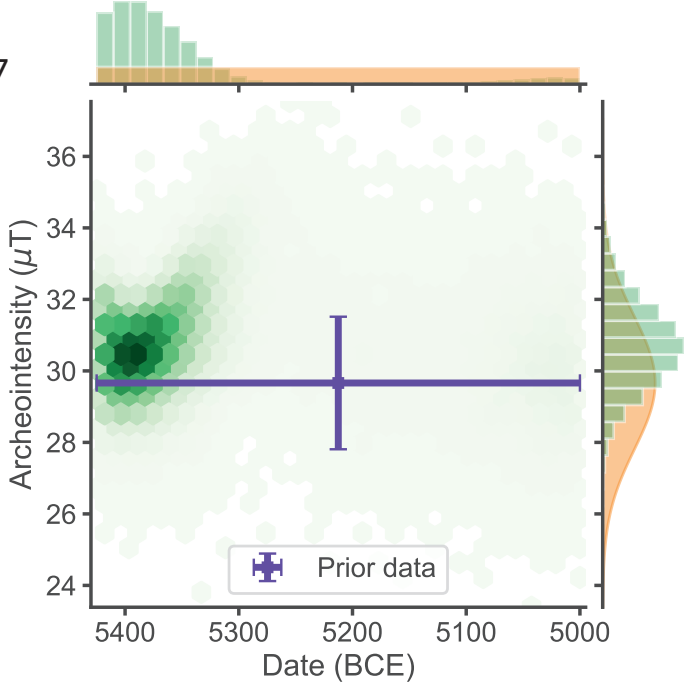

c) YT01

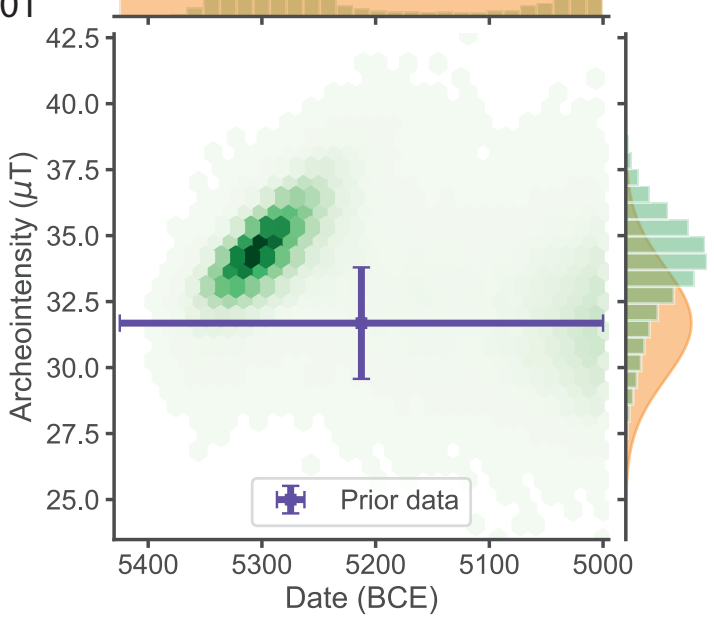

Figure 4 


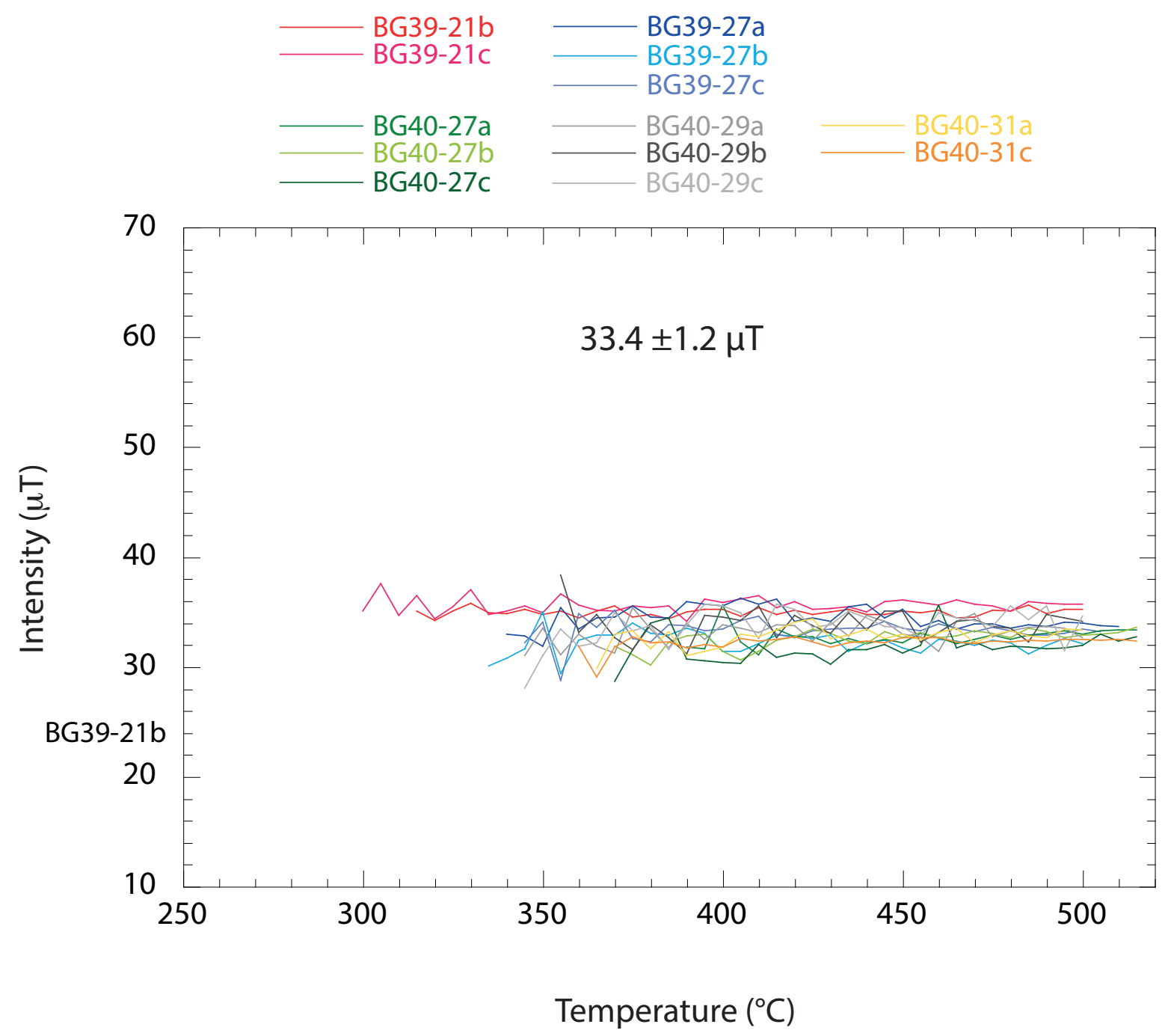

Figure S1 

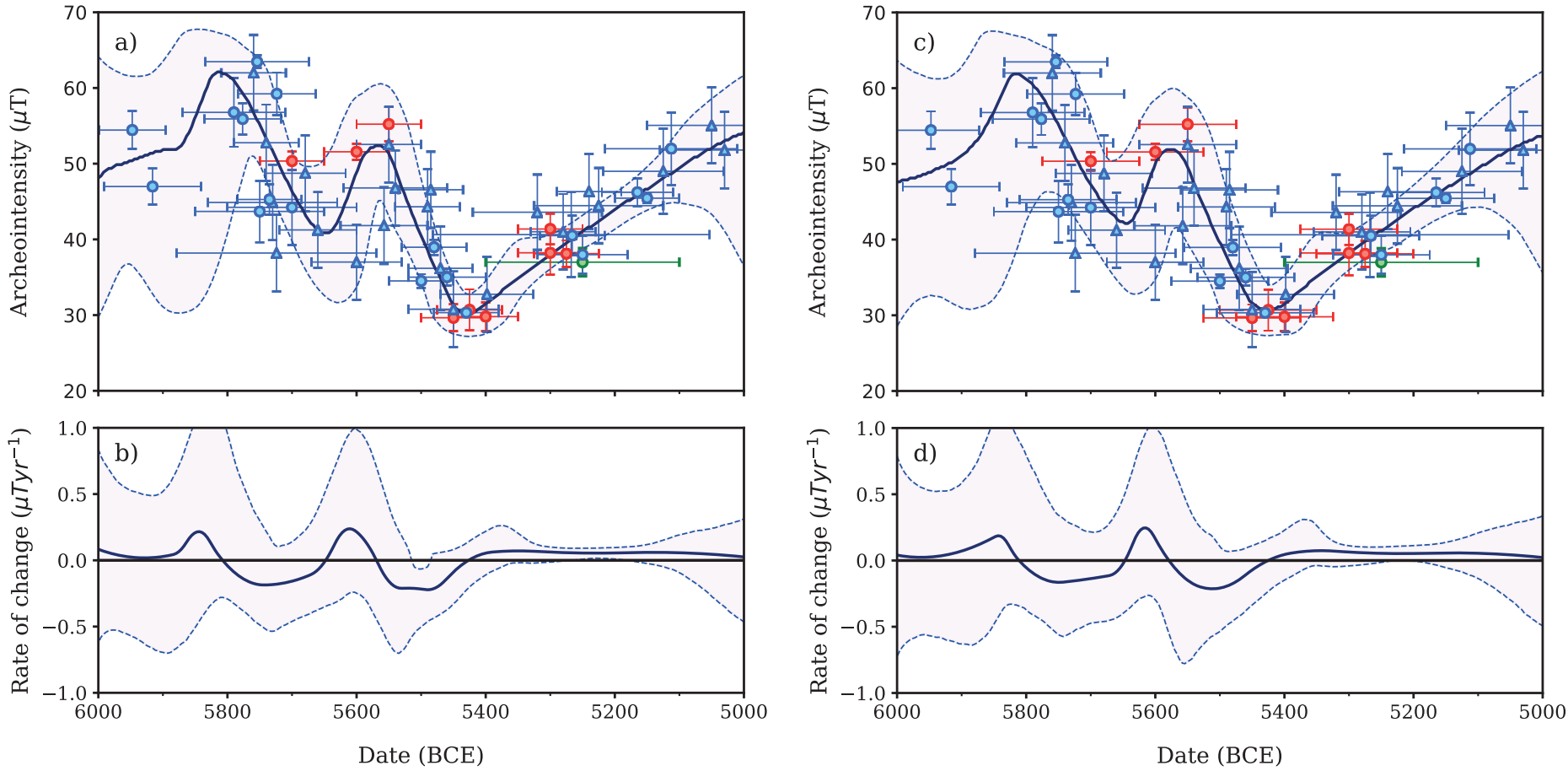

Figure S2 

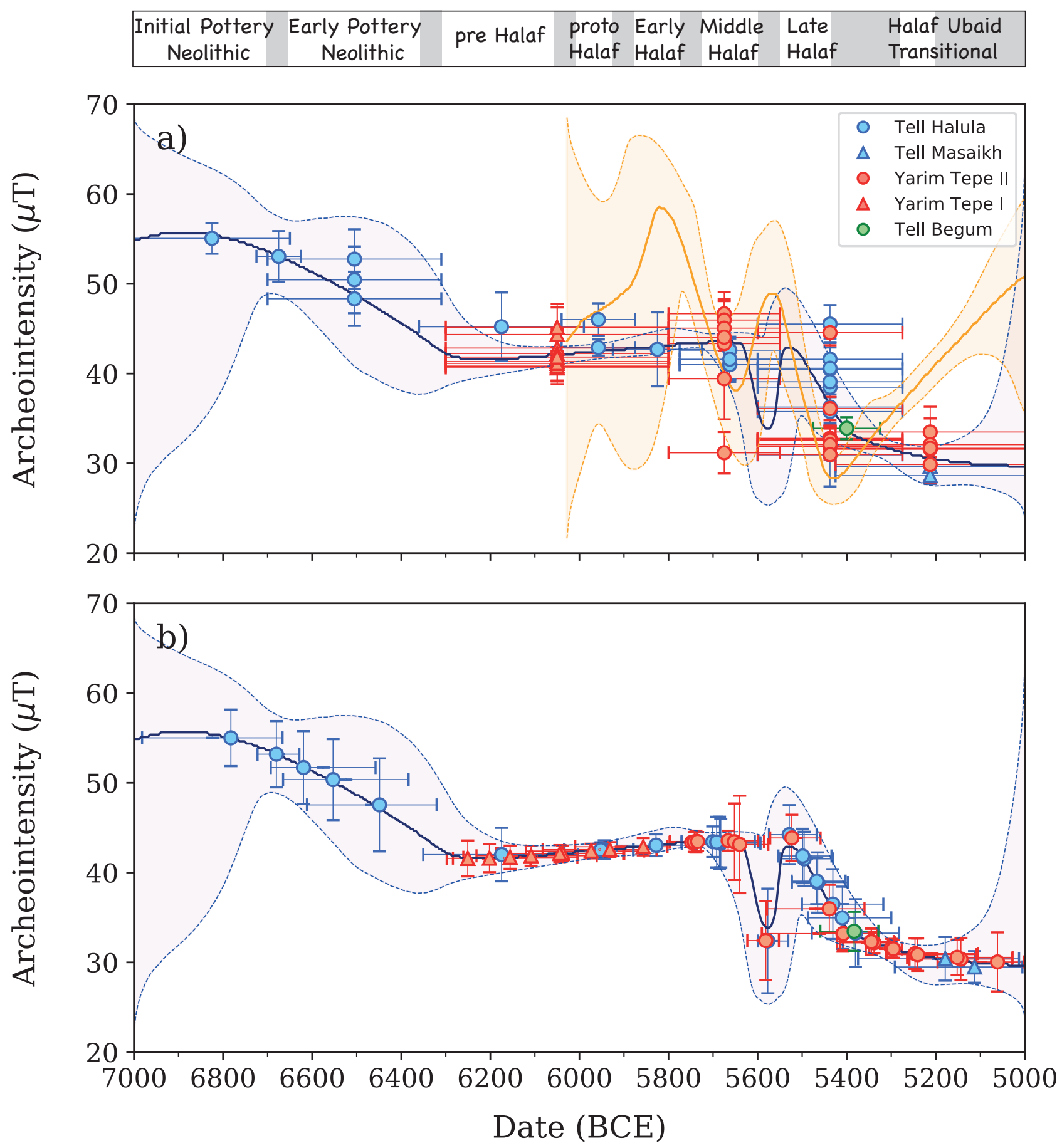

Figure S3 

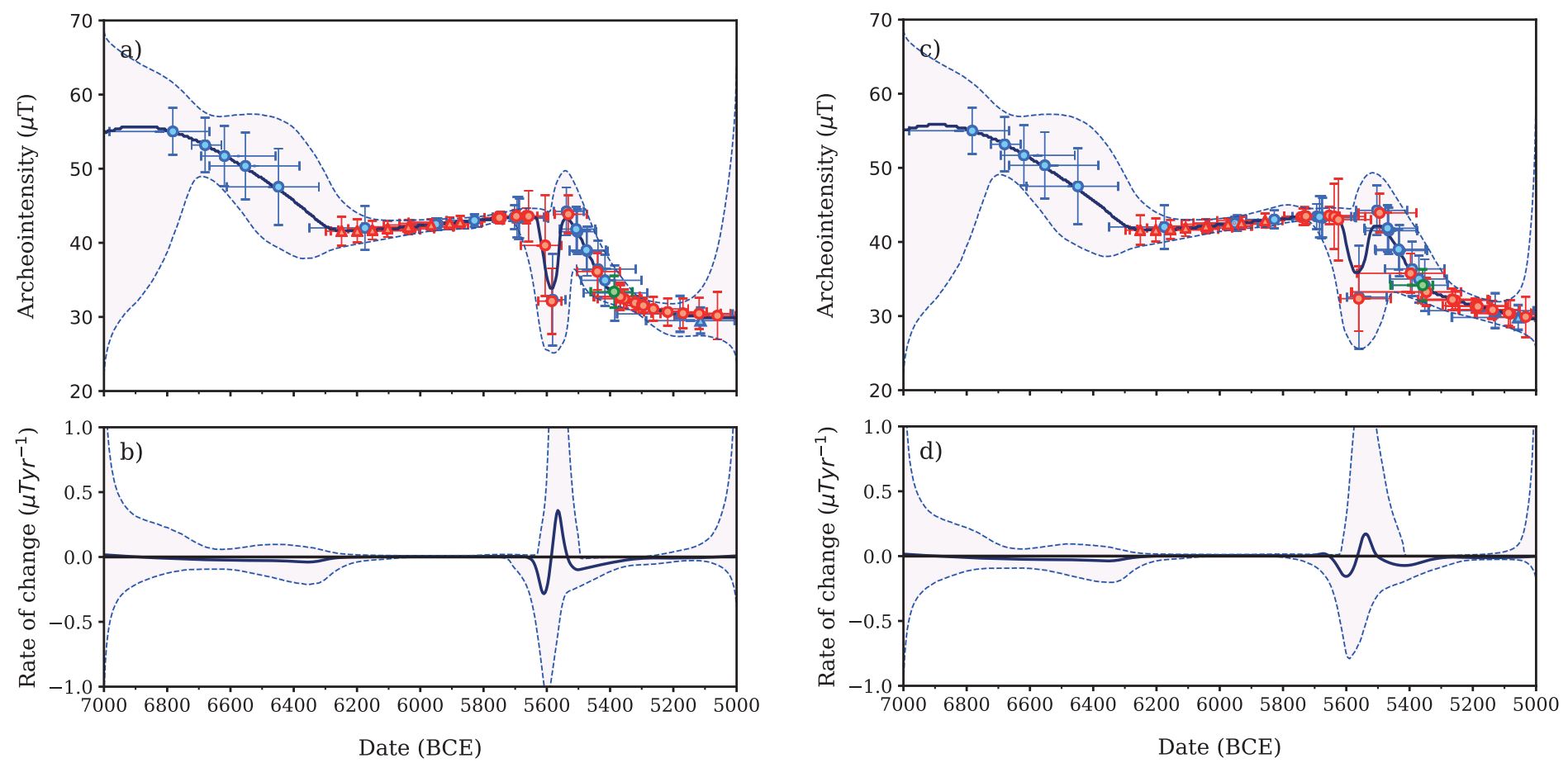

Figure S4 


\section{Table S1}

(All data transferred to the latitude of Sofia, Bulgaria)

Data Fig. 1

Ref.

Date

$d t$

Intensity

sd

Order

Fanjat et al. (2013)

\begin{tabular}{|c|c|c|c|c|}
\hline AVGI & -5250.0 & 150.00 & 36.992 & 1.8444 \\
\hline \multicolumn{5}{|c|}{ Kostadinova-Avramova et al. (2019) } \\
\hline Ilindentsi & -5700.0 & 50.000 & 50.35 & 1.23 \\
\hline Site15-II & -5600.0 & 50.000 & 51.57 & 1.06 \\
\hline Site15-I & -5550.0 & 50.000 & 55.22 & 2.25 \\
\hline Site14 & -5425.0 & 50.000 & 30.69 & 2.72 \\
\hline Chavd1 & -5450.0 & 50.000 & 29.65 & 1.77 \\
\hline Chavd2 & -5400.0 & 50.000 & 29.8 & 1.9 \\
\hline Chavd3 & -5300.0 & 50.000 & 38.23 & 2.92 \\
\hline Site16 & -5300.0 & 50.000 & 41.37 & 2.05 \\
\hline Site15-BDR & -5275.0 & 50.000 & 38.12 & 1.74 \\
\hline
\end{tabular}

Kovacheva et al. (2014)

$\begin{array}{llllll}\text { no09 } & -5660.0 & 60.000 & 41.249 & 2.5787 & 1 \mathrm{c} \\ \text { no10 } & -5600.0 & 70.000 & 37.008 & 2.5289 & 2 \mathrm{c} \\ \text { no354 } & -5540.0 & 80.000 & 46.795 & 3.6341 & 3 \mathrm{c} \\ \text { no355 } & -5450.0 & 70.000 & 30.775 & 2.8177 & 4 \mathrm{c} \\ \text { no19 } & -5320.0 & 100.00 & 43.589 & 3.4051 & 5 \mathrm{c} \\ \text { no17 } & -5225.0 & 100.00 & 44.455 & 1.2943 & 6 \mathrm{c} \\ \text { no13 } & -5490.0 & 40.000 & 44.309 & 4.1445 & 1 \mathrm{~d} \\ \text { no18 } & -5267.5 & 214.50 & 40.635 & 5.5861 & 2 \mathrm{~d} \\ \text { no358 } & -5500.0 & 50.000 & 34.513 & 0.90017 & 1 \mathrm{e} \\ \text { no294 } & -5430.0 & 50.000 & 30.329 & 0.37590 & 2 \mathrm{e} \\ \text { no293 } & -5150.0 & 50.000 & 45.444 & 0.65287 & 3 \mathrm{e} \\ \text { no250 } & -5916.0 & 75.000 & 46.985 & 2.3678 & 1 \mathrm{f} \\ \text { no249 } & -5754.0 & 80.000 & 63.469 & 0.84277 & 2 \mathrm{f} \\ \text { no11 } & -5723.5 & 60.500 & 59.225 & 2.8093 & 3 \mathrm{f} \\ \text { no26 } & -5030.0 & 100.00 & 51.806 & 3.2789 & 0 \\ \text { no25 } & -5050.0 & 100.00 & 55.061 & 3.9186 & 0 \\ \text { no260 } & -5112.5 & 102.50 & 51.975 & 4.7785 & 0 \\ \text { no24 } & -5125.0 & 93.000 & 48.995 & 5.6353 & 0 \\ \text { no302 } & -5165.0 & 55.000 & 46.220 & 1.8172 & 0 \\ \text { no16 } & -5240.0 & 50.000 & 46.316 & 4.4508 & 0 \\ \text { no263 } & -5250.0 & 70.000 & 37.981 & 2.5234 & 0 \\ \text { no353 } & -5266.0 & 50.000 & 40.501 & 2.6291 & 0 \\ \text { no23 } & -5280.0 & 50.000 & 41.010 & 3.8133 & 0 \\ \text { no05 } & -5398.0 & 72.000 & 32.751 & 2.6256 & 0 \\ \text { no326 } & -5460.0 & 60.000 & 35.013 & 1.0875 & 0 \\ \text { no06 } & -5470.0 & 50.000 & 36.181 & 5.5019 & 0 \\ \text { no262 } & -5480.0 & 50.000 & 38.964 & 1.0034 & 0 \\ \text { no14 } & -5485.0 & 35.000 & 46.557 & 2.0535 & 0 \\ \text { no259 } & -5550.0 & 50.000 & 52.545 & 2.3251 & 0\end{array}$




$\begin{array}{lccccc}\text { no08 } & -5557.5 & 67.500 & 41.824 & 1.5049 & 0 \\ \text { no07 } & -5679.5 & 62.500 & 48.737 & 4.1291 & 0 \\ \text { no324 } & -5700.0 & 100.00 & 44.234 & 4.9819 & 0 \\ \text { no261 } & -5724.0 & 155.00 & 38.195 & 4.3779 & 0 \\ \text { no04 } & -5730.0 & 100.00 & 44.880 & 0.88000 & 0 \\ \text { no241 } & -5735.0 & 5.0000 & 45.270 & 2.0000 & 0 \\ \text { no02 } & -5740.0 & 50.000 & 52.766 & 3.9907 & 0 \\ \text { no139 } & -5750.0 & 100.00 & 43.693 & 4.0581 & 0 \\ \text { no242 } & -5759.5 & 42.500 & 62.000 & 1.8600 & 0 \\ \text { no243 } & -5776.5 & 59.500 & 55.912 & 2.0985 & 0 \\ \text { no246 } & -5790.0 & 80.000 & 56.779 & 4.5399 & 0 \\ \text { no247 } & -5947.5 & 51.500 & 54.440 & 2.4929 & 0\end{array}$

Column "Order": 0 indicates the absence of a time-order relationhip in the AH-RJMCMC calculations 
(All data transferred to the latitude of Tell Halaf, Syria)

Data Fig. 2

Date

$\mathrm{dt}$

Intensity sd

order

Gallet et al. (2015)

$\begin{array}{llllll}\text { SY127 } & -6825.0 & 175.00 & 55.066 & 1.7083 & 1 \mathrm{a} \\ \text { SY125 } & -6675.0 & 50.000 & 53.057 & 2.8136 & 2 \mathrm{a} \\ \text { SY98-128 } & -6505.0 & 195.00 & 50.444 & 3.7180 & 3 a \\ \text { SY97-129 } & -6505.0 & 195.00 & 52.755 & 3.3160 & 4 a \\ \text { SY96 } & -6505.0 & 195.00 & 48.334 & 3.0146 & 5 a \\ \text { SY130 } & -6175.0 & 185.00 & 45.219 & 3.8185 & 6 a \\ \text { SY94-137 } & -5957.5 & 82.500 & 46.023 & 1.8088 & 7 a \\ \text { SY95 } & -5957.5 & 82.500 & 42.908 & 0.90438 & 7 a \\ \text { SY91 } & -5825.0 & 100.00 & 42.707 & 4.1199 & 8 a \\ \text { SY87 } & -5662.5 & 112.50 & 40.998 & 1.6078 & 9 a \\ \text { SY88 } & -5662.5 & 112.50 & 42.606 & 1.5073 & 9 a \\ \text { SY89 } & -5662.5 & 112.50 & 40.998 & 1.9092 & 9 a \\ \text { SY90 } & -5662.5 & 112.50 & 41.601 & 2.3112 & 9 a \\ \text { SY86-131 } & -5437.5 & 162.50 & 30.950 & 3.5170 & 10 a \\ \text { SY135 } & -5437.5 & 162.50 & 45.520 & 2.1102 & 11 a \\ \text { SY84 } & -5437.5 & 162.50 & 40.496 & 2.4117 & 12 a \\ \text { SY138 } & -5437.5 & 162.50 & 41.601 & 2.3112 & 12 a \\ \text { SY82 } & -5437.5 & 162.50 & 38.486 & 2.8136 & 13 a \\ \text { SY83-136 } & -5437.5 & 162.50 & 39.089 & 1.7083 & 13 a \\ \text { SY80 } & -5437.5 & 162.50 & 35.773 & 3.5170 & 14 a \\ \text { SY81 } & -5437.5 & 162.50 & 36.276 & 2.0097 & 14 a \\ \text { SY132 } & -5437.5 & 162.50 & 40.596 & 2.9141 & 14 a \\ \text { SY37 } & -5212.5 & 212.50 & 29.662 & 1.8539 & 0 \\ \text { SY38 } & -5212.5 & 212.50 & 28.632 & 0.92693 & 0\end{array}$

Yutsis-Akimova et al. (2018a)

$\begin{array}{llllll}\text { YT22 } & -5675.0 & 125.00 & 43.352 & 0.20117 & 1 \mathrm{~b} \\ \text { YT28 } & -5675.0 & 125.00 & 46.671 & 1.6093 & 1 \mathrm{~b} \\ \text { YT27 } & -5675.0 & 125.00 & 45.967 & 2.1123 & 1 \mathrm{~b} \\ \text { YT26 } & -5675.0 & 125.00 & 45.062 & 0.90526 & 1 \mathrm{~b} \\ \text { YT25 } & -5675.0 & 125.00 & 43.352 & 0.80467 & 2 \mathrm{~b} \\ \text { YT24 } & -5675.0 & 125.00 & 44.056 & 5.0292 & 2 \mathrm{~b} \\ \text { YT23 } & -5675.0 & 125.00 & 39.429 & 4.5263 & 2 \mathrm{~b} \\ \text { YT22A } & -5675.0 & 125.00 & 31.181 & 2.3134 & 3 \mathrm{~b} \\ \text { YT22 } & -5437.5 & 162.50 & 44.559 & 1.4082 & 4 \mathrm{~b} \\ \text { YT21 } & -5437.5 & 162.50 & 36.110 & 1.3076 & 4 \mathrm{~b} \\ \text { YT20 } & -5437.5 & 162.50 & 32.690 & 1.2070 & 4 \mathrm{~b} \\ \text { YT19 } & -5437.5 & 162.50 & 32.790 & 1.1064 & 4 \mathrm{~b} \\ \text { YT17 } & -5437.5 & 162.50 & 32.690 & 1.5088 & 5 \mathrm{~b} \\ \text { YT16 } & -5437.5 & 162.50 & 31.885 & 1.0058 & 5 \mathrm{~b} \\ \text { YT15 } & -5437.5 & 162.50 & 32.589 & 1.5088 & 5 \mathrm{~b} \\ \text { YT14 } & -5437.5 & 162.50 & 32.589 & 1.6093 & 5 \mathrm{~b} \\ \text { YT11 } & -5437.5 & 162.50 & 32.086 & 1.1064 & 6 \mathrm{~b} \\ \text { YT10 } & -5437.5 & 162.50 & 30.980 & 0.60350 & 6 \mathrm{~b} \\ \text { YT07 } & -5212.5 & 212.50 & 33.494 & 2.8163 & 7 \mathrm{~b}\end{array}$




$\begin{array}{llllll}\text { YT06 } & -5212.5 & 212.50 & 29.874 & 2.0117 & 7 \mathrm{~b} \\ \text { YT04 } & -5212.5 & 212.50 & 31.583 & 3.4199 & 8 \mathrm{~b} \\ \text { YT03 } & -5212.5 & 212.50 & 32.086 & 1.4082 & 8 \mathrm{~b} \\ \text { YT01 } & -5212.5 & 212.50 & 31.684 & 2.1123 & 9 \mathrm{~b}\end{array}$

Yutsis-Akimova et al. (2018b)

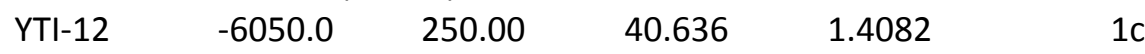

$\begin{array}{lllll}\text { YTI-11 } & -6050.0 & 250.00 & 42.849 & 2.2129\end{array}$

$\begin{array}{lllll}\text { YTI-10 } & -6050.0 & 250.00 & 40.837 & 1.7099\end{array}$

$\begin{array}{lllll}\text { YTI-08A } & -6050.0 & 250.00 & 44.358 & 3.4199\end{array}$

$\begin{array}{llllll}\text { YTI-06 } & -6050.0 & 250.00 & 42.245 & 0.70409 & \text { 5c }\end{array}$

$\begin{array}{llllll}\text { YTI-05 } & -6050.0 & 250.00 & 41.340 & 1.2070 & 5 \mathrm{c}\end{array}$

$\begin{array}{lllll}\text { YTI-03 } & -6050.0 & 250.00 & 45.162 & 2.2129\end{array}$

$\begin{array}{lllll}\text { YTI-02A } & -6050.0 & 250.00 & 41.139 & 0.70409\end{array}$

$\begin{array}{lllll}\text { YTI-01 } & -6050.0 & 250.00 & 41.843 & 3.0175\end{array}$

This study

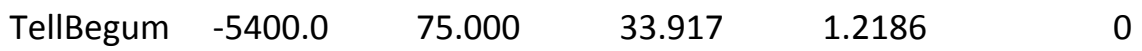

Column "Order": 0 indicates the absence of a time-order relationhip in the AH-RJMCMC calculations 
(All data transferred to the latitude of Tell Halaf, Syria)

Data Fig. 3

\begin{tabular}{|c|c|c|c|c|c|}
\hline Ref. & Date & $\mathrm{dt}$ & Intensity & sd & order \\
\hline \multicolumn{6}{|l|}{ Near East } \\
\hline SY127 & -6825.0 & 175.00 & 55.066 & 1.7083 & $1 a$ \\
\hline SY125 & -6675.0 & 50.000 & 53.057 & 2.8136 & $2 a$ \\
\hline SY98-128 & -6505.0 & 195.00 & 50.444 & 3.7180 & $3 a$ \\
\hline SY97-129 & -6505.0 & 195.00 & 52.755 & 3.3160 & $4 a$ \\
\hline SY96 & -6505.0 & 195.00 & 48.334 & 3.0146 & $5 a$ \\
\hline SY130 & -6175.0 & 185.00 & 45.219 & 3.8185 & $6 a$ \\
\hline SY94-137 & -5957.5 & 82.500 & 46.023 & 1.8088 & $7 a$ \\
\hline SY95 & -5957.5 & 82.500 & 42.908 & 0.90438 & $7 a$ \\
\hline SY91 & -5825.0 & 100.00 & 42.707 & 4.1199 & $8 a$ \\
\hline SY87 & -5662.5 & 112.50 & 40.998 & 1.6078 & $9 a$ \\
\hline SY88 & -5662.5 & 112.50 & 42.606 & 1.5073 & $9 a$ \\
\hline SY89 & -5662.5 & 112.50 & 40.998 & 1.9092 & $9 a$ \\
\hline SY9O & -5662.5 & 112.50 & 41.601 & 2.3112 & $9 a$ \\
\hline SY86-131 & -5437.5 & 162.50 & 30.950 & 3.5170 & $10 a$ \\
\hline SY135 & -5437.5 & 162.50 & 45.520 & 2.1102 & $11 a$ \\
\hline SY84 & -5437.5 & 162.50 & 40.496 & 2.4117 & $12 a$ \\
\hline SY138 & -5437.5 & 162.50 & 41.601 & 2.3112 & $12 a$ \\
\hline SY82 & -5437.5 & 162.50 & 38.486 & 2.8136 & $13 a$ \\
\hline SY83-136 & -5437.5 & 162.50 & 39.089 & 1.7083 & $13 a$ \\
\hline SY80 & -5437.5 & 162.50 & 35.773 & 3.5170 & $14 a$ \\
\hline SY81 & -5437.5 & 162.50 & 36.276 & 2.0097 & $14 a$ \\
\hline SY132 & -5437.5 & 162.50 & 40.596 & 2.9141 & $14 a$ \\
\hline SY37 & -5212.5 & 212.50 & 29.662 & 1.8539 & 0 \\
\hline SY38 & -5212.5 & 212.50 & 28.632 & 0.92693 & 0 \\
\hline YT29 & -5675.0 & 125.00 & 43.352 & 0.20117 & $1 b$ \\
\hline YT28 & -5675.0 & 125.00 & 46.671 & 1.6093 & $1 b$ \\
\hline YT27 & -5675.0 & 125.00 & 45.967 & 2.1123 & $1 b$ \\
\hline YT26 & -5675.0 & 125.00 & 45.062 & 0.90526 & $1 b$ \\
\hline YT25 & -5675.0 & 125.00 & 43.352 & 0.80467 & $2 b$ \\
\hline YT24 & -5675.0 & 125.00 & 44.056 & 5.0292 & $2 b$ \\
\hline YT23 & -5675.0 & 125.00 & 39.429 & 4.5263 & $2 b$ \\
\hline YT22A & -5675.0 & 125.00 & 31.181 & 2.3134 & $3 b$ \\
\hline YT22 & -5437.5 & 162.50 & 44.559 & 1.4082 & $4 b$ \\
\hline YT21 & -5437.5 & 162.50 & 36.110 & 1.3076 & $4 b$ \\
\hline YT20 & -5437.5 & 162.50 & 32.690 & 1.2070 & $4 b$ \\
\hline YT19 & -5437.5 & 162.50 & 32.790 & 1.1064 & $4 b$ \\
\hline YT17 & -5437.5 & 162.50 & 32.690 & 1.5088 & $5 b$ \\
\hline YT16 & -5437.5 & 162.50 & 31.885 & 1.0058 & $5 b$ \\
\hline YT15 & -5437.5 & 162.50 & 32.589 & 1.5088 & $5 b$ \\
\hline YT14 & -5437.5 & 162.50 & 32.589 & 1.6093 & $5 b$ \\
\hline YT11 & -5437.5 & 162.50 & 32.086 & 1.1064 & $6 b$ \\
\hline YT10 & -5437.5 & 162.50 & 30.980 & 0.60350 & $6 b$ \\
\hline YT07 & -5212.5 & 212.50 & 33.494 & 2.8163 & $7 b$ \\
\hline YT06 & -5212.5 & 212.50 & 29.874 & 2.0117 & $7 b$ \\
\hline YT04 & -5212.5 & 212.50 & 31.583 & 3.4199 & $8 b$ \\
\hline
\end{tabular}




\begin{tabular}{|c|c|c|c|c|c|}
\hline YT03 & -5212.5 & 212.50 & 32.086 & 1.4082 & $8 b$ \\
\hline YT01 & -5212.5 & 212.50 & 31.684 & 2.1123 & $9 b$ \\
\hline YTI-12 & -6050.0 & 250.00 & 40.636 & 1.4082 & $1 c$ \\
\hline YTI-11 & -6050.0 & 250.00 & 42.849 & 2.2129 & $2 c$ \\
\hline YTI-10 & -6050.0 & 250.00 & 40.837 & 1.7099 & $3 c$ \\
\hline YTI-08A & -6050.0 & 250.00 & 44.358 & 3.4199 & $4 c$ \\
\hline YTI-06 & -6050.0 & 250.00 & 42.245 & 0.70409 & $5 c$ \\
\hline YTI-05 & -6050.0 & 250.00 & 41.340 & 1.2070 & $5 c$ \\
\hline YTI-03 & -6050.0 & 250.00 & 45.162 & 2.2129 & $6 c$ \\
\hline YTI-02A & -6050.0 & 250.00 & 41.139 & 0.70409 & $7 c$ \\
\hline YTI-01 & -6050.0 & 250.00 & 41.843 & 3.0175 & $8 c$ \\
\hline TellBegum & -5400.0 & 75.000 & 33.917 & 1.2186 & 0 \\
\hline \multicolumn{6}{|l|}{ Balkans } \\
\hline AVGI & -5250.0 & 150.00 & 34.563 & 3.0000 & 0 \\
\hline Ilindentsi & -5700.0 & 75.000 & 47.046 & 3.0000 & 0 \\
\hline Site15-II & -5600.0 & 75.000 & 48.183 & 3.0000 & $1 d$ \\
\hline Site15-I & -5550.0 & 75.000 & 51.597 & 3.0000 & $2 d$ \\
\hline Site14 & -5425.0 & 75.000 & 28.671 & 3.0000 & 0 \\
\hline Chavd1 & -5450.0 & 75.000 & 27.692 & 3.0000 & $1 \mathrm{e}$ \\
\hline Chavd2 & -5400.0 & 75.000 & 27.834 & 3.0000 & $2 \mathrm{e}$ \\
\hline Chavd3 & -5300.0 & 75.000 & 35.712 & 3.0000 & $3 e$ \\
\hline Site16 & -5300.0 & 75.000 & 38.665 & 3.0000 & 0 \\
\hline Site15-BDR & -5275.0 & 75.000 & 35.611 & 3.0000 & 0 \\
\hline no09 & -5660.0 & 75.000 & 38.541 & 4.6514 & $1 f$ \\
\hline no10 & -5600.0 & 75.000 & 34.578 & 4.6514 & $2 f$ \\
\hline no354 & -5540.0 & 80.000 & 43.723 & 4.6514 & $3 f$ \\
\hline no355 & -5450.0 & 75.000 & 28.755 & 4.6514 & $4 f$ \\
\hline no19 & -5320.0 & 100.00 & 40.727 & 4.6514 & $5 f$ \\
\hline no17 & -5225.0 & 100.00 & 41.537 & 4.6514 & $6 f$ \\
\hline no13 & -5490.0 & 75.000 & 41.400 & 4.6769 & $1 g$ \\
\hline no18 & -5267.5 & 214.50 & 37.967 & 5.2194 & $2 g$ \\
\hline no358 & -5500.0 & 75.000 & 32.247 & 3.0000 & $1 \mathrm{~h}$ \\
\hline no294 & -5430.0 & 75.000 & 28.338 & 3.0000 & $2 \mathrm{~h}$ \\
\hline no293 & -5150.0 & 75.000 & 42.460 & 3.0000 & $3 \mathrm{~h}$ \\
\hline no250 & -5916.0 & 75.000 & 43.900 & 3.0000 & $1 \mathrm{i}$ \\
\hline no249 & -5754.0 & 80.000 & 59.302 & 3.0000 & $2 \mathrm{i}$ \\
\hline no11 & -5723.5 & 75.000 & 55.337 & 3.0000 & $3 i$ \\
\hline no26 & -5030.0 & 100.00 & 48.405 & 4.7132 & 0 \\
\hline no25 & -5050.0 & 100.00 & 51.446 & 4.6820 & 0 \\
\hline no260 & -5112.5 & 102.50 & 48.562 & 4.4647 & 0 \\
\hline no24 & -5125.0 & 93.000 & 45.779 & 5.2653 & 0 \\
\hline no302 & -5165.0 & 75.000 & 43.186 & 3.0000 & 0 \\
\hline no16 & -5240.0 & 75.000 & 43.275 & 4.6413 & 0 \\
\hline no263 & -5250.0 & 75.000 & 35.487 & 3.0000 & 0 \\
\hline no353 & -5266.0 & 75.000 & 37.842 & 3.0000 & 0 \\
\hline no23 & -5280.0 & 75.000 & 38.318 & 4.6514 & 0 \\
\hline no05 & -5398.0 & 75.000 & 30.601 & 4.6463 & 0 \\
\hline no326 & -5460.0 & 75.000 & 32.714 & 3.0000 & 0 \\
\hline no06 & -5470.0 & 75.000 & 33.806 & 5.1407 & 0 \\
\hline no262 & -5480.0 & 75.000 & 36.406 & 3.0000 & 0 \\
\hline
\end{tabular}




$\begin{array}{llllll}\text { no14 } & -5485.0 & 75.000 & 43.500 & 4.7028 & 0 \\ \text { no259 } & -5550.0 & 75.000 & 49.096 & 4.6820 & 0 \\ \text { no08 } & -5557.5 & 75.000 & 39.078 & 4.7185 & 0 \\ \text { no07 } & -5679.5 & 75.000 & 45.537 & 4.6820 & 0 \\ \text { no324 } & -5700.0 & 100.00 & 41.330 & 4.6548 & 0 \\ \text { no261 } & -5724.0 & 155.00 & 35.687 & 4.7343 & 0 \\ \text { no04 } & -5730.0 & 100.00 & 41.933 & 4.6717 & 0 \\ \text { no241 } & -5735.0 & 75.000 & 42.298 & 3.0000 & 0 \\ \text { no02 } & -5740.0 & 75.000 & 49.302 & 4.7080 & 0 \\ \text { no139 } & -5750.0 & 100.00 & 40.824 & 3.7917 & 0 \\ \text { no242 } & -5759.5 & 75.000 & 57.930 & 4.6717 & 0 \\ \text { no243 } & -5776.5 & 75.000 & 52.241 & 3.0000 & 0 \\ \text { no246 } & -5790.0 & 80.000 & 53.052 & 4.2419 & 0 \\ \text { no247 } & -5947.5 & 75.000 & 50.866 & 3.0000 & 0\end{array}$

Column "Order": 0 indicates the absence of a time-order relationhip in the AH-RJMCMC calculations 
(All data at the latitude of Sofia, Bulgaria)

\begin{tabular}{|c|c|c|c|c|c|c|c|c|c|c|}
\hline \multicolumn{2}{|c|}{ Data Fig. S2a,b } & & & & & \multicolumn{2}{|c|}{ Data Fig. S2c,d } & \multirow[b]{2}{*}{ Intensity } & \multirow[b]{2}{*}{ sd } & \multirow[b]{2}{*}{ Order } \\
\hline Ref. & Date & $\mathrm{dt}$ & Intensity & sd & Order & Date & $\mathrm{dt}$ & & & \\
\hline \multicolumn{11}{|c|}{ Fanjat et al. (2013) } \\
\hline AVGI & -5250.0 & 150.00 & 36.992 & 1.8444 & 0 & -5250.0 & 150.00 & 36.992 & 1.8444 & 0 \\
\hline \multicolumn{11}{|c|}{ Kostadinova-Avramova et al. (2019) } \\
\hline Ilindentsi & -5700.0 & 50.000 & 50.35 & 1.23 & 0 & -5700.0 & 75.000 & 50.35 & 1.23 & 0 \\
\hline Site15-II & -5600.0 & 50.000 & 51.57 & 1.06 & $1 \mathrm{a}$ & -5600.0 & 75.000 & 51.57 & 1.06 & $1 \mathrm{a}$ \\
\hline Site15-I & -5550.0 & 50.000 & 55.22 & 2.25 & $2 a$ & -5550.0 & 75.000 & 55.22 & 2.25 & $2 a$ \\
\hline Site14 & -5425.0 & 50.000 & 30.69 & 2.72 & 0 & -5425.0 & 75.000 & 30.69 & 2.72 & 0 \\
\hline Chavd1 & -5450.0 & 50.000 & 29.65 & 1.77 & $1 b$ & -5450.0 & 75.000 & 29.65 & 1.77 & $1 b$ \\
\hline Chavd2 & -5400.0 & 50.000 & 29.8 & 1.9 & $2 b$ & -5400.0 & 75.000 & 29.8 & 1.9 & $2 b$ \\
\hline Chavd3 & -5300.0 & 50.000 & 38.23 & 2.92 & $3 b$ & -5300.0 & 75.000 & 38.23 & 2.92 & $3 b$ \\
\hline Site16 & -5300.0 & 50.000 & 41.37 & 2.05 & 0 & -5300.0 & 75.000 & 41.37 & 2.05 & 0 \\
\hline Site15-BDR & -5275.0 & 50.000 & 38.12 & 1.74 & 0 & -5275.0 & 75.000 & 38.12 & 1.74 & 0 \\
\hline \multicolumn{11}{|c|}{ Kovacheva et al. (2014) } \\
\hline no09* & -5660.0 & 60.000 & 41.249 & 4.9782 & $1 c$ & -5660.0 & 75.000 & 41.249 & 4.9782 & $1 c$ \\
\hline no10* & -5600.0 & 70.000 & 37.008 & 4.9782 & $2 c$ & -5600.0 & 75.000 & 37.008 & 4.9782 & $2 c$ \\
\hline no354* & -5540.0 & 80.000 & 46.795 & 4.9782 & $3 c$ & -5540.0 & 80.000 & 46.795 & 4.9782 & $3 c$ \\
\hline no355* & -5450.0 & 70.000 & 30.775 & 4.9782 & $4 c$ & -5450.0 & 75.000 & 30.775 & 4.9782 & $4 c$ \\
\hline no19* & -5320.0 & 100.00 & 43.589 & 4.9782 & $5 c$ & -5320.0 & 100.00 & 43.589 & 4.9782 & $5 c$ \\
\hline no17* & -5225.0 & 100.00 & 44.455 & 4.9782 & $6 c$ & -5225.0 & 100.00 & 44.455 & 4.9782 & $6 c$ \\
\hline no13* & -5490.0 & 50.000 & 44.309 & 5.0055 & $1 d$ & -5490.0 & 75.000 & 44.309 & 5.0055 & $1 d$ \\
\hline no18* & -5267.5 & 214.50 & 40.635 & 5.5861 & $2 d$ & -5267.5 & 214.50 & 40.635 & 5.5861 & $2 d$ \\
\hline no358 & -5500.0 & 50.000 & 34.513 & 0.90017 & $1 e$ & -5500.0 & 75.000 & 34.513 & 0.90017 & $1 \mathrm{e}$ \\
\hline no294 & -5430.0 & 50.000 & 30.329 & 0.37590 & $2 e$ & -5430.0 & 75.000 & 30.329 & 0.37590 & $2 e$ \\
\hline no293 & -5150.0 & 50.000 & 45.444 & 0.65287 & $3 e$ & -5150.0 & 75.000 & 45.444 & 0.65287 & $3 e$ \\
\hline no250 & -5916.0 & 75.000 & 46.985 & 2.3678 & $1 f$ & -5916.0 & 75.000 & 46.985 & 2.3678 & $1 \mathrm{f}$ \\
\hline no249 & -5754.0 & 80.000 & 63.469 & 0.84277 & $2 f$ & -5754.0 & 80.000 & 63.469 & 0.84277 & $2 f$ \\
\hline no11 & -5723.5 & 60.500 & 59.225 & 2.8093 & $3 f$ & -5723.5 & 75.000 & 59.225 & 2.8093 & $3 f$ \\
\hline no26* & -5030.0 & 100.00 & 51.806 & 5.0444 & 0 & -5030.0 & 100.00 & 51.806 & 5.0444 & 0 \\
\hline no25* & -5050.0 & 100.00 & 55.061 & 5.0110 & 0 & -5050.0 & 100.00 & 55.061 & 5.0110 & 0 \\
\hline no260 & -5112.5 & 102.50 & 51.975 & 4.7785 & 0 & -5112.5 & 102.50 & 51.975 & 4.7785 & 0 \\
\hline no24* & -5125.0 & 93.000 & 48.995 & 5.6353 & 0 & -5125.0 & 93.000 & 48.995 & 5.6353 & 0 \\
\hline no302 & -5165.0 & 55.000 & 46.220 & 1.8172 & 0 & -5165.0 & 75.000 & 46.220 & 1.8172 & 0 \\
\hline no16* & -5240.0 & 50.000 & 46.316 & 4.9674 & 0 & -5240.0 & 75.000 & 46.316 & 4.9674 & 0 \\
\hline no263 & -5250.0 & 70.000 & 37.981 & 2.5234 & 0 & -5250.0 & 75.000 & 37.981 & 2.5234 & 0 \\
\hline no353 & -5266.0 & 50.000 & 40.501 & 2.6291 & 0 & -5266.0 & 75.000 & 40.501 & 2.6291 & 0 \\
\hline no23* & -5280.0 & 50.000 & 41.010 & 4.9782 & 0 & -5280.0 & 75.000 & 41.010 & 4.9782 & 0 \\
\hline no05* & -5398.0 & 72.000 & 32.751 & 4.9728 & 0 & -5398.0 & 75.000 & 32.751 & 4.9728 & 0 \\
\hline no326 & -5460.0 & 60.000 & 35.013 & 1.0875 & 0 & -5460.0 & 75.000 & 35.013 & 1.0875 & 0 \\
\hline no06* & -5470.0 & 50.000 & 36.181 & 5.5019 & 0 & -5470.0 & 75.000 & 36.181 & 5.5019 & 0 \\
\hline no262 & -5480.0 & 50.000 & 38.964 & 1.0034 & 0 & -5480.0 & 75.000 & 38.964 & 1.0034 & 0 \\
\hline no14* & -5485.0 & 50.000 & 46.557 & 5.0332 & 0 & -5485.0 & 75.000 & 46.557 & 5.0332 & 0 \\
\hline no259* & -5550.0 & 50.000 & 52.545 & 5.0110 & 0 & -5550.0 & 75.000 & 52.545 & 5.0110 & 0 \\
\hline no08* & -5557.5 & 67.500 & 41.824 & 5.0500 & 0 & -5557.5 & 75.000 & 41.824 & 5.0500 & 0 \\
\hline no07* & -5679.5 & 62.500 & 48.737 & 5.0110 & 0 & -5679.5 & 75.000 & 48.737 & 5.0110 & 0 \\
\hline no324 & -5700.0 & 100.00 & 44.234 & 4.9819 & 0 & -5700.0 & 100.00 & 44.234 & 4.9819 & 0 \\
\hline no261* & -5724.0 & 155.00 & 38.195 & 5.0670 & 0 & -5724.0 & 155.00 & 38.195 & 5.0670 & 0 \\
\hline no04* & -5730.0 & 100.00 & 44.880 & 5.0000 & 0 & -5730.0 & 100.00 & 44.880 & 5.0000 & 0 \\
\hline no241 & -5735.0 & 50.000 & 45.270 & 2.0000 & 0 & -5735.0 & 75.000 & 45.270 & 2.0000 & 0 \\
\hline no02* & -5740.0 & 50.000 & 52.766 & 5.0388 & 0 & -5740.0 & 75.000 & 52.766 & 5.0388 & 0 \\
\hline no139 & -5750.0 & 100.00 & 43.693 & 4.0581 & 0 & -5750.0 & 100.00 & 43.693 & 4.0581 & 0 \\
\hline no242* & -5759.5 & 50.000 & 62.000 & 5.0000 & 0 & -5759.5 & 75.000 & 62.000 & 5.0000 & 0 \\
\hline no243 & -5776.5 & 59.500 & 55.912 & 2.0985 & 0 & -5776.5 & 75.000 & 55.912 & 2.0985 & 0 \\
\hline no246 & -5790.0 & 80.000 & 56.779 & 4.5399 & 0 & -5790.0 & 80.000 & 56.779 & 4.5399 & 0 \\
\hline no247 & -5947.5 & 51.500 & 54.440 & 2.4929 & 0 & -5947.5 & 75.000 & 54.440 & 2.4929 & 0 \\
\hline
\end{tabular}




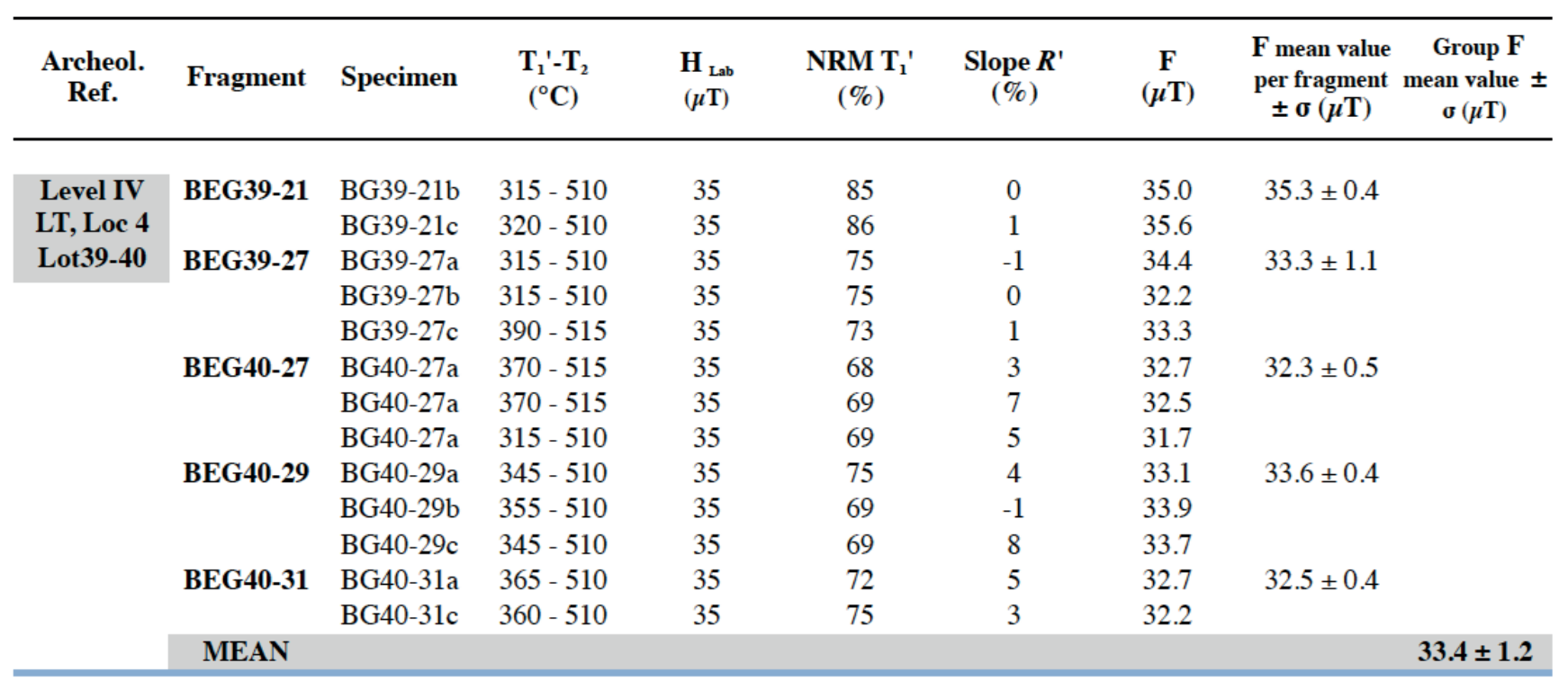




\section{Table S3}

\begin{tabular}{|c|c|c|c|c|c|c|}
\hline & & & & & & \\
\hline & & & & (14i/yi) & uгut uppei & \\
\hline-6000.000 & 48.375 & 68.527 & 22.567 & -0.00110 & 4.2570 & -2.8730 \\
\hline-5995.985 & 48.875 & 66.694 & 26.083 & 0.00790 & 2.3180 & -1.7160 \\
\hline-5991.971 & 49.125 & 65.713 & 27.805 & 0.0162 & 1.6990 & -1.2820 \\
\hline-5987.956 & 49.375 & 65.002 & 29.186 & 0.0235 & 1.3730 & -1.0770 \\
\hline-5983.942 & 49.375 & 64.398 & 30.268 & 0.0297 & 1.1800 & -0.95400 \\
\hline-5979.927 & 49.625 & 63.838 & 31.213 & 0.0348 & 1.0540 & -0.87200 \\
\hline-5975.912 & 49.625 & 63.419 & 32.048 & 0.0387 & 0.94900 & -0.81400 \\
\hline-5971.898 & 49.875 & 63.048 & 32.686 & 0.0416 & 0.87600 & -0.76600 \\
\hline-5967.883 & 49.875 & 62.763 & 33.255 & 0.0433 & 0.81500 & -0.73500 \\
\hline-5963.869 & 49.875 & 62.524 & 33.672 & 0.0440 & 0.77200 & -0.71100 \\
\hline-5959.854 & 50.125 & 62.400 & 33.973 & 0.0435 & 0.72900 & -0.69400 \\
\hline-5955.839 & 50.125 & 62.323 & 34.010 & 0.0420 & 0.69500 & -0.67800 \\
\hline-5951.825 & 50.125 & 62.162 & 34.003 & 0.0393 & 0.67400 & -0.67300 \\
\hline-5947.810 & 50.375 & 62.084 & 33.823 & 0.0355 & 0.65300 & -0.67300 \\
\hline-5943.796 & 50.375 & 62.050 & 33.497 & 0.0306 & 0.63900 & -0.67300 \\
\hline-5939.781 & 50.625 & 62.038 & 33.201 & 0.0247 & 0.62200 & -0.68100 \\
\hline-5935.766 & 50.625 & 61.994 & 32.811 & 0.0176 & 0.61200 & -0.68700 \\
\hline-5931.752 & 50.625 & 62.016 & 32.406 & 0.00940 & 0.60400 & -0.70500 \\
\hline-5927.737 & 50.875 & 62.077 & 32.071 & 0.000400 & 0.60100 & -0.71000 \\
\hline-5923.723 & 50.875 & 62.139 & 31.731 & -0.00800 & 0.58800 & -0.72300 \\
\hline-5919.708 & 50.875 & 62.262 & 31.407 & -0.0144 & 0.58000 & -0.73300 \\
\hline-5915.693 & 51.125 & 62.361 & 31.098 & -0.0184 & 0.57600 & -0.75300 \\
\hline-5911.679 & 51.125 & 62.539 & 30.790 & -0.0202 & 0.56600 & -0.76400 \\
\hline-5907.664 & 51.125 & 62.770 & 30.498 & -0.0195 & 0.56700 & -0.77400 \\
\hline-5903.650 & 51.125 & 63.052 & 30.216 & -0.0166 & 0.57400 & -0.77400 \\
\hline-5899.635 & 51.125 & 63.373 & 30.025 & -0.0113 & 0.57200 & -0.78800 \\
\hline-5895.620 & 51.125 & 63.740 & 29.946 & -0.00380 & 0.58200 & -0.78500 \\
\hline-5891.606 & 50.875 & 64.113 & 29.814 & 0.00620 & 0.59500 & -0.78200 \\
\hline-5887.591 & 50.875 & 64.484 & 29.692 & 0.0184 & 0.61800 & -0.77900 \\
\hline-5883.577 & 50.875 & 64.881 & 29.609 & 0.0330 & 0.65200 & -0.75800 \\
\hline-5879.562 & 50.875 & 65.179 & 29.553 & 0.0499 & 0.68700 & -0.74800 \\
\hline-5875.547 & 50.875 & 65.496 & 29.590 & 0.0691 & 0.73100 & -0.72900 \\
\hline-5871.533 & 51.125 & 65.853 & 29.634 & 0.0906 & 0.77200 & -0.70500 \\
\hline-5867.518 & 51.625 & 66.159 & 29.740 & 0.115 & 0.82300 & -0.68300 \\
\hline-5863.504 & 52.125 & 66.512 & 29.943 & 0.141 & 0.87200 & -0.66800 \\
\hline-5859.489 & 52.625 & 66.820 & 30.078 & 0.168 & 0.92000 & -0.64600 \\
\hline-5855.474 & 53.375 & 67.082 & 30.385 & 0.194 & 0.97100 & -0.61600 \\
\hline-5851.460 & 54.125 & 67.284 & 30.633 & 0.213 & 1.0190 & -0.57800 \\
\hline-5847.445 & 55.125 & 67.442 & 30.930 & 0.226 & 1.0790 & -0.55100 \\
\hline-5843.431 & 56.125 & 67.538 & 31.352 & 0.231 & 1.1420 & -0.51500 \\
\hline-5839.416 & 57.125 & 67.635 & 31.791 & 0.230 & 1.1960 & -0.48300 \\
\hline-5835.401 & 57.875 & 67.652 & 32.296 & 0.222 & 1.2570 & -0.44800 \\
\hline-5831.387 & 58.875 & 67.663 & 33.095 & 0.207 & 1.3010 & -0.40700 \\
\hline-5827.372 & 59.875 & 67.647 & 34.101 & 0.185 & 1.3460 & -0.36500 \\
\hline-5823.358 & 60.875 & 67.613 & 35.272 & 0.157 & 1.3510 & -0.32300 \\
\hline-5819.343 & 61.625 & 67.570 & 36.569 & 0.125 & 1.3650 & -0.28900 \\
\hline-5815.328 & 62.125 & 67.490 & 38.093 & 0.0925 & 1.3480 & -0.27900 \\
\hline
\end{tabular}




\begin{tabular}{|c|c|c|c|c|c|c|}
\hline-5811.314 & 62.125 & 67.386 & 39.919 & 0.0598 & 1.3150 & -0.28600 \\
\hline-5807.299 & 62.375 & 67.253 & 42.042 & 0.0270 & 1.2570 & -0.29900 \\
\hline-5803.285 & 62.125 & 67.114 & 44.064 & -0.00600 & 1.1550 & -0.31600 \\
\hline-5799.270 & 62.125 & 66.911 & 46.454 & -0.0392 & 1.0330 & -0.33800 \\
\hline-5795.255 & 61.875 & 66.693 & 49.260 & -0.0726 & 0.91000 & -0.36200 \\
\hline-5791.241 & 61.625 & 66.468 & 51.835 & -0.106 & 0.79700 & -0.39700 \\
\hline-5787.226 & 60.875 & 66.238 & 53.855 & -0.138 & 0.67100 & -0.43800 \\
\hline-5783.212 & 60.375 & 65.951 & 55.018 & -0.166 & 0.56300 & -0.49200 \\
\hline-5779.197 & 59.625 & 65.677 & 54.933 & -0.191 & 0.45600 & -0.54700 \\
\hline-5775.182 & 58.625 & 65.373 & 54.210 & -0.212 & 0.38000 & -0.60300 \\
\hline-5771.168 & 57.625 & 65.033 & 53.174 & -0.230 & 0.31700 & -0.66500 \\
\hline-5767.153 & 56.625 & 64.606 & 51.768 & -0.245 & 0.25700 & -0.73400 \\
\hline-5763.139 & 55.625 & 64.108 & 50.003 & -0.256 & 0.20600 & -0.78700 \\
\hline-5759.124 & 54.625 & 63.482 & 47.725 & -0.263 & 0.16500 & -0.83800 \\
\hline-5755.109 & 53.625 & 62.540 & 45.374 & -0.267 & 0.14800 & -0.88600 \\
\hline-5751.095 & 52.625 & 60.540 & 43.882 & -0.268 & 0.14800 & -0.93200 \\
\hline-5747.080 & 51.625 & 57.673 & 43.058 & -0.265 & 0.18800 & -0.96200 \\
\hline-5743.066 & 50.375 & 54.925 & 42.988 & -0.259 & 0.26400 & -0.96700 \\
\hline-5739.051 & 49.375 & 53.122 & 43.305 & -0.249 & 0.39400 & -0.91600 \\
\hline-5735.036 & 48.375 & 52.090 & 42.984 & -0.235 & 0.56000 & -0.81300 \\
\hline-5731.022 & 47.625 & 51.678 & 41.887 & -0.219 & 0.71300 & -0.69700 \\
\hline-5727.007 & 46.875 & 52.298 & 40.293 & -0.198 & 0.76700 & -0.60900 \\
\hline-5722.993 & 46.125 & 54.378 & 38.936 & -0.175 & 0.75400 & -0.55400 \\
\hline-5718.978 & 45.625 & 55.759 & 38.010 & -0.152 & 0.71300 & -0.51600 \\
\hline-5714.964 & 45.125 & 56.430 & 37.189 & -0.131 & 0.69700 & -0.48900 \\
\hline-5710.949 & 44.625 & 56.725 & 36.522 & -0.113 & 0.67900 & -0.47700 \\
\hline-5706.934 & 44.125 & 56.847 & 35.867 & -0.0983 & 0.69500 & -0.47200 \\
\hline-5702.920 & 43.875 & 56.908 & 35.316 & -0.0869 & 0.69000 & -0.48200 \\
\hline-5698.905 & 43.375 & 56.882 & 34.759 & -0.0785 & 0.68300 & -0.49600 \\
\hline-5694.891 & 43.125 & 56.713 & 34.185 & -0.0733 & 0.66500 & -0.51900 \\
\hline-5690.876 & 42.625 & 56.623 & 33.563 & -0.0712 & 0.64200 & -0.54800 \\
\hline-5686.861 & 42.375 & 56.666 & 32.975 & -0.0718 & 0.61400 & -0.57500 \\
\hline-5682.847 & 41.875 & 56.526 & 32.459 & -0.0731 & 0.58500 & -0.60700 \\
\hline-5678.832 & 41.625 & 56.409 & 32.088 & -0.0730 & 0.55500 & -0.62900 \\
\hline-5674.818 & 41.375 & 56.214 & 31.720 & -0.0712 & 0.53200 & -0.64900 \\
\hline-5670.803 & 41.125 & 55.845 & 31.437 & -0.0675 & 0.51100 & -0.67200 \\
\hline-5666.788 & 40.875 & 55.222 & 31.235 & -0.0622 & 0.51100 & -0.68900 \\
\hline-5662.774 & 40.625 & 54.445 & 31.044 & -0.0550 & 0.52500 & -0.70100 \\
\hline-5658.759 & 40.375 & 53.754 & 30.863 & -0.0460 & 0.54800 & -0.69900 \\
\hline-5654.745 & 40.375 & 53.382 & 30.708 & -0.0353 & 0.57400 & -0.69800 \\
\hline-5650.730 & 40.375 & 53.047 & 30.595 & -0.0225 & 0.59900 & -0.69700 \\
\hline-5646.715 & 40.375 & 52.863 & 30.499 & -0.00750 & 0.61800 & -0.69400 \\
\hline-5642.701 & 40.375 & 52.777 & 30.400 & 0.00980 & 0.63900 & -0.69400 \\
\hline-5638.686 & 40.375 & 52.921 & 30.278 & 0.0294 & 0.66700 & -0.68500 \\
\hline-5634.672 & 40.625 & 53.057 & 30.233 & 0.0512 & 0.69000 & -0.67900 \\
\hline-5630.657 & 40.875 & 53.322 & 30.179 & 0.0754 & 0.71500 & -0.67600 \\
\hline-5626.642 & 41.125 & 53.701 & 30.174 & 0.102 & 0.74700 & -0.66800 \\
\hline-5622.628 & 41.375 & 54.177 & 30.291 & 0.131 & 0.78600 & -0.65700 \\
\hline-5618.613 & 41.875 & 54.858 & 30.449 & 0.161 & 0.83800 & -0.63900 \\
\hline-5614.599 & 42.375 & 55.690 & 30.722 & 0.189 & 0.88500 & -0.61600 \\
\hline-5610.584 & 43.375 & 56.620 & 31.074 & 0.212 & 0.92400 & -0.59000 \\
\hline
\end{tabular}




\begin{tabular}{|c|c|c|c|c|c|c|}
\hline-5606.569 & 44.125 & 57.445 & 31.355 & 0.229 & 0.97600 & -0.54600 \\
\hline-5602.555 & 45.125 & 58.178 & 31.806 & 0.241 & 1.0250 & -0.50700 \\
\hline-5598.540 & 46.375 & 58.675 & 32.296 & 0.246 & 1.0450 & -0.47500 \\
\hline-5594.526 & 47.375 & 59.145 & 32.820 & 0.245 & 1.0690 & -0.44600 \\
\hline-5590.511 & 48.375 & 59.561 & 33.491 & 0.238 & 1.0740 & -0.42600 \\
\hline-5586.496 & 49.375 & 60.034 & 34.150 & 0.225 & 1.0810 & -0.42200 \\
\hline-5582.482 & 50.375 & 60.403 & 35.090 & 0.208 & 1.0840 & -0.41800 \\
\hline-5578.467 & 51.125 & 60.726 & 36.301 & 0.187 & 1.0850 & -0.42100 \\
\hline-5574.453 & 51.875 & 61.047 & 37.600 & 0.162 & 1.0850 & -0.43800 \\
\hline-5570.438 & 52.375 & 61.338 & 39.006 & 0.134 & 1.0370 & -0.45800 \\
\hline-5566.423 & 52.875 & 61.494 & 40.402 & 0.103 & 0.96900 & -0.49200 \\
\hline-5562.409 & 53.125 & 61.561 & 41.365 & 0.0677 & 0.90600 & -0.51600 \\
\hline-5558.394 & 53.375 & 61.551 & 41.939 & 0.0293 & 0.82400 & -0.53300 \\
\hline-5554.380 & 53.375 & 61.422 & 41.692 & -0.0127 & 0.73200 & -0.56100 \\
\hline-5550.365 & 53.375 & 61.226 & 41.019 & -0.0574 & 0.65000 & -0.57200 \\
\hline-5546.350 & 53.125 & 60.986 & 40.352 & -0.101 & 0.56400 & -0.58100 \\
\hline-5542.336 & 52.625 & 60.712 & 39.830 & -0.140 & 0.48700 & -0.58700 \\
\hline-5538.321 & 51.875 & 60.349 & 39.372 & -0.172 & 0.43700 & -0.60400 \\
\hline-5534.307 & 50.875 & 59.767 & 39.346 & -0.199 & 0.40000 & -0.61900 \\
\hline-5530.292 & 50.125 & 58.972 & 39.627 & -0.220 & 0.37000 & -0.64400 \\
\hline-5526.277 & 49.125 & 58.031 & 39.995 & -0.236 & 0.34300 & -0.66300 \\
\hline-5522.263 & 48.125 & 56.889 & 40.251 & -0.246 & 0.31200 & -0.67000 \\
\hline-5518.248 & 46.875 & 55.649 & 40.518 & -0.250 & 0.28600 & -0.65600 \\
\hline-5514.234 & 45.875 & 54.265 & 40.335 & -0.251 & 0.24800 & -0.64400 \\
\hline-5510.219 & 45.125 & 52.809 & 39.703 & -0.252 & 0.21400 & -0.63000 \\
\hline-5506.204 & 44.125 & 51.367 & 38.766 & -0.252 & 0.17900 & -0.61000 \\
\hline-5502.190 & 43.125 & 49.995 & 37.722 & -0.251 & 0.12400 & -0.58900 \\
\hline-5498.175 & 42.125 & 48.656 & 36.479 & -0.250 & 0.024000 & -0.57000 \\
\hline-5494.161 & 41.125 & 47.438 & 35.244 & -0.249 & 0.0020000 & -0.55200 \\
\hline-5490.146 & 40.375 & 46.322 & 34.129 & -0.247 & -0.018000 & -0.54300 \\
\hline-5486.131 & 39.375 & 45.310 & 32.922 & -0.244 & 0.019000 & -0.52900 \\
\hline-5482.117 & 38.375 & 44.309 & 31.576 & -0.242 & 0.048000 & -0.51400 \\
\hline-5478.102 & 37.625 & 43.271 & 30.355 & -0.237 & 0.053000 & -0.50300 \\
\hline-5474.088 & 36.625 & 42.148 & 29.670 & -0.230 & 0.057000 & -0.49400 \\
\hline-5470.073 & 35.875 & 40.989 & 29.215 & -0.220 & 0.059000 & -0.48500 \\
\hline-5466.058 & 34.875 & 39.752 & 28.815 & -0.207 & 0.062000 & -0.46900 \\
\hline-5462.044 & 34.125 & 38.582 & 28.450 & -0.192 & 0.066000 & -0.45300 \\
\hline-5458.029 & 33.125 & 37.412 & 28.183 & -0.174 & 0.069000 & -0.43600 \\
\hline-5454.015 & 32.375 & 36.441 & 27.930 & -0.153 & 0.074000 & -0.41400 \\
\hline-5450.000 & 31.625 & 35.623 & 27.712 & -0.129 & 0.080000 & -0.38800 \\
\hline-5445.985 & 30.875 & 34.887 & 27.524 & -0.105 & 0.089000 & -0.35500 \\
\hline-5441.971 & 30.625 & 34.193 & 27.394 & -0.0826 & 0.099000 & -0.32200 \\
\hline-5437.956 & 30.375 & 33.586 & 27.326 & -0.0612 & 0.11000 & -0.29400 \\
\hline-5433.942 & 30.125 & 33.055 & 27.269 & -0.0414 & 0.12100 & -0.26700 \\
\hline-5429.927 & 30.125 & 32.724 & 27.231 & -0.0230 & 0.13200 & -0.24300 \\
\hline-5425.912 & 30.125 & 32.668 & 27.226 & -0.00610 & 0.14200 & -0.22100 \\
\hline-5421.898 & 30.375 & 32.773 & 27.231 & 0.00920 & 0.15200 & -0.20000 \\
\hline-5417.883 & 30.375 & 32.974 & 27.260 & 0.0231 & 0.16200 & -0.18100 \\
\hline-5413.869 & 30.625 & 33.229 & 27.367 & 0.0355 & 0.17100 & -0.16400 \\
\hline-5409.854 & 30.875 & 33.515 & 27.512 & 0.0463 & 0.18000 & -0.14400 \\
\hline-5405.839 & 31.125 & 33.833 & 27.730 & 0.0557 & 0.18900 & -0.12100 \\
\hline
\end{tabular}




\begin{tabular}{|c|c|c|c|c|c|c|}
\hline Date & & & & & & \\
\hline & & & & & & \\
\hline-7000.000 & 54.875 & 68.604 & 22.238 & 0.0172 & 1.9990 & -1.0300 \\
\hline-6995.992 & 54.875 & 67.926 & 24.027 & 0.0165 & 1.4130 & -0.81200 \\
\hline-6991.984 & 54.875 & 67.578 & 25.073 & 0.0158 & 1.1230 & -0.69200 \\
\hline-6987.976 & 54.875 & 67.338 & 25.853 & 0.0151 & 0.94700 & -0.60200 \\
\hline-6983.968 & 55.125 & 67.130 & 26.494 & 0.0144 & 0.83300 & -0.53600 \\
\hline-6979.959 & 55.125 & 66.941 & 27.019 & 0.0138 & 0.74900 & -0.48800 \\
\hline-6975.951 & 55.125 & 66.778 & 27.489 & 0.0131 & 0.68400 & -0.45000 \\
\hline-6971.943 & 55.125 & 66.617 & 27.914 & 0.0124 & 0.63000 & -0.41600 \\
\hline-6967.935 & 55.125 & 66.482 & 28.272 & 0.0118 & 0.58600 & -0.38800 \\
\hline-6963.927 & 55.375 & 66.346 & 28.648 & 0.0111 & 0.54800 & -0.36400 \\
\hline-6959.919 & 55.375 & 66.212 & 28.923 & 0.0105 & 0.51600 & -0.34700 \\
\hline-6955.911 & 55.375 & 66.099 & 29.165 & 0.00980 & 0.49000 & -0.33100 \\
\hline-6951.903 & 55.375 & 65.975 & 29.399 & 0.00920 & 0.46700 & -0.31700 \\
\hline-6947.894 & 55.375 & 65.841 & 29.584 & 0.00860 & 0.44800 & -0.30300 \\
\hline-6943.886 & 55.375 & 65.727 & 29.792 & 0.00790 & 0.43200 & -0.29000 \\
\hline-6939.878 & 55.625 & 65.621 & 30.054 & 0.00730 & 0.41900 & -0.28100 \\
\hline-6935.870 & 55.625 & 65.501 & 30.292 & 0.00670 & 0.40500 & -0.27000 \\
\hline-6931.862 & 55.625 & 65.384 & 30.485 & 0.00610 & 0.39000 & -0.26300 \\
\hline-6927.854 & 55.625 & 65.272 & 30.727 & 0.00550 & 0.37900 & -0.25500 \\
\hline-6923.846 & 55.625 & 65.172 & 30.894 & 0.00490 & 0.36700 & -0.24800 \\
\hline-6919.838 & 55.625 & 65.064 & 31.060 & 0.00430 & 0.35700 & -0.24000 \\
\hline-6915.830 & 55.625 & 64.946 & 31.208 & 0.00370 & 0.34700 & -0.23500 \\
\hline-6911.821 & 55.625 & 64.844 & 31.371 & 0.00310 & 0.34100 & -0.22900 \\
\hline-6907.813 & 55.625 & 64.738 & 31.585 & 0.00260 & 0.33400 & -0.22400 \\
\hline-6903.805 & 55.625 & 64.630 & 31.721 & 0.00200 & 0.32800 & -0.21800 \\
\hline-6899.797 & 55.625 & 64.543 & 31.912 & 0.00140 & 0.32000 & -0.21200 \\
\hline-6895.789 & 55.625 & 64.444 & 32.086 & 0.000900 & 0.31300 & -0.20900 \\
\hline-6891.781 & 55.625 & 64.343 & 32.301 & 0.000300 & 0.30700 & -0.20600 \\
\hline-6887.773 & 55.625 & 64.230 & 32.456 & -0.000200 & 0.30300 & -0.20000 \\
\hline-6883.765 & 55.625 & 64.140 & 32.684 & -0.000800 & 0.29800 & -0.19500 \\
\hline-6879.757 & 55.625 & 64.053 & 32.874 & -0.00130 & 0.29200 & -0.19100 \\
\hline-6875.748 & 55.625 & 63.960 & 33.089 & -0.00180 & 0.28700 & -0.18800 \\
\hline-6871.740 & 55.625 & 63.877 & 33.336 & -0.00240 & 0.28300 & -0.18400 \\
\hline-6867.732 & 55.625 & 63.796 & 33.481 & -0.00290 & 0.27900 & -0.18200 \\
\hline-6863.724 & 55.625 & 63.707 & 33.691 & -0.00340 & 0.27600 & -0.18000 \\
\hline-6859.716 & 55.625 & 63.614 & 33.905 & -0.00390 & 0.27200 & -0.17600 \\
\hline-6855.708 & 55.625 & 63.519 & 34.161 & -0.00440 & 0.27000 & -0.17100 \\
\hline-6851.700 & 55.625 & 63.428 & 34.435 & -0.00490 & 0.26700 & -0.16700 \\
\hline-6847.692 & 55.625 & 63.348 & 34.660 & -0.00540 & 0.26600 & -0.16400 \\
\hline-6843.683 & 55.625 & 63.266 & 34.905 & -0.00590 & 0.26200 & -0.16300 \\
\hline-6839.675 & 55.625 & 63.196 & 35.185 & -0.00640 & 0.26000 & -0.15900 \\
\hline-6835.667 & 55.625 & 63.105 & 35.441 & -0.00690 & 0.25900 & -0.15500 \\
\hline-6831.659 & 55.375 & 63.017 & 35.713 & -0.00730 & 0.25600 & -0.15200 \\
\hline-6827.651 & 55.375 & 62.921 & 36.083 & -0.00780 & 0.25200 & -0.14800 \\
\hline-6823.643 & 55.375 & 62.832 & 36.365 & -0.00830 & 0.25200 & -0.14500 \\
\hline-6819.635 & 55.375 & 62.726 & 36.683 & -0.00870 & 0.24900 & -0.14200 \\
\hline-6815.627 & 55.375 & 62.622 & 37.006 & -0.00920 & 0.24800 & -0.13900 \\
\hline-6811.619 & 55.375 & 62.532 & 37.400 & -0.00960 & 0.24600 & -0.13300 \\
\hline
\end{tabular}




\begin{tabular}{|c|c|c|c|c|c|c|}
\hline-6807.610 & 55.375 & 62.407 & 37.733 & -0.0101 & 0.24400 & -0.13000 \\
\hline-6803.602 & 55.125 & 62.312 & 38.091 & -0.0105 & 0.24100 & -0.12800 \\
\hline-6799.594 & 55.125 & 62.197 & 38.492 & -0.0109 & 0.23700 & -0.12500 \\
\hline-6795.586 & 55.125 & 62.080 & 38.943 & -0.0114 & 0.23200 & -0.12300 \\
\hline-6791.578 & 55.125 & 61.953 & 39.341 & -0.0118 & 0.22800 & -0.12000 \\
\hline-6787.570 & 55.125 & 61.837 & 39.840 & -0.0122 & 0.22400 & -0.11700 \\
\hline-6783.562 & 54.875 & 61.694 & 40.341 & -0.0126 & 0.21900 & -0.11400 \\
\hline-6779.554 & 54.875 & 61.558 & 40.826 & -0.0130 & 0.21200 & -0.11300 \\
\hline-6775.545 & 54.875 & 61.426 & 41.255 & -0.0134 & 0.20600 & -0.11100 \\
\hline-6771.537 & 54.875 & 61.288 & 41.797 & -0.0138 & 0.20300 & -0.10900 \\
\hline-6767.529 & 54.875 & 61.135 & 42.304 & -0.0142 & 0.19900 & -0.10800 \\
\hline-6763.521 & 54.625 & 60.992 & 42.890 & -0.0146 & 0.19100 & -0.10700 \\
\hline-6759.513 & 54.625 & 60.830 & 43.467 & -0.0149 & 0.18300 & -0.10600 \\
\hline-6755.505 & 54.625 & 60.667 & 43.991 & -0.0153 & 0.17700 & -0.10500 \\
\hline-6751.497 & 54.625 & 60.504 & 44.521 & -0.0157 & 0.17300 & -0.10500 \\
\hline-6747.489 & 54.375 & 60.335 & 45.083 & -0.0160 & 0.16600 & -0.10400 \\
\hline-6743.481 & 54.375 & 60.165 & 45.575 & -0.0164 & 0.15900 & -0.10300 \\
\hline-6739.472 & 54.375 & 59.965 & 46.068 & -0.0167 & 0.15300 & -0.10300 \\
\hline-6735.464 & 54.375 & 59.774 & 46.568 & -0.0171 & 0.14700 & -0.10200 \\
\hline-6731.456 & 54.125 & 59.566 & 47.066 & -0.0174 & 0.14000 & -0.10100 \\
\hline-6727.448 & 54.125 & 59.387 & 47.493 & -0.0177 & 0.13400 & -0.10000 \\
\hline-6723.440 & 54.125 & 59.197 & 47.853 & -0.0181 & 0.12800 & -0.10000 \\
\hline-6719.432 & 53.875 & 59.014 & 48.188 & -0.0184 & 0.12100 & -0.10000 \\
\hline-6715.424 & 53.875 & 58.825 & 48.431 & -0.0187 & 0.11600 & -0.099000 \\
\hline-6711.416 & 53.875 & 58.644 & 48.577 & -0.0190 & 0.11100 & -0.10000 \\
\hline-6707.408 & 53.875 & 58.480 & 48.699 & -0.0193 & 0.10600 & -0.10000 \\
\hline-6703.399 & 53.625 & 58.322 & 48.790 & -0.0196 & 0.10100 & -0.10000 \\
\hline-6699.391 & 53.625 & 58.165 & 48.878 & -0.0199 & 0.097000 & -0.10000 \\
\hline-6695.383 & 53.625 & 58.027 & 48.920 & -0.0202 & 0.092000 & -0.099000 \\
\hline-6691.375 & 53.375 & 57.903 & 48.932 & -0.0205 & 0.087000 & -0.099000 \\
\hline-6687.367 & 53.375 & 57.793 & 48.919 & -0.0208 & 0.083000 & -0.099000 \\
\hline-6683.359 & 53.375 & 57.688 & 48.891 & -0.0210 & 0.080000 & -0.098000 \\
\hline-6679.351 & 53.125 & 57.580 & 48.839 & -0.0213 & 0.076000 & -0.098000 \\
\hline-6675.343 & 53.125 & 57.484 & 48.778 & -0.0216 & 0.073000 & -0.098000 \\
\hline-6671.334 & 53.125 & 57.391 & 48.697 & -0.0218 & 0.070000 & -0.098000 \\
\hline-6667.326 & 52.875 & 57.298 & 48.615 & -0.0221 & 0.068000 & -0.097000 \\
\hline-6663.318 & 52.875 & 57.228 & 48.532 & -0.0223 & 0.066000 & -0.097000 \\
\hline-6659.310 & 52.625 & 57.171 & 48.415 & -0.0226 & 0.064000 & -0.097000 \\
\hline-6655.302 & 52.625 & 57.106 & 48.303 & -0.0228 & 0.063000 & -0.097000 \\
\hline-6651.294 & 52.625 & 57.057 & 48.177 & -0.0230 & 0.062000 & -0.097000 \\
\hline-6647.286 & 52.375 & 57.029 & 48.038 & -0.0232 & 0.061000 & -0.097000 \\
\hline-6643.278 & 52.375 & 57.006 & 47.885 & -0.0235 & 0.061000 & -0.097000 \\
\hline-6639.270 & 52.375 & 57.002 & 47.744 & -0.0237 & 0.061000 & -0.097000 \\
\hline-6635.261 & 52.125 & 56.992 & 47.577 & -0.0239 & 0.060000 & -0.097000 \\
\hline-6631.253 & 52.125 & 56.992 & 47.413 & -0.0241 & 0.060000 & -0.097000 \\
\hline-6627.245 & 51.875 & 57.002 & 47.219 & -0.0243 & 0.061000 & -0.097000 \\
\hline-6623.237 & 51.875 & 57.018 & 47.014 & -0.0245 & 0.061000 & -0.097000 \\
\hline-6619.229 & 51.875 & 57.045 & 46.825 & -0.0247 & 0.061000 & -0.097000 \\
\hline-6615.221 & 51.625 & 57.076 & 46.644 & -0.0248 & 0.062000 & -0.097000 \\
\hline-6611.213 & 51.625 & 57.098 & 46.429 & -0.0250 & 0.063000 & -0.097000 \\
\hline-6607.205 & 51.375 & 57.129 & 46.205 & -0.0252 & 0.064000 & -0.097000 \\
\hline
\end{tabular}




\begin{tabular}{|c|c|c|c|c|c|c|}
\hline-6603.196 & 51.375 & 57.146 & 45.992 & -0.0253 & 0.065000 & -0.098000 \\
\hline-6599.188 & 51.375 & 57.198 & 45.803 & -0.0255 & 0.066000 & -0.099000 \\
\hline-6595.180 & 51.125 & 57.215 & 45.597 & -0.0257 & 0.066000 & -0.10000 \\
\hline-6591.172 & 51.125 & 57.251 & 45.372 & -0.0258 & 0.067000 & -0.10100 \\
\hline-6587.164 & 50.875 & 57.297 & 45.171 & -0.0259 & 0.069000 & -0.10200 \\
\hline-6583.156 & 50.875 & 57.302 & 44.955 & -0.0261 & 0.070000 & -0.10300 \\
\hline-6579.148 & 50.875 & 57.328 & 44.740 & -0.0262 & 0.072000 & -0.10500 \\
\hline-6575.140 & 50.625 & 57.327 & 44.535 & -0.0263 & 0.074000 & -0.10600 \\
\hline-6571.132 & 50.625 & 57.334 & 44.330 & -0.0265 & 0.075000 & -0.10700 \\
\hline-6567.123 & 50.375 & 57.325 & 44.110 & -0.0266 & 0.077000 & -0.10800 \\
\hline-6563.115 & 50.375 & 57.360 & 43.865 & -0.0267 & 0.079000 & -0.11000 \\
\hline-6559.107 & 50.375 & 57.406 & 43.679 & -0.0268 & 0.081000 & -0.11100 \\
\hline-6555.099 & 50.125 & 57.425 & 43.461 & -0.0269 & 0.082000 & -0.11300 \\
\hline-6551.091 & 50.125 & 57.486 & 43.235 & -0.0270 & 0.083000 & -0.11600 \\
\hline-6547.083 & 49.875 & 57.487 & 43.007 & -0.0271 & 0.084000 & -0.11800 \\
\hline-6543.075 & 49.875 & 57.493 & 42.806 & -0.0272 & 0.085000 & -0.12100 \\
\hline-6539.067 & 49.625 & 57.485 & 42.566 & -0.0272 & 0.087000 & -0.12200 \\
\hline-6535.059 & 49.625 & 57.500 & 42.318 & -0.0273 & 0.089000 & -0.12300 \\
\hline-6531.050 & 49.625 & 57.495 & 42.086 & -0.0274 & 0.090000 & -0.12500 \\
\hline-6527.042 & 49.375 & 57.486 & 41.883 & -0.0274 & 0.090000 & -0.12700 \\
\hline-6523.034 & 49.375 & 57.471 & 41.656 & -0.0275 & 0.091000 & -0.12900 \\
\hline-6519.026 & 49.125 & 57.470 & 41.470 & -0.0275 & 0.092000 & -0.13200 \\
\hline-6515.018 & 49.125 & 57.477 & 41.247 & -0.0276 & 0.094000 & -0.13400 \\
\hline-6511.010 & 48.875 & 57.477 & 41.055 & -0.0276 & 0.094000 & -0.13800 \\
\hline-6507.002 & 48.875 & 57.475 & 40.888 & -0.0277 & 0.096000 & -0.14000 \\
\hline-6502.994 & 48.625 & 57.444 & 40.718 & -0.0277 & 0.097000 & -0.14200 \\
\hline-6498.985 & 48.625 & 57.419 & 40.579 & -0.0277 & 0.098000 & -0.14500 \\
\hline-6494.977 & 48.625 & 57.399 & 40.451 & -0.0277 & 0.099000 & -0.14700 \\
\hline-6490.969 & 48.375 & 57.383 & 40.286 & -0.0278 & 0.099000 & -0.15000 \\
\hline-6486.961 & 48.375 & 57.356 & 40.178 & -0.0279 & 0.099000 & -0.15300 \\
\hline-6482.953 & 48.125 & 57.330 & 40.068 & -0.0279 & 0.099000 & -0.15600 \\
\hline-6478.945 & 48.125 & 57.284 & 39.969 & -0.0280 & 0.10000 & -0.15800 \\
\hline-6474.937 & 47.875 & 57.223 & 39.878 & -0.0282 & 0.10000 & -0.16100 \\
\hline-6470.929 & 47.875 & 57.174 & 39.763 & -0.0283 & 0.10000 & -0.16300 \\
\hline-6466.921 & 47.625 & 57.117 & 39.657 & -0.0285 & 0.099000 & -0.16500 \\
\hline-6462.912 & 47.625 & 57.055 & 39.548 & -0.0286 & 0.099000 & -0.16700 \\
\hline-6458.904 & 47.375 & 57.034 & 39.436 & -0.0288 & 0.099000 & -0.16900 \\
\hline-6454.896 & 47.375 & 56.953 & 39.345 & -0.0290 & 0.099000 & -0.17100 \\
\hline-6450.888 & 47.125 & 56.918 & 39.217 & -0.0293 & 0.10000 & -0.17200 \\
\hline-6446.880 & 47.125 & 56.870 & 39.111 & -0.0295 & 0.10100 & -0.17400 \\
\hline-6442.872 & 46.875 & 56.788 & 39.009 & -0.0298 & 0.10100 & -0.17600 \\
\hline-6438.864 & 46.875 & 56.699 & 38.922 & -0.0301 & 0.10000 & -0.17900 \\
\hline-6434.856 & 46.625 & 56.624 & 38.823 & -0.0304 & 0.098000 & -0.18300 \\
\hline-6430.847 & 46.625 & 56.522 & 38.741 & -0.0307 & 0.098000 & -0.18400 \\
\hline-6426.839 & 46.375 & 56.422 & 38.628 & -0.0310 & 0.097000 & -0.18600 \\
\hline-6422.831 & 46.375 & 56.278 & 38.518 & -0.0314 & 0.096000 & -0.18800 \\
\hline-6418.823 & 46.125 & 56.122 & 38.442 & -0.0317 & 0.095000 & -0.18900 \\
\hline-6414.815 & 46.125 & 56.001 & 38.412 & -0.0321 & 0.095000 & -0.19200 \\
\hline-6410.807 & 45.875 & 55.870 & 38.320 & -0.0326 & 0.094000 & -0.19400 \\
\hline-6406.799 & 45.875 & 55.752 & 38.234 & -0.0330 & 0.093000 & -0.19500 \\
\hline-6402.791 & 45.625 & 55.590 & 38.161 & -0.0334 & 0.093000 & -0.19600 \\
\hline
\end{tabular}




\begin{tabular}{|c|c|c|c|c|c|c|}
\hline-6398.783 & 45.625 & 55.429 & 38.082 & -0.0339 & 0.092000 & -0.19800 \\
\hline-6394.774 & 45.375 & 55.252 & 38.013 & -0.0344 & 0.091000 & -0.20000 \\
\hline-6390.766 & 45.375 & 55.097 & 37.965 & -0.0349 & 0.091000 & -0.20100 \\
\hline-6386.758 & 45.125 & 54.961 & 37.900 & -0.0354 & 0.089000 & -0.20200 \\
\hline-6382.750 & 45.125 & 54.800 & 37.851 & -0.0359 & 0.088000 & -0.20200 \\
\hline-6378.742 & 44.875 & 54.612 & 37.825 & -0.0365 & 0.086000 & -0.20400 \\
\hline-6374.734 & 44.875 & 54.400 & 37.764 & -0.0371 & 0.085000 & -0.20500 \\
\hline-6370.726 & 44.625 & 54.169 & 37.732 & -0.0376 & 0.085000 & -0.20700 \\
\hline-6366.718 & 44.625 & 53.960 & 37.692 & -0.0381 & 0.081000 & -0.21000 \\
\hline-6362.710 & 44.375 & 53.743 & 37.710 & -0.0384 & 0.080000 & -0.21100 \\
\hline-6358.701 & 44.125 & 53.479 & 37.735 & -0.0386 & 0.079000 & -0.21100 \\
\hline-6354.693 & 44.125 & 53.234 & 37.734 & -0.0385 & 0.077000 & -0.21300 \\
\hline-6350.685 & 43.875 & 52.999 & 37.733 & -0.0383 & 0.076000 & -0.21300 \\
\hline-6346.677 & 43.875 & 52.740 & 37.744 & -0.0379 & 0.074000 & -0.21300 \\
\hline-6342.669 & 43.625 & 52.473 & 37.771 & -0.0373 & 0.070000 & -0.21200 \\
\hline-6338.661 & 43.625 & 52.194 & 37.852 & -0.0365 & 0.069000 & -0.21200 \\
\hline-6334.653 & 43.375 & 51.926 & 37.898 & -0.0356 & 0.067000 & -0.21100 \\
\hline-6330.645 & 43.125 & 51.606 & 37.950 & -0.0344 & 0.064000 & -0.21000 \\
\hline-6326.636 & 43.125 & 51.321 & 38.002 & -0.0331 & 0.063000 & -0.20800 \\
\hline-6322.628 & 42.875 & 50.998 & 38.079 & -0.0316 & 0.061000 & -0.20500 \\
\hline-6318.620 & 42.875 & 50.708 & 38.147 & -0.0299 & 0.059000 & -0.20100 \\
\hline-6314.612 & 42.625 & 50.345 & 38.235 & -0.0280 & 0.057000 & -0.19500 \\
\hline-6310.604 & 42.625 & 50.018 & 38.336 & -0.0259 & 0.054000 & -0.19100 \\
\hline-6306.596 & 42.375 & 49.702 & 38.435 & -0.0237 & 0.051000 & -0.18500 \\
\hline-6302.588 & 42.375 & 49.357 & 38.513 & -0.0214 & 0.049000 & -0.17700 \\
\hline-6298.580 & 42.125 & 49.012 & 38.610 & -0.0193 & 0.047000 & -0.16800 \\
\hline-6294.572 & 42.125 & 48.653 & 38.706 & -0.0173 & 0.045000 & -0.15900 \\
\hline-6290.563 & 42.125 & 48.283 & 38.780 & -0.0154 & 0.043000 & -0.15000 \\
\hline-6286.555 & 41.875 & 47.970 & 38.858 & -0.0137 & 0.042000 & -0.14200 \\
\hline-6282.547 & 41.875 & 47.642 & 38.921 & -0.0121 & 0.039000 & -0.13400 \\
\hline-6278.539 & 41.875 & 47.345 & 38.975 & -0.0106 & 0.037000 & -0.12600 \\
\hline-6274.531 & 41.875 & 47.061 & 39.015 & -0.00930 & 0.035000 & -0.12000 \\
\hline-6270.523 & 41.875 & 46.784 & 39.084 & -0.00810 & 0.033000 & -0.11300 \\
\hline-6266.515 & 41.625 & 46.517 & 39.137 & -0.00700 & 0.031000 & -0.10600 \\
\hline-6262.507 & 41.625 & 46.282 & 39.183 & -0.00610 & 0.030000 & -0.10000 \\
\hline-6258.498 & 41.625 & 46.063 & 39.233 & -0.00530 & 0.028000 & -0.093000 \\
\hline-6254.490 & 41.625 & 45.859 & 39.281 & -0.00470 & 0.027000 & -0.088000 \\
\hline-6250.482 & 41.625 & 45.666 & 39.313 & -0.00410 & 0.026000 & -0.082000 \\
\hline-6246.474 & 41.625 & 45.502 & 39.353 & -0.00370 & 0.024000 & -0.077000 \\
\hline-6242.466 & 41.625 & 45.336 & 39.400 & -0.00340 & 0.024000 & -0.073000 \\
\hline-6238.458 & 41.625 & 45.179 & 39.436 & -0.00310 & 0.023000 & -0.068000 \\
\hline-6234.450 & 41.625 & 45.039 & 39.478 & -0.00280 & 0.022000 & -0.063000 \\
\hline-6230.442 & 41.625 & 44.899 & 39.505 & -0.00250 & 0.021000 & -0.059000 \\
\hline-6226.434 & 41.625 & 44.754 & 39.540 & -0.00230 & 0.021000 & -0.056000 \\
\hline-6222.425 & 41.625 & 44.629 & 39.574 & -0.00200 & 0.020000 & -0.052000 \\
\hline-6218.417 & 41.625 & 44.518 & 39.615 & -0.00170 & 0.020000 & -0.049000 \\
\hline-6214.409 & 41.625 & 44.401 & 39.650 & -0.00140 & 0.019000 & -0.046000 \\
\hline-6210.401 & 41.625 & 44.288 & 39.685 & -0.00120 & 0.018000 & -0.043000 \\
\hline-6206.393 & 41.625 & 44.179 & 39.719 & -0.000900 & 0.018000 & -0.041000 \\
\hline-6202.385 & 41.625 & 44.074 & 39.756 & -0.000600 & 0.017000 & -0.039000 \\
\hline-6198.377 & 41.625 & 43.982 & 39.783 & -0.000400 & 0.017000 & -0.037000 \\
\hline
\end{tabular}




\begin{tabular}{|c|c|c|c|c|c|c|}
\hline-6194.369 & 41.625 & 43.883 & 39.814 & -0.000200 & 0.017000 & -0.035000 \\
\hline-6190.361 & 41.625 & 43.797 & 39.845 & $1.00 \mathrm{e}-04$ & 0.016000 & -0.033000 \\
\hline-6186.352 & 41.625 & 43.711 & 39.884 & 0.000300 & 0.016000 & -0.032000 \\
\hline-6182.344 & 41.625 & 43.634 & 39.918 & 0.000500 & 0.016000 & -0.030000 \\
\hline-6178.336 & 41.625 & 43.554 & 39.949 & 0.000800 & 0.016000 & -0.029000 \\
\hline-6174.328 & 41.625 & 43.483 & 39.979 & 0.00100 & 0.015000 & -0.028000 \\
\hline-6170.320 & 41.625 & 43.423 & 40.014 & 0.00120 & 0.015000 & -0.027000 \\
\hline-6166.312 & 41.625 & 43.364 & 40.043 & 0.00140 & 0.015000 & -0.027000 \\
\hline-6162.304 & 41.625 & 43.310 & 40.077 & 0.00160 & 0.015000 & -0.026000 \\
\hline-6158.296 & 41.625 & 43.266 & 40.110 & 0.00180 & 0.015000 & -0.025000 \\
\hline-6154.287 & 41.625 & 43.225 & 40.143 & 0.00200 & 0.015000 & -0.024000 \\
\hline-6150.279 & 41.625 & 43.189 & 40.172 & 0.00220 & 0.014000 & -0.024000 \\
\hline-6146.271 & 41.625 & 43.156 & 40.202 & 0.00230 & 0.014000 & -0.023000 \\
\hline-6142.263 & 41.625 & 43.130 & 40.237 & 0.00250 & 0.014000 & -0.022000 \\
\hline-6138.255 & 41.625 & 43.106 & 40.268 & 0.00270 & 0.014000 & -0.022000 \\
\hline-6134.247 & 41.875 & 43.086 & 40.302 & 0.00280 & 0.014000 & -0.021000 \\
\hline-6130.239 & 41.875 & 43.067 & 40.334 & 0.00300 & 0.014000 & -0.020000 \\
\hline-6126.231 & 41.875 & 43.055 & 40.367 & 0.00310 & 0.014000 & -0.020000 \\
\hline-6122.223 & 41.875 & 43.043 & 40.400 & 0.00330 & 0.014000 & -0.019000 \\
\hline-6118.214 & 41.875 & 43.034 & 40.434 & 0.00340 & 0.014000 & -0.018000 \\
\hline-6114.206 & 41.875 & 43.025 & 40.464 & 0.00360 & 0.014000 & -0.018000 \\
\hline-6110.198 & 41.875 & 43.014 & 40.498 & 0.00370 & 0.014000 & -0.017000 \\
\hline-6106.190 & 41.875 & 43.007 & 40.532 & 0.00380 & 0.014000 & -0.016000 \\
\hline-6102.182 & 41.875 & 43.002 & 40.568 & 0.00390 & 0.014000 & -0.015000 \\
\hline-6098.174 & 41.875 & 42.997 & 40.601 & 0.00400 & 0.013000 & -0.014000 \\
\hline-6094.166 & 41.875 & 42.996 & 40.632 & 0.00410 & 0.013000 & -0.013000 \\
\hline-6090.158 & 41.875 & 42.993 & 40.666 & 0.00420 & 0.013000 & -0.012000 \\
\hline-6086.149 & 41.875 & 42.994 & 40.700 & 0.00430 & 0.013000 & -0.011000 \\
\hline-6082.141 & 41.875 & 42.995 & 40.734 & 0.00440 & 0.013000 & -0.0100000 \\
\hline-6078.133 & 41.875 & 42.996 & 40.766 & 0.00450 & 0.013000 & -0.0090000 \\
\hline-6074.125 & 41.875 & 42.998 & 40.799 & 0.00460 & 0.013000 & -0.0080000 \\
\hline-6070.117 & 42.125 & 43.001 & 40.833 & 0.00470 & 0.013000 & -0.0070000 \\
\hline-6066.109 & 42.125 & 43.005 & 40.865 & 0.00470 & 0.013000 & -0.0070000 \\
\hline-6062.101 & 42.125 & 43.009 & 40.898 & 0.00480 & 0.013000 & -0.0060000 \\
\hline-6058.093 & 42.125 & 43.013 & 40.933 & 0.00490 & 0.013000 & -0.0060000 \\
\hline-6054.085 & 42.125 & 43.018 & 40.964 & 0.00490 & 0.013000 & -0.0060000 \\
\hline-6050.076 & 42.125 & 43.025 & 40.997 & 0.00500 & 0.013000 & -0.0050000 \\
\hline-6046.068 & 42.125 & 43.032 & 41.030 & 0.00500 & 0.013000 & -0.0050000 \\
\hline-6042.060 & 42.125 & 43.039 & 41.063 & 0.00500 & 0.013000 & -0.0050000 \\
\hline-6038.052 & 42.125 & 43.045 & 41.095 & 0.00510 & 0.013000 & -0.0050000 \\
\hline-6034.044 & 42.125 & 43.054 & 41.128 & 0.00510 & 0.013000 & -0.0050000 \\
\hline-6030.036 & 42.125 & 43.061 & 41.158 & 0.00510 & 0.013000 & -0.0050000 \\
\hline-6026.028 & 42.125 & 43.071 & 41.189 & 0.00510 & 0.013000 & -0.0040000 \\
\hline-6022.020 & 42.125 & 43.080 & 41.220 & 0.00510 & 0.013000 & -0.0040000 \\
\hline-6018.012 & 42.125 & 43.089 & 41.249 & 0.00510 & 0.013000 & -0.0040000 \\
\hline-6014.003 & 42.125 & 43.099 & 41.280 & 0.00510 & 0.013000 & -0.0040000 \\
\hline-6009.995 & 42.375 & 43.110 & 41.309 & 0.00510 & 0.013000 & -0.0040000 \\
\hline-6005.987 & 42.375 & 43.121 & 41.339 & 0.00510 & 0.013000 & -0.0040000 \\
\hline-6001.979 & 42.375 & 43.134 & 41.367 & 0.00510 & 0.013000 & -0.0040000 \\
\hline-5997.971 & 42.375 & 43.148 & 41.395 & 0.00510 & 0.012000 & -0.0040000 \\
\hline-5993.963 & 42.375 & 43.162 & 41.424 & 0.00500 & 0.012000 & -0.0040000 \\
\hline
\end{tabular}




\begin{tabular}{|c|c|c|c|c|c|c|}
\hline-5989.955 & 42.375 & 43.177 & 41.451 & 0.00500 & 0.012000 & -0.0040000 \\
\hline-5985.947 & 42.375 & 43.190 & 41.477 & 0.00500 & 0.013000 & -0.0030000 \\
\hline-5981.938 & 42.375 & 43.205 & 41.502 & 0.00500 & 0.013000 & -0.0030000 \\
\hline-5977.930 & 42.375 & 43.223 & 41.527 & 0.00490 & 0.013000 & -0.0030000 \\
\hline-5973.922 & 42.375 & 43.239 & 41.551 & 0.00490 & 0.013000 & -0.0030000 \\
\hline-5969.914 & 42.375 & 43.259 & 41.575 & 0.00490 & 0.013000 & -0.0030000 \\
\hline-5965.906 & 42.375 & 43.279 & 41.600 & 0.00480 & 0.013000 & -0.0030000 \\
\hline-5961.898 & 42.375 & 43.302 & 41.622 & 0.00480 & 0.013000 & -0.0030000 \\
\hline-5957.890 & 42.375 & 43.324 & 41.645 & 0.00480 & 0.013000 & -0.0030000 \\
\hline-5953.882 & 42.625 & 43.351 & 41.667 & 0.00480 & 0.013000 & -0.0030000 \\
\hline-5949.874 & 42.625 & 43.379 & 41.687 & 0.00470 & 0.013000 & -0.0030000 \\
\hline-5945.865 & 42.625 & 43.408 & 41.708 & 0.00470 & 0.013000 & -0.0030000 \\
\hline-5941.857 & 42.625 & 43.436 & 41.728 & 0.00470 & 0.013000 & -0.0030000 \\
\hline-5937.849 & 42.625 & 43.467 & 41.745 & 0.00460 & 0.013000 & -0.0030000 \\
\hline-5933.841 & 42.625 & 43.504 & 41.762 & 0.00460 & 0.013000 & -0.0030000 \\
\hline-5929.833 & 42.625 & 43.541 & 41.779 & 0.00460 & 0.013000 & -0.0030000 \\
\hline-5925.825 & 42.625 & 43.581 & 41.797 & 0.00450 & 0.013000 & -0.0030000 \\
\hline-5921.817 & 42.625 & 43.619 & 41.810 & 0.00450 & 0.013000 & -0.0030000 \\
\hline-5917.809 & 42.625 & 43.663 & 41.822 & 0.00450 & 0.013000 & -0.0030000 \\
\hline-5913.801 & 42.625 & 43.709 & 41.833 & 0.00440 & 0.013000 & -0.0030000 \\
\hline-5909.792 & 42.625 & 43.752 & 41.846 & 0.00440 & 0.013000 & -0.0030000 \\
\hline-5905.784 & 42.625 & 43.800 & 41.858 & 0.00430 & 0.013000 & -0.0030000 \\
\hline-5901.776 & 42.625 & 43.845 & 41.870 & 0.00430 & 0.014000 & -0.0030000 \\
\hline-5897.768 & 42.625 & 43.891 & 41.880 & 0.00430 & 0.014000 & -0.0030000 \\
\hline-5893.760 & 42.875 & 43.939 & 41.889 & 0.00420 & 0.014000 & -0.0030000 \\
\hline-5889.752 & 42.875 & 43.988 & 41.899 & 0.00420 & 0.014000 & -0.0030000 \\
\hline-5885.744 & 42.875 & 44.034 & 41.910 & 0.00420 & 0.014000 & -0.0030000 \\
\hline-5881.736 & 42.875 & 44.085 & 41.921 & 0.00410 & 0.014000 & -0.0030000 \\
\hline-5877.727 & 42.875 & 44.131 & 41.929 & 0.00410 & 0.014000 & -0.0030000 \\
\hline-5873.719 & 42.875 & 44.175 & 41.936 & 0.00400 & 0.014000 & -0.0030000 \\
\hline-5869.711 & 42.875 & 44.224 & 41.944 & 0.00400 & 0.014000 & -0.0030000 \\
\hline-5865.703 & 42.875 & 44.273 & 41.960 & 0.00400 & 0.014000 & -0.0030000 \\
\hline-5861.695 & 42.875 & 44.319 & 41.976 & 0.00390 & 0.015000 & -0.0030000 \\
\hline-5857.687 & 42.875 & 44.369 & 41.992 & 0.00390 & 0.015000 & -0.0030000 \\
\hline-5853.679 & 42.875 & 44.416 & 42.012 & 0.00380 & 0.015000 & -0.0030000 \\
\hline-5849.671 & 42.875 & 44.465 & 42.039 & 0.00380 & 0.015000 & -0.0030000 \\
\hline-5845.663 & 42.875 & 44.516 & 42.060 & 0.00370 & 0.015000 & -0.0040000 \\
\hline-5841.654 & 42.875 & 44.565 & 42.091 & 0.00370 & 0.016000 & -0.0040000 \\
\hline-5837.646 & 43.125 & 44.611 & 42.127 & 0.00360 & 0.016000 & -0.0040000 \\
\hline-5833.638 & 43.125 & 44.664 & 42.164 & 0.00360 & 0.016000 & -0.0040000 \\
\hline-5829.630 & 43.125 & 44.712 & 42.205 & 0.00360 & 0.016000 & -0.0050000 \\
\hline-5825.622 & 43.125 & 44.763 & 42.249 & 0.00350 & 0.016000 & -0.0050000 \\
\hline-5821.614 & 43.125 & 44.810 & 42.304 & 0.00350 & 0.017000 & -0.0050000 \\
\hline-5817.606 & 43.125 & 44.857 & 42.350 & 0.00340 & 0.017000 & -0.0060000 \\
\hline-5813.598 & 43.125 & 44.898 & 42.400 & 0.00340 & 0.017000 & -0.0070000 \\
\hline-5809.589 & 43.125 & 44.935 & 42.445 & 0.00330 & 0.017000 & -0.0080000 \\
\hline-5805.581 & 43.125 & 44.970 & 42.491 & 0.00330 & 0.017000 & -0.0090000 \\
\hline-5801.573 & 43.125 & 45.003 & 42.535 & 0.00320 & 0.017000 & -0.0100000 \\
\hline-5797.565 & 43.125 & 45.023 & 42.578 & 0.00320 & 0.017000 & -0.011000 \\
\hline-5793.557 & 43.125 & 45.051 & 42.613 & 0.00310 & 0.017000 & -0.013000 \\
\hline-5789.549 & 43.125 & 45.055 & 42.647 & 0.00310 & 0.017000 & -0.014000 \\
\hline
\end{tabular}




\begin{tabular}{|c|c|c|c|c|c|c|}
\hline-5785.541 & 43.125 & 45.062 & 42.677 & 0.00300 & 0.017000 & -0.016000 \\
\hline-5781.533 & 43.375 & 45.053 & 42.703 & 0.00300 & 0.017000 & -0.018000 \\
\hline-5777.525 & 43.375 & 45.033 & 42.729 & 0.00290 & 0.017000 & -0.020000 \\
\hline-5773.516 & 43.375 & 44.998 & 42.754 & 0.00290 & 0.016000 & -0.022000 \\
\hline-5769.508 & 43.375 & 44.965 & 42.778 & 0.00280 & 0.016000 & -0.024000 \\
\hline-5765.500 & 43.375 & 44.928 & 42.800 & 0.00280 & 0.016000 & -0.026000 \\
\hline-5761.492 & 43.375 & 44.887 & 42.820 & 0.00270 & 0.016000 & -0.028000 \\
\hline-5757.484 & 43.375 & 44.851 & 42.838 & 0.00270 & 0.016000 & -0.031000 \\
\hline-5753.476 & 43.375 & 44.815 & 42.853 & 0.00260 & 0.016000 & -0.033000 \\
\hline-5749.468 & 43.375 & 44.781 & 42.867 & 0.00260 & 0.015000 & -0.036000 \\
\hline-5745.460 & 43.375 & 44.742 & 42.877 & 0.00250 & 0.015000 & -0.040000 \\
\hline-5741.452 & 43.375 & 44.698 & 42.884 & 0.00250 & 0.015000 & -0.044000 \\
\hline-5737.443 & 43.375 & 44.651 & 42.882 & 0.00260 & 0.015000 & -0.048000 \\
\hline-5733.435 & 43.375 & 44.604 & 42.874 & 0.00280 & 0.014000 & -0.052000 \\
\hline-5729.427 & 43.375 & 44.561 & 42.859 & 0.00300 & 0.014000 & -0.056000 \\
\hline-5725.419 & 43.375 & 44.526 & 42.823 & 0.00340 & 0.014000 & -0.060000 \\
\hline-5721.411 & 43.375 & 44.496 & 42.753 & 0.00390 & 0.014000 & -0.064000 \\
\hline-5717.403 & 43.375 & 44.471 & 42.633 & 0.00440 & 0.014000 & -0.068000 \\
\hline-5713.395 & 43.375 & 44.450 & 42.467 & 0.00510 & 0.013000 & -0.072000 \\
\hline-5709.387 & 43.625 & 44.440 & 42.268 & 0.00580 & 0.013000 & -0.077000 \\
\hline-5705.378 & 43.625 & 44.436 & 42.040 & 0.00670 & 0.013000 & -0.082000 \\
\hline-5701.370 & 43.625 & 44.435 & 41.793 & 0.00760 & 0.013000 & -0.088000 \\
\hline-5697.362 & 43.625 & 44.442 & 41.517 & 0.00860 & 0.013000 & -0.095000 \\
\hline-5693.354 & 43.625 & 44.450 & 41.233 & 0.00970 & 0.012000 & -0.10200 \\
\hline-5689.346 & 43.625 & 44.461 & 40.926 & 0.0109 & 0.012000 & -0.11000 \\
\hline-5685.338 & 43.625 & 44.473 & 40.597 & 0.0122 & 0.012000 & -0.11900 \\
\hline-5681.330 & 43.625 & 44.484 & 40.249 & 0.0135 & 0.012000 & -0.12900 \\
\hline-5677.322 & 43.625 & 44.499 & 39.884 & 0.0138 & 0.011000 & -0.14200 \\
\hline-5673.314 & 43.625 & 44.510 & 39.508 & 0.0124 & 0.011000 & -0.15500 \\
\hline-5669.305 & 43.625 & 44.522 & 39.102 & 0.00920 & 0.011000 & -0.17000 \\
\hline-5665.297 & 43.625 & 44.535 & 38.674 & 0.00410 & 0.011000 & -0.18800 \\
\hline-5661.289 & 43.625 & 44.538 & 38.229 & -0.00290 & 0.0100000 & -0.20700 \\
\hline-5657.281 & 43.625 & 44.545 & 37.725 & -0.0118 & 0.0100000 & -0.23200 \\
\hline-5653.273 & 43.375 & 44.553 & 37.180 & -0.0226 & 0.0100000 & -0.26000 \\
\hline-5649.265 & 43.375 & 44.559 & 36.541 & -0.0352 & 0.0100000 & -0.29000 \\
\hline-5645.257 & 43.375 & 44.561 & 35.814 & -0.0498 & 0.0100000 & -0.32300 \\
\hline-5641.249 & 43.375 & 44.555 & 35.008 & -0.0662 & 0.0100000 & -0.36300 \\
\hline-5637.240 & 43.375 & 44.550 & 34.049 & -0.0844 & 0.0100000 & -0.40800 \\
\hline-5633.232 & 43.125 & 44.541 & 32.969 & -0.105 & 0.0100000 & -0.45900 \\
\hline-5629.224 & 42.625 & 44.536 & 31.791 & -0.127 & 0.011000 & -0.51700 \\
\hline-5625.216 & 41.875 & 44.526 & 30.489 & -0.150 & 0.015000 & -0.58300 \\
\hline-5621.208 & 41.125 & 44.509 & 29.261 & -0.176 & 0.041000 & -0.65400 \\
\hline-5617.200 & 40.125 & 44.480 & 28.196 & -0.198 & 0.13200 & -0.72500 \\
\hline-5613.192 & 39.375 & 44.446 & 27.379 & -0.213 & 0.19900 & -0.80000 \\
\hline-5609.184 & 38.375 & 44.399 & 26.738 & -0.221 & 0.26600 & -0.87700 \\
\hline-5605.176 & 37.375 & 44.346 & 26.309 & -0.222 & 0.34100 & -0.94800 \\
\hline-5601.167 & 36.375 & 44.284 & 26.085 & -0.215 & 0.44600 & -1.0000 \\
\hline-5597.159 & 35.625 & 44.213 & 25.977 & -0.201 & 0.58900 & -1.0310 \\
\hline-5593.151 & 34.875 & 44.148 & 25.774 & -0.179 & 0.80900 & -1.0250 \\
\hline-5589.143 & 34.375 & 44.144 & 25.590 & -0.149 & 1.0510 & -0.98400 \\
\hline-5585.135 & 34.125 & 44.323 & 25.460 & -0.110 & 1.2350 & -0.9420 \\
\hline
\end{tabular}




\begin{tabular}{|c|c|c|c|c|c|c|}
\hline-5581.127 & 33.875 & 45.170 & 25.392 & -0.0603 & 1.3900 & -0.88600 \\
\hline-5577.119 & 33.875 & 46.149 & 25.316 & 0.00100 & 1.4820 & -0.83100 \\
\hline-5573.111 & 33.875 & 46.861 & 25.331 & 0.0754 & 1.5630 & -0.77800 \\
\hline-5569.103 & 34.125 & 47.482 & 25.452 & 0.172 & 1.6080 & -0.71500 \\
\hline-5565.094 & 34.625 & 47.935 & 25.539 & 0.290 & 1.6680 & -0.64300 \\
\hline-5561.086 & 35.375 & 48.371 & 25.651 & 0.370 & 1.7000 & -0.56400 \\
\hline-5557.078 & 36.875 & 48.708 & 25.884 & 0.365 & 1.6990 & -0.48600 \\
\hline-5553.070 & 38.875 & 48.984 & 26.142 & 0.308 & 1.6840 & -0.41400 \\
\hline-5549.062 & 40.875 & 49.224 & 26.259 & 0.248 & 1.6350 & -0.35600 \\
\hline-5545.054 & 42.125 & 49.416 & 26.465 & 0.194 & 1.5430 & -0.31600 \\
\hline-5541.046 & 42.625 & 49.516 & 26.820 & 0.146 & 1.4370 & -0.28800 \\
\hline-5537.038 & 42.875 & 49.560 & 27.322 & 0.103 & 1.2870 & -0.27100 \\
\hline-5533.029 & 42.875 & 49.491 & 27.977 & 0.0658 & 1.1760 & -0.26300 \\
\hline-5529.021 & 42.875 & 49.391 & 28.841 & 0.0346 & 1.0380 & -0.26000 \\
\hline-5525.013 & 42.875 & 49.224 & 29.863 & 0.00890 & 0.91100 & -0.25900 \\
\hline-5521.005 & 42.875 & 49.060 & 30.979 & -0.0126 & 0.79000 & -0.25800 \\
\hline-5516.997 & 42.625 & 48.871 & 32.283 & -0.0310 & 0.68800 & -0.25700 \\
\hline-5512.989 & 42.625 & 48.669 & 33.734 & -0.0467 & 0.59800 & -0.25700 \\
\hline-5508.981 & 42.375 & 48.403 & 34.751 & -0.0597 & 0.51000 & -0.25600 \\
\hline-5504.973 & 42.125 & 48.124 & 35.245 & -0.0699 & 0.42700 & -0.25400 \\
\hline-5500.965 & 41.875 & 47.835 & 35.300 & -0.0774 & 0.35200 & -0.25200 \\
\hline-5496.956 & 41.625 & 47.494 & 35.225 & -0.0823 & 0.29300 & -0.24900 \\
\hline-5492.948 & 41.125 & 47.159 & 34.989 & -0.0856 & 0.24000 & -0.24600 \\
\hline-5488.940 & 40.875 & 46.821 & 34.723 & -0.0882 & 0.19900 & -0.24200 \\
\hline-5484.932 & 40.625 & 46.466 & 34.429 & -0.0904 & 0.15900 & -0.23800 \\
\hline-5480.924 & 40.125 & 46.130 & 34.154 & -0.0920 & 0.11500 & -0.23300 \\
\hline-5476.916 & 39.875 & 45.747 & 33.899 & -0.0932 & -0.0030000 & -0.22800 \\
\hline-5472.908 & 39.625 & 45.389 & 33.679 & -0.0939 & -0.0080000 & -0.22300 \\
\hline-5468.900 & 39.125 & 45.011 & 33.478 & -0.0941 & -0.0090000 & -0.21800 \\
\hline-5464.891 & 38.875 & 44.611 & 33.294 & -0.0939 & -0.0090000 & -0.21300 \\
\hline-5460.883 & 38.625 & 44.222 & 33.141 & -0.0931 & -0.0090000 & -0.20800 \\
\hline-5456.875 & 38.125 & 43.819 & 33.000 & -0.0919 & -0.0080000 & -0.20200 \\
\hline-5452.867 & 37.875 & 43.392 & 32.862 & -0.0902 & -0.0080000 & -0.19700 \\
\hline-5448.859 & 37.375 & 42.968 & 32.749 & -0.0880 & -0.0080000 & -0.19200 \\
\hline-5444.851 & 37.125 & 42.538 & 32.644 & -0.0853 & -0.0080000 & -0.18600 \\
\hline-5440.843 & 36.875 & 42.121 & 32.546 & -0.0821 & -0.0070000 & -0.18200 \\
\hline-5436.835 & 36.375 & 41.706 & 32.457 & -0.0785 & -0.0070000 & -0.17700 \\
\hline-5432.827 & 36.125 & 41.296 & 32.369 & -0.0744 & -0.0070000 & -0.17100 \\
\hline-5428.818 & 35.875 & 40.903 & 32.283 & -0.0702 & -0.0060000 & -0.16600 \\
\hline-5424.810 & 35.375 & 40.512 & 32.206 & -0.0661 & -0.0060000 & -0.16000 \\
\hline-5420.802 & 35.125 & 40.138 & 32.130 & -0.0623 & -0.0060000 & -0.15500 \\
\hline-5416.794 & 34.875 & 39.766 & 32.058 & -0.0586 & -0.0060000 & -0.14900 \\
\hline-5412.786 & 34.625 & 39.417 & 31.989 & -0.0551 & -0.0050000 & -0.14300 \\
\hline-5408.778 & 34.375 & 39.084 & 31.921 & -0.0519 & -0.0050000 & -0.13800 \\
\hline-5404.770 & 34.125 & 38.755 & 31.858 & -0.0488 & -0.0050000 & -0.13400 \\
\hline-5400.762 & 33.875 & 38.435 & 31.792 & -0.0459 & -0.0040000 & -0.12900 \\
\hline-5396.754 & 33.625 & 38.114 & 31.727 & -0.0432 & -0.0040000 & -0.12500 \\
\hline-5392.745 & 33.625 & 37.803 & 31.669 & -0.0408 & -0.0040000 & -0.12000 \\
\hline-5388.737 & 33.375 & 37.496 & 31.607 & -0.0385 & -0.0040000 & -0.11600 \\
\hline-5384.729 & 33.125 & 37.205 & 31.551 & -0.0364 & -0.0030000 & -0.11200 \\
\hline-5380.721 & 33.125 & 36.909 & 31.494 & -0.0346 & -0.0030000 & -0.10800 \\
\hline
\end{tabular}




\begin{tabular}{|c|c|c|c|c|c|c|}
\hline-5376.713 & 32.875 & 36.626 & 31.437 & -0.0329 & -0.0030000 & -0.10400 \\
\hline-5372.705 & 32.875 & 36.349 & 31.382 & -0.0314 & -0.0030000 & -0.10000 \\
\hline-5368.697 & 32.875 & 36.078 & 31.327 & -0.0300 & -0.0020000 & -0.097000 \\
\hline-5364.689 & 32.625 & 35.811 & 31.271 & -0.0287 & -0.0020000 & -0.094000 \\
\hline-5360.680 & 32.625 & 35.550 & 31.220 & -0.0275 & -0.0020000 & -0.091000 \\
\hline-5356.672 & 32.625 & 35.295 & 31.162 & -0.0262 & -0.0020000 & -0.088000 \\
\hline-5352.664 & 32.375 & 35.048 & 31.104 & -0.0250 & -0.0010000 & -0.085000 \\
\hline-5348.656 & 32.375 & 34.802 & 31.049 & -0.0239 & -0.0010000 & -0.082000 \\
\hline-5344.648 & 32.375 & 34.564 & 30.986 & -0.0228 & -0.0010000 & -0.080000 \\
\hline-5340.640 & 32.125 & 34.326 & 30.921 & -0.0217 & 0.0000 & -0.078000 \\
\hline-5336.632 & 32.125 & 34.091 & 30.851 & -0.0207 & 0.0000 & -0.076000 \\
\hline-5332.624 & 32.125 & 33.860 & 30.781 & -0.0197 & 0.0000 & -0.074000 \\
\hline-5328.616 & 32.125 & 33.637 & 30.703 & -0.0188 & 0.0010000 & -0.073000 \\
\hline-5324.607 & 31.875 & 33.422 & 30.631 & -0.0179 & 0.0010000 & -0.072000 \\
\hline-5320.599 & 31.875 & 33.217 & 30.542 & -0.0171 & 0.0020000 & -0.070000 \\
\hline-5316.591 & 31.875 & 33.030 & 30.445 & -0.0162 & 0.0020000 & -0.069000 \\
\hline-5312.583 & 31.875 & 32.868 & 30.337 & -0.0155 & 0.0030000 & -0.068000 \\
\hline-5308.575 & 31.625 & 32.727 & 30.222 & -0.0148 & 0.0030000 & -0.067000 \\
\hline-5304.567 & 31.625 & 32.609 & 30.091 & -0.0141 & 0.0040000 & -0.067000 \\
\hline-5300.559 & 31.625 & 32.511 & 29.955 & -0.0134 & 0.0050000 & -0.066000 \\
\hline-5296.551 & 31.625 & 32.430 & 29.805 & -0.0128 & 0.0050000 & -0.065000 \\
\hline-5292.542 & 31.375 & 32.363 & 29.654 & -0.0123 & 0.0060000 & -0.065000 \\
\hline-5288.534 & 31.375 & 32.307 & 29.502 & -0.0118 & 0.0070000 & -0.064000 \\
\hline-5284.526 & 31.375 & 32.257 & 29.344 & -0.0113 & 0.0080000 & -0.064000 \\
\hline-5280.518 & 31.375 & 32.210 & 29.185 & -0.0108 & 0.0090000 & -0.063000 \\
\hline-5276.510 & 31.125 & 32.171 & 29.038 & -0.0105 & 0.0100000 & -0.062000 \\
\hline-5272.502 & 31.125 & 32.136 & 28.896 & -0.0101 & 0.011000 & -0.062000 \\
\hline-5268.494 & 31.125 & 32.102 & 28.751 & -0.00980 & 0.013000 & -0.061000 \\
\hline-5264.486 & 31.125 & 32.070 & 28.616 & -0.00950 & 0.014000 & -0.060000 \\
\hline-5260.478 & 31.125 & 32.041 & 28.494 & -0.00930 & 0.016000 & -0.059000 \\
\hline-5256.469 & 31.125 & 32.014 & 28.385 & -0.00910 & 0.018000 & -0.058000 \\
\hline-5252.461 & 30.875 & 31.991 & 28.281 & -0.00900 & 0.020000 & -0.057000 \\
\hline-5248.453 & 30.875 & 31.968 & 28.165 & -0.00890 & 0.021000 & -0.056000 \\
\hline-5244.445 & 30.875 & 31.948 & 28.063 & -0.00880 & 0.023000 & -0.054000 \\
\hline-5240.437 & 30.875 & 31.933 & 27.981 & -0.00870 & 0.025000 & -0.053000 \\
\hline-5236.429 & 30.875 & 31.919 & 27.895 & -0.00860 & 0.026000 & -0.052000 \\
\hline-5232.421 & 30.875 & 31.910 & 27.819 & -0.00850 & 0.028000 & -0.050000 \\
\hline-5228.413 & 30.625 & 31.897 & 27.746 & -0.00850 & 0.030000 & -0.049000 \\
\hline-5224.405 & 30.625 & 31.894 & 27.677 & -0.00840 & 0.031000 & -0.047000 \\
\hline-5220.396 & 30.625 & 31.894 & 27.626 & -0.00830 & 0.033000 & -0.046000 \\
\hline-5216.388 & 30.625 & 31.894 & 27.580 & -0.00820 & 0.034000 & -0.043000 \\
\hline-5212.380 & 30.625 & 31.904 & 27.547 & -0.00810 & 0.036000 & -0.042000 \\
\hline-5208.372 & 30.625 & 31.918 & 27.513 & -0.00800 & 0.037000 & -0.040000 \\
\hline-5204.364 & 30.625 & 31.937 & 27.502 & -0.00790 & 0.039000 & -0.038000 \\
\hline-5200.356 & 30.375 & 31.967 & 27.485 & -0.00780 & 0.041000 & -0.036000 \\
\hline-5196.348 & 30.375 & 31.986 & 27.496 & -0.00770 & 0.042000 & -0.035000 \\
\hline-5192.340 & 30.375 & 32.017 & 27.492 & -0.00760 & 0.044000 & -0.034000 \\
\hline-5188.331 & 30.375 & 32.067 & 27.500 & -0.00740 & 0.045000 & -0.033000 \\
\hline-5184.323 & 30.375 & 32.113 & 27.510 & -0.00730 & 0.047000 & -0.032000 \\
\hline-5180.315 & 30.375 & 32.153 & 27.523 & -0.00720 & 0.048000 & -0.031000 \\
\hline-5176.307 & 30.375 & 32.216 & 27.536 & -0.00710 & 0.050000 & -0.031000 \\
\hline
\end{tabular}




\begin{tabular}{|c|c|c|c|c|c|c|}
\hline-5172.299 & 30.375 & 32.278 & 27.549 & -0.00700 & 0.051000 & -0.030000 \\
\hline-5168.291 & 30.125 & 32.339 & 27.555 & -0.00690 & 0.053000 & -0.030000 \\
\hline-5164.283 & 30.125 & 32.414 & 27.572 & -0.00670 & 0.054000 & -0.030000 \\
\hline-5160.275 & 30.125 & 32.476 & 27.582 & -0.00660 & 0.056000 & -0.029000 \\
\hline-5156.267 & 30.125 & 32.557 & 27.590 & -0.00650 & 0.058000 & -0.029000 \\
\hline-5152.258 & 30.125 & 32.648 & 27.596 & -0.00630 & 0.059000 & -0.029000 \\
\hline-5148.250 & 30.125 & 32.726 & 27.605 & -0.00620 & 0.061000 & -0.029000 \\
\hline-5144.242 & 30.125 & 32.812 & 27.618 & -0.00610 & 0.063000 & -0.029000 \\
\hline-5140.234 & 30.125 & 32.919 & 27.621 & -0.00590 & 0.066000 & -0.029000 \\
\hline-5136.226 & 30.125 & 33.028 & 27.623 & -0.00580 & 0.068000 & -0.030000 \\
\hline-5132.218 & 30.125 & 33.148 & 27.621 & -0.00570 & 0.071000 & -0.030000 \\
\hline-5128.210 & 30.125 & 33.263 & 27.609 & -0.00550 & 0.074000 & -0.030000 \\
\hline-5124.202 & 30.125 & 33.399 & 27.602 & -0.00540 & 0.077000 & -0.031000 \\
\hline-5120.193 & 29.875 & 33.547 & 27.583 & -0.00520 & 0.081000 & -0.032000 \\
\hline-5116.185 & 29.875 & 33.703 & 27.560 & -0.00510 & 0.085000 & -0.033000 \\
\hline-5112.177 & 29.875 & 33.866 & 27.539 & -0.00490 & 0.089000 & -0.034000 \\
\hline-5108.169 & 29.875 & 34.052 & 27.507 & -0.00480 & 0.093000 & -0.036000 \\
\hline-5104.161 & 29.875 & 34.234 & 27.484 & -0.00460 & 0.10000 & -0.037000 \\
\hline-5100.153 & 29.875 & 34.449 & 27.441 & -0.00440 & 0.10500 & -0.039000 \\
\hline-5096.145 & 29.875 & 34.718 & 27.398 & -0.00430 & 0.11200 & -0.041000 \\
\hline-5092.137 & 29.875 & 35.010 & 27.360 & -0.00410 & 0.12300 & -0.043000 \\
\hline-5088.129 & 29.875 & 35.321 & 27.318 & -0.00390 & 0.13300 & -0.045000 \\
\hline-5084.120 & 29.875 & 35.682 & 27.272 & -0.00380 & 0.14400 & -0.047000 \\
\hline-5080.112 & 29.875 & 36.075 & 27.223 & -0.00360 & 0.15700 & -0.050000 \\
\hline-5076.104 & 29.875 & 36.549 & 27.167 & -0.00340 & 0.17300 & -0.052000 \\
\hline-5072.096 & 29.875 & 37.101 & 27.111 & -0.00330 & 0.18900 & -0.055000 \\
\hline-5068.088 & 29.875 & 37.700 & 27.054 & -0.00310 & 0.20900 & -0.058000 \\
\hline-5064.080 & 29.875 & 38.329 & 26.994 & -0.00290 & 0.22900 & -0.062000 \\
\hline-5060.072 & 29.875 & 39.047 & 26.919 & -0.00270 & 0.24900 & -0.066000 \\
\hline-5056.064 & 29.875 & 39.795 & 26.849 & -0.00250 & 0.27400 & -0.070000 \\
\hline-5052.056 & 29.875 & 40.701 & 26.783 & -0.00230 & 0.30100 & -0.074000 \\
\hline-5048.047 & 29.875 & 41.637 & 26.702 & -0.00220 & 0.33100 & -0.080000 \\
\hline-5044.039 & 29.875 & 42.664 & 26.616 & -0.00200 & 0.36300 & -0.086000 \\
\hline-5040.031 & 29.875 & 43.730 & 26.524 & -0.00180 & 0.40200 & -0.094000 \\
\hline-5036.023 & 29.625 & 44.850 & 26.419 & -0.00160 & 0.44300 & -0.10200 \\
\hline-5032.015 & 29.625 & 46.078 & 26.300 & -0.00140 & 0.49000 & -0.11100 \\
\hline-5028.007 & 29.625 & 47.375 & 26.179 & -0.00120 & 0.54600 & -0.12100 \\
\hline-5023.999 & 29.625 & 48.775 & 26.042 & -0.00100 & 0.61300 & -0.13300 \\
\hline-5019.991 & 29.625 & 50.316 & 25.887 & -0.000800 & 0.69800 & -0.14800 \\
\hline-5015.982 & 29.625 & 51.914 & 25.693 & -0.000600 & 0.80000 & -0.16800 \\
\hline-5011.974 & 29.625 & 53.750 & 25.496 & -0.000400 & 0.96300 & -0.19600 \\
\hline-5007.966 & 29.625 & 55.816 & 25.210 & $-1.00 e-04$ & 1.1780 & -0.23100 \\
\hline-5003.958 & 29.625 & 58.572 & 24.775 & $1.00 \mathrm{e}-04$ & 1.5880 & -0.28300 \\
\hline-4999.950 & 29.625 & 63.706 & 23.872 & 0.000300 & 2.4740 & -0.36800 \\
\hline
\end{tabular}




\begin{tabular}{|c|c|c|c|c|c|c|}
\hline-5401.825 & 31.375 & 34.168 & 27.977 & 0.0636 & 0.19700 & -0.098000 \\
\hline-5397.810 & 31.625 & 34.538 & 28.203 & 0.0700 & 0.20400 & -0.082000 \\
\hline-5393.796 & 31.875 & 34.944 & 28.412 & 0.0748 & 0.21100 & -0.071000 \\
\hline-5389.781 & 32.125 & 35.409 & 28.609 & 0.0782 & 0.21600 & -0.061000 \\
\hline-5385.766 & 32.375 & 35.860 & 28.799 & 0.0801 & 0.22300 & -0.053000 \\
\hline-5381.752 & 32.625 & 36.327 & 28.986 & 0.0805 & 0.22700 & -0.044000 \\
\hline-5377.737 & 33.125 & 36.790 & 29.225 & 0.0795 & 0.23000 & -0.035000 \\
\hline-5373.723 & 33.375 & 37.245 & 29.495 & 0.0778 & 0.23100 & -0.026000 \\
\hline-5369.708 & 33.625 & 37.745 & 29.775 & 0.0761 & 0.22900 & -0.021000 \\
\hline-5365.693 & 33.875 & 38.189 & 30.145 & 0.0744 & 0.22700 & -0.014000 \\
\hline-5361.679 & 34.125 & 38.621 & 30.574 & 0.0729 & 0.22300 & -0.0100000 \\
\hline-5357.664 & 34.375 & 39.014 & 31.076 & 0.0714 & 0.21800 & -0.0060000 \\
\hline-5353.650 & 34.625 & 39.319 & 31.604 & 0.0700 & 0.20700 & -0.0050000 \\
\hline-5349.635 & 34.875 & 39.559 & 32.111 & 0.0687 & 0.19600 & -0.0040000 \\
\hline-5345.620 & 35.125 & 39.760 & 32.580 & 0.0676 & 0.18500 & -0.0030000 \\
\hline-5341.606 & 35.375 & 39.907 & 33.013 & 0.0665 & 0.17300 & -0.0030000 \\
\hline-5337.591 & 35.625 & 40.042 & 33.415 & 0.0654 & 0.16200 & -0.0040000 \\
\hline-5333.577 & 35.875 & 40.127 & 33.779 & 0.0645 & 0.15100 & -0.0060000 \\
\hline-5329.562 & 36.375 & 40.168 & 34.131 & 0.0637 & 0.14100 & -0.0070000 \\
\hline-5325.547 & 36.625 & 40.193 & 34.471 & 0.0630 & 0.13300 & -0.0070000 \\
\hline-5321.533 & 36.875 & 40.226 & 34.795 & 0.0623 & 0.12600 & -0.0070000 \\
\hline-5317.518 & 37.125 & 40.297 & 35.098 & 0.0617 & 0.12000 & -0.0070000 \\
\hline-5313.504 & 37.375 & 40.387 & 35.389 & 0.0613 & 0.11500 & -0.0080000 \\
\hline-5309.489 & 37.625 & 40.499 & 35.668 & 0.0609 & 0.11100 & -0.0070000 \\
\hline-5305.474 & 37.875 & 40.599 & 35.940 & 0.0606 & 0.10800 & -0.0060000 \\
\hline-5301.460 & 38.125 & 40.730 & 36.208 & 0.0602 & 0.10500 & -0.0030000 \\
\hline-5297.445 & 38.375 & 40.883 & 36.468 & 0.0599 & 0.10300 & -0.0020000 \\
\hline-5293.431 & 38.625 & 41.047 & 36.725 & 0.0597 & 0.10100 & 0.0000 \\
\hline-5289.416 & 38.875 & 41.232 & 36.972 & 0.0594 & 0.099000 & 0.0030000 \\
\hline-5285.401 & 39.125 & 41.429 & 37.214 & 0.0591 & 0.098000 & 0.0050000 \\
\hline-5281.387 & 39.125 & 41.633 & 37.453 & 0.0589 & 0.097000 & 0.0070000 \\
\hline-5277.372 & 39.375 & 41.841 & 37.678 & 0.0586 & 0.096000 & 0.0100000 \\
\hline-5273.358 & 39.625 & 42.066 & 37.901 & 0.0584 & 0.095000 & 0.011000 \\
\hline-5269.343 & 39.875 & 42.293 & 38.123 & 0.0582 & 0.094000 & 0.014000 \\
\hline-5265.328 & 40.125 & 42.522 & 38.337 & 0.0580 & 0.094000 & 0.015000 \\
\hline-5261.314 & 40.375 & 42.762 & 38.547 & 0.0578 & 0.093000 & 0.016000 \\
\hline-5257.299 & 40.625 & 42.999 & 38.750 & 0.0576 & 0.093000 & 0.018000 \\
\hline-5253.285 & 40.875 & 43.245 & 38.951 & 0.0575 & 0.093000 & 0.019000 \\
\hline-5249.270 & 41.125 & 43.501 & 39.144 & 0.0573 & 0.093000 & 0.019000 \\
\hline-5245.255 & 41.375 & 43.749 & 39.342 & 0.0572 & 0.092000 & 0.019000 \\
\hline-5241.241 & 41.625 & 43.993 & 39.551 & 0.0571 & 0.092000 & 0.018000 \\
\hline-5237.226 & 41.875 & 44.233 & 39.747 & 0.0570 & 0.092000 & 0.018000 \\
\hline-5233.212 & 42.125 & 44.462 & 39.962 & 0.0569 & 0.092000 & 0.018000 \\
\hline-5229.197 & 42.375 & 44.708 & 40.186 & 0.0568 & 0.092000 & 0.017000 \\
\hline-5225.182 & 42.625 & 44.939 & 40.409 & 0.0567 & 0.092000 & 0.016000 \\
\hline-5221.168 & 42.875 & 45.167 & 40.634 & 0.0566 & 0.092000 & 0.015000 \\
\hline-5217.153 & 43.125 & 45.370 & 40.859 & 0.0566 & 0.092000 & 0.013000 \\
\hline-5213.139 & 43.375 & 45.573 & 41.084 & 0.0566 & 0.092000 & 0.011000 \\
\hline-5209.124 & 43.625 & 45.771 & 41.304 & 0.0565 & 0.091000 & 0.0090000 \\
\hline-5205.109 & 43.875 & 45.962 & 41.535 & 0.0565 & 0.091000 & 0.0060000 \\
\hline-5201.095 & 43.875 & 46.142 & 41.755 & 0.0565 & 0.091000 & 0.0020000 \\
\hline
\end{tabular}




\begin{tabular}{|c|c|c|c|c|c|c|}
\hline-5197.080 & 44.125 & 46.333 & 41.974 & 0.0566 & 0.090000 & -0.0010000 \\
\hline-5193.066 & 44.375 & 46.540 & 42.182 & 0.0566 & 0.090000 & -0.0050000 \\
\hline-5189.051 & 44.625 & 46.766 & 42.385 & 0.0566 & 0.091000 & -0.0080000 \\
\hline-5185.036 & 44.875 & 47.007 & 42.581 & 0.0567 & 0.092000 & -0.012000 \\
\hline-5181.022 & 45.125 & 47.267 & 42.774 & 0.0568 & 0.093000 & -0.016000 \\
\hline-5177.007 & 45.375 & 47.526 & 42.954 & 0.0568 & 0.094000 & -0.021000 \\
\hline-5172.993 & 45.625 & 47.796 & 43.132 & 0.0569 & 0.096000 & -0.024000 \\
\hline-5168.978 & 45.625 & 48.087 & 43.297 & 0.0570 & 0.099000 & -0.028000 \\
\hline-5164.964 & 45.875 & 48.379 & 43.458 & 0.0571 & 0.10200 & -0.032000 \\
\hline-5160.949 & 46.125 & 48.675 & 43.601 & 0.0571 & 0.10700 & -0.036000 \\
\hline-5156.934 & 46.375 & 48.977 & 43.727 & 0.0571 & 0.11200 & -0.039000 \\
\hline-5152.920 & 46.625 & 49.295 & 43.847 & 0.0571 & 0.11900 & -0.042000 \\
\hline-5148.905 & 46.625 & 49.627 & 43.952 & 0.0570 & 0.12700 & -0.043000 \\
\hline-5144.891 & 46.875 & 49.982 & 44.043 & 0.0569 & 0.13400 & -0.046000 \\
\hline-5140.876 & 47.125 & 50.346 & 44.141 & 0.0568 & 0.14300 & -0.048000 \\
\hline-5136.861 & 47.375 & 50.722 & 44.232 & 0.0566 & 0.15300 & -0.052000 \\
\hline-5132.847 & 47.625 & 51.111 & 44.280 & 0.0564 & 0.16300 & -0.056000 \\
\hline-5128.832 & 47.875 & 51.520 & 44.346 & 0.0562 & 0.17400 & -0.059000 \\
\hline-5124.818 & 48.125 & 51.958 & 44.414 & 0.0560 & 0.18200 & -0.066000 \\
\hline-5120.803 & 48.375 & 52.430 & 44.449 & 0.0557 & 0.19400 & -0.072000 \\
\hline-5116.788 & 48.625 & 52.901 & 44.466 & 0.0554 & 0.20300 & -0.080000 \\
\hline-5112.774 & 48.875 & 53.373 & 44.469 & 0.0551 & 0.21600 & -0.091000 \\
\hline-5108.759 & 49.125 & 53.902 & 44.440 & 0.0547 & 0.22400 & -0.10000 \\
\hline-5104.745 & 49.125 & 54.388 & 44.374 & 0.0543 & 0.22800 & -0.11300 \\
\hline-5100.730 & 49.375 & 54.803 & 44.302 & 0.0539 & 0.23600 & -0.12500 \\
\hline-5096.715 & 49.625 & 55.193 & 44.216 & 0.0534 & 0.24100 & -0.13500 \\
\hline-5092.701 & 49.875 & 55.583 & 44.193 & 0.0530 & 0.24600 & -0.15000 \\
\hline-5088.686 & 50.125 & 55.967 & 44.159 & 0.0524 & 0.25100 & -0.16400 \\
\hline-5084.672 & 50.375 & 56.281 & 44.114 & 0.0519 & 0.25500 & -0.18000 \\
\hline-5080.657 & 50.625 & 56.622 & 44.039 & 0.0513 & 0.25900 & -0.19100 \\
\hline-5076.642 & 50.875 & 56.938 & 44.027 & 0.0507 & 0.26500 & -0.20700 \\
\hline-5072.628 & 51.125 & 57.226 & 43.935 & 0.0501 & 0.26900 & -0.22400 \\
\hline-5068.613 & 51.125 & 57.488 & 43.888 & 0.0494 & 0.26900 & -0.24200 \\
\hline-5064.599 & 51.375 & 57.781 & 43.756 & 0.0487 & 0.27000 & -0.25700 \\
\hline-5060.584 & 51.625 & 58.058 & 43.564 & 0.0480 & 0.27600 & -0.27600 \\
\hline-5056.569 & 51.875 & 58.329 & 43.352 & 0.0473 & 0.27900 & -0.29600 \\
\hline-5052.555 & 52.125 & 58.610 & 43.103 & 0.0465 & 0.28600 & -0.31500 \\
\hline-5048.540 & 52.125 & 58.878 & 42.755 & 0.0457 & 0.28600 & -0.33300 \\
\hline-5044.526 & 52.375 & 59.133 & 42.302 & 0.0448 & 0.29000 & -0.35300 \\
\hline-5040.511 & 52.625 & 59.385 & 41.915 & 0.0440 & 0.29300 & -0.37100 \\
\hline-5036.496 & 52.625 & 59.629 & 41.351 & 0.0430 & 0.29600 & -0.39100 \\
\hline-5032.482 & 52.875 & 59.897 & 40.662 & 0.0420 & 0.30400 & -0.41300 \\
\hline-5028.467 & 53.125 & 60.110 & 40.023 & 0.0409 & 0.31300 & -0.43400 \\
\hline-5024.453 & 53.125 & 60.361 & 39.382 & 0.0396 & 0.32400 & -0.45200 \\
\hline-5020.438 & 53.375 & 60.629 & 38.706 & 0.0382 & 0.33400 & -0.47200 \\
\hline-5016.423 & 53.625 & 60.841 & 38.064 & 0.0366 & 0.34300 & -0.48900 \\
\hline-5012.409 & 53.625 & 61.108 & 37.440 & 0.0348 & 0.35300 & -0.50100 \\
\hline-5008.394 & 53.875 & 61.331 & 36.771 & 0.0329 & 0.36200 & -0.52000 \\
\hline-5004.380 & 53.875 & 61.576 & 36.117 & 0.0309 & 0.37100 & -0.53700 \\
\hline-5000.365 & 54.125 & 61.762 & 35.443 & 0.0287 & 0.37500 & -0.55600 \\
\hline-4996.350 & 54.125 & 61.924 & 34.759 & 0.0263 & 0.38000 & -0.57400 \\
\hline
\end{tabular}




$\begin{array}{lllllll}-4992.336 & 54.375 & 62.099 & 34.219 & 0.0238 & 0.39100 & -0.58000 \\ -4988.321 & 54.375 & 62.289 & 33.678 & 0.0212 & 0.40200 & -0.59900 \\ -4984.307 & 54.375 & 62.445 & 33.181 & 0.0183 & 0.41300 & -0.61300 \\ -4980.292 & 54.375 & 62.629 & 32.741 & 0.0154 & 0.42100 & -0.62500 \\ -4976.277 & 54.625 & 62.792 & 32.348 & 0.0123 & 0.43000 & -0.63700 \\ -4972.263 & 54.625 & 62.971 & 31.862 & 0.00900 & 0.43600 & -0.65400 \\ -4968.248 & 54.625 & 63.154 & 31.404 & 0.00560 & 0.44500 & -0.67000 \\ -4964.234 & 54.625 & 63.387 & 30.987 & 0.00210 & 0.45900 & -0.67800 \\ -4960.219 & 54.625 & 63.573 & 30.627 & -0.00170 & 0.47700 & -0.69100 \\ -4956.204 & 54.625 & 63.781 & 30.285 & -0.00550 & 0.48100 & -0.71200 \\ -4952.190 & 54.625 & 63.979 & 29.943 & -0.00950 & 0.49300 & -0.73000 \\ -4948.175 & 54.375 & 64.181 & 29.574 & -0.0137 & 0.51700 & -0.74900 \\ -4944.161 & 54.375 & 64.370 & 29.207 & -0.0180 & 0.54500 & -0.76600 \\ -4940.146 & 54.375 & 64.578 & 28.918 & -0.0225 & 0.57400 & -0.78800 \\ -4936.131 & 54.125 & 64.770 & 28.647 & -0.0271 & 0.60500 & -0.82400 \\ -4932.117 & 54.125 & 64.986 & 28.354 & -0.0319 & 0.64600 & -0.86500 \\ -4928.102 & 53.875 & 65.223 & 28.036 & -0.0368 & 0.68900 & -0.92200 \\ -4924.088 & 53.875 & 65.458 & 27.781 & -0.0418 & 0.75100 & -0.99400 \\ -4920.073 & 53.625 & 65.710 & 27.395 & -0.0471 & 0.82800 & -1.0860 \\ -4916.058 & 53.375 & 65.991 & 26.940 & -0.0524 & 0.92500 & -1.2140 \\ -4912.044 & 53.125 & 66.275 & 26.368 & -0.0580 & 1.0800 & -1.4200 \\ -4908.029 & 52.875 & 66.654 & 25.563 & -0.0636 & 1.3240 & -1.7500 \\ -4904.015 & 52.875 & 67.189 & 24.387 & -0.0695 & 1.7500 & -2.3820 \\ -4900.000 & 52.875 & 68.454 & 21.835 & -0.0754 & 2.9090 & -4.4410\end{array}$




\begin{tabular}{|c|c|c|c|c|c|c|}
\hline Date & & & & & & \\
\hline Date & ledian (muT) & ed_Int Upper & Cred_Int Lower & Fdt (muT/yr) & dFdt Upper & dFdt Lower \\
\hline-7000.000 & 51.375 & 68.625 & 21.707 & 0.0459 & 3.1340 & -2.2780 \\
\hline-6995.988 & 51.375 & 67.611 & 23.500 & 0.0445 & 1.9460 & -1.5350 \\
\hline-6991.977 & 51.625 & 67.175 & 24.348 & 0.0431 & 1.4770 & -1.1880 \\
\hline-6987.965 & 51.625 & 66.867 & 24.965 & 0.0418 & 1.2270 & -0.99500 \\
\hline-6983.953 & 51.875 & 66.646 & 25.436 & 0.0404 & 1.0630 & -0.86300 \\
\hline-6979.942 & 52.125 & 66.466 & 25.778 & 0.0390 & 0.94100 & -0.77600 \\
\hline-6975.930 & 52.125 & 66.337 & 26.065 & 0.0377 & 0.84600 & -0.70900 \\
\hline-6971.919 & 52.375 & 66.219 & 26.300 & 0.0364 & 0.78800 & -0.66000 \\
\hline-6967.907 & 52.625 & 66.094 & 26.516 & 0.0351 & 0.73700 & -0.61900 \\
\hline-6963.895 & 52.625 & 66.014 & 26.680 & 0.0338 & 0.69300 & -0.58400 \\
\hline-6959.884 & 52.875 & 65.927 & 26.842 & 0.0325 & 0.65800 & -0.55500 \\
\hline-6955.872 & 52.875 & 65.843 & 26.888 & 0.0313 & 0.62500 & -0.53300 \\
\hline-6951.860 & 53.125 & 65.761 & 26.993 & 0.0301 & 0.60100 & -0.51100 \\
\hline-6947.849 & 53.125 & 65.673 & 27.059 & 0.0288 & 0.58200 & -0.49800 \\
\hline-6943.837 & 53.375 & 65.622 & 27.105 & 0.0276 & 0.56100 & -0.48100 \\
\hline-6939.826 & 53.375 & 65.531 & 27.196 & 0.0264 & 0.54800 & -0.46900 \\
\hline-6935.814 & 53.625 & 65.479 & 27.211 & 0.0253 & 0.53900 & -0.45800 \\
\hline-6931.802 & 53.625 & 65.428 & 27.264 & 0.0241 & 0.52800 & -0.44700 \\
\hline-6927.791 & 53.625 & 65.353 & 27.389 & 0.0230 & 0.52200 & -0.43800 \\
\hline-6923.779 & 53.875 & 65.296 & 27.493 & 0.0218 & 0.51700 & -0.43600 \\
\hline-6919.767 & 53.875 & 65.220 & 27.515 & 0.0207 & 0.51500 & -0.43100 \\
\hline-6915.756 & 53.875 & 65.173 & 27.586 & 0.0196 & 0.51300 & -0.42400 \\
\hline-6911.744 & 54.125 & 65.116 & 27.628 & 0.0186 & 0.51100 & -0.41800 \\
\hline-6907.733 & 54.125 & 65.076 & 27.724 & 0.0175 & 0.51100 & -0.41400 \\
\hline-6903.721 & 54.125 & 65.020 & 27.779 & 0.0165 & 0.51200 & -0.40800 \\
\hline-6899.709 & 54.375 & 64.968 & 27.925 & 0.0154 & 0.51100 & -0.40600 \\
\hline-6895.698 & 54.375 & 64.932 & 27.984 & 0.0144 & 0.51000 & -0.40200 \\
\hline-6891.686 & 54.375 & 64.899 & 28.076 & 0.0134 & 0.50500 & -0.40100 \\
\hline-6887.674 & 54.375 & 64.854 & 28.133 & 0.0124 & 0.50600 & -0.40200 \\
\hline-6883.663 & 54.375 & 64.830 & 28.258 & 0.0115 & 0.49900 & -0.39800 \\
\hline-6879.651 & 54.375 & 64.801 & 28.361 & 0.0105 & 0.49200 & -0.39500 \\
\hline-6875.640 & 54.625 & 64.765 & 28.495 & 0.00960 & 0.49400 & -0.39500 \\
\hline-6871.628 & 54.625 & 64.709 & 28.565 & 0.00870 & 0.49400 & -0.39700 \\
\hline-6867.616 & 54.625 & 64.693 & 28.603 & 0.00780 & 0.48500 & -0.39300 \\
\hline-6863.605 & 54.625 & 64.641 & 28.674 & 0.00690 & 0.48100 & -0.38800 \\
\hline-6859.593 & 54.625 & 64.615 & 28.791 & 0.00600 & 0.48500 & -0.38600 \\
\hline-6855.581 & 54.625 & 64.584 & 28.863 & 0.00510 & 0.48800 & -0.38400 \\
\hline-6851.570 & 54.625 & 64.550 & 28.983 & 0.00430 & 0.48600 & -0.38000 \\
\hline-6847.558 & 54.625 & 64.497 & 29.093 & 0.00350 & 0.48900 & -0.37600 \\
\hline-6843.547 & 54.625 & 64.437 & 29.199 & 0.00270 & 0.48000 & -0.37600 \\
\hline-6839.535 & 54.625 & 64.374 & 29.310 & 0.00190 & 0.47700 & -0.37500 \\
\hline-6835.523 & 54.625 & 64.317 & 29.462 & 0.00110 & 0.47800 & -0.36900 \\
\hline-6831.512 & 54.625 & 64.296 & 29.617 & 0.000300 & 0.47500 & -0.36600 \\
\hline-6827.500 & 54.625 & 64.241 & 29.754 & -0.000400 & 0.47400 & -0.36100 \\
\hline-6823.488 & 54.625 & 64.181 & 29.929 & -0.00110 & 0.47300 & -0.35900 \\
\hline-6819.477 & 54.625 & 64.111 & 30.157 & -0.00180 & 0.46900 & -0.35600 \\
\hline-6815.465 & 54.625 & 64.025 & 30.341 & -0.00250 & 0.46800 & -0.35100 \\
\hline-6811.453 & 54.625 & 63.966 & 30.498 & -0.00320 & 0.46600 & -0.34800 \\
\hline
\end{tabular}




\begin{tabular}{|c|c|c|c|c|c|c|}
\hline-6807.442 & 54.625 & 63.900 & 30.678 & -0.00390 & 0.46000 & -0.34600 \\
\hline-6803.430 & 54.625 & 63.821 & 30.891 & -0.00450 & 0.46400 & -0.33600 \\
\hline-6799.419 & 54.625 & 63.756 & 31.065 & -0.00520 & 0.46400 & -0.32700 \\
\hline-6795.407 & 54.625 & 63.664 & 31.362 & -0.00580 & 0.46000 & -0.32300 \\
\hline-6791.395 & 54.625 & 63.571 & 31.569 & -0.00640 & 0.45900 & -0.31400 \\
\hline-6787.384 & 54.625 & 63.494 & 31.846 & -0.00700 & 0.45700 & -0.30700 \\
\hline-6783.372 & 54.625 & 63.422 & 32.177 & -0.00750 & 0.45400 & -0.30200 \\
\hline-6779.360 & 54.625 & 63.325 & 32.499 & -0.00810 & 0.45100 & -0.29400 \\
\hline-6775.349 & 54.625 & 63.242 & 32.874 & -0.00860 & 0.44600 & -0.29000 \\
\hline-6771.337 & 54.625 & 63.112 & 33.177 & -0.00920 & 0.44400 & -0.28500 \\
\hline-6767.326 & 54.625 & 63.006 & 33.589 & -0.00970 & 0.44100 & -0.27500 \\
\hline-6763.314 & 54.375 & 62.863 & 33.998 & -0.0101 & 0.43300 & -0.27000 \\
\hline-6759.302 & 54.375 & 62.743 & 34.398 & -0.0106 & 0.42900 & -0.26200 \\
\hline-6755.291 & 54.375 & 62.629 & 34.925 & -0.0111 & 0.42000 & -0.25500 \\
\hline-6751.279 & 54.375 & 62.473 & 35.442 & -0.0115 & 0.41100 & -0.25000 \\
\hline-6747.267 & 54.375 & 62.317 & 35.994 & -0.0119 & 0.40500 & -0.24500 \\
\hline-6743.256 & 54.375 & 62.143 & 36.612 & -0.0124 & 0.40000 & -0.24100 \\
\hline-6739.244 & 54.125 & 61.972 & 37.262 & -0.0128 & 0.38900 & -0.23700 \\
\hline-6735.233 & 54.125 & 61.773 & 37.939 & -0.0131 & 0.37900 & -0.23400 \\
\hline-6731.221 & 54.125 & 61.528 & 38.632 & -0.0135 & 0.36900 & -0.23200 \\
\hline-6727.209 & 54.125 & 61.297 & 39.342 & -0.0139 & 0.36200 & -0.22800 \\
\hline-6723.198 & 53.875 & 61.035 & 40.150 & -0.0143 & 0.35200 & -0.22900 \\
\hline-6719.186 & 53.875 & 60.778 & 40.817 & -0.0146 & 0.33600 & -0.22900 \\
\hline-6715.174 & 53.875 & 60.490 & 41.633 & -0.0150 & 0.32500 & -0.22800 \\
\hline-6711.163 & 53.875 & 60.255 & 42.504 & -0.0153 & 0.31200 & -0.22600 \\
\hline-6707.151 & 53.625 & 60.027 & 43.313 & -0.0157 & 0.30100 & -0.22500 \\
\hline-6703.140 & 53.625 & 59.810 & 43.908 & -0.0160 & 0.28800 & -0.22700 \\
\hline-6699.128 & 53.625 & 59.568 & 44.483 & -0.0164 & 0.27500 & -0.22900 \\
\hline-6695.116 & 53.625 & 59.367 & 44.954 & -0.0167 & 0.26400 & -0.23000 \\
\hline-6691.105 & 53.375 & 59.183 & 45.311 & -0.0170 & 0.25300 & -0.23100 \\
\hline-6687.093 & 53.375 & 59.044 & 45.523 & -0.0173 & 0.24200 & -0.23300 \\
\hline-6683.081 & 53.375 & 58.865 & 45.643 & -0.0177 & 0.23100 & -0.23600 \\
\hline-6679.070 & 53.125 & 58.710 & 45.740 & -0.0180 & 0.22000 & -0.23900 \\
\hline-6675.058 & 53.125 & 58.577 & 45.720 & -0.0183 & 0.21300 & -0.24100 \\
\hline-6671.047 & 53.125 & 58.503 & 45.642 & -0.0186 & 0.20700 & -0.24200 \\
\hline-6667.035 & 52.875 & 58.413 & 45.540 & -0.0189 & 0.20000 & -0.24400 \\
\hline-6663.023 & 52.875 & 58.361 & 45.352 & -0.0192 & 0.19400 & -0.24600 \\
\hline-6659.012 & 52.875 & 58.314 & 45.135 & -0.0195 & 0.19000 & -0.24900 \\
\hline-6655.000 & 52.625 & 58.271 & 44.810 & -0.0197 & 0.18700 & -0.25000 \\
\hline-6650.988 & 52.625 & 58.287 & 44.492 & -0.0200 & 0.18500 & -0.25200 \\
\hline-6646.977 & 52.375 & 58.310 & 44.157 & -0.0203 & 0.18500 & -0.25200 \\
\hline-6642.965 & 52.375 & 58.370 & 43.778 & -0.0206 & 0.18400 & -0.25400 \\
\hline-6638.953 & 52.375 & 58.451 & 43.296 & -0.0208 & 0.18400 & -0.25500 \\
\hline-6634.942 & 52.125 & 58.547 & 42.802 & -0.0211 & 0.18600 & -0.25500 \\
\hline-6630.930 & 52.125 & 58.662 & 42.315 & -0.0213 & 0.18600 & -0.25700 \\
\hline-6626.919 & 52.125 & 58.796 & 41.826 & -0.0216 & 0.18900 & -0.25500 \\
\hline-6622.907 & 51.875 & 58.926 & 41.331 & -0.0218 & 0.19000 & -0.25700 \\
\hline-6618.895 & 51.875 & 59.022 & 40.822 & -0.0221 & 0.19500 & -0.25800 \\
\hline-6614.884 & 51.875 & 59.175 & 40.413 & -0.0223 & 0.20300 & -0.25500 \\
\hline-6610.872 & 51.625 & 59.306 & 40.063 & -0.0225 & 0.20700 & -0.25600 \\
\hline-6606.860 & 51.625 & 59.435 & 39.650 & -0.0227 & 0.21100 & -0.25700 \\
\hline
\end{tabular}




\begin{tabular}{|c|c|c|c|c|c|c|}
\hline-6602.849 & 51.375 & 59.542 & 39.343 & -0.0229 & 0.21600 & -0.25700 \\
\hline-6598.837 & 51.375 & 59.599 & 38.976 & -0.0232 & 0.22200 & -0.25900 \\
\hline-6594.826 & 51.375 & 59.684 & 38.688 & -0.0234 & 0.22900 & -0.25900 \\
\hline-6590.814 & 51.125 & 59.792 & 38.420 & -0.0236 & 0.23400 & -0.26100 \\
\hline-6586.802 & 51.125 & 59.860 & 38.110 & -0.0238 & 0.24000 & -0.26000 \\
\hline-6582.791 & 51.125 & 59.936 & 37.881 & -0.0240 & 0.24700 & -0.26300 \\
\hline-6578.779 & 50.875 & 60.002 & 37.639 & -0.0241 & 0.25000 & -0.26500 \\
\hline-6574.767 & 50.875 & 60.048 & 37.452 & -0.0243 & 0.25600 & -0.26700 \\
\hline-6570.756 & 50.625 & 60.056 & 37.243 & -0.0245 & 0.26300 & -0.27000 \\
\hline-6566.744 & 50.625 & 60.107 & 37.022 & -0.0247 & 0.26700 & -0.27100 \\
\hline-6562.733 & 50.625 & 60.146 & 36.910 & -0.0248 & 0.27000 & -0.27300 \\
\hline-6558.721 & 50.375 & 60.199 & 36.665 & -0.0250 & 0.27900 & -0.27400 \\
\hline-6554.709 & 50.375 & 60.221 & 36.541 & -0.0251 & 0.28300 & -0.27600 \\
\hline-6550.698 & 50.375 & 60.267 & 36.483 & -0.0253 & 0.28400 & -0.27800 \\
\hline-6546.686 & 50.125 & 60.296 & 36.331 & -0.0254 & 0.28700 & -0.28000 \\
\hline-6542.674 & 50.125 & 60.304 & 36.282 & -0.0256 & 0.29100 & -0.28100 \\
\hline-6538.663 & 50.125 & 60.342 & 36.241 & -0.0257 & 0.29600 & -0.28500 \\
\hline-6534.651 & 49.875 & 60.354 & 36.114 & -0.0258 & 0.29600 & -0.29000 \\
\hline-6530.640 & 49.875 & 60.371 & 36.022 & -0.0260 & 0.29800 & -0.29200 \\
\hline-6526.628 & 49.625 & 60.392 & 35.943 & -0.0261 & 0.29800 & -0.29500 \\
\hline-6522.616 & 49.625 & 60.421 & 35.826 & -0.0262 & 0.29800 & -0.30000 \\
\hline-6518.605 & 49.625 & 60.419 & 35.666 & -0.0263 & 0.29800 & -0.30300 \\
\hline-6514.593 & 49.375 & 60.443 & 35.551 & -0.0264 & 0.30100 & -0.30500 \\
\hline-6510.581 & 49.375 & 60.481 & 35.459 & -0.0265 & 0.30500 & -0.30500 \\
\hline-6506.570 & 49.375 & 60.472 & 35.283 & -0.0266 & 0.30800 & -0.30600 \\
\hline-6502.558 & 49.125 & 60.480 & 35.096 & -0.0267 & 0.30500 & -0.30900 \\
\hline-6498.547 & 49.125 & 60.510 & 34.919 & -0.0268 & 0.30700 & -0.30900 \\
\hline-6494.535 & 48.875 & 60.497 & 34.821 & -0.0269 & 0.31000 & -0.31200 \\
\hline-6490.523 & 48.875 & 60.549 & 34.730 & -0.0269 & 0.31400 & -0.31400 \\
\hline-6486.512 & 48.875 & 60.523 & 34.613 & -0.0270 & 0.31500 & -0.31500 \\
\hline-6482.500 & 48.625 & 60.503 & 34.530 & -0.0271 & 0.31300 & -0.31800 \\
\hline-6478.488 & 48.625 & 60.492 & 34.473 & -0.0271 & 0.31200 & -0.32100 \\
\hline-6474.477 & 48.375 & 60.467 & 34.323 & -0.0272 & 0.31000 & -0.32100 \\
\hline-6470.465 & 48.375 & 60.454 & 34.216 & -0.0273 & 0.31100 & -0.32100 \\
\hline-6466.453 & 48.125 & 60.456 & 34.153 & -0.0275 & 0.31100 & -0.32500 \\
\hline-6462.442 & 48.125 & 60.483 & 34.034 & -0.0276 & 0.31400 & -0.32900 \\
\hline-6458.430 & 47.875 & 60.438 & 33.911 & -0.0278 & 0.31600 & -0.33000 \\
\hline-6454.419 & 47.875 & 60.412 & 33.766 & -0.0280 & 0.32000 & -0.33200 \\
\hline-6450.407 & 47.875 & 60.348 & 33.631 & -0.0282 & 0.31800 & -0.33300 \\
\hline-6446.395 & 47.625 & 60.328 & 33.577 & -0.0284 & 0.32200 & -0.33300 \\
\hline-6442.384 & 47.625 & 60.295 & 33.477 & -0.0286 & 0.31800 & -0.33900 \\
\hline-6438.372 & 47.375 & 60.220 & 33.334 & -0.0289 & 0.32100 & -0.34200 \\
\hline-6434.360 & 47.375 & 60.188 & 33.260 & -0.0292 & 0.32100 & -0.34300 \\
\hline-6430.349 & 47.125 & 60.121 & 33.249 & -0.0295 & 0.32100 & -0.34400 \\
\hline-6426.337 & 47.125 & 60.063 & 33.186 & -0.0298 & 0.32200 & -0.34400 \\
\hline-6422.326 & 46.875 & 59.993 & 33.131 & -0.0302 & 0.31900 & -0.34700 \\
\hline-6418.314 & 46.875 & 59.950 & 33.092 & -0.0305 & 0.31500 & -0.34700 \\
\hline-6414.302 & 46.625 & 59.888 & 33.004 & -0.0309 & 0.31100 & -0.35000 \\
\hline-6410.291 & 46.375 & 59.761 & 32.930 & -0.0313 & 0.30800 & -0.35200 \\
\hline-6406.279 & 46.375 & 59.612 & 32.873 & -0.0317 & 0.30600 & -0.35600 \\
\hline-6402.267 & 46.125 & 59.451 & 32.836 & -0.0322 & 0.30300 & -0.35700 \\
\hline
\end{tabular}




\begin{tabular}{|c|c|c|c|c|c|c|}
\hline-6398.256 & 46.125 & 59.352 & 32.807 & -0.0326 & 0.30000 & -0.36000 \\
\hline-6394.244 & 45.875 & 59.231 & 32.724 & -0.0331 & 0.29900 & -0.36300 \\
\hline-6390.233 & 45.875 & 59.107 & 32.700 & -0.0336 & 0.29800 & -0.36200 \\
\hline-6386.221 & 45.625 & 58.964 & 32.670 & -0.0341 & 0.29400 & -0.36500 \\
\hline-6382.209 & 45.625 & 58.805 & 32.596 & -0.0346 & 0.28800 & -0.36800 \\
\hline-6378.198 & 45.375 & 58.685 & 32.587 & -0.0352 & 0.28600 & -0.36900 \\
\hline-6374.186 & 45.125 & 58.510 & 32.625 & -0.0358 & 0.28000 & -0.37900 \\
\hline-6370.174 & 45.125 & 58.258 & 32.597 & -0.0364 & 0.27700 & -0.38100 \\
\hline-6366.163 & 44.875 & 58.049 & 32.609 & -0.0370 & 0.27300 & -0.38100 \\
\hline-6362.151 & 44.625 & 57.900 & 32.636 & -0.0376 & 0.27000 & -0.38300 \\
\hline-6358.140 & 44.625 & 57.656 & 32.601 & -0.0383 & 0.26200 & -0.38600 \\
\hline-6354.128 & 44.375 & 57.425 & 32.703 & -0.0389 & 0.26000 & -0.38600 \\
\hline-6350.116 & 44.375 & 57.185 & 32.804 & -0.0396 & 0.25500 & -0.38800 \\
\hline-6346.105 & 44.125 & 56.924 & 32.847 & -0.0402 & 0.24900 & -0.39100 \\
\hline-6342.093 & 43.875 & 56.614 & 32.921 & -0.0405 & 0.24300 & -0.39200 \\
\hline-6338.081 & 43.875 & 56.340 & 32.985 & -0.0407 & 0.23900 & -0.38800 \\
\hline-6334.070 & 43.625 & 56.014 & 33.085 & -0.0406 & 0.23400 & -0.38400 \\
\hline-6330.058 & 43.375 & 55.725 & 33.232 & -0.0403 & 0.23000 & -0.38200 \\
\hline-6326.047 & 43.375 & 55.335 & 33.333 & -0.0398 & 0.22400 & -0.38300 \\
\hline-6322.035 & 43.125 & 54.977 & 33.447 & -0.0391 & 0.22100 & -0.37800 \\
\hline-6318.023 & 43.125 & 54.593 & 33.602 & -0.0381 & 0.21600 & -0.37500 \\
\hline-6314.012 & 42.875 & 54.218 & 33.755 & -0.0369 & 0.21300 & -0.36600 \\
\hline-6310.000 & 42.625 & 53.932 & 33.948 & -0.0355 & 0.21000 & -0.35600 \\
\hline-6305.988 & 42.625 & 53.610 & 34.230 & -0.0339 & 0.20800 & -0.34400 \\
\hline-6301.977 & 42.375 & 53.283 & 34.429 & -0.0320 & 0.20300 & -0.33300 \\
\hline-6297.965 & 42.375 & 52.837 & 34.625 & -0.0300 & 0.19800 & -0.31600 \\
\hline-6293.953 & 42.375 & 52.454 & 34.863 & -0.0277 & 0.19200 & -0.30400 \\
\hline-6289.942 & 42.125 & 51.963 & 35.104 & -0.0252 & 0.18800 & -0.29000 \\
\hline-6285.930 & 42.125 & 51.535 & 35.333 & -0.0225 & 0.18500 & -0.27600 \\
\hline-6281.919 & 42.125 & 51.070 & 35.563 & -0.0197 & 0.17800 & -0.26500 \\
\hline-6277.907 & 41.875 & 50.631 & 35.749 & -0.0171 & 0.17400 & -0.25200 \\
\hline-6273.895 & 41.875 & 50.129 & 35.946 & -0.0147 & 0.16700 & -0.24200 \\
\hline-6269.884 & 41.875 & 49.686 & 36.157 & -0.0125 & 0.16200 & -0.22700 \\
\hline-6265.872 & 41.875 & 49.261 & 36.355 & -0.0104 & 0.15700 & -0.21600 \\
\hline-6261.860 & 41.875 & 48.795 & 36.528 & -0.00850 & 0.14900 & -0.20500 \\
\hline-6257.849 & 41.875 & 48.390 & 36.698 & -0.00670 & 0.14200 & -0.19800 \\
\hline-6253.837 & 41.875 & 48.000 & 36.860 & -0.00520 & 0.13700 & -0.18800 \\
\hline-6249.826 & 41.625 & 47.599 & 36.985 & -0.00380 & 0.13100 & -0.17700 \\
\hline-6245.814 & 41.625 & 47.278 & 37.138 & -0.00250 & 0.12600 & -0.16600 \\
\hline-6241.802 & 41.625 & 46.930 & 37.276 & -0.00150 & 0.12100 & -0.15600 \\
\hline-6237.791 & 41.625 & 46.640 & 37.415 & -0.000600 & 0.11800 & -0.14600 \\
\hline-6233.779 & 41.625 & 46.418 & 37.522 & $1.00 \mathrm{e}-04$ & 0.11400 & -0.13600 \\
\hline-6229.767 & 41.625 & 46.226 & 37.669 & 0.000700 & 0.10900 & -0.12800 \\
\hline-6225.756 & 41.625 & 46.045 & 37.757 & 0.00110 & 0.10700 & -0.11900 \\
\hline-6221.744 & 41.625 & 45.886 & 37.883 & 0.00130 & 0.10300 & -0.11100 \\
\hline-6217.733 & 41.625 & 45.696 & 38.009 & 0.00140 & 0.10000 & -0.10400 \\
\hline-6213.721 & 41.625 & 45.533 & 38.101 & 0.00150 & 0.096000 & -0.097000 \\
\hline-6209.709 & 41.625 & 45.401 & 38.201 & 0.00170 & 0.093000 & -0.089000 \\
\hline-6205.698 & 41.625 & 45.291 & 38.268 & 0.00180 & 0.091000 & -0.082000 \\
\hline-6201.686 & 41.625 & 45.182 & 38.357 & 0.00190 & 0.086000 & -0.077000 \\
\hline-6197.674 & 41.625 & 45.092 & 38.442 & 0.00200 & 0.083000 & -0.072000 \\
\hline
\end{tabular}




\begin{tabular}{|c|c|c|c|c|c|c|}
\hline-6193.663 & 41.625 & 45.025 & 38.525 & 0.00210 & 0.080000 & -0.068000 \\
\hline-6189.651 & 41.625 & 44.948 & 38.578 & 0.00220 & 0.077000 & -0.065000 \\
\hline-6185.640 & 41.625 & 44.894 & 38.636 & 0.00240 & 0.074000 & -0.061000 \\
\hline-6181.628 & 41.625 & 44.852 & 38.688 & 0.00250 & 0.072000 & -0.058000 \\
\hline-6177.616 & 41.625 & 44.801 & 38.756 & 0.00260 & 0.070000 & -0.055000 \\
\hline-6173.605 & 41.625 & 44.762 & 38.822 & 0.00270 & 0.068000 & -0.053000 \\
\hline-6169.593 & 41.875 & 44.710 & 38.865 & 0.00280 & 0.067000 & -0.049000 \\
\hline-6165.581 & 41.875 & 44.689 & 38.911 & 0.00290 & 0.065000 & -0.047000 \\
\hline-6161.570 & 41.875 & 44.675 & 38.967 & 0.00300 & 0.064000 & -0.045000 \\
\hline-6157.558 & 41.875 & 44.650 & 39.047 & 0.00310 & 0.063000 & -0.044000 \\
\hline-6153.547 & 41.875 & 44.623 & 39.101 & 0.00320 & 0.062000 & -0.042000 \\
\hline-6149.535 & 41.875 & 44.615 & 39.156 & 0.00330 & 0.061000 & -0.040000 \\
\hline-6145.523 & 41.875 & 44.625 & 39.211 & 0.00340 & 0.061000 & -0.039000 \\
\hline-6141.512 & 41.875 & 44.646 & 39.255 & 0.00350 & 0.061000 & -0.037000 \\
\hline-6137.500 & 41.875 & 44.662 & 39.306 & 0.00360 & 0.060000 & -0.036000 \\
\hline-6133.488 & 41.875 & 44.687 & 39.344 & 0.00370 & 0.060000 & -0.035000 \\
\hline-6129.477 & 41.875 & 44.697 & 39.391 & 0.00370 & 0.061000 & -0.035000 \\
\hline-6125.465 & 41.875 & 44.718 & 39.436 & 0.00380 & 0.061000 & -0.034000 \\
\hline-6121.453 & 41.875 & 44.746 & 39.482 & 0.00390 & 0.061000 & -0.033000 \\
\hline-6117.442 & 41.875 & 44.789 & 39.524 & 0.00400 & 0.061000 & -0.033000 \\
\hline-6113.430 & 41.875 & 44.839 & 39.567 & 0.00410 & 0.062000 & -0.032000 \\
\hline-6109.419 & 41.875 & 44.896 & 39.603 & 0.00420 & 0.062000 & -0.032000 \\
\hline-6105.407 & 41.875 & 44.973 & 39.653 & 0.00420 & 0.062000 & -0.032000 \\
\hline-6101.395 & 41.875 & 45.046 & 39.687 & 0.00430 & 0.062000 & -0.032000 \\
\hline-6097.384 & 41.875 & 45.114 & 39.729 & 0.00440 & 0.063000 & -0.032000 \\
\hline-6093.372 & 41.875 & 45.189 & 39.779 & 0.00450 & 0.064000 & -0.032000 \\
\hline-6089.360 & 42.125 & 45.267 & 39.818 & 0.00450 & 0.065000 & -0.032000 \\
\hline-6085.349 & 42.125 & 45.372 & 39.855 & 0.00460 & 0.066000 & -0.032000 \\
\hline-6081.337 & 42.125 & 45.471 & 39.893 & 0.00470 & 0.067000 & -0.032000 \\
\hline-6077.326 & 42.125 & 45.575 & 39.928 & 0.00470 & 0.068000 & -0.033000 \\
\hline-6073.314 & 42.125 & 45.706 & 39.968 & 0.00480 & 0.070000 & -0.033000 \\
\hline-6069.302 & 42.125 & 45.830 & 40.008 & 0.00490 & 0.072000 & -0.034000 \\
\hline-6065.291 & 42.125 & 45.967 & 40.056 & 0.00490 & 0.073000 & -0.035000 \\
\hline-6061.279 & 42.125 & 46.070 & 40.103 & 0.00500 & 0.075000 & -0.036000 \\
\hline-6057.267 & 42.125 & 46.183 & 40.144 & 0.00510 & 0.077000 & -0.037000 \\
\hline-6053.256 & 42.125 & 46.282 & 40.191 & 0.00510 & 0.079000 & -0.038000 \\
\hline-6049.244 & 42.125 & 46.440 & 40.226 & 0.00520 & 0.081000 & -0.039000 \\
\hline-6045.233 & 42.125 & 46.581 & 40.263 & 0.00520 & 0.083000 & -0.041000 \\
\hline-6041.221 & 42.125 & 46.689 & 40.296 & 0.00530 & 0.085000 & -0.043000 \\
\hline-6037.209 & 42.125 & 46.811 & 40.341 & 0.00530 & 0.087000 & -0.045000 \\
\hline-6033.198 & 42.375 & 46.963 & 40.376 & 0.00540 & 0.091000 & -0.047000 \\
\hline-6029.186 & 42.375 & 47.095 & 40.410 & 0.00540 & 0.094000 & -0.050000 \\
\hline-6025.174 & 42.375 & 47.231 & 40.441 & 0.00550 & 0.095000 & -0.053000 \\
\hline-6021.163 & 42.375 & 47.356 & 40.473 & 0.00550 & 0.098000 & -0.056000 \\
\hline-6017.151 & 42.375 & 47.464 & 40.500 & 0.00560 & 0.099000 & -0.061000 \\
\hline-6013.140 & 42.375 & 47.553 & 40.523 & 0.00560 & 0.10200 & -0.065000 \\
\hline-6009.128 & 42.375 & 47.645 & 40.552 & 0.00570 & 0.10400 & -0.069000 \\
\hline-6005.116 & 42.375 & 47.698 & 40.573 & 0.00570 & 0.10600 & -0.074000 \\
\hline-6001.105 & 42.375 & 47.767 & 40.596 & 0.00570 & 0.10800 & -0.080000 \\
\hline-5997.093 & 42.375 & 47.801 & 40.602 & 0.00580 & 0.11200 & -0.084000 \\
\hline-5993.081 & 42.625 & 47.818 & 40.600 & 0.00580 & 0.11600 & $-0.0880 c$ \\
\hline
\end{tabular}




\begin{tabular}{|c|c|c|c|c|c|c|}
\hline-5989.070 & 42.625 & 47.853 & 40.609 & 0.00580 & 0.11900 & -0.093000 \\
\hline-5985.058 & 42.625 & 47.892 & 40.618 & 0.00590 & 0.12400 & -0.098000 \\
\hline-5981.047 & 42.625 & 47.930 & 40.627 & 0.00590 & 0.12900 & -0.10300 \\
\hline-5977.035 & 42.625 & 48.004 & 40.641 & 0.00590 & 0.13300 & -0.10800 \\
\hline-5973.023 & 42.625 & 48.072 & 40.648 & 0.00590 & 0.13900 & -0.11400 \\
\hline-5969.012 & 42.625 & 48.162 & 40.635 & 0.00600 & 0.14400 & -0.12000 \\
\hline-5965.000 & 42.875 & 48.260 & 40.622 & 0.00600 & 0.15000 & -0.12600 \\
\hline-5960.988 & 42.875 & 48.398 & 40.609 & 0.00640 & 0.15700 & -0.13000 \\
\hline-5956.977 & 42.875 & 48.512 & 40.593 & 0.00730 & 0.16600 & -0.13300 \\
\hline-5952.965 & 42.875 & 48.659 & 40.594 & 0.00880 & 0.17400 & -0.13900 \\
\hline-5948.953 & 42.875 & 48.848 & 40.556 & 0.0108 & 0.18400 & -0.14400 \\
\hline-5944.942 & 43.125 & 49.060 & 40.530 & 0.0134 & 0.19200 & -0.15200 \\
\hline-5940.930 & 43.125 & 49.308 & 40.484 & 0.0166 & 0.20300 & -0.15800 \\
\hline-5936.919 & 43.125 & 49.561 & 40.447 & 0.0204 & 0.21400 & -0.16400 \\
\hline-5932.907 & 43.375 & 49.811 & 40.420 & 0.0247 & 0.22700 & -0.17100 \\
\hline-5928.895 & 43.375 & 50.111 & 40.386 & 0.0297 & 0.24100 & -0.18200 \\
\hline-5924.884 & 43.375 & 50.428 & 40.326 & 0.0352 & 0.25500 & -0.19100 \\
\hline-5920.872 & 43.625 & 50.788 & 40.241 & 0.0413 & 0.26900 & -0.20000 \\
\hline-5916.860 & 43.625 & 51.175 & 40.121 & 0.0479 & 0.29000 & -0.20900 \\
\hline-5912.849 & 43.875 & 51.615 & 39.957 & 0.0552 & 0.31300 & -0.21600 \\
\hline-5908.837 & 44.125 & 52.086 & 39.800 & 0.0630 & 0.33800 & -0.22900 \\
\hline-5904.826 & 44.375 & 52.638 & 39.575 & 0.0714 & 0.37100 & -0.23500 \\
\hline-5900.814 & 44.625 & 53.284 & 39.387 & 0.0804 & 0.39700 & -0.24500 \\
\hline-5896.802 & 44.875 & 54.017 & 39.179 & 0.0898 & 0.42600 & -0.25400 \\
\hline-5892.791 & 45.125 & 54.838 & 38.914 & 0.0991 & 0.46000 & -0.26400 \\
\hline-5888.779 & 45.625 & 55.702 & 38.592 & 0.108 & 0.49000 & -0.27200 \\
\hline-5884.767 & 46.125 & 56.698 & 38.230 & 0.115 & 0.52600 & -0.27700 \\
\hline-5880.756 & 46.625 & 57.749 & 37.887 & 0.122 & 0.56000 & -0.27800 \\
\hline-5876.744 & 47.125 & 58.878 & 37.573 & 0.128 & 0.58600 & -0.28300 \\
\hline-5872.733 & 47.625 & 59.939 & 37.330 & 0.133 & 0.60500 & -0.28600 \\
\hline-5868.721 & 48.125 & 60.810 & 37.102 & 0.137 & 0.62200 & -0.28500 \\
\hline-5864.709 & 48.625 & 61.620 & 36.905 & 0.140 & 0.64500 & -0.27800 \\
\hline-5860.698 & 49.375 & 62.237 & 36.776 & 0.142 & 0.68300 & -0.26900 \\
\hline-5856.686 & 49.875 & 62.675 & 36.712 & 0.144 & 0.73200 & -0.26400 \\
\hline-5852.674 & 50.375 & 63.059 & 36.693 & 0.144 & 0.80300 & -0.25800 \\
\hline-5848.663 & 51.125 & 63.311 & 36.868 & 0.144 & 0.87700 & -0.25600 \\
\hline-5844.651 & 51.625 & 63.450 & 37.077 & 0.143 & 0.96400 & -0.25600 \\
\hline-5840.640 & 52.375 & 63.536 & 37.350 & 0.140 & 1.0280 & -0.26000 \\
\hline-5836.628 & 53.125 & 63.584 & 37.737 & 0.137 & 1.0970 & -0.27000 \\
\hline-5832.616 & 53.625 & 63.516 & 38.155 & 0.133 & 1.1150 & -0.28000 \\
\hline-5828.605 & 54.375 & 63.490 & 38.685 & 0.126 & 1.1320 & -0.29400 \\
\hline-5824.593 & 54.875 & 63.517 & 39.243 & 0.113 & 1.0980 & -0.31600 \\
\hline-5820.581 & 55.125 & 63.530 & 39.687 & 0.0950 & 1.0250 & -0.34400 \\
\hline-5816.570 & 55.375 & 63.545 & 40.134 & 0.0714 & 0.95500 & -0.38000 \\
\hline-5812.558 & 55.375 & 63.483 & 40.606 & 0.0424 & 0.89300 & -0.42100 \\
\hline-5808.547 & 55.375 & 63.356 & 41.118 & 0.00780 & 0.82800 & -0.47000 \\
\hline-5804.535 & 55.375 & 63.152 & 41.667 & -0.0322 & 0.75200 & -0.52700 \\
\hline-5800.523 & 55.125 & 62.840 & 42.353 & -0.0766 & 0.68400 & -0.60200 \\
\hline-5796.512 & 54.625 & 62.445 & 43.062 & -0.119 & 0.61500 & -0.70000 \\
\hline-5792.500 & 54.125 & 61.986 & 43.325 & -0.155 & 0.55800 & -0.81200 \\
\hline-5788.488 & 53.375 & 61.511 & 43.476 & -0.183 & 0.49700 & -0.88600 \\
\hline
\end{tabular}




\begin{tabular}{|c|c|c|c|c|c|c|}
\hline-5784.477 & 52.625 & 61.057 & 43.536 & -0.202 & 0.44100 & -0.90400 \\
\hline-5780.465 & 51.875 & 60.565 & 43.481 & -0.213 & 0.39500 & -0.90500 \\
\hline-5776.453 & 51.125 & 60.059 & 43.354 & -0.216 & 0.35500 & -0.89900 \\
\hline-5772.442 & 50.375 & 59.467 & 43.180 & -0.210 & 0.31700 & -0.86100 \\
\hline-5768.430 & 49.625 & 58.863 & 42.938 & -0.197 & 0.28400 & -0.80500 \\
\hline-5764.419 & 48.875 & 58.184 & 42.674 & -0.180 & 0.25800 & -0.75000 \\
\hline-5760.407 & 48.125 & 57.415 & 42.388 & -0.164 & 0.22600 & -0.69400 \\
\hline-5756.395 & 47.375 & 56.611 & 42.095 & -0.148 & 0.20900 & -0.62800 \\
\hline-5752.384 & 46.875 & 55.744 & 41.887 & -0.133 & 0.18600 & -0.57300 \\
\hline-5748.372 & 46.125 & 54.894 & 41.754 & -0.119 & 0.16300 & -0.51600 \\
\hline-5744.360 & 45.875 & 53.955 & 41.652 & -0.106 & 0.14600 & -0.46700 \\
\hline-5740.349 & 45.375 & 53.091 & 41.501 & -0.0939 & 0.12900 & -0.42000 \\
\hline-5736.337 & 45.125 & 52.346 & 41.401 & -0.0827 & 0.11500 & -0.37400 \\
\hline-5732.326 & 44.625 & 51.688 & 41.310 & -0.0724 & 0.11300 & -0.33600 \\
\hline-5728.314 & 44.375 & 51.089 & 41.202 & -0.0630 & 0.11300 & -0.29900 \\
\hline-5724.302 & 44.375 & 50.581 & 41.056 & -0.0545 & 0.11300 & -0.26500 \\
\hline-5720.291 & 44.125 & 50.129 & 40.926 & -0.0469 & 0.11200 & -0.23700 \\
\hline-5716.279 & 43.875 & 49.677 & 40.854 & -0.0403 & 0.10900 & -0.21400 \\
\hline-5712.267 & 43.875 & 49.162 & 40.807 & -0.0345 & 0.10500 & -0.19600 \\
\hline-5708.256 & 43.625 & 48.646 & 40.781 & -0.0297 & 0.10300 & -0.18300 \\
\hline-5704.244 & 43.625 & 48.180 & 40.715 & -0.0257 & 0.10500 & -0.17000 \\
\hline-5700.233 & 43.375 & 47.768 & 40.661 & -0.0225 & 0.10300 & -0.16000 \\
\hline-5696.221 & 43.375 & 47.414 & 40.567 & -0.0201 & 0.10200 & -0.15400 \\
\hline-5692.209 & 43.375 & 47.080 & 40.453 & -0.0183 & 0.10100 & -0.15000 \\
\hline-5688.198 & 43.125 & 46.774 & 40.306 & -0.0172 & 0.10000 & -0.14700 \\
\hline-5684.186 & 43.125 & 46.547 & 40.122 & -0.0169 & 0.10000 & -0.14500 \\
\hline-5680.174 & 43.125 & 46.386 & 39.888 & -0.0172 & 0.10200 & -0.14400 \\
\hline-5676.163 & 42.875 & 46.277 & 39.627 & -0.0181 & 0.10300 & -0.14400 \\
\hline-5672.151 & 42.875 & 46.264 & 39.368 & -0.0198 & 0.10500 & -0.14700 \\
\hline-5668.140 & 42.625 & 46.214 & 39.084 & -0.0222 & 0.10600 & -0.15200 \\
\hline-5664.128 & 42.625 & 46.187 & 38.775 & -0.0253 & 0.10800 & -0.16100 \\
\hline-5660.116 & 42.625 & 46.119 & 38.449 & -0.0290 & 0.11000 & -0.17200 \\
\hline-5656.105 & 42.375 & 46.089 & 38.093 & -0.0334 & 0.11400 & -0.18400 \\
\hline-5652.093 & 42.375 & 46.056 & 37.720 & -0.0386 & 0.11900 & -0.19800 \\
\hline-5648.081 & 42.125 & 46.032 & 37.334 & -0.0444 & 0.12500 & -0.21800 \\
\hline-5644.070 & 41.875 & 46.041 & 36.924 & -0.0509 & 0.13100 & -0.24100 \\
\hline-5640.058 & 41.875 & 46.113 & 36.480 & -0.0583 & 0.14100 & -0.27100 \\
\hline-5636.047 & 41.625 & 46.213 & 36.014 & -0.0676 & 0.15900 & -0.31100 \\
\hline-5632.035 & 41.375 & 46.354 & 35.480 & -0.0798 & 0.17600 & -0.36100 \\
\hline-5628.023 & 41.125 & 46.640 & 34.877 & -0.0951 & 0.19400 & -0.41900 \\
\hline-5624.012 & 40.625 & 46.988 & 34.145 & -0.113 & 0.21300 & -0.49900 \\
\hline-5620.000 & 40.125 & 47.351 & 33.232 & -0.135 & 0.22900 & -0.59800 \\
\hline-5615.988 & 39.375 & 47.563 & 32.069 & -0.160 & 0.24500 & -0.72200 \\
\hline-5611.977 & 38.625 & 47.697 & 30.697 & -0.187 & 0.27800 & -0.87300 \\
\hline-5607.965 & 37.625 & 47.473 & 29.218 & -0.216 & 0.34300 & -1.0520 \\
\hline-5603.953 & 36.625 & 46.894 & 28.176 & -0.233 & 0.46600 & -1.2070 \\
\hline-5599.942 & 35.875 & 46.267 & 27.641 & -0.227 & 0.64400 & -1.3370 \\
\hline-5595.930 & 35.125 & 45.474 & 27.381 & -0.195 & 0.97800 & -1.3450 \\
\hline-5591.919 & 34.625 & 45.758 & 27.070 & -0.138 & 1.4520 & -1.2530 \\
\hline-5587.907 & 34.375 & 47.391 & 26.811 & -0.0543 & 1.8440 & -1.1790 \\
\hline-5583.895 & 34.375 & 48.807 & 26.800 & 0.0547 & 2.2040 & -1.0930 \\
\hline
\end{tabular}




\begin{tabular}{|c|c|c|c|c|c|c|}
\hline-5579.884 & 34.625 & 49.845 & 26.840 & 0.189 & 2.4040 & -0.99900 \\
\hline-5575.872 & 35.125 & 50.553 & 26.997 & 0.341 & 2.5310 & -0.88400 \\
\hline-5571.860 & 36.375 & 51.164 & 27.295 & 0.465 & 2.5990 & -0.78900 \\
\hline-5567.849 & 38.625 & 51.505 & 27.623 & 0.515 & 2.5230 & -0.68400 \\
\hline-5563.837 & 41.875 & 51.701 & 28.154 & 0.476 & 2.4620 & -0.61200 \\
\hline-5559.826 & 43.875 & 51.753 & 28.786 & 0.352 & 2.2450 & -0.55000 \\
\hline-5555.814 & 44.375 & 51.721 & 29.514 & 0.199 & 1.9840 & -0.49200 \\
\hline-5551.802 & 44.625 & 51.567 & 30.290 & 0.0771 & 1.7840 & -0.45600 \\
\hline-5547.791 & 44.875 & 51.438 & 31.190 & -0.00340 & 1.5950 & -0.43200 \\
\hline-5543.779 & 44.625 & 51.267 & 32.233 & -0.0454 & 1.3410 & -0.42800 \\
\hline-5539.767 & 44.375 & 50.993 & 33.560 & -0.0657 & 1.1110 & -0.42500 \\
\hline-5535.756 & 44.125 & 50.715 & 36.118 & -0.0808 & 0.88500 & -0.42200 \\
\hline-5531.744 & 43.875 & 50.445 & 37.238 & -0.0941 & 0.70600 & -0.42100 \\
\hline-5527.733 & 43.375 & 50.084 & 37.327 & -0.106 & 0.53000 & -0.41200 \\
\hline-5523.721 & 42.875 & 49.543 & 36.948 & -0.115 & 0.39100 & -0.40400 \\
\hline-5519.709 & 42.375 & 48.938 & 36.427 & -0.123 & 0.28900 & -0.39400 \\
\hline-5515.698 & 41.875 & 48.231 & 35.886 & -0.130 & 0.18500 & -0.38300 \\
\hline-5511.686 & 41.125 & 47.554 & 35.355 & -0.134 & -0.022000 & -0.37200 \\
\hline-5507.674 & 40.625 & 46.859 & 34.831 & -0.137 & -0.031000 & -0.36100 \\
\hline-5503.663 & 40.125 & 46.095 & 34.335 & -0.140 & -0.031000 & -0.34800 \\
\hline-5499.651 & 39.625 & 45.354 & 33.893 & -0.141 & -0.030000 & -0.33500 \\
\hline-5495.640 & 39.125 & 44.602 & 33.504 & -0.142 & -0.027000 & -0.32100 \\
\hline-5491.628 & 38.625 & 43.828 & 33.173 & -0.142 & -0.025000 & -0.30700 \\
\hline-5487.616 & 37.875 & 43.083 & 32.890 & -0.142 & -0.022000 & -0.29600 \\
\hline-5483.605 & 37.375 & 42.330 & 32.653 & -0.140 & -0.020000 & -0.28300 \\
\hline-5479.593 & 36.875 & 41.604 & 32.441 & -0.138 & -0.018000 & -0.27200 \\
\hline-5475.581 & 36.375 & 40.902 & 32.238 & -0.135 & -0.016000 & -0.26000 \\
\hline-5471.570 & 35.875 & 40.232 & 32.034 & -0.131 & -0.014000 & -0.25000 \\
\hline-5467.558 & 35.375 & 39.585 & 31.830 & -0.127 & -0.012000 & -0.24000 \\
\hline-5463.547 & 34.875 & 38.975 & 31.605 & -0.122 & -0.0100000 & -0.23100 \\
\hline-5459.535 & 34.625 & 38.389 & 31.338 & -0.116 & -0.0070000 & -0.22300 \\
\hline-5455.523 & 34.125 & 37.834 & 31.023 & -0.109 & -0.0050000 & -0.21700 \\
\hline-5451.512 & 33.625 & 37.301 & 30.647 & -0.101 & -0.0010000 & -0.21000 \\
\hline-5447.500 & 33.375 & 36.796 & 30.234 & -0.0930 & 0.0060000 & -0.20500 \\
\hline-5443.488 & 32.875 & 36.301 & 29.789 & -0.0845 & 0.017000 & -0.20100 \\
\hline-5439.477 & 32.625 & 35.831 & 29.414 & -0.0761 & 0.035000 & -0.19600 \\
\hline-5435.465 & 32.375 & 35.379 & 29.099 & -0.0681 & 0.044000 & -0.19100 \\
\hline-5431.453 & 32.125 & 34.959 & 28.861 & -0.0603 & 0.049000 & -0.18500 \\
\hline-5427.442 & 31.875 & 34.558 & 28.694 & -0.0527 & 0.052000 & -0.17800 \\
\hline-5423.430 & 31.625 & 34.181 & 28.564 & -0.0454 & 0.055000 & -0.17200 \\
\hline-5419.419 & 31.375 & 33.838 & 28.468 & -0.0383 & 0.058000 & -0.16400 \\
\hline-5415.407 & 31.125 & 33.521 & 28.400 & -0.0315 & 0.061000 & -0.15700 \\
\hline-5411.395 & 30.875 & 33.230 & 28.324 & -0.0249 & 0.064000 & -0.15000 \\
\hline-5407.384 & 30.625 & 32.989 & 28.269 & -0.0186 & 0.066000 & -0.14300 \\
\hline-5403.372 & 30.625 & 32.787 & 28.225 & -0.0126 & 0.069000 & -0.13600 \\
\hline-5399.360 & 30.625 & 32.626 & 28.197 & -0.00680 & 0.072000 & -0.12900 \\
\hline-5395.349 & 30.375 & 32.509 & 28.174 & -0.00120 & 0.075000 & -0.12200 \\
\hline-5391.337 & 30.625 & 32.443 & 28.167 & 0.00410 & 0.079000 & -0.11500 \\
\hline-5387.326 & 30.625 & 32.432 & 28.186 & 0.00910 & 0.083000 & -0.10900 \\
\hline-5383.314 & 30.625 & 32.495 & 28.223 & 0.0139 & 0.087000 & -0.10200 \\
\hline-5379.302 & 30.625 & 32.608 & 28.251 & 0.0184 & 0.092000 & -0.096000 \\
\hline
\end{tabular}




\begin{tabular}{|c|c|c|c|c|c|c|}
\hline-5375.291 & 30.875 & 32.764 & 28.299 & 0.0227 & 0.098000 & -0.090000 \\
\hline-5371.279 & 30.875 & 32.948 & 28.355 & 0.0268 & 0.10400 & -0.082000 \\
\hline-5367.267 & 31.125 & 33.161 & 28.427 & 0.0305 & 0.11000 & -0.073000 \\
\hline-5363.256 & 31.125 & 33.398 & 28.507 & 0.0341 & 0.11800 & -0.065000 \\
\hline-5359.244 & 31.375 & 33.639 & 28.608 & 0.0374 & 0.12500 & -0.058000 \\
\hline-5355.233 & 31.625 & 33.903 & 28.722 & 0.0404 & 0.13300 & -0.052000 \\
\hline-5351.221 & 31.875 & 34.193 & 28.860 & 0.0432 & 0.14200 & -0.047000 \\
\hline-5347.209 & 31.875 & 34.500 & 29.000 & 0.0457 & 0.14700 & -0.043000 \\
\hline-5343.198 & 32.125 & 34.821 & 29.158 & 0.0479 & 0.15300 & -0.039000 \\
\hline-5339.186 & 32.375 & 35.171 & 29.321 & 0.0500 & 0.15400 & -0.035000 \\
\hline-5335.174 & 32.625 & 35.538 & 29.486 & 0.0517 & 0.15400 & -0.032000 \\
\hline-5331.163 & 32.875 & 35.918 & 29.657 & 0.0532 & 0.15200 & -0.030000 \\
\hline-5327.151 & 33.125 & 36.298 & 29.832 & 0.0545 & 0.14900 & -0.029000 \\
\hline-5323.140 & 33.375 & 36.652 & 30.017 & 0.0555 & 0.14700 & -0.027000 \\
\hline-5319.128 & 33.625 & 36.968 & 30.195 & 0.0562 & 0.14200 & -0.026000 \\
\hline-5315.116 & 33.875 & 37.253 & 30.396 & 0.0568 & 0.13800 & -0.025000 \\
\hline-5311.105 & 34.125 & 37.494 & 30.595 & 0.0571 & 0.13300 & -0.025000 \\
\hline-5307.093 & 34.375 & 37.698 & 30.805 & 0.0575 & 0.12900 & -0.026000 \\
\hline-5303.081 & 34.625 & 37.885 & 31.011 & 0.0578 & 0.12600 & -0.028000 \\
\hline-5299.070 & 34.875 & 38.047 & 31.218 & 0.0581 & 0.12300 & -0.031000 \\
\hline-5295.058 & 35.125 & 38.228 & 31.436 & 0.0583 & 0.12100 & -0.036000 \\
\hline-5291.047 & 35.125 & 38.411 & 31.649 & 0.0586 & 0.11900 & -0.040000 \\
\hline-5287.035 & 35.375 & 38.594 & 31.877 & 0.0588 & 0.11700 & -0.046000 \\
\hline-5283.023 & 35.625 & 38.785 & 32.089 & 0.0589 & 0.11600 & -0.052000 \\
\hline-5279.012 & 35.875 & 38.977 & 32.300 & 0.0591 & 0.11500 & -0.057000 \\
\hline-5275.000 & 36.125 & 39.195 & 32.491 & 0.0592 & 0.11400 & -0.061000 \\
\hline-5270.988 & 36.375 & 39.414 & 32.693 & 0.0593 & 0.11400 & -0.065000 \\
\hline-5266.977 & 36.625 & 39.636 & 32.871 & 0.0594 & 0.11400 & -0.068000 \\
\hline-5262.965 & 36.875 & 39.853 & 33.020 & 0.0594 & 0.11500 & -0.069000 \\
\hline-5258.953 & 36.875 & 40.087 & 33.171 & 0.0594 & 0.11600 & -0.072000 \\
\hline-5254.942 & 37.125 & 40.338 & 33.309 & 0.0594 & 0.11600 & -0.074000 \\
\hline-5250.930 & 37.375 & 40.599 & 33.403 & 0.0594 & 0.11800 & -0.074000 \\
\hline-5246.919 & 37.625 & 40.869 & 33.468 & 0.0593 & 0.11900 & -0.075000 \\
\hline-5242.907 & 37.875 & 41.143 & 33.520 & 0.0593 & 0.12100 & -0.076000 \\
\hline-5238.895 & 38.125 & 41.425 & 33.551 & 0.0591 & 0.12300 & -0.077000 \\
\hline-5234.884 & 38.375 & 41.712 & 33.583 & 0.0590 & 0.12600 & -0.076000 \\
\hline-5230.872 & 38.625 & 42.011 & 33.597 & 0.0588 & 0.12800 & -0.076000 \\
\hline-5226.860 & 38.875 & 42.304 & 33.608 & 0.0586 & 0.13200 & -0.075000 \\
\hline-5222.849 & 39.125 & 42.613 & 33.601 & 0.0584 & 0.13700 & -0.076000 \\
\hline-5218.837 & 39.375 & 42.937 & 33.622 & 0.0582 & 0.14100 & -0.076000 \\
\hline-5214.826 & 39.375 & 43.262 & 33.614 & 0.0579 & 0.14600 & -0.077000 \\
\hline-5210.814 & 39.625 & 43.578 & 33.634 & 0.0576 & 0.15000 & -0.079000 \\
\hline-5206.802 & 39.875 & 43.906 & 33.692 & 0.0573 & 0.15400 & -0.083000 \\
\hline-5202.791 & 40.125 & 44.233 & 33.720 & 0.0569 & 0.15800 & -0.089000 \\
\hline-5198.779 & 40.375 & 44.570 & 33.739 & 0.0566 & 0.16400 & -0.093000 \\
\hline-5194.767 & 40.625 & 44.893 & 33.741 & 0.0562 & 0.16700 & -0.10000 \\
\hline-5190.756 & 40.875 & 45.221 & 33.713 & 0.0557 & 0.17100 & -0.10900 \\
\hline-5186.744 & 41.125 & 45.545 & 33.728 & 0.0553 & 0.17500 & -0.11800 \\
\hline-5182.733 & 41.375 & 45.851 & 33.711 & 0.0548 & 0.18000 & -0.12900 \\
\hline-5178.721 & 41.625 & 46.179 & 33.671 & 0.0543 & 0.18400 & -0.14100 \\
\hline-5174.709 & 41.875 & 46.517 & 33.598 & 0.0539 & 0.18900 & -0.1530 \\
\hline
\end{tabular}




\begin{tabular}{|c|c|c|c|c|c|c|}
\hline-5170.698 & 42.125 & 46.849 & 33.552 & 0.0534 & 0.19500 & -0.16400 \\
\hline-5166.686 & 42.125 & 47.193 & 33.457 & 0.0530 & 0.20200 & -0.17700 \\
\hline-5162.674 & 42.375 & 47.557 & 33.373 & 0.0525 & 0.20700 & -0.18800 \\
\hline-5158.663 & 42.625 & 47.920 & 33.283 & 0.0521 & 0.21400 & -0.19700 \\
\hline-5154.651 & 42.875 & 48.269 & 33.245 & 0.0516 & 0.22100 & -0.20700 \\
\hline-5150.640 & 43.125 & 48.637 & 33.213 & 0.0512 & 0.22600 & -0.21900 \\
\hline-5146.628 & 43.375 & 48.981 & 33.151 & 0.0507 & 0.23400 & -0.23300 \\
\hline-5142.616 & 43.625 & 49.330 & 33.040 & 0.0503 & 0.24300 & -0.24600 \\
\hline-5138.605 & 43.875 & 49.633 & 32.993 & 0.0498 & 0.25000 & -0.26100 \\
\hline-5134.593 & 43.875 & 49.936 & 32.880 & 0.0494 & 0.25600 & -0.27400 \\
\hline-5130.581 & 44.125 & 50.214 & 32.794 & 0.0490 & 0.26100 & -0.29500 \\
\hline-5126.570 & 44.375 & 50.515 & 32.601 & 0.0485 & 0.26600 & -0.31200 \\
\hline-5122.558 & 44.625 & 50.759 & 32.450 & 0.0481 & 0.27100 & -0.33200 \\
\hline-5118.547 & 44.875 & 51.032 & 32.186 & 0.0477 & 0.27500 & -0.35000 \\
\hline-5114.535 & 44.875 & 51.287 & 31.944 & 0.0472 & 0.27700 & -0.37100 \\
\hline-5110.523 & 45.125 & 51.574 & 31.599 & 0.0468 & 0.28400 & -0.39000 \\
\hline-5106.512 & 45.375 & 51.845 & 31.324 & 0.0464 & 0.29100 & -0.41400 \\
\hline-5102.500 & 45.625 & 52.119 & 30.946 & 0.0460 & 0.29500 & -0.44000 \\
\hline-5098.488 & 45.625 & 52.409 & 30.635 & 0.0456 & 0.30000 & -0.46200 \\
\hline-5094.477 & 45.875 & 52.664 & 30.380 & 0.0452 & 0.30300 & -0.48900 \\
\hline-5090.465 & 46.125 & 52.939 & 30.148 & 0.0447 & 0.30700 & -0.51900 \\
\hline-5086.453 & 46.125 & 53.226 & 29.889 & 0.0443 & 0.30900 & -0.54000 \\
\hline-5082.442 & 46.375 & 53.452 & 29.649 & 0.0439 & 0.31600 & -0.55100 \\
\hline-5078.430 & 46.625 & 53.693 & 29.394 & 0.0435 & 0.31700 & -0.57200 \\
\hline-5074.419 & 46.625 & 53.935 & 29.107 & 0.0431 & 0.31700 & -0.59100 \\
\hline-5070.407 & 46.875 & 54.174 & 28.839 & 0.0427 & 0.32100 & -0.59900 \\
\hline-5066.395 & 47.125 & 54.443 & 28.665 & 0.0423 & 0.32600 & -0.60400 \\
\hline-5062.384 & 47.125 & 54.712 & 28.479 & 0.0419 & 0.33100 & -0.60500 \\
\hline-5058.372 & 47.375 & 54.947 & 28.296 & 0.0416 & 0.34000 & -0.59800 \\
\hline-5054.360 & 47.375 & 55.215 & 28.107 & 0.0412 & 0.35000 & -0.59200 \\
\hline-5050.349 & 47.625 & 55.504 & 27.950 & 0.0408 & 0.36200 & -0.58400 \\
\hline-5046.337 & 47.875 & 55.801 & 27.831 & 0.0404 & 0.37200 & -0.57100 \\
\hline-5042.326 & 47.875 & 56.038 & 27.729 & 0.0400 & 0.38600 & -0.56400 \\
\hline-5038.314 & 48.125 & 56.310 & 27.694 & 0.0396 & 0.39600 & -0.54500 \\
\hline-5034.302 & 48.375 & 56.604 & 27.612 & 0.0393 & 0.40800 & -0.52900 \\
\hline-5030.291 & 48.375 & 56.896 & 27.620 & 0.0389 & 0.42000 & -0.50400 \\
\hline-5026.279 & 48.625 & 57.239 & 27.674 & 0.0385 & 0.43500 & -0.47300 \\
\hline-5022.267 & 48.875 & 57.572 & 27.697 & 0.0382 & 0.44900 & -0.44800 \\
\hline-5018.256 & 48.875 & 57.953 & 27.773 & 0.0378 & 0.46900 & -0.42200 \\
\hline-5014.244 & 49.125 & 58.292 & 27.806 & 0.0374 & 0.48300 & -0.40600 \\
\hline-5010.233 & 49.125 & 58.652 & 27.826 & 0.0371 & 0.50400 & -0.39600 \\
\hline-5006.221 & 49.375 & 59.018 & 27.784 & 0.0367 & 0.51700 & -0.38900 \\
\hline-5002.209 & 49.625 & 59.363 & 27.685 & 0.0364 & 0.52500 & -0.39200 \\
\hline-4998.198 & 49.625 & 59.643 & 27.487 & 0.0360 & 0.53700 & -0.40100 \\
\hline-4994.186 & 49.875 & 59.992 & 27.321 & 0.0357 & 0.54300 & -0.41500 \\
\hline-4990.174 & 49.875 & 60.276 & 27.291 & 0.0353 & 0.55100 & -0.43000 \\
\hline-4986.163 & 50.125 & 60.598 & 27.284 & 0.0350 & 0.55700 & -0.45100 \\
\hline-4982.151 & 50.375 & 60.932 & 27.291 & 0.0346 & 0.56300 & -0.47700 \\
\hline-4978.140 & 50.375 & 61.203 & 27.328 & 0.0343 & 0.56400 & -0.50800 \\
\hline-4974.128 & 50.625 & 61.484 & 27.333 & 0.0339 & 0.57000 & -0.53600 \\
\hline-4970.116 & 50.625 & 61.789 & 27.385 & 0.0336 & 0.57900 & -0.57400 \\
\hline
\end{tabular}




$\begin{array}{lllllll}-4966.105 & 50.875 & 62.112 & 27.393 & 0.0333 & 0.59200 & -0.61900 \\ -4962.093 & 50.875 & 62.434 & 27.364 & 0.0329 & 0.60900 & -0.66500 \\ -4958.081 & 51.125 & 62.789 & 27.335 & 0.0326 & 0.62800 & -0.72200 \\ -4954.070 & 51.125 & 63.199 & 27.207 & 0.0323 & 0.66400 & -0.79400 \\ -4950.058 & 51.375 & 63.639 & 27.055 & 0.0320 & 0.70900 & -0.89000 \\ -4946.047 & 51.375 & 64.126 & 26.793 & 0.0316 & 0.77400 & -1.0050 \\ -4942.035 & 51.625 & 64.685 & 26.331 & 0.0313 & 0.87300 & -1.1700 \\ -4938.023 & 51.625 & 65.397 & 25.664 & 0.0310 & 1.0480 & -1.4390 \\ -4934.012 & 51.875 & 66.344 & 24.641 & 0.0307 & 1.3320 & -1.9150 \\ -4930.000 & 52.125 & 68.101 & 22.284 & 0.0304 & 2.0640 & -3.2540\end{array}$




\begin{tabular}{|c|c|c|c|c|c|c|}
\hline Date & Median (muT) & Cred_Int Upper & Cred_Int Lower & dFdt (muT/yr) & dFdt Upper & dFdt Lower \\
\hline-6028.000 & 44.875 & 68.744 & 21.855 & 0.1612 & 3.3890 & -2.7630 \\
\hline-6023.986 & 45.375 & 67.350 & 24.284 & 0.1493 & 2.0180 & -1.6810 \\
\hline-6019.972 & 45.625 & 66.502 & 25.609 & 0.1376 & 1.5360 & -1.2590 \\
\hline-6015.957 & 46.125 & 65.844 & 26.653 & 0.1265 & 1.2680 & -1.0300 \\
\hline-6011.943 & 46.625 & 65.289 & 27.515 & 0.1159 & 1.0930 & -0.87300 \\
\hline-6007.929 & 46.875 & 64.836 & 28.313 & 0.1059 & 0.97100 & -0.76400 \\
\hline-6003.915 & 47.375 & 64.416 & 29.013 & 0.09650 & 0.87900 & -0.68100 \\
\hline-5999.900 & 47.875 & 63.983 & 29.689 & 0.08770 & 0.82400 & -0.62200 \\
\hline-5995.886 & 48.125 & 63.622 & 30.375 & 0.07940 & 0.76900 & -0.58000 \\
\hline-5991.872 & 48.625 & 63.244 & 31.155 & 0.07170 & 0.72800 & -0.55600 \\
\hline-5987.858 & 48.875 & 62.950 & 31.950 & 0.06460 & 0.69800 & -0.53600 \\
\hline-5983.843 & 49.125 & 62.677 & 32.705 & 0.05800 & 0.66800 & -0.52900 \\
\hline-5979.829 & 49.375 & 62.395 & 33.420 & 0.05200 & 0.64400 & -0.53000 \\
\hline-5975.815 & 49.375 & 62.211 & 34.162 & 0.04650 & 0.62400 & -0.52700 \\
\hline-5971.801 & 49.625 & 62.032 & 34.924 & 0.04170 & 0.60200 & -0.52800 \\
\hline-5967.786 & 49.625 & 61.881 & 35.604 & 0.03740 & 0.58600 & -0.53300 \\
\hline-5963.772 & 49.875 & 61.784 & 36.177 & 0.03360 & 0.57000 & -0.54000 \\
\hline-5959.758 & 50.125 & 61.704 & 36.566 & 0.03050 & 0.55500 & -0.54600 \\
\hline-5955.744 & 50.125 & 61.674 & 36.743 & 0.02790 & 0.54700 & -0.55000 \\
\hline-5951.730 & 50.375 & 61.581 & 36.758 & 0.02580 & 0.53200 & -0.56500 \\
\hline-5947.715 & 50.375 & 61.515 & 36.617 & 0.02440 & 0.52600 & -0.57400 \\
\hline-5943.701 & 50.625 & 61.459 & 36.309 & 0.02350 & 0.52000 & -0.58000 \\
\hline-5939.687 & 50.625 & 61.487 & 35.947 & 0.02310 & 0.51100 & -0.58400 \\
\hline-5935.673 & 50.875 & 61.477 & 35.516 & 0.02340 & 0.50300 & -0.59800 \\
\hline-5931.658 & 50.875 & 61.531 & 34.985 & 0.02420 & 0.49500 & -0.60700 \\
\hline-5927.644 & 51.125 & 61.607 & 34.411 & 0.02560 & 0.48700 & -0.61900 \\
\hline-5923.630 & 51.375 & 61.759 & 33.855 & 0.02750 & 0.48500 & -0.62800 \\
\hline-5919.616 & 51.375 & 61.937 & 33.285 & 0.03000 & 0.48400 & -0.64300 \\
\hline-5915.601 & 51.625 & 62.163 & 32.706 & 0.03310 & 0.48400 & -0.65600 \\
\hline-5911.587 & 51.625 & 62.460 & 32.226 & 0.03670 & 0.48900 & -0.67100 \\
\hline-5907.573 & 51.875 & 62.774 & 31.797 & 0.04090 & 0.49700 & -0.68400 \\
\hline-5903.559 & 51.875 & 63.188 & 31.452 & 0.04570 & 0.50900 & -0.69100 \\
\hline-5899.544 & 52.125 & 63.618 & 31.150 & 0.05110 & 0.52500 & -0.70500 \\
\hline-5895.530 & 52.125 & 64.119 & 30.851 & 0.05700 & 0.54300 & -0.69800 \\
\hline-5891.516 & 52.375 & 64.644 & 30.605 & 0.06350 & 0.57400 & -0.69400 \\
\hline-5887.502 & 52.375 & 65.168 & 30.493 & 0.07050 & 0.60300 & -0.68800 \\
\hline-5883.488 & 52.625 & 65.673 & 30.445 & 0.07820 & 0.64100 & -0.68100 \\
\hline-5879.473 & 52.625 & 66.204 & 30.447 & 0.08660 & 0.68200 & -0.66100 \\
\hline-5875.459 & 53.125 & 66.710 & 30.394 & 0.09600 & 0.72600 & -0.64800 \\
\hline-5871.445 & 53.625 & 67.158 & 30.442 & 0.1064 & 0.77500 & -0.63300 \\
\hline-5867.431 & 54.125 & 67.442 & 30.540 & 0.1178 & 0.83700 & -0.61000 \\
\hline-5863.416 & 54.625 & 67.634 & 30.594 & 0.1303 & 0.88700 & -0.59200 \\
\hline-5859.402 & 55.375 & 67.741 & 30.621 & 0.1437 & 0.94200 & -0.56700 \\
\hline-5855.388 & 56.125 & 67.807 & 30.825 & 0.1581 & 1.0040 & -0.53900 \\
\hline-5851.374 & 56.875 & 67.827 & 31.045 & 0.1735 & 1.0740 & -0.50700 \\
\hline-5847.359 & 57.625 & 67.833 & 31.343 & 0.1889 & 1.1320 & -0.47000 \\
\hline-5843.345 & 58.625 & 67.829 & 31.762 & 0.1993 & 1.1820 & -0.44200 \\
\hline-5839.331 & 59.375 & 67.830 & 32.230 & 0.1994 & 1.2330 & -0.41000 \\
\hline-5835.317 & 60.125 & 67.778 & 32.853 & 0.1883 & 1.2810 & -0.37000 \\
\hline-5831.302 & 60.875 & 67.716 & 33.560 & 0.1661 & 1.3080 & -0.34400 \\
\hline-5827.288 & 61.625 & 67.648 & 34.392 & 0.1339 & 1.3200 & -0.31700 \\
\hline
\end{tabular}




\begin{tabular}{|c|c|c|c|c|c|c|}
\hline-5823.274 & 62.125 & 67.565 & 35.371 & 0.09790 & 1.3260 & -0.29400 \\
\hline-5819.260 & 62.375 & 67.484 & 36.505 & 0.06430 & 1.3080 & -0.27300 \\
\hline-5815.246 & 62.375 & 67.410 & 37.864 & 0.03430 & 1.2660 & -0.26700 \\
\hline-5811.231 & 62.375 & 67.306 & 39.163 & 0.007700 & 1.2020 & -0.26800 \\
\hline-5807.217 & 62.125 & 67.184 & 40.487 & -0.01620 & 1.1260 & -0.27300 \\
\hline-5803.203 & 62.125 & 67.077 & 41.905 & -0.03850 & 1.0390 & -0.28000 \\
\hline-5799.189 & 61.875 & 66.936 & 43.597 & -0.05920 & 0.95300 & -0.28800 \\
\hline-5795.174 & 61.625 & 66.814 & 45.116 & -0.07830 & 0.83600 & -0.30200 \\
\hline-5791.160 & 61.375 & 66.635 & 46.905 & -0.09590 & 0.74600 & -0.31700 \\
\hline-5787.146 & 60.875 & 66.399 & 49.007 & -0.1119 & 0.67100 & -0.33300 \\
\hline-5783.132 & 60.375 & 66.116 & 50.621 & -0.1264 & 0.59600 & -0.34900 \\
\hline-5779.117 & 59.875 & 65.814 & 52.410 & -0.1394 & 0.52500 & -0.36300 \\
\hline-5775.103 & 59.125 & 65.542 & 53.226 & -0.1507 & 0.46100 & -0.37700 \\
\hline-5771.089 & 58.625 & 65.195 & 53.438 & -0.1606 & 0.39100 & -0.38900 \\
\hline-5767.075 & 57.875 & 64.848 & 53.119 & -0.1689 & 0.32800 & -0.40300 \\
\hline-5763.060 & 57.125 & 64.442 & 52.599 & -0.1756 & 0.26500 & -0.41500 \\
\hline-5759.046 & 56.375 & 64.028 & 51.979 & -0.1808 & 0.21000 & -0.42300 \\
\hline-5755.032 & 55.875 & 63.519 & 51.307 & -0.1844 & 0.15300 & -0.42900 \\
\hline-5751.018 & 55.125 & 62.910 & 50.550 & -0.1865 & 0.088000 & -0.43500 \\
\hline-5747.004 & 54.375 & 62.149 & 49.733 & -0.1871 & 0.035000 & -0.44000 \\
\hline-5742.989 & 53.625 & 61.237 & 48.830 & -0.1862 & -0.043000 & -0.44800 \\
\hline-5738.975 & 52.875 & 60.188 & 47.918 & -0.1844 & -0.059000 & -0.44700 \\
\hline-5734.961 & 52.375 & 59.053 & 46.970 & -0.1822 & -0.062000 & -0.44700 \\
\hline-5730.947 & 51.625 & 57.940 & 46.014 & -0.1798 & -0.053000 & -0.44300 \\
\hline-5726.932 & 50.875 & 56.822 & 45.110 & -0.1772 & -0.030000 & -0.43600 \\
\hline-5722.918 & 50.125 & 55.774 & 44.281 & -0.1743 & 0.014000 & -0.42700 \\
\hline-5718.904 & 49.625 & 54.708 & 43.510 & -0.1712 & 0.041000 & -0.41800 \\
\hline-5714.890 & 48.875 & 53.673 & 42.786 & -0.1678 & 0.059000 & -0.40800 \\
\hline-5710.875 & 48.125 & 52.727 & 42.056 & -0.1642 & 0.074000 & -0.40000 \\
\hline-5706.861 & 47.375 & 51.880 & 41.310 & -0.1603 & 0.088000 & -0.39000 \\
\hline-5702.847 & 46.875 & 51.122 & 40.523 & -0.1562 & 0.10100 & -0.38000 \\
\hline-5698.833 & 46.125 & 50.445 & 39.765 & -0.1518 & 0.11600 & -0.37400 \\
\hline-5694.819 & 45.625 & 49.841 & 39.028 & -0.1472 & 0.13200 & -0.36900 \\
\hline-5690.804 & 44.875 & 49.352 & 38.299 & -0.1424 & 0.14800 & -0.36500 \\
\hline-5686.790 & 44.375 & 48.996 & 37.622 & -0.1373 & 0.16900 & -0.36000 \\
\hline-5682.776 & 43.625 & 48.797 & 36.928 & -0.1320 & 0.19000 & -0.35500 \\
\hline-5678.762 & 43.125 & 48.761 & 36.300 & -0.1264 & 0.21300 & -0.34900 \\
\hline-5674.747 & 42.625 & 48.816 & 35.637 & -0.1206 & 0.23600 & -0.34600 \\
\hline-5670.733 & 42.125 & 48.903 & 35.072 & -0.1142 & 0.26600 & -0.34400 \\
\hline-5666.719 & 41.875 & 49.053 & 34.570 & -0.1056 & 0.30300 & -0.34300 \\
\hline-5662.705 & 41.375 & 49.239 & 34.015 & -0.09310 & 0.34200 & -0.34000 \\
\hline-5658.690 & 41.125 & 49.482 & 33.515 & -0.07650 & 0.38600 & -0.33800 \\
\hline-5654.676 & 40.875 & 49.769 & 33.059 & -0.05560 & 0.43400 & -0.33700 \\
\hline-5650.662 & 40.625 & 50.206 & 32.625 & -0.03060 & 0.48600 & -0.33400 \\
\hline-5646.648 & 40.625 & 50.674 & 32.244 & -0.001400 & 0.53700 & -0.33300 \\
\hline-5642.633 & 40.625 & 51.137 & 31.930 & 0.03200 & 0.59600 & -0.33200 \\
\hline-5638.619 & 40.875 & 51.702 & 31.624 & 0.06960 & 0.65600 & -0.32700 \\
\hline-5634.605 & 41.125 & 52.405 & 31.402 & 0.1104 & 0.71000 & -0.32100 \\
\hline-5630.591 & 41.875 & 53.299 & 31.256 & 0.1498 & 0.76300 & -0.31700 \\
\hline-5626.577 & 42.375 & 54.262 & 31.217 & 0.1830 & 0.82100 & -0.30900 \\
\hline-5622.562 & 43.125 & 55.294 & 31.184 & 0.2093 & 0.86800 & -0.30100 \\
\hline-5618.548 & 44.125 & 56.343 & 31.243 & 0.2284 & 0.92000 & -0.29100 \\
\hline-5614.534 & 45.125 & 57.216 & 31.369 & 0.2405 & 0.97600 & -0.27300 \\
\hline-5610.520 & 46.125 & 57.884 & 31.655 & 0.2456 & 1.0170 & -0.25800 \\
\hline
\end{tabular}




\begin{tabular}{|c|c|c|c|c|c|c|}
\hline-5606.505 & 47.125 & 58.361 & 31.982 & 0.2435 & 1.0640 & -0.24700 \\
\hline-5602.491 & 48.125 & 58.786 & 32.411 & 0.2348 & 1.0690 & -0.24500 \\
\hline-5598.477 & 48.875 & 59.028 & 33.079 & 0.2207 & 1.0720 & -0.24700 \\
\hline-5594.463 & 49.875 & 59.244 & 33.902 & 0.2027 & 1.0480 & -0.25800 \\
\hline-5590.448 & 50.625 & 59.471 & 35.087 & 0.1812 & 1.0120 & -0.27700 \\
\hline-5586.434 & 51.375 & 59.681 & 36.515 & 0.1562 & 0.96400 & -0.29800 \\
\hline-5582.420 & 51.625 & 59.894 & 38.331 & 0.1275 & 0.92100 & -0.32000 \\
\hline-5578.406 & 52.125 & 60.153 & 40.317 & 0.09530 & 0.87300 & -0.34800 \\
\hline-5574.391 & 52.375 & 60.365 & 42.262 & 0.05950 & 0.81300 & -0.38000 \\
\hline-5570.377 & 52.375 & 60.529 & 43.905 & 0.02020 & 0.75700 & -0.41200 \\
\hline-5566.363 & 52.375 & 60.637 & 45.071 & -0.02200 & 0.69600 & -0.44900 \\
\hline-5562.349 & 52.375 & 60.669 & 45.469 & -0.06330 & 0.63500 & -0.48400 \\
\hline-5558.335 & 52.375 & 60.609 & 45.095 & -0.1002 & 0.56400 & -0.52200 \\
\hline-5554.320 & 52.125 & 60.494 & 44.399 & -0.1318 & 0.49100 & -0.56900 \\
\hline-5550.306 & 51.625 & 60.328 & 43.587 & -0.1582 & 0.43000 & -0.60500 \\
\hline-5546.292 & 50.875 & 59.977 & 42.788 & -0.1793 & 0.36800 & -0.65100 \\
\hline-5542.278 & 49.875 & 59.550 & 42.030 & -0.1953 & 0.30900 & -0.68100 \\
\hline-5538.263 & 49.125 & 58.954 & 41.358 & -0.2060 & 0.26200 & -0.70200 \\
\hline-5534.249 & 48.125 & 58.228 & 40.723 & -0.2115 & 0.21900 & -0.70300 \\
\hline-5530.235 & 47.125 & 57.458 & 40.072 & -0.2122 & 0.17800 & -0.69200 \\
\hline-5526.221 & 46.125 & 56.572 & 39.383 & -0.2107 & 0.13700 & -0.67000 \\
\hline-5522.206 & 45.375 & 55.533 & 38.732 & -0.2095 & 0.10100 & -0.64200 \\
\hline-5518.192 & 44.375 & 54.341 & 38.064 & -0.2091 & 0.055000 & -0.61600 \\
\hline-5514.178 & 43.625 & 53.014 & 37.350 & -0.2094 & -0.022000 & -0.59300 \\
\hline-5510.164 & 42.625 & 51.498 & 36.518 & -0.2105 & -0.059000 & -0.56800 \\
\hline-5506.149 & 41.875 & 50.018 & 35.678 & -0.2123 & -0.064000 & -0.54500 \\
\hline-5502.135 & 41.125 & 48.628 & 34.872 & -0.2149 & -0.065000 & -0.52300 \\
\hline-5498.121 & 40.125 & 47.260 & 34.030 & -0.2182 & -0.062000 & -0.50400 \\
\hline-5494.107 & 39.375 & 45.981 & 33.085 & -0.2220 & -0.057000 & -0.48700 \\
\hline-5490.093 & 38.625 & 44.747 & 31.990 & -0.2245 & -0.049000 & -0.47200 \\
\hline-5486.078 & 37.875 & 43.583 & 30.843 & -0.2240 & -0.015000 & -0.45900 \\
\hline-5482.064 & 37.125 & 42.476 & 29.931 & -0.2201 & 0.049000 & -0.44600 \\
\hline-5478.050 & 36.375 & 41.411 & 29.455 & -0.2129 & 0.053000 & -0.43300 \\
\hline-5474.036 & 35.625 & 40.368 & 29.036 & -0.2024 & 0.057000 & -0.42100 \\
\hline-5470.021 & 34.875 & 39.385 & 28.667 & -0.1885 & 0.060000 & -0.40700 \\
\hline-5466.007 & 34.125 & 38.446 & 28.344 & -0.1713 & 0.063000 & -0.39500 \\
\hline-5461.993 & 33.375 & 37.558 & 28.069 & -0.1511 & 0.066000 & -0.38000 \\
\hline-5457.979 & 32.625 & 36.740 & 27.834 & -0.1298 & 0.070000 & -0.36400 \\
\hline-5453.964 & 31.875 & 35.965 & 27.663 & -0.1092 & 0.075000 & -0.34700 \\
\hline-5449.950 & 31.375 & 35.276 & 27.537 & -0.08990 & 0.082000 & -0.33100 \\
\hline-5445.936 & 30.875 & 34.654 & 27.440 & -0.07160 & 0.090000 & -0.31300 \\
\hline-5441.922 & 30.625 & 34.081 & 27.364 & -0.05460 & 0.099000 & -0.28900 \\
\hline-5437.907 & 30.375 & 33.561 & 27.295 & -0.03860 & 0.10900 & -0.26700 \\
\hline-5433.893 & 30.375 & 33.106 & 27.251 & -0.02380 & 0.11900 & -0.24500 \\
\hline-5429.879 & 30.375 & 32.828 & 27.221 & -0.01020 & 0.12900 & -0.22500 \\
\hline-5425.865 & 30.375 & 32.775 & 27.202 & 0.002300 & 0.13800 & -0.20600 \\
\hline-5421.851 & 30.375 & 32.881 & 27.220 & 0.01360 & 0.15000 & -0.18800 \\
\hline-5417.836 & 30.375 & 33.085 & 27.246 & 0.02380 & 0.16200 & -0.17200 \\
\hline-5413.822 & 30.625 & 33.351 & 27.295 & 0.03280 & 0.17300 & -0.15600 \\
\hline-5409.808 & 30.875 & 33.638 & 27.348 & 0.04070 & 0.18400 & -0.14200 \\
\hline-5405.794 & 31.125 & 33.950 & 27.423 & 0.04750 & 0.19400 & -0.12900 \\
\hline-5401.779 & 31.375 & 34.324 & 27.509 & 0.05300 & 0.20400 & -0.11500 \\
\hline-5397.765 & 31.625 & 34.724 & 27.645 & 0.05750 & 0.21400 & -0.10200 \\
\hline-5393.751 & 31.875 & 35.151 & 27.791 & 0.06080 & 0.22400 & -0.091000 \\
\hline
\end{tabular}




\begin{tabular}{|c|c|c|c|c|c|c|}
\hline-5389.737 & 32.125 & 35.609 & 27.983 & 0.06300 & 0.23300 & -0.082000 \\
\hline-5385.722 & 32.375 & 36.101 & 28.184 & 0.06460 & 0.24100 & -0.073000 \\
\hline-5381.708 & 32.625 & 36.554 & 28.378 & 0.06590 & 0.24300 & -0.066000 \\
\hline-5377.694 & 32.875 & 37.024 & 28.592 & 0.06710 & 0.24600 & -0.056000 \\
\hline-5373.680 & 33.125 & 37.486 & 28.885 & 0.06810 & 0.24700 & -0.047000 \\
\hline-5369.665 & 33.625 & 37.901 & 29.210 & 0.06900 & 0.24300 & -0.035000 \\
\hline-5365.651 & 33.875 & 38.302 & 29.628 & 0.06970 & 0.23800 & -0.025000 \\
\hline-5361.637 & 34.125 & 38.661 & 30.142 & 0.07030 & 0.23400 & -0.016000 \\
\hline-5357.623 & 34.375 & 39.016 & 30.750 & 0.07070 & 0.22200 & -0.012000 \\
\hline-5353.609 & 34.625 & 39.315 & 31.332 & 0.07090 & 0.21200 & -0.0100000 \\
\hline-5349.594 & 34.875 & 39.544 & 31.885 & 0.07100 & 0.19900 & -0.0090000 \\
\hline-5345.580 & 35.125 & 39.771 & 32.383 & 0.07090 & 0.18700 & -0.0090000 \\
\hline-5341.566 & 35.375 & 39.917 & 32.840 & 0.07070 & 0.17300 & -0.011000 \\
\hline-5337.552 & 35.625 & 40.018 & 33.238 & 0.07030 & 0.16200 & -0.011000 \\
\hline-5333.537 & 35.875 & 40.076 & 33.612 & 0.06980 & 0.15000 & -0.013000 \\
\hline-5329.523 & 36.125 & 40.103 & 33.965 & 0.06910 & 0.13900 & -0.014000 \\
\hline-5325.509 & 36.375 & 40.124 & 34.296 & 0.06820 & 0.13000 & -0.015000 \\
\hline-5321.495 & 36.625 & 40.162 & 34.617 & 0.06720 & 0.12300 & -0.016000 \\
\hline-5317.480 & 36.875 & 40.216 & 34.919 & 0.06610 & 0.11600 & -0.016000 \\
\hline-5313.466 & 37.125 & 40.289 & 35.208 & 0.06510 & 0.11100 & -0.015000 \\
\hline-5309.452 & 37.375 & 40.382 & 35.490 & 0.06420 & 0.10700 & -0.014000 \\
\hline-5305.438 & 37.625 & 40.486 & 35.761 & 0.06320 & 0.10400 & -0.013000 \\
\hline-5301.423 & 37.875 & 40.601 & 36.029 & 0.06230 & 0.10100 & -0.011000 \\
\hline-5297.409 & 38.125 & 40.748 & 36.288 & 0.06150 & 0.099000 & -0.0100000 \\
\hline-5293.395 & 38.375 & 40.902 & 36.540 & 0.06070 & 0.097000 & -0.0090000 \\
\hline-5289.381 & 38.625 & 41.075 & 36.786 & 0.06000 & 0.096000 & -0.0070000 \\
\hline-5285.367 & 38.875 & 41.245 & 37.028 & 0.05930 & 0.095000 & -0.0060000 \\
\hline-5281.352 & 39.125 & 41.432 & 37.263 & 0.05860 & 0.094000 & -0.0040000 \\
\hline-5277.338 & 39.375 & 41.630 & 37.489 & 0.05800 & 0.093000 & -0.0020000 \\
\hline-5273.324 & 39.625 & 41.836 & 37.708 & 0.05750 & 0.092000 & -0.0010000 \\
\hline-5269.310 & 39.875 & 42.057 & 37.919 & 0.05690 & 0.091000 & 0.0020000 \\
\hline-5265.295 & 40.125 & 42.277 & 38.120 & 0.05650 & 0.091000 & 0.0040000 \\
\hline-5261.281 & 40.375 & 42.506 & 38.308 & 0.05610 & 0.091000 & 0.0050000 \\
\hline-5257.267 & 40.625 & 42.741 & 38.490 & 0.05570 & 0.090000 & 0.0070000 \\
\hline-5253.253 & 40.875 & 42.985 & 38.667 & 0.05540 & 0.090000 & 0.0100000 \\
\hline-5249.238 & 41.125 & 43.222 & 38.847 & 0.05510 & 0.091000 & 0.012000 \\
\hline-5245.224 & 41.375 & 43.468 & 39.022 & 0.05480 & 0.091000 & 0.013000 \\
\hline-5241.210 & 41.625 & 43.716 & 39.193 & 0.05460 & 0.091000 & 0.015000 \\
\hline-5237.196 & 41.625 & 43.958 & 39.383 & 0.05450 & 0.091000 & 0.015000 \\
\hline-5233.181 & 41.875 & 44.199 & 39.574 & 0.05440 & 0.092000 & 0.016000 \\
\hline-5229.167 & 42.125 & 44.447 & 39.770 & 0.05440 & 0.092000 & 0.016000 \\
\hline-5225.153 & 42.375 & 44.678 & 39.983 & 0.05440 & 0.092000 & 0.016000 \\
\hline-5221.139 & 42.625 & 44.913 & 40.197 & 0.05440 & 0.093000 & 0.015000 \\
\hline-5217.125 & 42.875 & 45.145 & 40.423 & 0.05450 & 0.094000 & 0.015000 \\
\hline-5213.110 & 43.125 & 45.379 & 40.644 & 0.05460 & 0.094000 & 0.014000 \\
\hline-5209.096 & 43.375 & 45.609 & 40.877 & 0.05480 & 0.095000 & 0.013000 \\
\hline-5205.082 & 43.625 & 45.819 & 41.110 & 0.05500 & 0.095000 & 0.012000 \\
\hline-5201.068 & 43.875 & 46.039 & 41.342 & 0.05530 & 0.096000 & 0.0100000 \\
\hline-5197.053 & 44.125 & 46.263 & 41.564 & 0.05560 & 0.096000 & 0.0080000 \\
\hline-5193.039 & 44.375 & 46.495 & 41.784 & 0.05600 & 0.097000 & 0.0060000 \\
\hline-5189.025 & 44.625 & 46.747 & 41.999 & 0.05640 & 0.097000 & 0.0040000 \\
\hline-5185.011 & 44.875 & 47.004 & 42.207 & 0.05690 & 0.098000 & 0.0020000 \\
\hline-5180.996 & 45.125 & 47.270 & 42.423 & 0.05740 & 0.10000 & -0.0010000 \\
\hline-5176.982 & 45.125 & 47.547 & 42.635 & 0.05790 & 0.10100 & -0.0030000 \\
\hline
\end{tabular}




\begin{tabular}{|c|c|c|c|c|c|c|}
\hline-5172.968 & 45.375 & 47.834 & 42.841 & 0.05830 & 0.10300 & -0.0060000 \\
\hline-5168.954 & 45.625 & 48.128 & 43.035 & 0.05870 & 0.10400 & -0.0100000 \\
\hline-5164.940 & 45.875 & 48.420 & 43.219 & 0.05910 & 0.10600 & -0.013000 \\
\hline-5160.925 & 46.125 & 48.722 & 43.397 & 0.05930 & 0.10900 & -0.016000 \\
\hline-5156.911 & 46.375 & 49.032 & 43.567 & 0.05960 & 0.11200 & -0.019000 \\
\hline-5152.897 & 46.625 & 49.342 & 43.725 & 0.05980 & 0.11500 & -0.022000 \\
\hline-5148.883 & 46.625 & 49.665 & 43.890 & 0.05990 & 0.11800 & -0.025000 \\
\hline-5144.868 & 46.875 & 50.011 & 44.037 & 0.05990 & 0.12200 & -0.028000 \\
\hline-5140.854 & 47.125 & 50.354 & 44.181 & 0.06000 & 0.12700 & -0.031000 \\
\hline-5136.840 & 47.375 & 50.707 & 44.313 & 0.05990 & 0.13300 & -0.034000 \\
\hline-5132.826 & 47.625 & 51.071 & 44.436 & 0.05980 & 0.13900 & -0.038000 \\
\hline-5128.811 & 47.875 & 51.434 & 44.547 & 0.05970 & 0.14300 & -0.044000 \\
\hline-5124.797 & 48.125 & 51.827 & 44.632 & 0.05950 & 0.15000 & -0.049000 \\
\hline-5120.783 & 48.375 & 52.227 & 44.727 & 0.05920 & 0.15700 & -0.053000 \\
\hline-5116.769 & 48.625 & 52.646 & 44.783 & 0.05890 & 0.16400 & -0.060000 \\
\hline-5112.754 & 48.625 & 53.062 & 44.837 & 0.05860 & 0.17200 & -0.066000 \\
\hline-5108.740 & 48.875 & 53.504 & 44.847 & 0.05810 & 0.17900 & -0.073000 \\
\hline-5104.726 & 49.125 & 53.963 & 44.868 & 0.05770 & 0.18700 & -0.080000 \\
\hline-5100.712 & 49.375 & 54.402 & 44.807 & 0.05720 & 0.19200 & -0.091000 \\
\hline-5096.698 & 49.625 & 54.836 & 44.752 & 0.05660 & 0.19900 & -0.10000 \\
\hline-5092.683 & 49.875 & 55.225 & 44.701 & 0.05590 & 0.20500 & -0.11100 \\
\hline-5088.669 & 50.125 & 55.649 & 44.655 & 0.05530 & 0.20800 & -0.12500 \\
\hline-5084.655 & 50.375 & 56.012 & 44.567 & 0.05450 & 0.21200 & -0.13800 \\
\hline-5080.641 & 50.625 & 56.397 & 44.513 & 0.05370 & 0.21400 & -0.15100 \\
\hline-5076.626 & 50.875 & 56.702 & 44.438 & 0.05290 & 0.21800 & -0.16300 \\
\hline-5072.612 & 50.875 & 57.036 & 44.372 & 0.05200 & 0.22200 & -0.17600 \\
\hline-5068.598 & 51.125 & 57.348 & 44.258 & 0.05100 & 0.22400 & -0.19200 \\
\hline-5064.584 & 51.375 & 57.654 & 44.097 & 0.05000 & 0.22600 & -0.20800 \\
\hline-5060.569 & 51.625 & 57.961 & 43.949 & 0.04890 & 0.22900 & -0.22500 \\
\hline-5056.555 & 51.875 & 58.240 & 43.771 & 0.04780 & 0.23200 & -0.24100 \\
\hline-5052.541 & 51.875 & 58.526 & 43.511 & 0.04660 & 0.23600 & -0.25800 \\
\hline-5048.527 & 52.125 & 58.769 & 43.244 & 0.04540 & 0.24100 & -0.27500 \\
\hline-5044.512 & 52.375 & 59.073 & 42.894 & 0.04410 & 0.24600 & -0.28900 \\
\hline-5040.498 & 52.625 & 59.329 & 42.530 & 0.04280 & 0.25100 & -0.30700 \\
\hline-5036.484 & 52.625 & 59.577 & 42.060 & 0.04140 & 0.25600 & -0.32100 \\
\hline-5032.470 & 52.875 & 59.836 & 41.631 & 0.04000 & 0.25900 & -0.33700 \\
\hline-5028.456 & 53.125 & 60.093 & 41.171 & 0.03850 & 0.26500 & -0.34900 \\
\hline-5024.441 & 53.125 & 60.320 & 40.629 & 0.03690 & 0.27100 & -0.36700 \\
\hline-5020.427 & 53.375 & 60.563 & 40.069 & 0.03530 & 0.27700 & -0.38500 \\
\hline-5016.413 & 53.625 & 60.793 & 39.464 & 0.03370 & 0.28300 & -0.40100 \\
\hline-5012.399 & 53.625 & 61.010 & 38.805 & 0.03190 & 0.28700 & -0.42100 \\
\hline-5008.384 & 53.875 & 61.253 & 38.140 & 0.03020 & 0.29200 & -0.43500 \\
\hline-5004.370 & 53.875 & 61.457 & 37.489 & 0.02840 & 0.30100 & -0.45300 \\
\hline-5000.356 & 54.125 & 61.661 & 36.892 & 0.02650 & 0.30600 & -0.46800 \\
\hline-4996.342 & 54.125 & 61.837 & 36.278 & 0.02460 & 0.31200 & -0.48400 \\
\hline-4992.327 & 54.375 & 62.056 & 35.690 & 0.02260 & 0.32200 & -0.49600 \\
\hline-4988.313 & 54.375 & 62.269 & 35.118 & 0.02050 & 0.33300 & -0.51200 \\
\hline-4984.299 & 54.625 & 62.437 & 34.527 & 0.01840 & 0.34200 & -0.52200 \\
\hline-4980.285 & 54.625 & 62.616 & 34.076 & 0.01630 & 0.35100 & -0.53100 \\
\hline-4976.270 & 54.625 & 62.789 & 33.513 & 0.01410 & 0.35800 & -0.54700 \\
\hline-4972.256 & 54.875 & 62.985 & 33.083 & 0.01180 & 0.36600 & -0.56200 \\
\hline-4968.242 & 54.875 & 63.183 & 32.561 & 0.009500 & 0.37800 & -0.57100 \\
\hline-4964.228 & 54.875 & 63.374 & 32.113 & 0.007200 & 0.38900 & -0.59100 \\
\hline-4960.214 & 54.875 & 63.582 & 31.704 & 0.004800 & 0.40100 & -0.61300 \\
\hline
\end{tabular}




$\begin{array}{lllllll}-4956.199 & 54.875 & 63.802 & 31.241 & 0.002300 & 0.41400 & -0.62800 \\ -4952.185 & 54.875 & 64.020 & 30.843 & -0.0002000 & 0.43000 & -0.64800 \\ -4948.171 & 54.875 & 64.233 & 30.491 & -0.002800 & 0.44700 & -0.67100 \\ -4944.157 & 54.875 & 64.443 & 30.142 & -0.005500 & 0.46700 & -0.69500 \\ -4940.142 & 54.875 & 64.657 & 29.776 & -0.008100 & 0.49700 & -0.72400 \\ -4936.128 & 54.625 & 64.894 & 29.488 & -0.01090 & 0.53100 & -0.76000 \\ -4932.114 & 54.625 & 65.128 & 29.116 & -0.01370 & 0.56300 & -0.80700 \\ -4928.100 & 54.625 & 65.375 & 28.719 & -0.01650 & 0.60500 & -0.86600 \\ -4924.085 & 54.375 & 65.615 & 28.295 & -0.01940 & 0.66300 & -0.93300 \\ -4920.071 & 54.375 & 65.854 & 27.894 & -0.02240 & 0.73600 & -1.0290 \\ -4916.057 & 54.125 & 66.139 & 27.339 & -0.02540 & 0.83500 & -1.1620 \\ -4912.043 & 53.875 & 66.471 & 26.674 & -0.02850 & 0.96200 & -1.3440 \\ -4908.028 & 53.625 & 66.850 & 25.767 & -0.03160 & 1.1500 & -1.6510 \\ -4904.014 & 53.625 & 67.364 & 24.489 & -0.03470 & 1.5270 & -2.2240 \\ -4900.000 & 53.625 & 68.491 & 21.969 & -0.03790 & 2.4580 & -3.9120\end{array}$




\begin{tabular}{|c|c|c|c|c|c|c|}
\hline Date & Median (muT) & Cred_Int Upper & Cred_Int Lower & dFdt (muT/yr) & dFdt Upper & dFdt Lower \\
\hline-6028.000 & 44.875 & 68.540 & 21.791 & 0.1048 & 3.4980 & -2.8920 \\
\hline-6023.986 & 45.375 & 66.878 & 23.932 & 0.09610 & 2.0660 & -1.7530 \\
\hline-6019.972 & 45.875 & 65.958 & 25.057 & 0.08770 & 1.5160 & -1.3300 \\
\hline-6015.957 & 46.125 & 65.240 & 25.956 & 0.07970 & 1.2360 & -1.0950 \\
\hline-6011.943 & 46.375 & 64.661 & 26.689 & 0.07240 & 1.0620 & -0.95700 \\
\hline-6007.929 & 46.625 & 64.230 & 27.309 & 0.06560 & 0.94500 & -0.85100 \\
\hline-6003.915 & 46.875 & 63.821 & 27.895 & 0.05930 & 0.85200 & -0.78100 \\
\hline-5999.900 & 47.125 & 63.471 & 28.469 & 0.05360 & 0.78300 & -0.73100 \\
\hline-5995.886 & 47.375 & 63.189 & 29.026 & 0.04840 & 0.73400 & -0.68500 \\
\hline-5991.872 & 47.375 & 62.933 & 29.607 & 0.04380 & 0.68600 & -0.65800 \\
\hline-5987.858 & 47.625 & 62.678 & 30.141 & 0.03970 & 0.65300 & -0.63600 \\
\hline-5983.843 & 47.625 & 62.398 & 30.626 & 0.03620 & 0.63200 & -0.61400 \\
\hline-5979.829 & 47.875 & 62.225 & 31.051 & 0.03320 & 0.61100 & -0.60000 \\
\hline-5975.815 & 47.875 & 62.013 & 31.442 & 0.03080 & 0.59000 & -0.59400 \\
\hline-5971.801 & 48.125 & 61.858 & 31.789 & 0.02890 & 0.57500 & -0.58300 \\
\hline-5967.786 & 48.125 & 61.685 & 32.025 & 0.02760 & 0.55800 & -0.58000 \\
\hline-5963.772 & 48.375 & 61.515 & 32.268 & 0.02680 & 0.54600 & -0.57800 \\
\hline-5959.758 & 48.375 & 61.328 & 32.355 & 0.02660 & 0.54000 & -0.57700 \\
\hline-5955.744 & 48.625 & 61.217 & 32.488 & 0.02690 & 0.53400 & -0.58000 \\
\hline-5951.730 & 48.625 & 61.130 & 32.569 & 0.02770 & 0.52900 & -0.57500 \\
\hline-5947.715 & 48.875 & 61.115 & 32.608 & 0.02910 & 0.52400 & -0.57600 \\
\hline-5943.701 & 49.125 & 61.043 & 32.581 & 0.03110 & 0.52200 & -0.57700 \\
\hline-5939.687 & 49.125 & 61.029 & 32.501 & 0.03360 & 0.52500 & -0.58100 \\
\hline-5935.673 & 49.375 & 61.020 & 32.453 & 0.03660 & 0.52500 & -0.58600 \\
\hline-5931.658 & 49.625 & 61.086 & 32.312 & 0.04020 & 0.52000 & -0.59700 \\
\hline-5927.644 & 49.875 & 61.134 & 32.199 & 0.04430 & 0.52300 & -0.59700 \\
\hline-5923.630 & 49.875 & 61.248 & 32.123 & 0.04900 & 0.53100 & -0.60700 \\
\hline-5919.616 & 50.125 & 61.407 & 31.911 & 0.05430 & 0.53600 & -0.61000 \\
\hline-5915.601 & 50.375 & 61.664 & 31.768 & 0.06000 & 0.54600 & -0.61700 \\
\hline-5911.587 & 50.625 & 61.904 & 31.617 & 0.06640 & 0.55700 & -0.62000 \\
\hline-5907.573 & 50.875 & 62.225 & 31.453 & 0.07320 & 0.56400 & -0.63100 \\
\hline-5903.559 & 51.125 & 62.614 & 31.264 & 0.08070 & 0.57900 & -0.63200 \\
\hline-5899.544 & 51.375 & 63.048 & 31.189 & 0.08860 & 0.59400 & -0.63700 \\
\hline-5895.530 & 51.875 & 63.583 & 31.076 & 0.09720 & 0.61700 & -0.63600 \\
\hline-5891.516 & 52.125 & 64.133 & 30.994 & 0.1062 & 0.64100 & -0.63200 \\
\hline-5887.502 & 52.625 & 64.745 & 30.833 & 0.1158 & 0.67200 & -0.63200 \\
\hline-5883.488 & 52.875 & 65.401 & 30.855 & 0.1258 & 0.70200 & -0.63000 \\
\hline-5879.473 & 53.375 & 66.012 & 30.891 & 0.1354 & 0.73600 & -0.62000 \\
\hline-5875.459 & 53.875 & 66.602 & 31.071 & 0.1438 & 0.77900 & -0.61300 \\
\hline-5871.445 & 54.625 & 67.104 & 31.226 & 0.1508 & 0.82200 & -0.59800 \\
\hline-5867.431 & 55.125 & 67.447 & 31.432 & 0.1564 & 0.88000 & -0.57400 \\
\hline-5863.416 & 55.625 & 67.621 & 31.661 & 0.1607 & 0.94200 & -0.55300 \\
\hline-5859.402 & 56.375 & 67.715 & 31.915 & 0.1636 & 0.99100 & -0.53100 \\
\hline-5855.388 & 57.125 & 67.734 & 32.251 & 0.1651 & 1.0530 & -0.49600 \\
\hline-5851.374 & 57.625 & 67.712 & 32.644 & 0.1652 & 1.1170 & -0.45900 \\
\hline-5847.359 & 58.375 & 67.661 & 33.092 & 0.1637 & 1.1740 & -0.42600 \\
\hline-5843.345 & 59.125 & 67.616 & 33.716 & 0.1592 & 1.2090 & -0.39000 \\
\hline-5839.331 & 59.875 & 67.571 & 34.365 & 0.1505 & 1.2270 & -0.35500 \\
\hline-5835.317 & 60.875 & 67.505 & 34.982 & 0.1371 & 1.2380 & -0.32300 \\
\hline-5831.302 & 61.625 & 67.414 & 35.896 & 0.1192 & 1.2220 & -0.30200 \\
\hline-5827.288 & 62.125 & 67.339 & 36.950 & 0.09700 & 1.1620 & -0.28400 \\
\hline
\end{tabular}




\begin{tabular}{|c|c|c|c|c|c|c|}
\hline-5823.274 & 62.375 & 67.221 & 37.985 & 0.07210 & 1.1100 & -0.28500 \\
\hline-5819.260 & 62.375 & 67.114 & 39.103 & 0.04590 & 1.0500 & -0.29200 \\
\hline-5815.246 & 62.375 & 67.006 & 40.286 & 0.01880 & 0.98100 & -0.30200 \\
\hline-5811.231 & 62.125 & 66.927 & 41.491 & -0.008900 & 0.90800 & -0.31400 \\
\hline-5807.217 & 62.125 & 66.833 & 42.761 & -0.03560 & 0.83800 & -0.32900 \\
\hline-5803.203 & 61.875 & 66.721 & 43.768 & -0.05980 & 0.78300 & -0.34300 \\
\hline-5799.189 & 61.625 & 66.623 & 44.780 & -0.08100 & 0.72800 & -0.36500 \\
\hline-5795.174 & 61.125 & 66.470 & 45.687 & -0.09930 & 0.67900 & -0.37900 \\
\hline-5791.160 & 60.625 & 66.297 & 46.564 & -0.1147 & 0.62200 & -0.39200 \\
\hline-5787.146 & 60.125 & 66.121 & 47.517 & -0.1271 & 0.58100 & -0.40000 \\
\hline-5783.132 & 59.625 & 65.931 & 48.337 & -0.1367 & 0.54000 & -0.40700 \\
\hline-5779.117 & 59.125 & 65.712 & 48.997 & -0.1433 & 0.49200 & -0.41600 \\
\hline-5775.103 & 58.625 & 65.482 & 49.487 & -0.1473 & 0.44700 & -0.42700 \\
\hline-5771.089 & 58.125 & 65.198 & 49.475 & -0.1497 & 0.40500 & -0.43200 \\
\hline-5767.075 & 57.375 & 64.927 & 49.323 & -0.1517 & 0.36500 & -0.44100 \\
\hline-5763.060 & 56.875 & 64.612 & 48.997 & -0.1536 & 0.32800 & -0.44700 \\
\hline-5759.046 & 56.125 & 64.335 & 48.574 & -0.1553 & 0.28500 & -0.45600 \\
\hline-5755.032 & 55.625 & 63.941 & 48.021 & -0.1569 & 0.24100 & -0.46300 \\
\hline-5751.018 & 54.875 & 63.459 & 47.490 & -0.1583 & 0.20600 & -0.46800 \\
\hline-5747.004 & 54.375 & 62.918 & 47.053 & -0.1596 & 0.17800 & -0.46900 \\
\hline-5742.989 & 53.625 & 62.262 & 46.488 & -0.1606 & 0.15600 & -0.46800 \\
\hline-5738.975 & 52.875 & 61.511 & 45.778 & -0.1613 & 0.13800 & -0.46300 \\
\hline-5734.961 & 52.375 & 60.727 & 45.111 & -0.1616 & 0.12300 & -0.45800 \\
\hline-5730.947 & 51.625 & 59.908 & 44.444 & -0.1613 & 0.11600 & -0.45100 \\
\hline-5726.932 & 51.125 & 58.986 & 43.755 & -0.1606 & 0.11100 & -0.44500 \\
\hline-5722.918 & 50.375 & 58.065 & 43.157 & -0.1593 & 0.11300 & -0.43500 \\
\hline-5718.904 & 49.875 & 57.176 & 42.589 & -0.1575 & 0.12000 & -0.42600 \\
\hline-5714.890 & 49.125 & 56.361 & 42.007 & -0.1553 & 0.12700 & -0.41600 \\
\hline-5710.875 & 48.625 & 55.544 & 41.386 & -0.1525 & 0.13400 & -0.40700 \\
\hline-5706.861 & 48.125 & 54.740 & 40.821 & -0.1492 & 0.14300 & -0.39700 \\
\hline-5702.847 & 47.375 & 53.901 & 40.258 & -0.1454 & 0.15200 & -0.38900 \\
\hline-5698.833 & 46.875 & 53.085 & 39.720 & -0.1411 & 0.16400 & -0.38100 \\
\hline-5694.819 & 46.375 & 52.333 & 39.163 & -0.1363 & 0.17600 & -0.37500 \\
\hline-5690.804 & 45.875 & 51.648 & 38.625 & -0.1310 & 0.18900 & -0.37100 \\
\hline-5686.790 & 45.375 & 51.024 & 38.082 & -0.1252 & 0.20200 & -0.36600 \\
\hline-5682.776 & 44.875 & 50.511 & 37.537 & -0.1189 & 0.21700 & -0.36400 \\
\hline-5678.762 & 44.375 & 50.068 & 37.019 & -0.1121 & 0.23300 & -0.36300 \\
\hline-5674.747 & 43.875 & 49.828 & 36.521 & -0.1047 & 0.25400 & -0.36400 \\
\hline-5670.733 & 43.625 & 49.730 & 36.051 & -0.09670 & 0.27800 & -0.36800 \\
\hline-5666.719 & 43.125 & 49.801 & 35.559 & -0.08670 & 0.30700 & -0.36800 \\
\hline-5662.705 & 42.875 & 49.995 & 35.094 & -0.07370 & 0.34000 & -0.36900 \\
\hline-5658.690 & 42.625 & 50.274 & 34.645 & -0.05750 & 0.38200 & -0.36500 \\
\hline-5654.676 & 42.125 & 50.638 & 34.220 & -0.03800 & 0.42200 & -0.36000 \\
\hline-5650.662 & 42.125 & 51.079 & 33.799 & -0.01530 & 0.46600 & -0.35700 \\
\hline-5646.648 & 41.875 & 51.558 & 33.449 & 0.01080 & 0.52000 & -0.35600 \\
\hline-5642.633 & 41.875 & 52.042 & 33.159 & 0.04000 & 0.57400 & -0.35600 \\
\hline-5638.619 & 42.125 & 52.651 & 32.913 & 0.07260 & 0.63400 & -0.35100 \\
\hline-5634.605 & 42.375 & 53.292 & 32.733 & 0.1075 & 0.71000 & -0.33400 \\
\hline-5630.591 & 42.625 & 54.125 & 32.623 & 0.1408 & 0.78100 & -0.32100 \\
\hline-5626.577 & 43.375 & 55.016 & 32.641 & 0.1681 & 0.85100 & -0.31700 \\
\hline-5622.562 & 44.375 & 55.744 & 32.695 & 0.1886 & 0.92200 & -0.31000 \\
\hline-5618.548 & 45.125 & 56.463 & 32.778 & 0.2024 & 0.98900 & -0.30400 \\
\hline-5614.534 & 46.125 & 57.158 & 32.945 & 0.2094 & 1.0270 & -0.29900 \\
\hline-5610.520 & 47.125 & 57.743 & 33.207 & 0.2097 & 1.0600 & -0.29600 \\
\hline
\end{tabular}




\begin{tabular}{|c|c|c|c|c|c|c|}
\hline-5606.505 & 48.125 & 58.274 & 33.459 & 0.2032 & 1.0830 & -0.30000 \\
\hline-5602.491 & 49.125 & 58.635 & 33.776 & 0.1904 & 1.0910 & -0.30500 \\
\hline-5598.477 & 50.125 & 58.955 & 34.182 & 0.1731 & 1.0710 & -0.31800 \\
\hline-5594.463 & 50.625 & 59.233 & 34.714 & 0.1531 & 1.0360 & -0.35100 \\
\hline-5590.448 & 51.125 & 59.487 & 35.340 & 0.1310 & 0.99000 & -0.38700 \\
\hline-5586.434 & 51.625 & 59.726 & 36.243 & 0.1066 & 0.95100 & -0.42600 \\
\hline-5582.420 & 51.875 & 59.902 & 37.280 & 0.08000 & 0.90400 & -0.47500 \\
\hline-5578.406 & 51.875 & 59.998 & 38.555 & 0.05120 & 0.85400 & -0.51800 \\
\hline-5574.391 & 51.875 & 60.076 & 39.789 & 0.02020 & 0.80400 & -0.57000 \\
\hline-5570.377 & 51.875 & 60.064 & 40.844 & -0.01310 & 0.75200 & -0.61800 \\
\hline-5566.363 & 51.875 & 59.997 & 41.418 & -0.04800 & 0.69600 & -0.65500 \\
\hline-5562.349 & 51.625 & 59.838 & 41.450 & -0.08190 & 0.63700 & -0.68700 \\
\hline-5558.335 & 51.375 & 59.642 & 41.117 & -0.1121 & 0.57800 & -0.71600 \\
\hline-5554.320 & 50.875 & 59.395 & 40.479 & -0.1380 & 0.51800 & -0.73200 \\
\hline-5550.306 & 50.125 & 59.132 & 39.854 & -0.1597 & 0.46300 & -0.74500 \\
\hline-5546.292 & 49.375 & 58.818 & 39.310 & -0.1771 & 0.41300 & -0.74300 \\
\hline-5542.278 & 48.375 & 58.513 & 38.767 & -0.1902 & 0.36800 & -0.73100 \\
\hline-5538.263 & 47.625 & 58.160 & 38.191 & -0.1991 & 0.33500 & -0.71100 \\
\hline-5534.249 & 46.875 & 57.727 & 37.594 & -0.2037 & 0.30700 & -0.69200 \\
\hline-5530.235 & 45.875 & 57.245 & 36.989 & -0.2045 & 0.29200 & -0.66000 \\
\hline-5526.221 & 45.125 & 56.689 & 36.477 & -0.2032 & 0.27300 & -0.63100 \\
\hline-5522.206 & 44.375 & 56.009 & 35.895 & -0.2020 & 0.25600 & -0.60500 \\
\hline-5518.192 & 43.625 & 55.284 & 35.344 & -0.2011 & 0.24100 & -0.58000 \\
\hline-5514.178 & 42.875 & 54.450 & 34.753 & -0.2005 & 0.21100 & -0.56600 \\
\hline-5510.164 & 42.125 & 53.438 & 34.068 & -0.2003 & 0.17800 & -0.55500 \\
\hline-5506.149 & 41.375 & 52.338 & 33.257 & -0.2004 & 0.14900 & -0.54500 \\
\hline-5502.135 & 40.625 & 51.096 & 32.307 & -0.2008 & 0.12000 & -0.53300 \\
\hline-5498.121 & 39.875 & 49.820 & 31.290 & -0.2016 & 0.086000 & -0.52300 \\
\hline-5494.107 & 39.125 & 48.627 & 30.375 & -0.2025 & 0.065000 & -0.51500 \\
\hline-5490.093 & 38.375 & 47.524 & 29.755 & -0.2023 & 0.062000 & -0.50500 \\
\hline-5486.078 & 37.625 & 46.470 & 29.327 & -0.1999 & 0.061000 & -0.49300 \\
\hline-5482.064 & 36.875 & 45.397 & 29.008 & -0.1950 & 0.063000 & -0.48200 \\
\hline-5478.050 & 36.125 & 44.355 & 28.685 & -0.1876 & 0.064000 & -0.46800 \\
\hline-5474.036 & 35.375 & 43.341 & 28.438 & -0.1777 & 0.066000 & -0.45800 \\
\hline-5470.021 & 34.875 & 42.333 & 28.224 & -0.1653 & 0.070000 & -0.44200 \\
\hline-5466.007 & 34.125 & 41.373 & 28.048 & -0.1504 & 0.074000 & -0.42300 \\
\hline-5461.993 & 33.375 & 40.445 & 27.905 & -0.1333 & 0.080000 & -0.40500 \\
\hline-5457.979 & 32.875 & 39.607 & 27.771 & -0.1154 & 0.087000 & -0.38200 \\
\hline-5453.964 & 32.125 & 38.791 & 27.646 & -0.09810 & 0.094000 & -0.36300 \\
\hline-5449.950 & 31.625 & 37.965 & 27.587 & -0.08170 & 0.10100 & -0.34600 \\
\hline-5445.936 & 31.375 & 37.206 & 27.534 & -0.06620 & 0.10800 & -0.32500 \\
\hline-5441.922 & 31.125 & 36.464 & 27.478 & -0.05160 & 0.11600 & -0.30800 \\
\hline-5437.907 & 30.875 & 35.784 & 27.444 & -0.03790 & 0.12500 & -0.29200 \\
\hline-5433.893 & 30.875 & 35.105 & 27.419 & -0.02500 & 0.13300 & -0.27700 \\
\hline-5429.879 & 30.875 & 34.507 & 27.380 & -0.01310 & 0.14300 & -0.25900 \\
\hline-5425.865 & 30.625 & 33.995 & 27.350 & -0.002100 & 0.15200 & -0.24200 \\
\hline-5421.851 & 30.625 & 33.679 & 27.365 & 0.008100 & 0.16400 & -0.22600 \\
\hline-5417.836 & 30.875 & 33.638 & 27.355 & 0.01740 & 0.17200 & -0.21000 \\
\hline-5413.822 & 30.875 & 33.807 & 27.349 & 0.02580 & 0.18300 & -0.19400 \\
\hline-5409.808 & 30.875 & 34.076 & 27.345 & 0.03320 & 0.19600 & -0.18000 \\
\hline-5405.794 & 31.125 & 34.387 & 27.332 & 0.03980 & 0.20900 & -0.16600 \\
\hline-5401.779 & 31.375 & 34.744 & 27.363 & 0.04550 & 0.22400 & -0.15500 \\
\hline-5397.765 & 31.625 & 35.133 & 27.396 & 0.05030 & 0.23900 & -0.14400 \\
\hline-5393.751 & 31.875 & 35.557 & 27.431 & 0.05430 & 0.25300 & -0.13300 \\
\hline
\end{tabular}




\begin{tabular}{|c|c|c|c|c|c|c|}
\hline-5389.737 & 32.125 & 36.040 & 27.529 & 0.05740 & 0.26800 & -0.12300 \\
\hline-5385.722 & 32.375 & 36.543 & 27.634 & 0.05990 & 0.28200 & -0.11100 \\
\hline-5381.708 & 32.625 & 37.103 & 27.811 & 0.06210 & 0.29200 & -0.098000 \\
\hline-5377.694 & 33.125 & 37.620 & 27.990 & 0.06420 & 0.30200 & -0.087000 \\
\hline-5373.680 & 33.375 & 38.161 & 28.293 & 0.06600 & 0.31000 & -0.077000 \\
\hline-5369.665 & 33.625 & 38.689 & 28.682 & 0.06760 & 0.30900 & -0.068000 \\
\hline-5365.651 & 33.875 & 39.183 & 29.161 & 0.06900 & 0.30800 & -0.057000 \\
\hline-5361.637 & 34.125 & 39.678 & 29.663 & 0.07020 & 0.30100 & -0.050000 \\
\hline-5357.623 & 34.375 & 40.102 & 30.211 & 0.07110 & 0.29200 & -0.044000 \\
\hline-5353.609 & 34.875 & 40.438 & 30.818 & 0.07190 & 0.27900 & -0.040000 \\
\hline-5349.594 & 35.125 & 40.670 & 31.415 & 0.07240 & 0.26100 & -0.037000 \\
\hline-5345.580 & 35.375 & 40.816 & 31.957 & 0.07270 & 0.24100 & -0.037000 \\
\hline-5341.566 & 35.625 & 40.909 & 32.464 & 0.07270 & 0.22100 & -0.038000 \\
\hline-5337.552 & 35.875 & 40.949 & 32.909 & 0.07260 & 0.20000 & -0.040000 \\
\hline-5333.537 & 36.125 & 40.938 & 33.320 & 0.07230 & 0.18300 & -0.042000 \\
\hline-5329.523 & 36.375 & 40.926 & 33.711 & 0.07170 & 0.16700 & -0.044000 \\
\hline-5325.509 & 36.625 & 40.844 & 34.069 & 0.07090 & 0.15200 & -0.046000 \\
\hline-5321.495 & 36.875 & 40.806 & 34.400 & 0.06990 & 0.14100 & -0.047000 \\
\hline-5317.480 & 37.125 & 40.798 & 34.720 & 0.06880 & 0.13200 & -0.047000 \\
\hline-5313.466 & 37.375 & 40.815 & 35.025 & 0.06780 & 0.12500 & -0.045000 \\
\hline-5309.452 & 37.625 & 40.855 & 35.322 & 0.06670 & 0.12000 & -0.044000 \\
\hline-5305.438 & 37.875 & 40.923 & 35.605 & 0.06580 & 0.11500 & -0.044000 \\
\hline-5301.423 & 38.125 & 41.009 & 35.880 & 0.06480 & 0.11200 & -0.043000 \\
\hline-5297.409 & 38.375 & 41.123 & 36.149 & 0.06390 & 0.10900 & -0.041000 \\
\hline-5293.395 & 38.625 & 41.263 & 36.404 & 0.06300 & 0.10700 & -0.040000 \\
\hline-5289.381 & 38.875 & 41.427 & 36.653 & 0.06220 & 0.10500 & -0.039000 \\
\hline-5285.367 & 39.125 & 41.613 & 36.887 & 0.06140 & 0.10400 & -0.038000 \\
\hline-5281.352 & 39.375 & 41.808 & 37.106 & 0.06070 & 0.10300 & -0.036000 \\
\hline-5277.338 & 39.625 & 42.015 & 37.317 & 0.06000 & 0.10200 & -0.034000 \\
\hline-5273.324 & 39.875 & 42.237 & 37.517 & 0.05930 & 0.10100 & -0.031000 \\
\hline-5269.310 & 40.125 & 42.468 & 37.698 & 0.05870 & 0.10100 & -0.029000 \\
\hline-5265.295 & 40.375 & 42.711 & 37.863 & 0.05810 & 0.10000 & -0.026000 \\
\hline-5261.281 & 40.625 & 42.963 & 38.016 & 0.05750 & 0.10000 & -0.023000 \\
\hline-5257.267 & 40.875 & 43.218 & 38.164 & 0.05700 & 0.10000 & -0.020000 \\
\hline-5253.253 & 40.875 & 43.486 & 38.287 & 0.05660 & 0.10100 & -0.016000 \\
\hline-5249.238 & 41.125 & 43.756 & 38.411 & 0.05610 & 0.10100 & -0.011000 \\
\hline-5245.224 & 41.375 & 44.029 & 38.535 & 0.05570 & 0.10200 & -0.0080000 \\
\hline-5241.210 & 41.625 & 44.305 & 38.677 & 0.05540 & 0.10300 & -0.0040000 \\
\hline-5237.196 & 41.875 & 44.581 & 38.835 & 0.05510 & 0.10400 & -0.0010000 \\
\hline-5233.181 & 42.125 & 44.856 & 38.979 & 0.05480 & 0.10500 & 0.0010000 \\
\hline-5229.167 & 42.375 & 45.130 & 39.171 & 0.05460 & 0.10700 & 0.0030000 \\
\hline-5225.153 & 42.625 & 45.400 & 39.372 & 0.05440 & 0.10800 & 0.0040000 \\
\hline-5221.139 & 42.875 & 45.682 & 39.587 & 0.05420 & 0.10900 & 0.0040000 \\
\hline-5217.125 & 43.125 & 45.971 & 39.826 & 0.05410 & 0.11000 & 0.0040000 \\
\hline-5213.110 & 43.375 & 46.255 & 40.055 & 0.05400 & 0.11000 & 0.0020000 \\
\hline-5209.096 & 43.625 & 46.544 & 40.296 & 0.05400 & 0.11100 & 0.0010000 \\
\hline-5205.082 & 43.875 & 46.843 & 40.543 & 0.05400 & 0.11200 & 0.0000 \\
\hline-5201.068 & 44.125 & 47.148 & 40.783 & 0.05400 & 0.11300 & -0.0020000 \\
\hline-5197.053 & 44.375 & 47.443 & 41.022 & 0.05410 & 0.11300 & -0.0060000 \\
\hline-5193.039 & 44.625 & 47.746 & 41.259 & 0.05420 & 0.11400 & -0.0080000 \\
\hline-5189.025 & 44.625 & 48.051 & 41.479 & 0.05430 & 0.11400 & -0.011000 \\
\hline-5185.011 & 44.875 & 48.362 & 41.695 & 0.05450 & 0.11600 & -0.014000 \\
\hline-5180.996 & 45.125 & 48.658 & 41.903 & 0.05480 & 0.11800 & -0.018000 \\
\hline-5176.982 & 45.375 & 48.958 & 42.105 & 0.05500 & 0.11900 & -0.023000 \\
\hline
\end{tabular}




\begin{tabular}{|c|c|c|c|c|c|c|}
\hline-5172.968 & 45.625 & 49.271 & 42.294 & 0.05520 & 0.12100 & -0.028000 \\
\hline-5168.954 & 45.875 & 49.599 & 42.463 & 0.05540 & 0.12300 & -0.033000 \\
\hline-5164.940 & 46.125 & 49.933 & 42.636 & 0.05550 & 0.12500 & -0.037000 \\
\hline-5160.925 & 46.125 & 50.267 & 42.800 & 0.05560 & 0.12800 & -0.043000 \\
\hline-5156.911 & 46.375 & 50.615 & 42.940 & 0.05570 & 0.13000 & -0.049000 \\
\hline-5152.897 & 46.625 & 50.935 & 43.075 & 0.05570 & 0.13300 & -0.053000 \\
\hline-5148.883 & 46.875 & 51.270 & 43.214 & 0.05570 & 0.13600 & -0.058000 \\
\hline-5144.868 & 47.125 & 51.606 & 43.341 & 0.05560 & 0.14000 & -0.063000 \\
\hline-5140.854 & 47.375 & 51.948 & 43.467 & 0.05560 & 0.14500 & -0.067000 \\
\hline-5136.840 & 47.375 & 52.296 & 43.579 & 0.05540 & 0.14900 & -0.073000 \\
\hline-5132.826 & 47.625 & 52.643 & 43.707 & 0.05530 & 0.15600 & -0.078000 \\
\hline-5128.811 & 47.875 & 52.977 & 43.822 & 0.05510 & 0.16300 & -0.084000 \\
\hline-5124.797 & 48.125 & 53.324 & 43.907 & 0.05490 & 0.16900 & -0.091000 \\
\hline-5120.783 & 48.375 & 53.673 & 43.996 & 0.05460 & 0.17700 & -0.099000 \\
\hline-5116.769 & 48.625 & 54.050 & 44.079 & 0.05430 & 0.18200 & -0.10800 \\
\hline-5112.754 & 48.875 & 54.397 & 44.166 & 0.05400 & 0.18800 & -0.11400 \\
\hline-5108.740 & 48.875 & 54.775 & 44.234 & 0.05370 & 0.19500 & -0.12200 \\
\hline-5104.726 & 49.125 & 55.125 & 44.276 & 0.05330 & 0.20200 & -0.13000 \\
\hline-5100.712 & 49.375 & 55.488 & 44.302 & 0.05280 & 0.20800 & -0.13900 \\
\hline-5096.698 & 49.625 & 55.823 & 44.274 & 0.05240 & 0.21400 & -0.14800 \\
\hline-5092.683 & 49.875 & 56.134 & 44.252 & 0.05190 & 0.21900 & -0.15700 \\
\hline-5088.669 & 50.125 & 56.476 & 44.180 & 0.05130 & 0.22400 & -0.17100 \\
\hline-5084.655 & 50.375 & 56.774 & 44.081 & 0.05080 & 0.23100 & -0.18400 \\
\hline-5080.641 & 50.375 & 57.080 & 43.946 & 0.05020 & 0.23500 & -0.19500 \\
\hline-5076.626 & 50.625 & 57.400 & 43.841 & 0.04950 & 0.23900 & -0.21000 \\
\hline-5072.612 & 50.875 & 57.700 & 43.634 & 0.04890 & 0.24400 & -0.22200 \\
\hline-5068.598 & 51.125 & 57.980 & 43.456 & 0.04820 & 0.25000 & -0.23300 \\
\hline-5064.584 & 51.375 & 58.301 & 43.232 & 0.04740 & 0.25200 & -0.24500 \\
\hline-5060.569 & 51.375 & 58.593 & 42.995 & 0.04660 & 0.25300 & -0.26300 \\
\hline-5056.555 & 51.625 & 58.881 & 42.710 & 0.04580 & 0.25700 & -0.27700 \\
\hline-5052.541 & 51.875 & 59.106 & 42.378 & 0.04500 & 0.26100 & -0.29200 \\
\hline-5048.527 & 52.125 & 59.385 & 42.064 & 0.04410 & 0.26900 & -0.30400 \\
\hline-5044.512 & 52.125 & 59.618 & 41.751 & 0.04320 & 0.27100 & -0.31700 \\
\hline-5040.498 & 52.375 & 59.894 & 41.365 & 0.04220 & 0.27600 & -0.33500 \\
\hline-5036.484 & 52.625 & 60.150 & 40.834 & 0.04120 & 0.28100 & -0.34600 \\
\hline-5032.470 & 52.625 & 60.406 & 40.369 & 0.04000 & 0.28700 & -0.36100 \\
\hline-5028.456 & 52.875 & 60.618 & 39.751 & 0.03880 & 0.29200 & -0.37600 \\
\hline-5024.441 & 53.125 & 60.900 & 39.274 & 0.03740 & 0.29600 & -0.39100 \\
\hline-5020.427 & 53.125 & 61.147 & 38.694 & 0.03590 & 0.30200 & -0.41200 \\
\hline-5016.413 & 53.375 & 61.328 & 38.123 & 0.03440 & 0.30500 & -0.43200 \\
\hline-5012.399 & 53.375 & 61.555 & 37.514 & 0.03270 & 0.31100 & -0.44900 \\
\hline-5008.384 & 53.625 & 61.761 & 36.933 & 0.03080 & 0.31600 & -0.46200 \\
\hline-5004.370 & 53.625 & 61.935 & 36.316 & 0.02890 & 0.32500 & -0.47400 \\
\hline-5000.356 & 53.875 & 62.137 & 35.744 & 0.02690 & 0.33200 & -0.48800 \\
\hline-4996.342 & 53.875 & 62.323 & 35.155 & 0.02470 & 0.34000 & -0.50000 \\
\hline-4992.327 & 54.125 & 62.509 & 34.561 & 0.02250 & 0.34600 & -0.51800 \\
\hline-4988.313 & 54.125 & 62.682 & 34.047 & 0.02010 & 0.35700 & -0.53200 \\
\hline-4984.299 & 54.125 & 62.881 & 33.508 & 0.01760 & 0.36700 & -0.54700 \\
\hline-4980.285 & 54.125 & 63.077 & 33.020 & 0.01500 & 0.37800 & -0.55800 \\
\hline-4976.270 & 54.375 & 63.265 & 32.577 & 0.01230 & 0.38700 & -0.56900 \\
\hline-4972.256 & 54.375 & 63.448 & 32.210 & 0.009500 & 0.39800 & -0.57800 \\
\hline-4968.242 & 54.375 & 63.612 & 31.791 & 0.006500 & 0.40700 & -0.59300 \\
\hline-4964.228 & 54.375 & 63.819 & 31.397 & 0.003500 & 0.42000 & -0.60700 \\
\hline-4960.214 & 54.375 & 64.031 & 31.069 & 0.0003000 & 0.42500 & -0.62700 \\
\hline
\end{tabular}




$\begin{array}{lllllll}-4956.199 & 54.375 & 64.232 & 30.709 & -0.003000 & 0.43800 & -0.64400 \\ -4952.185 & 54.375 & 64.430 & 30.269 & -0.006300 & 0.45500 & -0.66200 \\ -4948.171 & 54.375 & 64.631 & 29.963 & -0.009800 & 0.47600 & -0.68400 \\ -4944.157 & 54.375 & 64.818 & 29.726 & -0.01350 & 0.49900 & -0.70900 \\ -4940.142 & 54.125 & 65.031 & 29.440 & -0.01720 & 0.52400 & -0.74300 \\ -4936.128 & 54.125 & 65.255 & 29.164 & -0.02100 & 0.55600 & -0.77600 \\ -4932.114 & 54.125 & 65.459 & 28.849 & -0.02500 & 0.59400 & -0.81700 \\ -4928.100 & 53.875 & 65.675 & 28.479 & -0.02900 & 0.63000 & -0.87400 \\ -4924.085 & 53.625 & 65.904 & 28.042 & -0.03320 & 0.69000 & -0.94900 \\ -4920.071 & 53.625 & 66.169 & 27.616 & -0.03750 & 0.76500 & -1.0400 \\ -4916.057 & 53.375 & 66.421 & 27.110 & -0.04190 & 0.86700 & -1.1620 \\ -4912.043 & 53.125 & 66.732 & 26.456 & -0.04640 & 1.0190 & -1.3580 \\ -4908.028 & 53.125 & 67.105 & 25.631 & -0.05100 & 1.2270 & -1.6630 \\ -4904.014 & 52.875 & 67.569 & 24.401 & -0.05570 & 1.6130 & -2.2590 \\ -4900.000 & 53.125 & 68.624 & 21.906 & -0.06050 & 2.5990 & -3.9950\end{array}$




\begin{tabular}{|c|c|c|c|c|c|c|}
\hline \multicolumn{7}{|l|}{ Figure $54 a, b$} \\
\hline Date & Median (muT) & Cred_Int Upper & Cred_Int Lower & dFdt (muT/yr) & dFdt Upper & dFdt Lower \\
\hline-7000.000 & 55.125 & 68.569 & 22.224 & 0.0169 & 1.9560 & -1.0510 \\
\hline-6995.992 & 54.875 & 67.889 & 24.083 & 0.0162 & 1.4180 & -0.83000 \\
\hline-6991.984 & 54.875 & 67.557 & 25.067 & 0.0156 & 1.1270 & -0.68900 \\
\hline-6987.976 & 55.125 & 67.321 & 25.848 & 0.0149 & 0.95600 & -0.60400 \\
\hline-6983.968 & 55.125 & 67.113 & 26.476 & 0.0142 & 0.84000 & -0.53600 \\
\hline-6979.959 & 55.125 & 66.935 & 26.992 & 0.0135 & 0.76200 & -0.48600 \\
\hline-6975.951 & 55.125 & 66.777 & 27.468 & 0.0129 & 0.68900 & -0.44900 \\
\hline-6971.943 & 55.125 & 66.620 & 27.867 & 0.0122 & 0.63800 & -0.41300 \\
\hline-6967.935 & 55.125 & 66.470 & 28.245 & 0.0115 & 0.59000 & -0.39000 \\
\hline-6963.927 & 55.375 & 66.337 & 28.541 & 0.0109 & 0.55100 & -0.36700 \\
\hline-6959.919 & 55.375 & 66.206 & 28.821 & 0.0102 & 0.51900 & -0.34800 \\
\hline-6955.911 & 55.375 & 66.060 & 29.101 & 0.00960 & 0.49100 & -0.33000 \\
\hline-6951.903 & 55.375 & 65.950 & 29.340 & 0.00900 & 0.47000 & -0.31600 \\
\hline-6947.894 & 55.375 & 65.832 & 29.615 & 0.00830 & 0.45100 & -0.30300 \\
\hline-6943.886 & 55.625 & 65.704 & 29.869 & 0.00770 & 0.43200 & -0.29100 \\
\hline-6939.878 & 55.625 & 65.582 & 30.062 & 0.00710 & 0.41500 & -0.28200 \\
\hline-6935.870 & 55.625 & 65.471 & 30.324 & 0.00650 & 0.40100 & -0.27100 \\
\hline-6931.862 & 55.625 & 65.354 & 30.520 & 0.00590 & 0.38800 & -0.26400 \\
\hline-6927.854 & 55.625 & 65.253 & 30.667 & 0.00530 & 0.37600 & -0.25500 \\
\hline-6923.846 & 55.625 & 65.149 & 30.873 & 0.00470 & 0.36500 & -0.24800 \\
\hline-6919.838 & 55.625 & 65.031 & 31.061 & 0.00410 & 0.35500 & -0.24200 \\
\hline-6915.830 & 55.625 & 64.921 & 31.223 & 0.00350 & 0.34600 & -0.23600 \\
\hline-6911.821 & 55.625 & 64.823 & 31.413 & 0.00290 & 0.33700 & -0.23100 \\
\hline-6907.813 & 55.625 & 64.711 & 31.557 & 0.00230 & 0.32900 & -0.22500 \\
\hline-6903.805 & 55.625 & 64.620 & 31.711 & 0.00180 & 0.32200 & -0.21800 \\
\hline-6899.797 & 55.625 & 64.523 & 31.899 & 0.00120 & 0.31600 & -0.21400 \\
\hline-6895.789 & 55.625 & 64.424 & 32.054 & 0.000700 & 0.31000 & -0.20900 \\
\hline-6891.781 & 55.625 & 64.312 & 32.246 & $1.00 \mathrm{e}-04$ & 0.30600 & -0.20300 \\
\hline-6887.773 & 55.625 & 64.204 & 32.481 & -0.000400 & 0.30200 & -0.19700 \\
\hline-6883.765 & 55.625 & 64.103 & 32.666 & -0.00100 & 0.29800 & -0.19300 \\
\hline-6879.757 & 55.625 & 63.999 & 32.898 & -0.00150 & 0.29400 & -0.18800 \\
\hline-6875.748 & 55.625 & 63.918 & 33.111 & -0.00200 & 0.29000 & -0.18400 \\
\hline-6871.740 & 55.625 & 63.840 & 33.355 & -0.00260 & 0.28600 & -0.18100 \\
\hline-6867.732 & 55.625 & 63.731 & 33.596 & -0.00310 & 0.28200 & -0.17700 \\
\hline-6863.724 & 55.625 & 63.659 & 33.814 & -0.00360 & 0.28000 & -0.17400 \\
\hline-6859.716 & 55.625 & 63.567 & 34.059 & -0.00410 & 0.27700 & -0.17000 \\
\hline-6855.708 & 55.625 & 63.474 & 34.311 & -0.00460 & 0.27200 & -0.16700 \\
\hline-6851.700 & 55.625 & 63.371 & 34.551 & -0.00510 & 0.27000 & -0.16300 \\
\hline-6847.692 & 55.625 & 63.288 & 34.806 & -0.00560 & 0.26700 & -0.15900 \\
\hline-6843.683 & 55.625 & 63.192 & 35.102 & -0.00610 & 0.26300 & -0.15500 \\
\hline-6839.675 & 55.625 & 63.096 & 35.403 & -0.00660 & 0.26000 & -0.15200 \\
\hline-6835.667 & 55.625 & 63.040 & 35.744 & -0.00700 & 0.25900 & -0.15000 \\
\hline-6831.659 & 55.625 & 62.974 & 36.039 & -0.00750 & 0.25500 & -0.14600 \\
\hline-6827.651 & 55.375 & 62.881 & 36.389 & -0.00800 & 0.25100 & -0.14300 \\
\hline-6823.643 & 55.375 & 62.775 & 36.639 & -0.00840 & 0.24600 & -0.14000 \\
\hline-6819.635 & 55.375 & 62.677 & 37.012 & -0.00890 & 0.24300 & -0.13800 \\
\hline-6815.627 & 55.375 & 62.572 & 37.307 & -0.00930 & 0.23900 & -0.13500 \\
\hline-6811.619 & 55.375 & 62.470 & 37.647 & -0.00980 & 0.23400 & -0.13300 \\
\hline-6807.610 & 55.375 & 62.352 & 37.997 & -0.0102 & 0.23100 & -0.13000 \\
\hline-6803.602 & 55.125 & 62.243 & 38.443 & -0.0106 & 0.22900 & -0.12700 \\
\hline-6799.594 & 55.125 & 62.144 & 38.758 & -0.0111 & 0.22700 & -0.12400 \\
\hline
\end{tabular}




\begin{tabular}{|c|c|c|c|c|c|c|}
\hline-6795.586 & 55.125 & 62.008 & 39.189 & -0.0115 & 0.22200 & -0.12300 \\
\hline-6791.578 & 55.125 & 61.912 & 39.644 & -0.0119 & 0.21800 & -0.12000 \\
\hline-6787.570 & 55.125 & 61.793 & 40.107 & -0.0123 & 0.21300 & -0.11800 \\
\hline-6783.562 & 55.125 & 61.665 & 40.627 & -0.0127 & 0.20800 & -0.11700 \\
\hline-6779.554 & 54.875 & 61.531 & 41.064 & -0.0131 & 0.20400 & -0.11400 \\
\hline-6775.545 & 54.875 & 61.381 & 41.507 & -0.0135 & 0.20000 & -0.11300 \\
\hline-6771.537 & 54.875 & 61.229 & 41.950 & -0.0139 & 0.19700 & -0.11000 \\
\hline-6767.529 & 54.875 & 61.086 & 42.396 & -0.0143 & 0.19100 & -0.11000 \\
\hline-6763.521 & 54.625 & 60.933 & 42.900 & -0.0147 & 0.18700 & -0.10800 \\
\hline-6759.513 & 54.625 & 60.765 & 43.410 & -0.0150 & 0.18200 & -0.10500 \\
\hline-6755.505 & 54.625 & 60.605 & 43.888 & -0.0154 & 0.17900 & -0.10300 \\
\hline-6751.497 & 54.625 & 60.444 & 44.458 & -0.0157 & 0.17200 & -0.10300 \\
\hline-6747.489 & 54.375 & 60.277 & 44.955 & -0.0161 & 0.16600 & -0.10100 \\
\hline-6743.481 & 54.375 & 60.096 & 45.511 & -0.0164 & 0.16000 & -0.10000 \\
\hline-6739.472 & 54.375 & 59.905 & 45.989 & -0.0168 & 0.15500 & -0.099000 \\
\hline-6735.464 & 54.375 & 59.726 & 46.443 & -0.0171 & 0.14800 & -0.098000 \\
\hline-6731.456 & 54.125 & 59.536 & 46.913 & -0.0175 & 0.14200 & -0.097000 \\
\hline-6727.448 & 54.125 & 59.346 & 47.303 & -0.0178 & 0.13500 & -0.097000 \\
\hline-6723.440 & 54.125 & 59.169 & 47.678 & -0.0181 & 0.12900 & -0.097000 \\
\hline-6719.432 & 53.875 & 58.994 & 48.053 & -0.0184 & 0.12400 & -0.096000 \\
\hline-6715.424 & 53.875 & 58.825 & 48.327 & -0.0187 & 0.11900 & -0.096000 \\
\hline-6711.416 & 53.875 & 58.662 & 48.538 & -0.0190 & 0.11400 & -0.096000 \\
\hline-6707.408 & 53.875 & 58.480 & 48.702 & -0.0193 & 0.10800 & -0.096000 \\
\hline-6703.399 & 53.625 & 58.324 & 48.801 & -0.0196 & 0.10300 & -0.096000 \\
\hline-6699.391 & 53.625 & 58.164 & 48.897 & -0.0199 & 0.098000 & -0.095000 \\
\hline-6695.383 & 53.625 & 58.019 & 48.931 & -0.0202 & 0.094000 & -0.095000 \\
\hline-6691.375 & 53.375 & 57.903 & 48.968 & -0.0205 & 0.089000 & -0.095000 \\
\hline-6687.367 & 53.375 & 57.773 & 48.957 & -0.0208 & 0.085000 & -0.095000 \\
\hline-6683.359 & 53.375 & 57.673 & 48.917 & -0.0210 & 0.081000 & -0.095000 \\
\hline-6679.351 & 53.125 & 57.559 & 48.876 & -0.0213 & 0.077000 & -0.094000 \\
\hline-6675.343 & 53.125 & 57.472 & 48.825 & -0.0215 & 0.074000 & -0.094000 \\
\hline-6671.334 & 53.125 & 57.394 & 48.756 & -0.0218 & 0.072000 & -0.094000 \\
\hline-6667.326 & 52.875 & 57.315 & 48.663 & -0.0220 & 0.069000 & -0.094000 \\
\hline-6663.318 & 52.875 & 57.248 & 48.579 & -0.0223 & 0.067000 & -0.093000 \\
\hline-6659.310 & 52.625 & 57.184 & 48.498 & -0.0225 & 0.065000 & -0.094000 \\
\hline-6655.302 & 52.625 & 57.144 & 48.400 & -0.0227 & 0.064000 & -0.094000 \\
\hline-6651.294 & 52.625 & 57.108 & 48.276 & -0.0229 & 0.062000 & -0.094000 \\
\hline-6647.286 & 52.375 & 57.089 & 48.150 & -0.0232 & 0.060000 & -0.094000 \\
\hline-6643.278 & 52.375 & 57.073 & 47.999 & -0.0234 & 0.060000 & -0.094000 \\
\hline-6639.270 & 52.375 & 57.052 & 47.855 & -0.0236 & 0.059000 & -0.094000 \\
\hline-6635.261 & 52.125 & 57.023 & 47.703 & -0.0238 & 0.059000 & -0.094000 \\
\hline-6631.253 & 52.125 & 57.037 & 47.528 & -0.0240 & 0.059000 & -0.094000 \\
\hline-6627.245 & 51.875 & 57.038 & 47.345 & -0.0242 & 0.058000 & -0.094000 \\
\hline-6623.237 & 51.875 & 57.050 & 47.158 & -0.0243 & 0.059000 & -0.094000 \\
\hline-6619.229 & 51.875 & 57.063 & 46.974 & -0.0245 & 0.059000 & -0.094000 \\
\hline-6615.221 & 51.625 & 57.075 & 46.779 & -0.0247 & 0.060000 & -0.094000 \\
\hline-6611.213 & 51.625 & 57.100 & 46.598 & -0.0249 & 0.061000 & -0.094000 \\
\hline-6607.205 & 51.375 & 57.129 & 46.384 & -0.0250 & 0.061000 & -0.096000 \\
\hline-6603.196 & 51.375 & 57.138 & 46.190 & -0.0252 & 0.062000 & -0.096000 \\
\hline-6599.188 & 51.375 & 57.156 & 45.986 & -0.0253 & 0.062000 & -0.097000 \\
\hline-6595.180 & 51.125 & 57.172 & 45.772 & -0.0255 & 0.063000 & -0.098000 \\
\hline-6591.172 & 51.125 & 57.212 & 45.568 & -0.0256 & 0.064000 & -0.098000 \\
\hline-6587.164 & 50.875 & 57.243 & 45.371 & -0.0258 & 0.066000 & -0.10000 \\
\hline-6583.156 & 50.875 & 57.251 & 45.131 & -0.0259 & 0.067000 & -0.10100 \\
\hline
\end{tabular}




\begin{tabular}{|c|c|c|c|c|c|c|}
\hline-6579.148 & 50.875 & 57.269 & 44.933 & -0.0260 & 0.068000 & -0.10300 \\
\hline-6575.140 & 50.625 & 57.285 & 44.733 & -0.0261 & 0.069000 & -0.10400 \\
\hline-6571.132 & 50.625 & 57.280 & 44.514 & -0.0262 & 0.071000 & -0.10500 \\
\hline-6567.123 & 50.375 & 57.277 & 44.325 & -0.0263 & 0.072000 & -0.10700 \\
\hline-6563.115 & 50.375 & 57.286 & 44.105 & -0.0264 & 0.073000 & -0.10900 \\
\hline-6559.107 & 50.375 & 57.302 & 43.880 & -0.0265 & 0.075000 & -0.11000 \\
\hline-6555.099 & 50.125 & 57.316 & 43.636 & -0.0266 & 0.076000 & -0.11200 \\
\hline-6551.091 & 50.125 & 57.324 & 43.373 & -0.0267 & 0.077000 & -0.11400 \\
\hline-6547.083 & 49.875 & 57.366 & 43.137 & -0.0268 & 0.079000 & -0.11600 \\
\hline-6543.075 & 49.875 & 57.370 & 42.907 & -0.0269 & 0.081000 & -0.11800 \\
\hline-6539.067 & 49.625 & 57.374 & 42.683 & -0.0269 & 0.081000 & -0.12000 \\
\hline-6535.059 & 49.625 & 57.368 & 42.430 & -0.0270 & 0.083000 & -0.12300 \\
\hline-6531.050 & 49.625 & 57.350 & 42.181 & -0.0271 & 0.085000 & -0.12400 \\
\hline-6527.042 & 49.375 & 57.362 & 41.940 & -0.0271 & 0.086000 & -0.12700 \\
\hline-6523.034 & 49.375 & 57.362 & 41.723 & -0.0272 & 0.088000 & -0.12800 \\
\hline-6519.026 & 49.125 & 57.355 & 41.528 & -0.0272 & 0.089000 & -0.13100 \\
\hline-6515.018 & 49.125 & 57.352 & 41.368 & -0.0272 & 0.090000 & -0.13300 \\
\hline-6511.010 & 48.875 & 57.334 & 41.162 & -0.0273 & 0.090000 & -0.13500 \\
\hline-6507.002 & 48.875 & 57.298 & 40.996 & -0.0273 & 0.091000 & -0.13700 \\
\hline-6502.994 & 48.875 & 57.259 & 40.821 & -0.0273 & 0.092000 & -0.13900 \\
\hline-6498.985 & 48.625 & 57.248 & 40.675 & -0.0273 & 0.093000 & -0.14100 \\
\hline-6494.977 & 48.625 & 57.227 & 40.533 & -0.0273 & 0.094000 & -0.14400 \\
\hline-6490.969 & 48.375 & 57.173 & 40.381 & -0.0274 & 0.094000 & -0.14600 \\
\hline-6486.961 & 48.375 & 57.156 & 40.250 & -0.0274 & 0.095000 & -0.14700 \\
\hline-6482.953 & 48.125 & 57.152 & 40.140 & -0.0275 & 0.095000 & -0.14900 \\
\hline-6478.945 & 48.125 & 57.121 & 40.046 & -0.0276 & 0.096000 & -0.15100 \\
\hline-6474.937 & 47.875 & 57.069 & 39.928 & -0.0278 & 0.096000 & -0.15400 \\
\hline-6470.929 & 47.875 & 57.021 & 39.823 & -0.0279 & 0.097000 & -0.15600 \\
\hline-6466.921 & 47.625 & 56.986 & 39.727 & -0.0281 & 0.097000 & -0.15800 \\
\hline-6462.912 & 47.625 & 56.950 & 39.624 & -0.0283 & 0.097000 & -0.16100 \\
\hline-6458.904 & 47.375 & 56.889 & 39.538 & -0.0285 & 0.096000 & -0.16400 \\
\hline-6454.896 & 47.375 & 56.837 & 39.452 & -0.0287 & 0.097000 & -0.16600 \\
\hline-6450.888 & 47.375 & 56.773 & 39.373 & -0.0289 & 0.097000 & -0.16800 \\
\hline-6446.880 & 47.125 & 56.702 & 39.310 & -0.0292 & 0.097000 & -0.17000 \\
\hline-6442.872 & 47.125 & 56.605 & 39.185 & -0.0295 & 0.098000 & -0.17300 \\
\hline-6438.864 & 46.875 & 56.541 & 39.089 & -0.0298 & 0.098000 & -0.17500 \\
\hline-6434.856 & 46.875 & 56.467 & 38.991 & -0.0301 & 0.098000 & -0.17800 \\
\hline-6430.847 & 46.625 & 56.374 & 38.896 & -0.0305 & 0.097000 & -0.18000 \\
\hline-6426.839 & 46.625 & 56.288 & 38.800 & -0.0309 & 0.096000 & -0.18400 \\
\hline-6422.831 & 46.375 & 56.188 & 38.710 & -0.0313 & 0.095000 & -0.18500 \\
\hline-6418.823 & 46.375 & 56.105 & 38.611 & -0.0317 & 0.095000 & -0.18700 \\
\hline-6414.815 & 46.125 & 56.011 & 38.498 & -0.0321 & 0.094000 & -0.19000 \\
\hline-6410.807 & 46.125 & 55.861 & 38.416 & -0.0326 & 0.093000 & -0.19100 \\
\hline-6406.799 & 45.875 & 55.755 & 38.342 & -0.0330 & 0.092000 & -0.19300 \\
\hline-6402.791 & 45.875 & 55.615 & 38.258 & -0.0335 & 0.091000 & -0.19400 \\
\hline-6398.783 & 45.625 & 55.436 & 38.193 & -0.0341 & 0.090000 & -0.19700 \\
\hline-6394.774 & 45.375 & 55.287 & 38.149 & -0.0346 & 0.088000 & -0.19900 \\
\hline-6390.766 & 45.375 & 55.120 & 38.058 & -0.0352 & 0.087000 & -0.20200 \\
\hline-6386.758 & 45.125 & 54.936 & 38.020 & -0.0357 & 0.086000 & -0.20200 \\
\hline-6382.750 & 45.125 & 54.724 & 37.976 & -0.0363 & 0.084000 & -0.20400 \\
\hline-6378.742 & 44.875 & 54.506 & 37.915 & -0.0370 & 0.083000 & -0.20400 \\
\hline-6374.734 & 44.875 & 54.292 & 37.885 & -0.0376 & 0.082000 & -0.20500 \\
\hline-6370.726 & 44.625 & 54.114 & 37.873 & -0.0382 & 0.081000 & -0.20700 \\
\hline-6366.718 & 44.625 & 53.854 & 37.905 & -0.0388 & 0.080000 & -0.20800 \\
\hline
\end{tabular}




\begin{tabular}{|c|c|c|c|c|c|c|}
\hline-6362.710 & 44.375 & 53.638 & 37.909 & -0.0392 & 0.078000 & -0.21100 \\
\hline-6358.701 & 44.125 & 53.401 & 37.914 & -0.0394 & 0.077000 & -0.21100 \\
\hline-6354.693 & 44.125 & 53.180 & 37.912 & -0.0394 & 0.075000 & -0.21100 \\
\hline-6350.685 & 43.875 & 52.943 & 37.908 & -0.0392 & 0.074000 & -0.21200 \\
\hline-6346.677 & 43.875 & 52.716 & 37.914 & -0.0388 & 0.073000 & -0.21100 \\
\hline-6342.669 & 43.625 & 52.489 & 37.937 & -0.0382 & 0.072000 & -0.20800 \\
\hline-6338.661 & 43.625 & 52.236 & 37.970 & -0.0374 & 0.070000 & -0.20700 \\
\hline-6334.653 & 43.375 & 51.958 & 38.029 & -0.0364 & 0.069000 & -0.20700 \\
\hline-6330.645 & 43.125 & 51.685 & 38.094 & -0.0353 & 0.065000 & -0.20600 \\
\hline-6326.636 & 43.125 & 51.415 & 38.167 & -0.0339 & 0.064000 & -0.20400 \\
\hline-6322.628 & 42.875 & 51.091 & 38.211 & -0.0324 & 0.061000 & -0.20200 \\
\hline-6318.620 & 42.875 & 50.780 & 38.297 & -0.0306 & 0.059000 & -0.19900 \\
\hline-6314.612 & 42.625 & 50.464 & 38.402 & -0.0287 & 0.057000 & -0.19300 \\
\hline-6310.604 & 42.625 & 50.169 & 38.466 & -0.0265 & 0.055000 & -0.18900 \\
\hline-6306.596 & 42.375 & 49.867 & 38.559 & -0.0242 & 0.052000 & -0.18300 \\
\hline-6302.588 & 42.375 & 49.581 & 38.617 & -0.0219 & 0.050000 & -0.17700 \\
\hline-6298.580 & 42.125 & 49.237 & 38.692 & -0.0197 & 0.047000 & -0.16900 \\
\hline-6294.572 & 42.125 & 48.860 & 38.807 & -0.0176 & 0.044000 & -0.16100 \\
\hline-6290.563 & 42.125 & 48.507 & 38.891 & -0.0157 & 0.042000 & -0.15200 \\
\hline-6286.555 & 41.875 & 48.175 & 38.958 & -0.0139 & 0.040000 & -0.14400 \\
\hline-6282.547 & 41.875 & 47.845 & 39.008 & -0.0122 & 0.038000 & -0.13500 \\
\hline-6278.539 & 41.875 & 47.535 & 39.070 & -0.0107 & 0.036000 & -0.12700 \\
\hline-6274.531 & 41.875 & 47.256 & 39.119 & -0.00930 & 0.034000 & -0.12000 \\
\hline-6270.523 & 41.875 & 46.950 & 39.170 & -0.00810 & 0.032000 & -0.11300 \\
\hline-6266.515 & 41.625 & 46.669 & 39.234 & -0.00700 & 0.031000 & -0.10600 \\
\hline-6262.507 & 41.625 & 46.434 & 39.287 & -0.00610 & 0.029000 & -0.10000 \\
\hline-6258.498 & 41.625 & 46.205 & 39.325 & -0.00520 & 0.027000 & -0.095000 \\
\hline-6254.490 & 41.625 & 46.001 & 39.363 & -0.00460 & 0.026000 & -0.089000 \\
\hline-6250.482 & 41.625 & 45.795 & 39.398 & -0.00410 & 0.025000 & -0.083000 \\
\hline-6246.474 & 41.625 & 45.608 & 39.442 & -0.00370 & 0.024000 & -0.078000 \\
\hline-6242.466 & 41.625 & 45.442 & 39.484 & -0.00330 & 0.023000 & -0.073000 \\
\hline-6238.458 & 41.625 & 45.268 & 39.515 & -0.00300 & 0.022000 & -0.069000 \\
\hline-6234.450 & 41.625 & 45.118 & 39.551 & -0.00270 & 0.021000 & -0.065000 \\
\hline-6230.442 & 41.625 & 44.959 & 39.584 & -0.00240 & 0.020000 & -0.061000 \\
\hline-6226.434 & 41.625 & 44.819 & 39.613 & -0.00210 & 0.020000 & -0.057000 \\
\hline-6222.425 & 41.625 & 44.688 & 39.646 & -0.00180 & 0.019000 & -0.053000 \\
\hline-6218.417 & 41.625 & 44.565 & 39.683 & -0.00160 & 0.019000 & -0.050000 \\
\hline-6214.409 & 41.625 & 44.433 & 39.713 & -0.00130 & 0.018000 & -0.048000 \\
\hline-6210.401 & 41.625 & 44.316 & 39.746 & -0.00100 & 0.018000 & -0.045000 \\
\hline-6206.393 & 41.625 & 44.206 & 39.780 & -0.000800 & 0.017000 & -0.042000 \\
\hline-6202.385 & 41.625 & 44.105 & 39.812 & -0.000500 & 0.017000 & -0.040000 \\
\hline-6198.377 & 41.625 & 44.008 & 39.842 & -0.000300 & 0.016000 & -0.038000 \\
\hline-6194.369 & 41.625 & 43.913 & 39.874 & 0.00 & 0.016000 & -0.036000 \\
\hline-6190.361 & 41.625 & 43.826 & 39.905 & 0.000200 & 0.016000 & -0.034000 \\
\hline-6186.352 & 41.625 & 43.746 & 39.935 & 0.000400 & 0.015000 & -0.032000 \\
\hline-6182.344 & 41.625 & 43.672 & 39.967 & 0.000700 & 0.015000 & -0.031000 \\
\hline-6178.336 & 41.625 & 43.597 & 39.998 & 0.000900 & 0.015000 & -0.030000 \\
\hline-6174.328 & 41.625 & 43.531 & 40.030 & 0.00110 & 0.015000 & -0.028000 \\
\hline-6170.320 & 41.625 & 43.466 & 40.059 & 0.00130 & 0.014000 & -0.027000 \\
\hline-6166.312 & 41.625 & 43.406 & 40.089 & 0.00150 & 0.014000 & -0.027000 \\
\hline-6162.304 & 41.625 & 43.354 & 40.120 & 0.00170 & 0.014000 & -0.026000 \\
\hline-6158.296 & 41.625 & 43.310 & 40.151 & 0.00190 & 0.014000 & -0.025000 \\
\hline-6154.287 & 41.625 & 43.263 & 40.182 & 0.00210 & 0.013000 & -0.024000 \\
\hline-6150.279 & 41.625 & 43.225 & 40.214 & 0.00230 & 0.013000 & -0.024000 \\
\hline
\end{tabular}




\begin{tabular}{|c|c|c|c|c|c|c|}
\hline-6146.271 & 41.625 & 43.191 & 40.244 & 0.00240 & 0.013000 & -0.023000 \\
\hline-6142.263 & 41.625 & 43.159 & 40.272 & 0.00260 & 0.013000 & -0.022000 \\
\hline-6138.255 & 41.625 & 43.137 & 40.300 & 0.00280 & 0.013000 & -0.022000 \\
\hline-6134.247 & 41.875 & 43.117 & 40.331 & 0.00290 & 0.012000 & -0.021000 \\
\hline-6130.239 & 41.875 & 43.099 & 40.363 & 0.00310 & 0.012000 & -0.020000 \\
\hline-6126.231 & 41.875 & 43.084 & 40.395 & 0.00320 & 0.012000 & -0.020000 \\
\hline-6122.223 & 41.875 & 43.072 & 40.428 & 0.00340 & 0.012000 & -0.019000 \\
\hline-6118.214 & 41.875 & 43.061 & 40.461 & 0.00350 & 0.012000 & -0.018000 \\
\hline-6114.206 & 41.875 & 43.052 & 40.491 & 0.00360 & 0.012000 & -0.018000 \\
\hline-6110.198 & 41.875 & 43.045 & 40.522 & 0.00370 & 0.011000 & -0.017000 \\
\hline-6106.190 & 41.875 & 43.040 & 40.552 & 0.00380 & 0.011000 & -0.016000 \\
\hline-6102.182 & 41.875 & 43.034 & 40.585 & 0.00400 & 0.011000 & -0.015000 \\
\hline-6098.174 & 41.875 & 43.032 & 40.616 & 0.00410 & 0.011000 & -0.014000 \\
\hline-6094.166 & 41.875 & 43.028 & 40.648 & 0.00420 & 0.011000 & -0.013000 \\
\hline-6090.158 & 41.875 & 43.027 & 40.681 & 0.00420 & 0.011000 & -0.011000 \\
\hline-6086.149 & 41.875 & 43.024 & 40.712 & 0.00430 & 0.011000 & -0.0100000 \\
\hline-6082.141 & 41.875 & 43.024 & 40.744 & 0.00440 & 0.011000 & -0.0090000 \\
\hline-6078.133 & 41.875 & 43.024 & 40.775 & 0.00450 & 0.011000 & -0.0080000 \\
\hline-6074.125 & 41.875 & 43.025 & 40.806 & 0.00450 & 0.011000 & -0.0070000 \\
\hline-6070.117 & 41.875 & 43.027 & 40.838 & 0.00460 & 0.0100000 & -0.0070000 \\
\hline-6066.109 & 42.125 & 43.027 & 40.871 & 0.00470 & 0.0100000 & -0.0060000 \\
\hline-6062.101 & 42.125 & 43.030 & 40.903 & 0.00470 & 0.0100000 & -0.0060000 \\
\hline-6058.093 & 42.125 & 43.032 & 40.935 & 0.00480 & 0.0100000 & -0.0060000 \\
\hline-6054.085 & 42.125 & 43.034 & 40.969 & 0.00480 & 0.0100000 & -0.0050000 \\
\hline-6050.076 & 42.125 & 43.034 & 41.000 & 0.00480 & 0.0100000 & -0.0050000 \\
\hline-6046.068 & 42.125 & 43.037 & 41.030 & 0.00480 & 0.0100000 & -0.0050000 \\
\hline-6042.060 & 42.125 & 43.040 & 41.062 & 0.00490 & 0.0100000 & -0.0050000 \\
\hline-6038.052 & 42.125 & 43.045 & 41.095 & 0.00490 & 0.0100000 & -0.0040000 \\
\hline-6034.044 & 42.125 & 43.048 & 41.126 & 0.00490 & 0.0100000 & -0.0040000 \\
\hline-6030.036 & 42.125 & 43.052 & 41.158 & 0.00490 & 0.0100000 & -0.0040000 \\
\hline-6026.028 & 42.125 & 43.058 & 41.187 & 0.00490 & 0.0100000 & -0.0040000 \\
\hline-6022.020 & 42.125 & 43.061 & 41.217 & 0.00490 & 0.0100000 & -0.0040000 \\
\hline-6018.012 & 42.125 & 43.065 & 41.248 & 0.00490 & 0.0100000 & -0.0040000 \\
\hline-6014.003 & 42.125 & 43.069 & 41.277 & 0.00480 & 0.0100000 & -0.0040000 \\
\hline-6009.995 & 42.125 & 43.075 & 41.305 & 0.00480 & 0.0100000 & -0.0040000 \\
\hline-6005.987 & 42.375 & 43.079 & 41.334 & 0.00480 & 0.0100000 & -0.0040000 \\
\hline-6001.979 & 42.375 & 43.085 & 41.361 & 0.00470 & 0.0090000 & -0.0040000 \\
\hline-5997.971 & 42.375 & 43.091 & 41.389 & 0.00470 & 0.0090000 & -0.0040000 \\
\hline-5993.963 & 42.375 & 43.096 & 41.416 & 0.00470 & 0.0090000 & -0.0040000 \\
\hline-5989.955 & 42.375 & 43.100 & 41.443 & 0.00460 & 0.0090000 & -0.0040000 \\
\hline-5985.947 & 42.375 & 43.105 & 41.469 & 0.00460 & 0.0090000 & -0.0040000 \\
\hline-5981.938 & 42.375 & 43.112 & 41.495 & 0.00450 & 0.0090000 & -0.0040000 \\
\hline-5977.930 & 42.375 & 43.117 & 41.521 & 0.00450 & 0.0090000 & -0.0040000 \\
\hline-5973.922 & 42.375 & 43.122 & 41.545 & 0.00440 & 0.0090000 & -0.0040000 \\
\hline-5969.914 & 42.375 & 43.129 & 41.569 & 0.00440 & 0.0090000 & -0.0040000 \\
\hline-5965.906 & 42.375 & 43.135 & 41.591 & 0.00440 & 0.0090000 & -0.0040000 \\
\hline-5961.898 & 42.375 & 43.142 & 41.612 & 0.00430 & 0.0090000 & -0.0040000 \\
\hline-5957.890 & 42.375 & 43.148 & 41.633 & 0.00430 & 0.0090000 & -0.0040000 \\
\hline-5953.882 & 42.375 & 43.155 & 41.653 & 0.00430 & 0.0090000 & -0.0040000 \\
\hline-5949.874 & 42.375 & 43.161 & 41.672 & 0.00420 & 0.0090000 & -0.0040000 \\
\hline-5945.865 & 42.625 & 43.168 & 41.692 & 0.00420 & 0.0090000 & -0.0030000 \\
\hline-5941.857 & 42.625 & 43.174 & 41.710 & 0.00420 & 0.0090000 & -0.0030000 \\
\hline-5937.849 & 42.625 & 43.180 & 41.729 & 0.00410 & 0.0090000 & -0.0030000 \\
\hline-5933.841 & 42.625 & 43.187 & 41.746 & 0.00410 & 0.0090000 & -0.0030000 \\
\hline
\end{tabular}




\begin{tabular}{|c|c|c|c|c|c|c|}
\hline-5929.833 & 42.625 & 43.195 & 41.760 & 0.00410 & 0.0090000 & -0.0030000 \\
\hline-5925.825 & 42.625 & 43.202 & 41.774 & 0.00410 & 0.0090000 & -0.0030000 \\
\hline-5921.817 & 42.625 & 43.209 & 41.786 & 0.00400 & 0.0090000 & -0.0030000 \\
\hline-5917.809 & 42.625 & 43.217 & 41.796 & 0.00400 & 0.0090000 & -0.0030000 \\
\hline-5913.801 & 42.625 & 43.225 & 41.806 & 0.00400 & 0.0090000 & -0.0030000 \\
\hline-5909.792 & 42.625 & 43.233 & 41.817 & 0.00400 & 0.0090000 & -0.0030000 \\
\hline-5905.784 & 42.625 & 43.242 & 41.826 & 0.00400 & 0.0090000 & -0.0030000 \\
\hline-5901.776 & 42.625 & 43.251 & 41.836 & 0.00400 & 0.0090000 & -0.0030000 \\
\hline-5897.768 & 42.625 & 43.260 & 41.840 & 0.00390 & 0.0090000 & -0.0030000 \\
\hline-5893.760 & 42.625 & 43.269 & 41.847 & 0.00390 & 0.0090000 & -0.0030000 \\
\hline-5889.752 & 42.625 & 43.279 & 41.855 & 0.00390 & 0.0090000 & -0.0030000 \\
\hline-5885.744 & 42.875 & 43.289 & 41.854 & 0.00390 & 0.0090000 & -0.0030000 \\
\hline-5881.736 & 42.875 & 43.300 & 41.857 & 0.00390 & 0.0100000 & -0.0030000 \\
\hline-5877.727 & 42.875 & 43.310 & 41.856 & 0.00390 & 0.0100000 & -0.0030000 \\
\hline-5873.719 & 42.875 & 43.322 & 41.857 & 0.00390 & 0.0100000 & -0.0030000 \\
\hline-5869.711 & 42.875 & 43.333 & 41.858 & 0.00390 & 0.0100000 & -0.0020000 \\
\hline-5865.703 & 42.875 & 43.345 & 41.858 & 0.00390 & 0.0100000 & -0.0020000 \\
\hline-5861.695 & 42.875 & 43.358 & 41.860 & 0.00390 & 0.0100000 & -0.0020000 \\
\hline-5857.687 & 42.875 & 43.370 & 41.864 & 0.00390 & 0.0100000 & -0.0020000 \\
\hline-5853.679 & 42.875 & 43.383 & 41.871 & 0.00390 & 0.0100000 & -0.0020000 \\
\hline-5849.671 & 42.875 & 43.397 & 41.880 & 0.00390 & 0.0100000 & -0.0020000 \\
\hline-5845.663 & 42.875 & 43.411 & 41.887 & 0.00390 & 0.011000 & -0.0020000 \\
\hline-5841.654 & 42.875 & 43.427 & 41.901 & 0.00390 & 0.011000 & -0.0010000 \\
\hline-5837.646 & 42.875 & 43.442 & 41.918 & 0.00390 & 0.011000 & -0.0010000 \\
\hline-5833.638 & 42.875 & 43.457 & 41.936 & 0.00390 & 0.011000 & -0.0010000 \\
\hline-5829.630 & 43.125 & 43.474 & 41.964 & 0.00400 & 0.012000 & -0.0010000 \\
\hline-5825.622 & 43.125 & 43.490 & 41.992 & 0.00400 & 0.012000 & -0.0010000 \\
\hline-5821.614 & 43.125 & 43.508 & 42.025 & 0.00400 & 0.013000 & -0.0010000 \\
\hline-5817.606 & 43.125 & 43.526 & 42.066 & 0.00400 & 0.013000 & 0.0000 \\
\hline-5813.598 & 43.125 & 43.544 & 42.113 & 0.00400 & 0.013000 & 0.0000 \\
\hline-5809.589 & 43.125 & 43.562 & 42.165 & 0.00400 & 0.014000 & 0.0000 \\
\hline-5805.581 & 43.125 & 43.581 & 42.227 & 0.00400 & 0.015000 & 0.0000 \\
\hline-5801.573 & 43.125 & 43.601 & 42.285 & 0.00410 & 0.015000 & 0.0000 \\
\hline-5797.565 & 43.125 & 43.623 & 42.348 & 0.00410 & 0.016000 & 0.0000 \\
\hline-5793.557 & 43.125 & 43.646 & 42.408 & 0.00410 & 0.016000 & 0.0000 \\
\hline-5789.549 & 43.125 & 43.673 & 42.461 & 0.00410 & 0.017000 & 0.0000 \\
\hline-5785.541 & 43.125 & 43.702 & 42.509 & 0.00420 & 0.017000 & 0.0000 \\
\hline-5781.533 & 43.125 & 43.730 & 42.555 & 0.00420 & 0.018000 & 0.0000 \\
\hline-5777.525 & 43.125 & 43.758 & 42.600 & 0.00420 & 0.018000 & 0.0000 \\
\hline-5773.516 & 43.375 & 43.787 & 42.644 & 0.00430 & 0.018000 & 0.0000 \\
\hline-5769.508 & 43.375 & 43.817 & 42.681 & 0.00430 & 0.019000 & 0.0000 \\
\hline-5765.500 & 43.375 & 43.847 & 42.717 & 0.00430 & 0.019000 & -0.0010000 \\
\hline-5761.492 & 43.375 & 43.879 & 42.748 & 0.00440 & 0.019000 & -0.0010000 \\
\hline-5757.484 & 43.375 & 43.911 & 42.778 & 0.00440 & 0.019000 & -0.0010000 \\
\hline-5753.476 & 43.375 & 43.945 & 42.803 & 0.00440 & 0.019000 & -0.0020000 \\
\hline-5749.468 & 43.375 & 43.978 & 42.826 & 0.00450 & 0.020000 & -0.0020000 \\
\hline-5745.460 & 43.375 & 44.015 & 42.850 & 0.00450 & 0.020000 & -0.0030000 \\
\hline-5741.452 & 43.375 & 44.054 & 42.869 & 0.00460 & 0.020000 & -0.0040000 \\
\hline-5737.443 & 43.375 & 44.092 & 42.888 & 0.00460 & 0.020000 & -0.0060000 \\
\hline-5733.435 & 43.375 & 44.131 & 42.904 & 0.00470 & 0.020000 & -0.0080000 \\
\hline-5729.427 & 43.375 & 44.173 & 42.915 & 0.00470 & 0.020000 & -0.011000 \\
\hline-5725.419 & 43.375 & 44.212 & 42.923 & 0.00480 & 0.020000 & -0.016000 \\
\hline-5721.411 & 43.375 & 44.252 & 42.925 & 0.00490 & 0.020000 & -0.021000 \\
\hline-5717.403 & 43.375 & 44.295 & 42.916 & 0.00500 & 0.020000 & -0.030000 \\
\hline
\end{tabular}




\begin{tabular}{|c|c|c|c|c|c|c|}
\hline-5713.395 & 43.625 & 44.332 & 42.896 & 0.00510 & 0.020000 & -0.045000 \\
\hline-5709.387 & 43.625 & 44.367 & 42.849 & 0.00520 & 0.019000 & -0.063000 \\
\hline-5705.378 & 43.625 & 44.405 & 42.750 & 0.00530 & 0.019000 & -0.076000 \\
\hline-5701.370 & 43.625 & 44.442 & 42.573 & 0.00540 & 0.019000 & -0.088000 \\
\hline-5697.362 & 43.625 & 44.470 & 42.307 & 0.00550 & 0.018000 & -0.098000 \\
\hline-5693.354 & 43.625 & 44.503 & 42.012 & 0.00560 & 0.018000 & -0.10900 \\
\hline-5689.346 & 43.625 & 44.530 & 41.661 & 0.00580 & 0.018000 & -0.12100 \\
\hline-5685.338 & 43.625 & 44.556 & 41.269 & 0.00590 & 0.017000 & -0.13300 \\
\hline-5681.330 & 43.625 & 44.576 & 40.850 & 0.00600 & 0.017000 & -0.14700 \\
\hline-5677.322 & 43.625 & 44.588 & 40.386 & 0.00570 & 0.016000 & -0.16100 \\
\hline-5673.314 & 43.625 & 44.606 & 39.893 & 0.00470 & 0.016000 & -0.17700 \\
\hline-5669.305 & 43.625 & 44.613 & 39.368 & 0.00290 & 0.016000 & -0.19500 \\
\hline-5665.297 & 43.625 & 44.625 & 38.796 & 0.000300 & 0.016000 & -0.21500 \\
\hline-5661.289 & 43.625 & 44.638 & 38.190 & -0.00310 & 0.015000 & -0.23900 \\
\hline-5657.281 & 43.625 & 44.653 & 37.508 & -0.00720 & 0.015000 & -0.26400 \\
\hline-5653.273 & 43.625 & 44.654 & 36.800 & -0.0121 & 0.014000 & -0.29600 \\
\hline-5649.265 & 43.625 & 44.654 & 35.983 & -0.0183 & 0.014000 & -0.33300 \\
\hline-5645.257 & 43.625 & 44.654 & 35.105 & -0.0280 & 0.014000 & -0.37500 \\
\hline-5641.249 & 43.375 & 44.655 & 34.093 & -0.0434 & 0.015000 & -0.42300 \\
\hline-5637.240 & 43.375 & 44.655 & 32.963 & -0.0650 & 0.016000 & -0.47100 \\
\hline-5633.232 & 43.375 & 44.646 & 31.747 & -0.0929 & 0.019000 & -0.53500 \\
\hline-5629.224 & 42.875 & 44.633 & 30.593 & -0.127 & 0.029000 & -0.59600 \\
\hline-5625.216 & 41.875 & 44.616 & 29.413 & -0.167 & 0.092000 & -0.66900 \\
\hline-5621.208 & 40.875 & 44.586 & 28.317 & -0.212 & 0.16600 & -0.74800 \\
\hline-5617.200 & 39.875 & 44.550 & 27.382 & -0.251 & 0.22200 & -0.83100 \\
\hline-5613.192 & 38.875 & 44.513 & 26.650 & -0.276 & 0.28400 & -0.90800 \\
\hline-5609.184 & 37.875 & 44.459 & 26.042 & -0.284 & 0.34800 & -0.98900 \\
\hline-5605.176 & 36.625 & 44.394 & 25.674 & -0.275 & 0.43600 & -1.0710 \\
\hline-5601.167 & 35.625 & 44.311 & 25.553 & -0.249 & 0.56800 & -1.1350 \\
\hline-5597.159 & 34.875 & 44.232 & 25.517 & -0.206 & 0.77800 & -1.1170 \\
\hline-5593.151 & 34.375 & 44.193 & 25.418 & -0.147 & 1.0600 & -1.0670 \\
\hline-5589.143 & 33.875 & 44.308 & 25.272 & -0.0720 & 1.3100 & -1.0010 \\
\hline-5585.135 & 33.875 & 45.022 & 25.193 & 0.0116 & 1.5140 & -0.93900 \\
\hline-5581.127 & 33.875 & 46.071 & 25.150 & 0.0974 & 1.6930 & -0.87600 \\
\hline-5577.119 & 34.375 & 46.934 & 25.137 & 0.184 & 1.7880 & -0.81000 \\
\hline-5573.111 & 34.875 & 47.609 & 25.187 & 0.268 & 1.8620 & -0.73500 \\
\hline-5569.103 & 35.625 & 48.098 & 25.289 & 0.332 & 1.8870 & -0.66500 \\
\hline-5565.094 & 37.125 & 48.491 & 25.454 & 0.359 & 1.8930 & -0.59600 \\
\hline-5561.086 & 39.375 & 48.811 & 25.655 & 0.345 & 1.9090 & -0.51800 \\
\hline-5557.078 & 41.375 & 49.124 & 25.962 & 0.295 & 1.8550 & -0.44800 \\
\hline-5553.070 & 42.375 & 49.358 & 26.237 & 0.228 & 1.7860 & -0.39100 \\
\hline-5549.062 & 42.875 & 49.579 & 26.476 & 0.165 & 1.6660 & -0.34600 \\
\hline-5545.054 & 43.125 & 49.698 & 26.811 & 0.109 & 1.4980 & -0.31100 \\
\hline-5541.046 & 43.375 & 49.745 & 27.313 & 0.0614 & 1.3030 & -0.29000 \\
\hline-5537.038 & 43.375 & 49.676 & 28.079 & 0.0208 & 1.1470 & -0.27600 \\
\hline-5533.029 & 43.125 & 49.508 & 29.303 & -0.0122 & 0.99100 & -0.26900 \\
\hline-5529.021 & 43.125 & 49.299 & 31.225 & -0.0376 & 0.84900 & -0.26200 \\
\hline-5525.013 & 42.875 & 49.044 & 33.734 & -0.0559 & 0.69900 & -0.25900 \\
\hline-5521.005 & 42.625 & 48.744 & 35.578 & -0.0693 & 0.57000 & -0.25600 \\
\hline-5516.997 & 42.375 & 48.419 & 36.303 & -0.0798 & 0.45800 & -0.25100 \\
\hline-5512.989 & 42.125 & 48.066 & 36.444 & -0.0880 & 0.37100 & -0.24700 \\
\hline-5508.981 & 41.875 & 47.722 & 36.342 & -0.0939 & 0.29100 & -0.24200 \\
\hline-5504.973 & 41.625 & 47.356 & 36.060 & -0.0974 & 0.22500 & -0.23700 \\
\hline-5500.965 & 41.375 & 46.924 & 35.734 & -0.0985 & 0.16500 & -0.23300 \\
\hline
\end{tabular}




\begin{tabular}{|c|c|c|c|c|c|c|}
\hline-5496.956 & 40.875 & 46.536 & 35.364 & -0.0975 & 0.069000 & -0.22800 \\
\hline-5492.948 & 40.625 & 46.136 & 34.964 & -0.0953 & -0.0100000 & -0.22400 \\
\hline-5488.940 & 40.375 & 45.716 & 34.585 & -0.0930 & -0.012000 & -0.21900 \\
\hline-5484.932 & 39.875 & 45.294 & 34.265 & -0.0906 & -0.012000 & -0.21500 \\
\hline-5480.924 & 39.625 & 44.877 & 33.987 & -0.0883 & -0.012000 & -0.21000 \\
\hline-5476.916 & 39.125 & 44.442 & 33.742 & -0.0860 & -0.011000 & -0.20500 \\
\hline-5472.908 & 38.875 & 44.009 & 33.530 & -0.0837 & -0.011000 & -0.20000 \\
\hline-5468.900 & 38.625 & 43.555 & 33.343 & -0.0814 & -0.0100000 & -0.19500 \\
\hline-5464.891 & 38.125 & 43.115 & 33.183 & -0.0792 & -0.0100000 & -0.19000 \\
\hline-5460.883 & 37.875 & 42.684 & 33.034 & -0.0769 & -0.0090000 & -0.18500 \\
\hline-5456.875 & 37.625 & 42.273 & 32.899 & -0.0747 & -0.0090000 & -0.18100 \\
\hline-5452.867 & 37.125 & 41.872 & 32.779 & -0.0725 & -0.0080000 & -0.17600 \\
\hline-5448.859 & 36.875 & 41.478 & 32.670 & -0.0704 & -0.0080000 & -0.17100 \\
\hline-5444.851 & 36.625 & 41.092 & 32.570 & -0.0682 & -0.0070000 & -0.16600 \\
\hline-5440.843 & 36.125 & 40.722 & 32.476 & -0.0661 & -0.0070000 & -0.16100 \\
\hline-5436.835 & 35.875 & 40.361 & 32.393 & -0.0640 & -0.0070000 & -0.15600 \\
\hline-5432.827 & 35.625 & 40.015 & 32.311 & -0.0619 & -0.0060000 & -0.15100 \\
\hline-5428.818 & 35.375 & 39.687 & 32.231 & -0.0598 & -0.0060000 & -0.14600 \\
\hline-5424.810 & 34.875 & 39.368 & 32.159 & -0.0577 & -0.0060000 & -0.14100 \\
\hline-5420.802 & 34.625 & 39.052 & 32.090 & -0.0557 & -0.0050000 & -0.13600 \\
\hline-5416.794 & 34.375 & 38.752 & 32.023 & -0.0537 & -0.0050000 & -0.13200 \\
\hline-5412.786 & 34.125 & 38.464 & 31.964 & -0.0517 & -0.0050000 & -0.12700 \\
\hline-5408.778 & 33.875 & 38.182 & 31.908 & -0.0497 & -0.0050000 & -0.12200 \\
\hline-5404.770 & 33.875 & 37.908 & 31.852 & -0.0478 & -0.0040000 & -0.11700 \\
\hline-5400.762 & 33.625 & 37.635 & 31.794 & -0.0458 & -0.0040000 & -0.11300 \\
\hline-5396.754 & 33.375 & 37.362 & 31.744 & -0.0439 & -0.0040000 & -0.10900 \\
\hline-5392.745 & 33.375 & 37.099 & 31.690 & -0.0420 & -0.0040000 & -0.10500 \\
\hline-5388.737 & 33.125 & 36.841 & 31.639 & -0.0402 & -0.0040000 & -0.10100 \\
\hline-5384.729 & 33.125 & 36.587 & 31.589 & -0.0383 & -0.0030000 & -0.097000 \\
\hline-5380.721 & 32.875 & 36.338 & 31.539 & -0.0365 & -0.0030000 & -0.094000 \\
\hline-5376.713 & 32.875 & 36.095 & 31.487 & -0.0346 & -0.0030000 & -0.090000 \\
\hline-5372.705 & 32.875 & 35.857 & 31.438 & -0.0329 & -0.0030000 & -0.087000 \\
\hline-5368.697 & 32.625 & 35.620 & 31.389 & -0.0311 & -0.0030000 & -0.084000 \\
\hline-5364.689 & 32.625 & 35.389 & 31.339 & -0.0294 & -0.0020000 & -0.081000 \\
\hline-5360.680 & 32.625 & 35.158 & 31.289 & -0.0278 & -0.0020000 & -0.078000 \\
\hline-5356.672 & 32.375 & 34.929 & 31.241 & -0.0262 & -0.0020000 & -0.076000 \\
\hline-5352.664 & 32.375 & 34.707 & 31.187 & -0.0247 & -0.0020000 & -0.074000 \\
\hline-5348.656 & 32.375 & 34.486 & 31.131 & -0.0233 & -0.0020000 & -0.072000 \\
\hline-5344.648 & 32.375 & 34.267 & 31.074 & -0.0219 & -0.0010000 & -0.070000 \\
\hline-5340.640 & 32.125 & 34.049 & 31.017 & -0.0206 & -0.0010000 & -0.068000 \\
\hline-5336.632 & 32.125 & 33.841 & 30.949 & -0.0193 & -0.0010000 & -0.067000 \\
\hline-5332.624 & 32.125 & 33.637 & 30.876 & -0.0181 & -0.0010000 & -0.065000 \\
\hline-5328.616 & 32.125 & 33.438 & 30.800 & -0.0170 & -0.0010000 & -0.065000 \\
\hline-5324.607 & 31.875 & 33.249 & 30.714 & -0.0159 & 0.0000 & -0.064000 \\
\hline-5320.599 & 31.875 & 33.080 & 30.627 & -0.0149 & 0.0000 & -0.063000 \\
\hline-5316.591 & 31.875 & 32.922 & 30.529 & -0.0140 & 0.0000 & -0.062000 \\
\hline-5312.583 & 31.875 & 32.787 & 30.422 & -0.0131 & 0.0010000 & -0.061000 \\
\hline-5308.575 & 31.625 & 32.671 & 30.306 & -0.0123 & 0.0010000 & -0.061000 \\
\hline-5304.567 & 31.625 & 32.571 & 30.187 & -0.0116 & 0.0010000 & -0.060000 \\
\hline-5300.559 & 31.625 & 32.487 & 30.051 & -0.0109 & 0.0020000 & -0.060000 \\
\hline-5296.551 & 31.625 & 32.413 & 29.921 & -0.0102 & 0.0020000 & -0.059000 \\
\hline-5292.542 & 31.375 & 32.350 & 29.780 & -0.00970 & 0.0030000 & -0.059000 \\
\hline-5288.534 & 31.375 & 32.299 & 29.635 & -0.00920 & 0.0030000 & -0.059000 \\
\hline-5284.526 & 31.375 & 32.251 & 29.479 & -0.00880 & 0.0040000 & -0.058000 \\
\hline
\end{tabular}




\begin{tabular}{|c|c|c|c|c|c|c|}
\hline-5280.518 & 31.375 & 32.208 & 29.332 & -0.00840 & 0.0050000 & -0.058000 \\
\hline-5276.510 & 31.375 & 32.169 & 29.192 & -0.00810 & 0.0060000 & -0.057000 \\
\hline-5272.502 & 31.125 & 32.131 & 29.044 & -0.00780 & 0.0070000 & -0.056000 \\
\hline-5268.494 & 31.125 & 32.097 & 28.907 & -0.00760 & 0.0090000 & -0.056000 \\
\hline-5264.486 & 31.125 & 32.063 & 28.779 & -0.00750 & 0.0100000 & -0.055000 \\
\hline-5260.478 & 31.125 & 32.031 & 28.657 & -0.00740 & 0.012000 & -0.055000 \\
\hline-5256.469 & 31.125 & 32.001 & 28.530 & -0.00750 & 0.013000 & -0.054000 \\
\hline-5252.461 & 30.875 & 31.972 & 28.408 & -0.00750 & 0.015000 & -0.053000 \\
\hline-5248.453 & 30.875 & 31.944 & 28.292 & -0.00760 & 0.017000 & -0.052000 \\
\hline-5244.445 & 30.875 & 31.918 & 28.180 & -0.00780 & 0.018000 & -0.051000 \\
\hline-5240.437 & 30.875 & 31.896 & 28.081 & -0.00790 & 0.020000 & -0.050000 \\
\hline-5236.429 & 30.875 & 31.872 & 27.981 & -0.00800 & 0.022000 & -0.049000 \\
\hline-5232.421 & 30.875 & 31.855 & 27.892 & -0.00820 & 0.024000 & -0.048000 \\
\hline-5228.413 & 30.625 & 31.838 & 27.808 & -0.00830 & 0.025000 & -0.047000 \\
\hline-5224.405 & 30.625 & 31.821 & 27.724 & -0.00830 & 0.027000 & -0.046000 \\
\hline-5220.396 & 30.625 & 31.807 & 27.660 & -0.00840 & 0.029000 & -0.045000 \\
\hline-5216.388 & 30.625 & 31.797 & 27.598 & -0.00850 & 0.031000 & -0.044000 \\
\hline-5212.380 & 30.625 & 31.789 & 27.545 & -0.00850 & 0.032000 & -0.042000 \\
\hline-5208.372 & 30.625 & 31.782 & 27.495 & -0.00850 & 0.034000 & -0.041000 \\
\hline-5204.364 & 30.375 & 31.779 & 27.451 & -0.00860 & 0.036000 & -0.039000 \\
\hline-5200.356 & 30.375 & 31.781 & 27.417 & -0.00860 & 0.038000 & -0.037000 \\
\hline-5196.348 & 30.375 & 31.788 & 27.397 & -0.00850 & 0.040000 & -0.036000 \\
\hline-5192.340 & 30.375 & 31.797 & 27.377 & -0.00850 & 0.042000 & -0.035000 \\
\hline-5188.331 & 30.375 & 31.813 & 27.367 & -0.00850 & 0.044000 & -0.033000 \\
\hline-5184.323 & 30.375 & 31.835 & 27.357 & -0.00840 & 0.046000 & -0.033000 \\
\hline-5180.315 & 30.375 & 31.870 & 27.360 & -0.00840 & 0.048000 & -0.032000 \\
\hline-5176.307 & 30.375 & 31.909 & 27.365 & -0.00830 & 0.050000 & -0.031000 \\
\hline-5172.299 & 30.125 & 31.953 & 27.364 & -0.00820 & 0.052000 & -0.030000 \\
\hline-5168.291 & 30.125 & 32.010 & 27.364 & -0.00810 & 0.054000 & -0.030000 \\
\hline-5164.283 & 30.125 & 32.072 & 27.378 & -0.00790 & 0.057000 & -0.029000 \\
\hline-5160.275 & 30.125 & 32.140 & 27.387 & -0.00780 & 0.059000 & -0.029000 \\
\hline-5156.267 & 30.125 & 32.215 & 27.398 & -0.00770 & 0.061000 & -0.028000 \\
\hline-5152.258 & 30.125 & 32.295 & 27.409 & -0.00750 & 0.064000 & -0.028000 \\
\hline-5148.250 & 30.125 & 32.387 & 27.420 & -0.00730 & 0.066000 & -0.028000 \\
\hline-5144.242 & 30.125 & 32.491 & 27.429 & -0.00710 & 0.069000 & -0.028000 \\
\hline-5140.234 & 30.125 & 32.605 & 27.449 & -0.00690 & 0.072000 & -0.028000 \\
\hline-5136.226 & 30.125 & 32.731 & 27.464 & -0.00670 & 0.076000 & -0.027000 \\
\hline-5132.218 & 30.125 & 32.871 & 27.472 & -0.00650 & 0.079000 & -0.028000 \\
\hline-5128.210 & 30.125 & 33.037 & 27.472 & -0.00620 & 0.083000 & -0.028000 \\
\hline-5124.202 & 30.125 & 33.207 & 27.480 & -0.00600 & 0.087000 & -0.028000 \\
\hline-5120.193 & 30.125 & 33.389 & 27.481 & -0.00570 & 0.090000 & -0.029000 \\
\hline-5116.185 & 29.875 & 33.587 & 27.477 & -0.00540 & 0.096000 & -0.029000 \\
\hline-5112.177 & 29.875 & 33.785 & 27.467 & -0.00510 & 0.10100 & -0.030000 \\
\hline-5108.169 & 29.875 & 34.029 & 27.462 & -0.00480 & 0.10600 & -0.031000 \\
\hline-5104.161 & 29.875 & 34.298 & 27.450 & -0.00440 & 0.11200 & -0.032000 \\
\hline-5100.153 & 29.875 & 34.587 & 27.444 & -0.00410 & 0.11900 & -0.033000 \\
\hline-5096.145 & 29.875 & 34.883 & 27.420 & -0.00370 & 0.12700 & -0.035000 \\
\hline-5092.137 & 29.875 & 35.229 & 27.403 & -0.00340 & 0.13400 & -0.037000 \\
\hline-5088.129 & 29.875 & 35.604 & 27.363 & -0.00300 & 0.14200 & -0.039000 \\
\hline-5084.120 & 29.875 & 36.019 & 27.334 & -0.00260 & 0.15100 & -0.041000 \\
\hline-5080.112 & 29.875 & 36.437 & 27.294 & -0.00220 & 0.16200 & -0.045000 \\
\hline-5076.104 & 29.875 & 36.954 & 27.254 & -0.00170 & 0.17500 & -0.048000 \\
\hline-5072.096 & 29.875 & 37.454 & 27.213 & -0.00130 & 0.19100 & -0.051000 \\
\hline-5068.088 & 29.875 & 38.007 & 27.168 & -0.000800 & 0.20800 & -0.055000 \\
\hline
\end{tabular}




$\begin{array}{lllllll}-5064.080 & 29.875 & 38.673 & 27.119 & -0.000400 & 0.22500 & -0.059000 \\ -5060.072 & 29.875 & 39.334 & 27.068 & 1.00 \mathrm{e}-04 & 0.24600 & -0.062000 \\ -5056.064 & 29.875 & 40.140 & 27.014 & 0.000600 & 0.27200 & -0.067000 \\ -5052.056 & 29.875 & 40.933 & 26.954 & 0.00110 & 0.29800 & -0.072000 \\ -5048.047 & 29.875 & 41.869 & 26.888 & 0.00160 & 0.32800 & -0.078000 \\ -5044.039 & 29.875 & 42.850 & 26.811 & 0.00220 & 0.35900 & -0.084000 \\ -5040.031 & 29.875 & 43.905 & 26.725 & 0.00270 & 0.39700 & -0.093000 \\ -5036.023 & 29.875 & 45.028 & 26.629 & 0.00330 & 0.43200 & -0.10000 \\ -5032.015 & 29.875 & 46.222 & 26.519 & 0.00390 & 0.48100 & -0.11000 \\ -5028.007 & 29.875 & 47.451 & 26.405 & 0.00450 & 0.54000 & -0.12100 \\ -5023.999 & 29.875 & 48.855 & 26.266 & 0.00510 & 0.60900 & -0.13400 \\ -5019.991 & 29.875 & 50.363 & 26.115 & 0.00570 & 0.69800 & -0.15000 \\ -5015.982 & 29.875 & 51.926 & 25.912 & 0.00630 & 0.80600 & -0.17100 \\ -5011.974 & 29.875 & 53.727 & 25.689 & 0.00700 & 0.95800 & -0.20100 \\ -5007.966 & 29.875 & 55.845 & 25.379 & 0.00760 & 1.1950 & -0.23500 \\ -5003.958 & 29.875 & 58.609 & 24.940 & 0.00830 & 1.5900 & -0.29100 \\ -4999.950 & 29.875 & 63.915 & 23.933 & 0.00900 & 2.4660 & -0.38100\end{array}$




\begin{tabular}{|c|c|c|c|c|c|c|}
\hline Date & Median (muT) & Cred Int Upper & Cred Int Lower & dFdt (muT/vr) & ner & Inwer \\
\hline-7000.000 & 55.125 & & & & & \\
\hline-6995.992 & 125 & 931 & 4 & 0151 & 14130 & - \\
\hline-6991.984 & 55.125 & 67.596 & 25.112 & 0.0145 & 1.1230 & -0.65200 \\
\hline-6987.976 & 55.125 & 67.341 & 25.904 & 0.0138 & 0.95600 & -0.56900 \\
\hline-6983.968 & 55.125 & 67.121 & 26.540 & 0.0131 & 0.83300 & -0.51300 \\
\hline-6979.959 & 55.375 & 66.937 & 27.084 & 0.0125 & 0.73600 & -0.46500 \\
\hline-6975.951 & 55.375 & 66.782 & 27.621 & 0.0118 & 0.67300 & -0.43000 \\
\hline-6971.943 & 55.375 & 66.625 & 28.076 & 0.0112 & 0.62100 & -0.39800 \\
\hline-6967.935 & 55.375 & 66.477 & 28.443 & 0.0106 & 0.57500 & -0.37300 \\
\hline-6963.927 & 55.375 & 66.330 & 28.794 & 0.00990 & 0.54100 & -0.35300 \\
\hline-6959.919 & 55.375 & 66.200 & 29.100 & 0.00930 & 0.50900 & -0.33600 \\
\hline-6955.911 & 55.625 & 66.061 & 29.336 & 0.00870 & 0.48500 & -0.32000 \\
\hline-6951.903 & 55.625 & 65.941 & 29.606 & 0.00800 & 0.46100 & -0.30700 \\
\hline-6947.894 & 55.625 & 65.815 & 29.858 & 0.00740 & 0.44100 & -0.29500 \\
\hline-6943.886 & 55.625 & 65.703 & 30.083 & 0.00680 & 0.42400 & -0.28400 \\
\hline-6939.878 & 55.625 & 65.580 & 30.293 & 0.00620 & 0.40700 & -0.27400 \\
\hline-6935.870 & 55.625 & 65.477 & 30.470 & 0.00560 & 0.39500 & -0.26500 \\
\hline-6931.862 & 55.625 & 65.347 & 30.713 & 0.00500 & 0.38000 & -0.25600 \\
\hline-6927.854 & 55.625 & 65.237 & 30.884 & 0.00440 & 0.36900 & -0.24700 \\
\hline-6923.846 & 55.625 & 65.136 & 31.117 & 0.00390 & 0.35800 & -0.24000 \\
\hline-6919.838 & 55.875 & 65.037 & 31.312 & 0.00330 & 0.34700 & -0.23500 \\
\hline-6915.830 & 55.875 & 64.924 & 31.478 & 0.00270 & 0.34000 & -0.22700 \\
\hline-6911.821 & 55.875 & 64.811 & 31.663 & 0.00220 & 0.33200 & -0.22200 \\
\hline-6907.813 & 55.875 & 64.696 & 31.828 & 0.00160 & 0.32400 & -0.21500 \\
\hline-6903.805 & 55.875 & 64.586 & 32.031 & 0.00100 & 0.31700 & -0.20900 \\
\hline-6899.797 & 55.875 & 64.477 & 32.201 & 0.000500 & 0.31100 & -0.20400 \\
\hline-6895.789 & 55.875 & 64.379 & 32.401 & $-1.00 e-04$ & 0.30600 & -0.19800 \\
\hline-6891.781 & 55.875 & 64.277 & 32.599 & -0.000600 & 0.30200 & -0.19200 \\
\hline-6887.773 & 55.875 & 64.175 & 32.817 & -0.00110 & 0.29700 & -0.18900 \\
\hline-6883.765 & 55.875 & 64.077 & 33.075 & -0.00170 & 0.29400 & -0.18500 \\
\hline-6879.757 & 55.875 & 63.971 & 33.346 & -0.00220 & 0.28800 & -0.18200 \\
\hline-6875.748 & 55.875 & 63.868 & 33.513 & -0.00270 & 0.28300 & -0.17700 \\
\hline-6871.740 & 55.875 & 63.775 & 33.814 & -0.00320 & 0.28000 & -0.17200 \\
\hline-6867.732 & 55.875 & 63.693 & 34.050 & -0.00370 & 0.27600 & -0.17000 \\
\hline-6863.724 & 55.625 & 63.606 & 34.291 & -0.00420 & 0.27300 & -0.16600 \\
\hline-6859.716 & 55.625 & 63.515 & 34.512 & -0.00470 & 0.27000 & -0.16300 \\
\hline-6855.708 & 55.625 & 63.417 & 34.767 & -0.00520 & 0.26600 & -0.15900 \\
\hline-6851.700 & 55.625 & 63.331 & 35.045 & -0.00570 & 0.26400 & -0.15500 \\
\hline-6847.692 & 55.625 & 63.251 & 35.310 & -0.00620 & 0.26000 & -0.15200 \\
\hline-6843.683 & 55.625 & 63.175 & 35.581 & -0.00660 & 0.25400 & -0.15000 \\
\hline-6839.675 & 55.625 & 63.072 & 35.852 & -0.00710 & 0.25000 & -0.14600 \\
\hline-6835.667 & 55.625 & 62.955 & 36.156 & -0.00760 & 0.24800 & -0.14200 \\
\hline-6831.659 & 55.625 & 62.861 & 36.433 & -0.00800 & 0.24400 & -0.13900 \\
\hline-6827.651 & 55.375 & 62.774 & 36.678 & -0.00850 & 0.24100 & -0.13600 \\
\hline-6823.643 & 55.375 & 62.673 & 37.001 & -0.00890 & 0.24000 & -0.13200 \\
\hline-6819.635 & 55.375 & 62.560 & 37.324 & -0.00940 & 0.23500 & -0.12900 \\
\hline-6815.627 & 55.375 & 62.462 & 37.761 & -0.00980 & 0.23200 & -0.12600 \\
\hline-6811.619 & 55.375 & 62.370 & 38.205 & -0.0103 & 0.22900 & -0.12300 \\
\hline-6807.610 & 55.375 & 62.247 & 38.606 & -0.0107 & 0.22700 & -0.12000 \\
\hline-6803.602 & 55.375 & 62.146 & 39.073 & -0.0111 & 0.22300 & -0.11800 \\
\hline-6799.594 & 55.125 & 62.014 & 39.526 & -0.0115 & 0.21900 & -0.11500 \\
\hline
\end{tabular}




\begin{tabular}{|c|c|c|c|c|c|c|}
\hline-6795.586 & 55.125 & 61.894 & 39.874 & -0.0119 & 0.21500 & -0.11300 \\
\hline-6791.578 & 55.125 & 61.777 & 40.306 & -0.0123 & 0.21200 & -0.11100 \\
\hline-6787.570 & 55.125 & 61.646 & 40.742 & -0.0127 & 0.20700 & -0.10900 \\
\hline-6783.562 & 55.125 & 61.495 & 41.235 & -0.0131 & 0.20100 & -0.10700 \\
\hline-6779.554 & 54.875 & 61.365 & 41.762 & -0.0135 & 0.19800 & -0.10500 \\
\hline-6775.545 & 54.875 & 61.226 & 42.230 & -0.0139 & 0.19500 & -0.10300 \\
\hline-6771.537 & 54.875 & 61.079 & 42.681 & -0.0143 & 0.19000 & -0.10200 \\
\hline-6767.529 & 54.875 & 60.941 & 43.111 & -0.0147 & 0.18500 & -0.10100 \\
\hline-6763.521 & 54.625 & 60.801 & 43.709 & -0.0150 & 0.18000 & -0.10000 \\
\hline-6759.513 & 54.625 & 60.636 & 44.190 & -0.0154 & 0.17500 & -0.099000 \\
\hline-6755.505 & 54.625 & 60.494 & 44.704 & -0.0158 & 0.16800 & -0.099000 \\
\hline-6751.497 & 54.625 & 60.321 & 45.203 & -0.0161 & 0.16200 & -0.098000 \\
\hline-6747.489 & 54.375 & 60.154 & 45.681 & -0.0165 & 0.15600 & -0.097000 \\
\hline-6743.481 & 54.375 & 59.981 & 46.144 & -0.0168 & 0.15100 & -0.096000 \\
\hline-6739.472 & 54.375 & 59.823 & 46.568 & -0.0172 & 0.14500 & -0.094000 \\
\hline-6735.464 & 54.375 & 59.633 & 47.065 & -0.0175 & 0.14000 & -0.094000 \\
\hline-6731.456 & 54.125 & 59.451 & 47.504 & -0.0178 & 0.13400 & -0.094000 \\
\hline-6727.448 & 54.125 & 59.282 & 47.927 & -0.0181 & 0.12700 & -0.094000 \\
\hline-6723.440 & 54.125 & 59.088 & 48.277 & -0.0185 & 0.12100 & -0.094000 \\
\hline-6719.432 & 53.875 & 58.912 & 48.514 & -0.0188 & 0.11500 & -0.094000 \\
\hline-6715.424 & 53.875 & 58.721 & 48.707 & -0.0191 & 0.10900 & -0.094000 \\
\hline-6711.416 & 53.875 & 58.562 & 48.841 & -0.0194 & 0.10400 & -0.094000 \\
\hline-6707.408 & 53.875 & 58.416 & 48.967 & -0.0197 & 0.099000 & -0.093000 \\
\hline-6703.399 & 53.625 & 58.261 & 49.054 & -0.0200 & 0.096000 & -0.093000 \\
\hline-6699.391 & 53.625 & 58.123 & 49.082 & -0.0202 & 0.091000 & -0.093000 \\
\hline-6695.383 & 53.625 & 57.996 & 49.099 & -0.0205 & 0.087000 & -0.093000 \\
\hline-6691.375 & 53.375 & 57.879 & 49.085 & -0.0208 & 0.082000 & -0.093000 \\
\hline-6687.367 & 53.375 & 57.749 & 49.054 & -0.0211 & 0.078000 & -0.093000 \\
\hline-6683.359 & 53.375 & 57.633 & 48.995 & -0.0213 & 0.075000 & -0.093000 \\
\hline-6679.351 & 53.125 & 57.529 & 48.939 & -0.0216 & 0.072000 & -0.093000 \\
\hline-6675.343 & 53.125 & 57.433 & 48.883 & -0.0218 & 0.070000 & -0.093000 \\
\hline-6671.334 & 52.875 & 57.351 & 48.799 & -0.0221 & 0.067000 & -0.093000 \\
\hline-6667.326 & 52.875 & 57.272 & 48.707 & -0.0223 & 0.065000 & -0.093000 \\
\hline-6663.318 & 52.875 & 57.198 & 48.605 & -0.0226 & 0.062000 & -0.093000 \\
\hline-6659.310 & 52.625 & 57.126 & 48.505 & -0.0228 & 0.061000 & -0.093000 \\
\hline-6655.302 & 52.625 & 57.089 & 48.384 & -0.0230 & 0.060000 & -0.093000 \\
\hline-6651.294 & 52.625 & 57.031 & 48.264 & -0.0232 & 0.059000 & -0.093000 \\
\hline-6647.286 & 52.375 & 57.002 & 48.139 & -0.0235 & 0.057000 & -0.092000 \\
\hline-6643.278 & 52.375 & 56.972 & 47.997 & -0.0237 & 0.056000 & -0.093000 \\
\hline-6639.270 & 52.375 & 56.950 & 47.846 & -0.0239 & 0.056000 & -0.093000 \\
\hline-6635.261 & 52.125 & 56.936 & 47.675 & -0.0241 & 0.055000 & -0.092000 \\
\hline-6631.253 & 52.125 & 56.932 & 47.507 & -0.0243 & 0.055000 & -0.093000 \\
\hline-6627.245 & 51.875 & 56.934 & 47.332 & -0.0245 & 0.055000 & -0.092000 \\
\hline-6623.237 & 51.875 & 56.941 & 47.141 & -0.0247 & 0.055000 & -0.092000 \\
\hline-6619.229 & 51.875 & 56.949 & 46.939 & -0.0248 & 0.055000 & -0.093000 \\
\hline-6615.221 & 51.625 & 56.970 & 46.747 & -0.0250 & 0.055000 & -0.093000 \\
\hline-6611.213 & 51.625 & 56.995 & 46.536 & -0.0252 & 0.056000 & -0.094000 \\
\hline-6607.205 & 51.375 & 57.011 & 46.319 & -0.0253 & 0.057000 & -0.094000 \\
\hline-6603.196 & 51.375 & 57.018 & 46.133 & -0.0255 & 0.058000 & -0.094000 \\
\hline-6599.188 & 51.375 & 57.044 & 45.930 & -0.0256 & 0.059000 & -0.095000 \\
\hline-6595.180 & 51.125 & 57.058 & 45.739 & -0.0258 & 0.060000 & -0.096000 \\
\hline-6591.172 & 51.125 & 57.073 & 45.541 & -0.0259 & 0.061000 & -0.096000 \\
\hline-6587.164 & 50.875 & 57.108 & 45.332 & -0.0261 & 0.062000 & -0.097000 \\
\hline-6583.156 & 50.875 & 57.132 & 45.104 & -0.0262 & 0.064000 & -0.098000 \\
\hline
\end{tabular}




\begin{tabular}{|c|c|c|c|c|c|c|}
\hline-6579.148 & 50.875 & 57.122 & 44.907 & -0.0263 & 0.065000 & -0.099000 \\
\hline-6575.140 & 50.625 & 57.130 & 44.695 & -0.0265 & 0.066000 & -0.10000 \\
\hline-6571.132 & 50.625 & 57.164 & 44.506 & -0.0266 & 0.067000 & -0.10100 \\
\hline-6567.123 & 50.375 & 57.186 & 44.288 & -0.0267 & 0.068000 & -0.10400 \\
\hline-6563.115 & 50.375 & 57.217 & 44.055 & -0.0268 & 0.070000 & -0.10500 \\
\hline-6559.107 & 50.125 & 57.210 & 43.860 & -0.0269 & 0.071000 & -0.10600 \\
\hline-6555.099 & 50.125 & 57.210 & 43.625 & -0.0270 & 0.073000 & -0.10800 \\
\hline-6551.091 & 50.125 & 57.226 & 43.394 & -0.0271 & 0.074000 & -0.10800 \\
\hline-6547.083 & 49.875 & 57.219 & 43.154 & -0.0272 & 0.075000 & -0.11000 \\
\hline-6543.075 & 49.875 & 57.234 & 42.909 & -0.0272 & 0.077000 & -0.11200 \\
\hline-6539.067 & 49.625 & 57.220 & 42.696 & -0.0273 & 0.078000 & -0.11400 \\
\hline-6535.059 & 49.625 & 57.224 & 42.450 & -0.0274 & 0.079000 & -0.11600 \\
\hline-6531.050 & 49.375 & 57.227 & 42.224 & -0.0274 & 0.081000 & -0.11800 \\
\hline-6527.042 & 49.375 & 57.231 & 41.979 & -0.0275 & 0.082000 & -0.12100 \\
\hline-6523.034 & 49.375 & 57.252 & 41.783 & -0.0275 & 0.083000 & -0.12300 \\
\hline-6519.026 & 49.125 & 57.215 & 41.570 & -0.0276 & 0.084000 & -0.12500 \\
\hline-6515.018 & 49.125 & 57.215 & 41.366 & -0.0276 & 0.086000 & -0.12600 \\
\hline-6511.010 & 48.875 & 57.195 & 41.188 & -0.0277 & 0.087000 & -0.12900 \\
\hline-6507.002 & 48.875 & 57.186 & 41.038 & -0.0277 & 0.089000 & -0.13100 \\
\hline-6502.994 & 48.625 & 57.147 & 40.873 & -0.0277 & 0.090000 & -0.13300 \\
\hline-6498.985 & 48.625 & 57.143 & 40.732 & -0.0278 & 0.090000 & -0.13500 \\
\hline-6494.977 & 48.375 & 57.131 & 40.609 & -0.0278 & 0.091000 & -0.13800 \\
\hline-6490.969 & 48.375 & 57.081 & 40.476 & -0.0278 & 0.093000 & -0.14000 \\
\hline-6486.961 & 48.375 & 57.057 & 40.359 & -0.0279 & 0.094000 & -0.14200 \\
\hline-6482.953 & 48.125 & 57.019 & 40.260 & -0.0280 & 0.094000 & -0.14500 \\
\hline-6478.945 & 48.125 & 57.009 & 40.157 & -0.0280 & 0.094000 & -0.14800 \\
\hline-6474.937 & 47.875 & 56.980 & 40.058 & -0.0282 & 0.094000 & -0.15000 \\
\hline-6470.929 & 47.875 & 56.935 & 39.960 & -0.0283 & 0.094000 & -0.15200 \\
\hline-6466.921 & 47.625 & 56.876 & 39.875 & -0.0284 & 0.095000 & -0.15300 \\
\hline-6462.912 & 47.625 & 56.798 & 39.782 & -0.0286 & 0.093000 & -0.15700 \\
\hline-6458.904 & 47.375 & 56.739 & 39.698 & -0.0287 & 0.093000 & -0.15800 \\
\hline-6454.896 & 47.375 & 56.691 & 39.599 & -0.0289 & 0.092000 & -0.16000 \\
\hline-6450.888 & 47.125 & 56.618 & 39.501 & -0.0291 & 0.092000 & -0.16200 \\
\hline-6446.880 & 47.125 & 56.517 & 39.398 & -0.0294 & 0.091000 & -0.16500 \\
\hline-6442.872 & 46.875 & 56.450 & 39.313 & -0.0296 & 0.090000 & -0.16800 \\
\hline-6438.864 & 46.875 & 56.371 & 39.202 & -0.0299 & 0.089000 & -0.17000 \\
\hline-6434.856 & 46.625 & 56.272 & 39.130 & -0.0301 & 0.090000 & -0.17100 \\
\hline-6430.847 & 46.625 & 56.207 & 39.066 & -0.0304 & 0.089000 & -0.17500 \\
\hline-6426.839 & 46.375 & 56.108 & 38.987 & -0.0307 & 0.088000 & -0.17600 \\
\hline-6422.831 & 46.375 & 56.024 & 38.900 & -0.0310 & 0.087000 & -0.17700 \\
\hline-6418.823 & 46.125 & 55.907 & 38.830 & -0.0314 & 0.086000 & -0.18100 \\
\hline-6414.815 & 46.125 & 55.786 & 38.774 & -0.0317 & 0.085000 & -0.18200 \\
\hline-6410.807 & 45.875 & 55.626 & 38.688 & -0.0321 & 0.084000 & -0.18500 \\
\hline-6406.799 & 45.875 & 55.486 & 38.616 & -0.0325 & 0.085000 & -0.18600 \\
\hline-6402.791 & 45.625 & 55.353 & 38.534 & -0.0329 & 0.085000 & -0.18800 \\
\hline-6398.783 & 45.625 & 55.181 & 38.473 & -0.0333 & 0.083000 & -0.18900 \\
\hline-6394.774 & 45.375 & 55.035 & 38.382 & -0.0337 & 0.081000 & -0.19300 \\
\hline-6390.766 & 45.375 & 54.855 & 38.346 & -0.0342 & 0.081000 & -0.19400 \\
\hline-6386.758 & 45.125 & 54.666 & 38.300 & -0.0346 & 0.080000 & -0.19400 \\
\hline-6382.750 & 45.125 & 54.475 & 38.218 & -0.0351 & 0.079000 & -0.19600 \\
\hline-6378.742 & 44.875 & 54.288 & 38.159 & -0.0356 & 0.078000 & -0.19800 \\
\hline-6374.734 & 44.875 & 54.082 & 38.112 & -0.0361 & 0.076000 & -0.20000 \\
\hline-6370.726 & 44.625 & 53.865 & 38.075 & -0.0366 & 0.076000 & -0.19900 \\
\hline-6366.718 & 44.625 & 53.652 & 38.017 & -0.0371 & 0.075000 & -0.20000 \\
\hline
\end{tabular}




\begin{tabular}{|c|c|c|c|c|c|c|}
\hline-6362.710 & 44.375 & 53.413 & 38.045 & -0.0374 & 0.074000 & -0.20000 \\
\hline-6358.701 & 44.125 & 53.231 & 38.042 & -0.0375 & 0.073000 & -0.20100 \\
\hline-6354.693 & 44.125 & 52.994 & 38.044 & -0.0375 & 0.070000 & -0.20200 \\
\hline-6350.685 & 43.875 & 52.764 & 38.056 & -0.0373 & 0.070000 & -0.20300 \\
\hline-6346.677 & 43.875 & 52.497 & 38.076 & -0.0370 & 0.069000 & -0.20200 \\
\hline-6342.669 & 43.625 & 52.226 & 38.089 & -0.0365 & 0.067000 & -0.20200 \\
\hline-6338.661 & 43.375 & 51.978 & 38.127 & -0.0358 & 0.065000 & -0.20300 \\
\hline-6334.653 & 43.375 & 51.694 & 38.160 & -0.0350 & 0.062000 & -0.20100 \\
\hline-6330.645 & 43.125 & 51.397 & 38.223 & -0.0340 & 0.059000 & -0.19900 \\
\hline-6326.636 & 43.125 & 51.096 & 38.264 & -0.0329 & 0.057000 & -0.19600 \\
\hline-6322.628 & 42.875 & 50.827 & 38.326 & -0.0316 & 0.054000 & -0.19500 \\
\hline-6318.620 & 42.875 & 50.512 & 38.391 & -0.0302 & 0.053000 & -0.19000 \\
\hline-6314.612 & 42.625 & 50.205 & 38.454 & -0.0286 & 0.051000 & -0.18800 \\
\hline-6310.604 & 42.625 & 49.865 & 38.540 & -0.0268 & 0.049000 & -0.18200 \\
\hline-6306.596 & 42.375 & 49.540 & 38.632 & -0.0249 & 0.047000 & -0.17700 \\
\hline-6302.588 & 42.375 & 49.214 & 38.710 & -0.0230 & 0.045000 & -0.16900 \\
\hline-6298.580 & 42.125 & 48.879 & 38.782 & -0.0211 & 0.044000 & -0.16200 \\
\hline-6294.572 & 42.125 & 48.548 & 38.864 & -0.0193 & 0.041000 & -0.15300 \\
\hline-6290.563 & 42.125 & 48.230 & 38.930 & -0.0176 & 0.038000 & -0.14500 \\
\hline-6286.555 & 41.875 & 47.904 & 39.010 & -0.0160 & 0.036000 & -0.13700 \\
\hline-6282.547 & 41.875 & 47.568 & 39.066 & -0.0145 & 0.033000 & -0.13000 \\
\hline-6278.539 & 41.875 & 47.243 & 39.116 & -0.0131 & 0.031000 & -0.12100 \\
\hline-6274.531 & 41.875 & 46.976 & 39.163 & -0.0117 & 0.030000 & -0.11400 \\
\hline-6270.523 & 41.875 & 46.693 & 39.207 & -0.0105 & 0.028000 & -0.10700 \\
\hline-6266.515 & 41.625 & 46.445 & 39.255 & -0.00930 & 0.027000 & -0.10100 \\
\hline-6262.507 & 41.625 & 46.217 & 39.296 & -0.00830 & 0.026000 & -0.095000 \\
\hline-6258.498 & 41.625 & 46.018 & 39.341 & -0.00730 & 0.025000 & -0.090000 \\
\hline-6254.490 & 41.625 & 45.820 & 39.383 & -0.00640 & 0.024000 & -0.084000 \\
\hline-6250.482 & 41.625 & 45.622 & 39.414 & -0.00560 & 0.022000 & -0.079000 \\
\hline-6246.474 & 41.625 & 45.451 & 39.447 & -0.00490 & 0.022000 & -0.074000 \\
\hline-6242.466 & 41.625 & 45.296 & 39.482 & -0.00420 & 0.021000 & -0.070000 \\
\hline-6238.458 & 41.625 & 45.138 & 39.510 & -0.00360 & 0.020000 & -0.065000 \\
\hline-6234.450 & 41.625 & 44.991 & 39.541 & -0.00300 & 0.019000 & -0.061000 \\
\hline-6230.442 & 41.625 & 44.847 & 39.575 & -0.00240 & 0.019000 & -0.057000 \\
\hline-6226.434 & 41.625 & 44.720 & 39.608 & -0.00180 & 0.018000 & -0.054000 \\
\hline-6222.425 & 41.625 & 44.582 & 39.635 & -0.00130 & 0.018000 & -0.051000 \\
\hline-6218.417 & 41.625 & 44.448 & 39.662 & -0.000800 & 0.018000 & -0.047000 \\
\hline-6214.409 & 41.625 & 44.336 & 39.691 & -0.000300 & 0.017000 & -0.045000 \\
\hline-6210.401 & 41.625 & 44.224 & 39.719 & $1.00 \mathrm{e}-04$ & 0.017000 & -0.042000 \\
\hline-6206.393 & 41.625 & 44.121 & 39.747 & 0.000600 & 0.017000 & -0.040000 \\
\hline-6202.385 & 41.625 & 44.022 & 39.778 & 0.00100 & 0.016000 & -0.038000 \\
\hline-6198.377 & 41.625 & 43.933 & 39.804 & 0.00140 & 0.016000 & -0.036000 \\
\hline-6194.369 & 41.625 & 43.850 & 39.835 & 0.00170 & 0.016000 & -0.034000 \\
\hline-6190.361 & 41.625 & 43.765 & 39.870 & 0.00210 & 0.016000 & -0.033000 \\
\hline-6186.352 & 41.625 & 43.679 & 39.900 & 0.00240 & 0.016000 & -0.031000 \\
\hline-6182.344 & 41.625 & 43.600 & 39.931 & 0.00270 & 0.015000 & -0.030000 \\
\hline-6178.336 & 41.625 & 43.528 & 39.966 & 0.00290 & 0.015000 & -0.029000 \\
\hline-6174.328 & 41.625 & 43.462 & 39.997 & 0.00320 & 0.015000 & -0.028000 \\
\hline-6170.320 & 41.625 & 43.396 & 40.029 & 0.00340 & 0.015000 & -0.027000 \\
\hline-6166.312 & 41.625 & 43.345 & 40.062 & 0.00360 & 0.015000 & -0.026000 \\
\hline-6162.304 & 41.625 & 43.301 & 40.094 & 0.00370 & 0.015000 & -0.026000 \\
\hline-6158.296 & 41.625 & 43.250 & 40.123 & 0.00390 & 0.014000 & -0.025000 \\
\hline-6154.287 & 41.625 & 43.210 & 40.154 & 0.00400 & 0.014000 & -0.024000 \\
\hline-6150.279 & 41.625 & 43.175 & 40.187 & 0.00410 & 0.014000 & -0.023000 \\
\hline
\end{tabular}




\begin{tabular}{|c|c|c|c|c|c|c|}
\hline-6146.271 & 41.625 & 43.140 & 40.222 & 0.00410 & 0.014000 & -0.023000 \\
\hline-6142.263 & 41.625 & 43.112 & 40.254 & 0.00420 & 0.014000 & -0.022000 \\
\hline-6138.255 & 41.625 & 43.089 & 40.289 & 0.00420 & 0.014000 & -0.021000 \\
\hline-6134.247 & 41.875 & 43.073 & 40.320 & 0.00420 & 0.014000 & -0.021000 \\
\hline-6130.239 & 41.875 & 43.053 & 40.352 & 0.00410 & 0.014000 & -0.020000 \\
\hline-6126.231 & 41.875 & 43.042 & 40.387 & 0.00410 & 0.014000 & -0.020000 \\
\hline-6122.223 & 41.875 & 43.029 & 40.419 & 0.00400 & 0.014000 & -0.019000 \\
\hline-6118.214 & 41.875 & 43.020 & 40.449 & 0.00390 & 0.013000 & -0.018000 \\
\hline-6114.206 & 41.875 & 43.013 & 40.483 & 0.00380 & 0.013000 & -0.017000 \\
\hline-6110.198 & 41.875 & 43.008 & 40.517 & 0.00370 & 0.013000 & -0.017000 \\
\hline-6106.190 & 41.875 & 43.004 & 40.552 & 0.00370 & 0.013000 & -0.016000 \\
\hline-6102.182 & 41.875 & 42.998 & 40.587 & 0.00360 & 0.013000 & -0.015000 \\
\hline-6098.174 & 41.875 & 42.994 & 40.621 & 0.00350 & 0.013000 & -0.014000 \\
\hline-6094.166 & 41.875 & 42.989 & 40.654 & 0.00350 & 0.013000 & -0.013000 \\
\hline-6090.158 & 41.875 & 42.987 & 40.687 & 0.00340 & 0.013000 & -0.012000 \\
\hline-6086.149 & 41.875 & 42.985 & 40.719 & 0.00340 & 0.013000 & -0.0100000 \\
\hline-6082.141 & 41.875 & 42.985 & 40.752 & 0.00330 & 0.013000 & -0.0090000 \\
\hline-6078.133 & 41.875 & 42.985 & 40.787 & 0.00330 & 0.013000 & -0.0080000 \\
\hline-6074.125 & 41.875 & 42.988 & 40.819 & 0.00330 & 0.013000 & -0.0070000 \\
\hline-6070.117 & 42.125 & 42.991 & 40.854 & 0.00330 & 0.013000 & -0.0070000 \\
\hline-6066.109 & 42.125 & 42.994 & 40.886 & 0.00330 & 0.013000 & -0.0060000 \\
\hline-6062.101 & 42.125 & 42.999 & 40.919 & 0.00330 & 0.013000 & -0.0060000 \\
\hline-6058.093 & 42.125 & 43.002 & 40.951 & 0.00330 & 0.013000 & -0.0050000 \\
\hline-6054.085 & 42.125 & 43.009 & 40.982 & 0.00330 & 0.013000 & -0.0050000 \\
\hline-6050.076 & 42.125 & 43.014 & 41.016 & 0.00330 & 0.013000 & -0.0050000 \\
\hline-6046.068 & 42.125 & 43.022 & 41.049 & 0.00330 & 0.013000 & -0.0040000 \\
\hline-6042.060 & 42.125 & 43.030 & 41.081 & 0.00340 & 0.013000 & -0.0040000 \\
\hline-6038.052 & 42.125 & 43.037 & 41.112 & 0.00340 & 0.013000 & -0.0040000 \\
\hline-6034.044 & 42.125 & 43.045 & 41.144 & 0.00340 & 0.013000 & -0.0040000 \\
\hline-6030.036 & 42.125 & 43.052 & 41.175 & 0.00350 & 0.013000 & -0.0040000 \\
\hline-6026.028 & 42.125 & 43.061 & 41.206 & 0.00360 & 0.013000 & -0.0040000 \\
\hline-6022.020 & 42.125 & 43.071 & 41.236 & 0.00360 & 0.013000 & -0.0040000 \\
\hline-6018.012 & 42.125 & 43.083 & 41.267 & 0.00370 & 0.013000 & -0.0030000 \\
\hline-6014.003 & 42.125 & 43.095 & 41.296 & 0.00380 & 0.012000 & -0.0030000 \\
\hline-6009.995 & 42.375 & 43.108 & 41.323 & 0.00390 & 0.012000 & -0.0030000 \\
\hline-6005.987 & 42.375 & 43.123 & 41.351 & 0.00390 & 0.012000 & -0.0030000 \\
\hline-6001.979 & 42.375 & 43.135 & 41.379 & 0.00400 & 0.012000 & -0.0030000 \\
\hline-5997.971 & 42.375 & 43.150 & 41.407 & 0.00410 & 0.012000 & -0.0030000 \\
\hline-5993.963 & 42.375 & 43.167 & 41.435 & 0.00420 & 0.012000 & -0.0030000 \\
\hline-5989.955 & 42.375 & 43.183 & 41.462 & 0.00430 & 0.012000 & -0.0030000 \\
\hline-5985.947 & 42.375 & 43.202 & 41.489 & 0.00440 & 0.012000 & -0.0030000 \\
\hline-5981.938 & 42.375 & 43.219 & 41.516 & 0.00450 & 0.012000 & -0.0030000 \\
\hline-5977.930 & 42.375 & 43.238 & 41.541 & 0.00460 & 0.012000 & -0.0030000 \\
\hline-5973.922 & 42.375 & 43.258 & 41.565 & 0.00470 & 0.012000 & -0.0030000 \\
\hline-5969.914 & 42.375 & 43.276 & 41.589 & 0.00480 & 0.012000 & -0.0030000 \\
\hline-5965.906 & 42.375 & 43.298 & 41.611 & 0.00480 & 0.012000 & -0.0030000 \\
\hline-5961.898 & 42.375 & 43.319 & 41.634 & 0.00490 & 0.013000 & -0.0030000 \\
\hline-5957.890 & 42.375 & 43.344 & 41.655 & 0.00490 & 0.013000 & -0.0030000 \\
\hline-5953.882 & 42.375 & 43.373 & 41.676 & 0.00500 & 0.013000 & -0.0020000 \\
\hline-5949.874 & 42.625 & 43.399 & 41.697 & 0.00500 & 0.013000 & -0.0020000 \\
\hline-5945.865 & 42.625 & 43.429 & 41.714 & 0.00510 & 0.013000 & -0.0020000 \\
\hline-5941.857 & 42.625 & 43.462 & 41.732 & 0.00510 & 0.013000 & -0.0020000 \\
\hline-5937.849 & 42.625 & 43.491 & 41.749 & 0.00510 & 0.013000 & -0.0020000 \\
\hline-5933.841 & 42.625 & 43.526 & 41.767 & 0.00520 & 0.013000 & -0.0020000 \\
\hline
\end{tabular}




\begin{tabular}{|c|c|c|c|c|c|c|}
\hline-5929.833 & 42.625 & 43.563 & 41.782 & 0.00520 & 0.013000 & -0.0020000 \\
\hline-5925.825 & 42.625 & 43.599 & 41.799 & 0.00520 & 0.013000 & -0.0020000 \\
\hline-5921.817 & 42.625 & 43.633 & 41.815 & 0.00520 & 0.013000 & -0.0020000 \\
\hline-5917.809 & 42.625 & 43.674 & 41.830 & 0.00520 & 0.013000 & -0.0020000 \\
\hline-5913.801 & 42.625 & 43.714 & 41.847 & 0.00520 & 0.013000 & -0.0020000 \\
\hline-5909.792 & 42.625 & 43.755 & 41.862 & 0.00520 & 0.013000 & -0.0020000 \\
\hline-5905.784 & 42.625 & 43.795 & 41.874 & 0.00520 & 0.013000 & -0.0020000 \\
\hline-5901.776 & 42.625 & 43.838 & 41.886 & 0.00520 & 0.013000 & -0.0020000 \\
\hline-5897.768 & 42.625 & 43.882 & 41.898 & 0.00510 & 0.013000 & -0.0020000 \\
\hline-5893.760 & 42.875 & 43.928 & 41.910 & 0.00510 & 0.013000 & -0.0020000 \\
\hline-5889.752 & 42.875 & 43.971 & 41.918 & 0.00510 & 0.013000 & -0.0020000 \\
\hline-5885.744 & 42.875 & 44.017 & 41.927 & 0.00500 & 0.014000 & -0.0020000 \\
\hline-5881.736 & 42.875 & 44.064 & 41.941 & 0.00500 & 0.014000 & -0.0020000 \\
\hline-5877.727 & 42.875 & 44.110 & 41.954 & 0.00490 & 0.014000 & -0.0020000 \\
\hline-5873.719 & 42.875 & 44.156 & 41.967 & 0.00490 & 0.014000 & -0.0020000 \\
\hline-5869.711 & 42.875 & 44.204 & 41.976 & 0.00480 & 0.014000 & -0.0020000 \\
\hline-5865.703 & 42.875 & 44.252 & 41.988 & 0.00480 & 0.014000 & -0.0020000 \\
\hline-5861.695 & 42.875 & 44.295 & 42.007 & 0.00470 & 0.014000 & -0.0020000 \\
\hline-5857.687 & 42.875 & 44.346 & 42.023 & 0.00460 & 0.014000 & -0.0020000 \\
\hline-5853.679 & 42.875 & 44.393 & 42.044 & 0.00450 & 0.015000 & -0.0020000 \\
\hline-5849.671 & 42.875 & 44.443 & 42.064 & 0.00440 & 0.015000 & -0.0020000 \\
\hline-5845.663 & 42.875 & 44.490 & 42.090 & 0.00430 & 0.015000 & -0.0030000 \\
\hline-5841.654 & 42.875 & 44.538 & 42.123 & 0.00430 & 0.015000 & -0.0030000 \\
\hline-5837.646 & 42.875 & 44.588 & 42.152 & 0.00410 & 0.015000 & -0.0030000 \\
\hline-5833.638 & 43.125 & 44.637 & 42.183 & 0.00400 & 0.015000 & -0.0030000 \\
\hline-5829.630 & 43.125 & 44.688 & 42.219 & 0.00390 & 0.016000 & -0.0030000 \\
\hline-5825.622 & 43.125 & 44.735 & 42.255 & 0.00380 & 0.016000 & -0.0040000 \\
\hline-5821.614 & 43.125 & 44.775 & 42.295 & 0.00370 & 0.016000 & -0.0040000 \\
\hline-5817.606 & 43.125 & 44.816 & 42.337 & 0.00350 & 0.016000 & -0.0050000 \\
\hline-5813.598 & 43.125 & 44.853 & 42.377 & 0.00340 & 0.016000 & -0.0050000 \\
\hline-5809.589 & 43.125 & 44.895 & 42.418 & 0.00330 & 0.016000 & -0.0060000 \\
\hline-5805.581 & 43.125 & 44.933 & 42.458 & 0.00310 & 0.016000 & -0.0070000 \\
\hline-5801.573 & 43.125 & 44.961 & 42.497 & 0.00300 & 0.016000 & -0.0080000 \\
\hline-5797.565 & 43.125 & 44.977 & 42.532 & 0.00280 & 0.016000 & -0.0090000 \\
\hline-5793.557 & 43.125 & 44.996 & 42.565 & 0.00260 & 0.016000 & -0.0100000 \\
\hline-5789.549 & 43.125 & 45.004 & 42.597 & 0.00250 & 0.016000 & -0.012000 \\
\hline-5785.541 & 43.125 & 45.006 & 42.625 & 0.00230 & 0.016000 & -0.013000 \\
\hline-5781.533 & 43.125 & 45.002 & 42.651 & 0.00210 & 0.016000 & -0.015000 \\
\hline-5777.525 & 43.125 & 44.994 & 42.678 & 0.00190 & 0.015000 & -0.016000 \\
\hline-5773.516 & 43.375 & 44.975 & 42.701 & 0.00170 & 0.015000 & -0.018000 \\
\hline-5769.508 & 43.375 & 44.941 & 42.724 & 0.00160 & 0.015000 & -0.020000 \\
\hline-5765.500 & 43.375 & 44.900 & 42.746 & 0.00140 & 0.015000 & -0.022000 \\
\hline-5761.492 & 43.375 & 44.872 & 42.767 & 0.00110 & 0.015000 & -0.023000 \\
\hline-5757.484 & 43.375 & 44.842 & 42.786 & 0.000900 & 0.015000 & -0.025000 \\
\hline-5753.476 & 43.375 & 44.804 & 42.805 & 0.000700 & 0.015000 & -0.028000 \\
\hline-5749.468 & 43.375 & 44.756 & 42.822 & 0.000500 & 0.015000 & -0.030000 \\
\hline-5745.460 & 43.375 & 44.722 & 42.835 & 0.000300 & 0.015000 & -0.032000 \\
\hline-5741.452 & 43.375 & 44.674 & 42.845 & 0.000200 & 0.014000 & -0.034000 \\
\hline-5737.443 & 43.375 & 44.639 & 42.850 & 0.000200 & 0.014000 & -0.037000 \\
\hline-5733.435 & 43.375 & 44.616 & 42.851 & 0.000400 & 0.014000 & -0.041000 \\
\hline-5729.427 & 43.375 & 44.578 & 42.845 & 0.000700 & 0.014000 & -0.044000 \\
\hline-5725.419 & 43.375 & 44.558 & 42.828 & 0.00120 & 0.014000 & -0.048000 \\
\hline-5721.411 & 43.375 & 44.543 & 42.794 & 0.00190 & 0.014000 & -0.052000 \\
\hline-5717.403 & 43.375 & 44.525 & 42.729 & 0.00270 & 0.014000 & -0.057000 \\
\hline
\end{tabular}




\begin{tabular}{|c|c|c|c|c|c|c|}
\hline-5713.395 & 43.375 & 44.515 & 42.626 & 0.00370 & 0.014000 & -0.061000 \\
\hline-5709.387 & 43.375 & 44.505 & 42.473 & 0.00490 & 0.013000 & -0.065000 \\
\hline-5705.378 & 43.375 & 44.501 & 42.285 & 0.00620 & 0.013000 & -0.070000 \\
\hline-5701.370 & 43.625 & 44.502 & 42.078 & 0.00770 & 0.013000 & -0.076000 \\
\hline-5697.362 & 43.625 & 44.506 & 41.860 & 0.00940 & 0.013000 & -0.082000 \\
\hline-5693.354 & 43.625 & 44.518 & 41.609 & 0.0112 & 0.013000 & -0.089000 \\
\hline-5689.346 & 43.625 & 44.525 & 41.338 & 0.0132 & 0.013000 & -0.096000 \\
\hline-5685.338 & 43.625 & 44.531 & 41.050 & 0.0154 & 0.012000 & -0.10400 \\
\hline-5681.330 & 43.625 & 44.533 & 40.740 & 0.0176 & 0.012000 & -0.11300 \\
\hline-5677.322 & 43.625 & 44.540 & 40.417 & 0.0192 & 0.012000 & -0.12300 \\
\hline-5673.314 & 43.625 & 44.547 & 40.052 & 0.0194 & 0.011000 & -0.13400 \\
\hline-5669.305 & 43.625 & 44.553 & 39.658 & 0.0182 & 0.011000 & -0.14600 \\
\hline-5665.297 & 43.625 & 44.558 & 39.255 & 0.0156 & 0.011000 & -0.15900 \\
\hline-5661.289 & 43.625 & 44.565 & 38.829 & 0.0116 & 0.011000 & -0.17200 \\
\hline-5657.281 & 43.625 & 44.573 & 38.373 & 0.00610 & 0.011000 & -0.18800 \\
\hline-5653.273 & 43.625 & 44.578 & 37.871 & -0.000900 & 0.0100000 & -0.20700 \\
\hline-5649.265 & 43.625 & 44.588 & 37.346 & -0.00920 & 0.0100000 & -0.22800 \\
\hline-5645.257 & 43.625 & 44.597 & 36.779 & -0.0190 & 0.0100000 & -0.25100 \\
\hline-5641.249 & 43.375 & 44.599 & 36.147 & -0.0303 & 0.0100000 & -0.27900 \\
\hline-5637.240 & 43.375 & 44.602 & 35.420 & -0.0429 & 0.0100000 & -0.31100 \\
\hline-5633.232 & 43.375 & 44.599 & 34.603 & -0.0571 & 0.0100000 & -0.34600 \\
\hline-5629.224 & 43.375 & 44.601 & 33.660 & -0.0726 & 0.011000 & -0.38500 \\
\hline-5625.216 & 43.375 & 44.602 & 32.649 & -0.0896 & 0.012000 & -0.43300 \\
\hline-5621.208 & 43.125 & 44.590 & 31.615 & -0.108 & 0.015000 & -0.48200 \\
\hline-5617.200 & 42.625 & 44.583 & 30.550 & -0.124 & 0.026000 & -0.53200 \\
\hline-5613.192 & 42.125 & 44.568 & 29.555 & -0.138 & 0.088000 & -0.58200 \\
\hline-5609.184 & 41.625 & 44.549 & 28.684 & -0.148 & 0.15700 & -0.63500 \\
\hline-5605.176 & 40.875 & 44.530 & 28.051 & -0.154 & 0.21500 & -0.69300 \\
\hline-5601.167 & 39.875 & 44.512 & 27.666 & -0.156 & 0.28300 & -0.74300 \\
\hline-5597.159 & 39.125 & 44.494 & 27.317 & -0.155 & 0.36600 & -0.77400 \\
\hline-5593.151 & 38.375 & 44.475 & 26.985 & -0.150 & 0.47200 & -0.78600 \\
\hline-5589.143 & 37.625 & 44.469 & 26.682 & -0.142 & 0.59800 & -0.79100 \\
\hline-5585.135 & 37.125 & 44.514 & 26.345 & -0.129 & 0.72600 & -0.77800 \\
\hline-5581.127 & 36.625 & 44.616 & 26.086 & -0.112 & 0.84500 & -0.76500 \\
\hline-5577.119 & 36.125 & 44.949 & 25.960 & -0.0916 & 0.95600 & -0.75200 \\
\hline-5573.111 & 35.875 & 45.645 & 25.818 & -0.0668 & 1.0420 & -0.73700 \\
\hline-5569.103 & 35.875 & 46.253 & 25.723 & -0.0378 & 1.0970 & -0.72400 \\
\hline-5565.094 & 35.875 & 46.737 & 25.665 & -0.00470 & 1.1570 & -0.69900 \\
\hline-5561.086 & 35.875 & 47.236 & 25.625 & 0.0325 & 1.1960 & -0.68100 \\
\hline-5557.078 & 36.125 & 47.584 & 25.550 & 0.0726 & 1.2460 & -0.66300 \\
\hline-5553.070 & 36.375 & 47.862 & 25.562 & 0.110 & 1.2860 & -0.63500 \\
\hline-5549.062 & 36.625 & 48.177 & 25.624 & 0.139 & 1.3020 & -0.60200 \\
\hline-5545.054 & 37.125 & 48.488 & 25.659 & 0.159 & 1.3050 & -0.57000 \\
\hline-5541.046 & 37.625 & 48.737 & 25.784 & 0.170 & 1.3090 & -0.54100 \\
\hline-5537.038 & 38.375 & 48.906 & 25.873 & 0.171 & 1.2790 & -0.51000 \\
\hline-5533.029 & 39.375 & 49.094 & 25.996 & 0.163 & 1.2480 & -0.47700 \\
\hline-5529.021 & 40.375 & 49.176 & 26.162 & 0.146 & 1.2330 & -0.44200 \\
\hline-5525.013 & 41.125 & 49.244 & 26.369 & 0.120 & 1.2200 & -0.41800 \\
\hline-5521.005 & 41.625 & 49.315 & 26.553 & 0.0915 & 1.1670 & -0.38900 \\
\hline-5516.997 & 41.875 & 49.351 & 26.794 & 0.0654 & 1.1220 & -0.36900 \\
\hline-5512.989 & 42.125 & 49.311 & 27.026 & 0.0430 & 1.1010 & -0.34900 \\
\hline-5508.981 & 42.125 & 49.301 & 27.290 & 0.0242 & 1.0470 & -0.33200 \\
\hline-5504.973 & 42.125 & 49.212 & 27.669 & 0.00900 & 0.99200 & -0.31700 \\
\hline-5500.965 & 42.125 & 49.130 & 28.020 & -0.00250 & 0.92100 & -0.30200 \\
\hline
\end{tabular}




\begin{tabular}{|c|c|c|c|c|c|c|}
\hline-5496.956 & 42.125 & 49.040 & 28.374 & -0.0107 & 0.86100 & -0.29200 \\
\hline-5492.948 & 42.125 & 48.889 & 28.771 & -0.0170 & 0.80600 & -0.28000 \\
\hline-5488.940 & 42.125 & 48.757 & 29.192 & -0.0227 & 0.74500 & -0.27000 \\
\hline-5484.932 & 41.875 & 48.611 & 29.668 & -0.0280 & 0.69400 & -0.26300 \\
\hline-5480.924 & 41.625 & 48.449 & 30.220 & -0.0331 & 0.63400 & -0.25700 \\
\hline-5476.916 & 41.625 & 48.247 & 30.846 & -0.0379 & 0.58200 & -0.25300 \\
\hline-5472.908 & 41.375 & 48.049 & 31.485 & -0.0423 & 0.52900 & -0.24800 \\
\hline-5468.900 & 41.125 & 47.811 & 32.080 & -0.0465 & 0.47500 & -0.24300 \\
\hline-5464.891 & 40.875 & 47.539 & 32.521 & -0.0503 & 0.43600 & -0.23700 \\
\hline-5460.883 & 40.625 & 47.271 & 32.841 & -0.0538 & 0.39800 & -0.23400 \\
\hline-5456.875 & 40.375 & 46.990 & 33.072 & -0.0571 & 0.36000 & -0.23000 \\
\hline-5452.867 & 40.125 & 46.704 & 33.164 & -0.0600 & 0.32700 & -0.22800 \\
\hline-5448.859 & 39.875 & 46.429 & 33.219 & -0.0626 & 0.29600 & -0.22300 \\
\hline-5444.851 & 39.625 & 46.173 & 33.226 & -0.0649 & 0.27000 & -0.22000 \\
\hline-5440.843 & 39.375 & 45.932 & 33.194 & -0.0669 & 0.24000 & -0.21700 \\
\hline-5436.835 & 38.875 & 45.669 & 33.135 & -0.0686 & 0.21400 & -0.21400 \\
\hline-5432.827 & 38.625 & 45.391 & 33.082 & -0.0700 & 0.18900 & -0.21100 \\
\hline-5428.818 & 38.375 & 45.096 & 33.018 & -0.0711 & 0.15900 & -0.20700 \\
\hline-5424.810 & 38.125 & 44.807 & 32.944 & -0.0719 & 0.13300 & -0.20400 \\
\hline-5420.802 & 37.875 & 44.542 & 32.878 & -0.0724 & 0.098000 & -0.20000 \\
\hline-5416.794 & 37.625 & 44.267 & 32.802 & -0.0726 & 0.030000 & -0.19700 \\
\hline-5412.786 & 37.125 & 44.000 & 32.730 & -0.0724 & 0.0060000 & -0.19200 \\
\hline-5408.778 & 36.875 & 43.715 & 32.664 & -0.0720 & -0.0020000 & -0.18800 \\
\hline-5404.770 & 36.625 & 43.432 & 32.594 & -0.0713 & -0.0030000 & -0.18300 \\
\hline-5400.762 & 36.375 & 43.175 & 32.531 & -0.0702 & -0.0040000 & -0.17800 \\
\hline-5396.754 & 36.125 & 42.905 & 32.466 & -0.0689 & -0.0040000 & -0.17300 \\
\hline-5392.745 & 35.875 & 42.670 & 32.400 & -0.0672 & -0.0040000 & -0.16800 \\
\hline-5388.737 & 35.625 & 42.418 & 32.340 & -0.0653 & -0.0040000 & -0.16400 \\
\hline-5384.729 & 35.375 & 42.157 & 32.286 & -0.0630 & -0.0040000 & -0.16000 \\
\hline-5380.721 & 35.125 & 41.917 & 32.229 & -0.0604 & -0.0040000 & -0.15600 \\
\hline-5376.713 & 34.875 & 41.671 & 32.166 & -0.0575 & -0.0040000 & -0.15100 \\
\hline-5372.705 & 34.625 & 41.391 & 32.110 & -0.0544 & -0.0040000 & -0.14700 \\
\hline-5368.697 & 34.625 & 41.133 & 32.051 & -0.0512 & -0.0040000 & -0.14300 \\
\hline-5364.689 & 34.375 & 40.850 & 31.990 & -0.0480 & -0.0030000 & -0.13900 \\
\hline-5360.680 & 34.125 & 40.579 & 31.930 & -0.0450 & -0.0030000 & -0.13500 \\
\hline-5356.672 & 34.125 & 40.295 & 31.865 & -0.0421 & -0.0030000 & -0.13200 \\
\hline-5352.664 & 33.875 & 40.015 & 31.803 & -0.0393 & -0.0030000 & -0.12800 \\
\hline-5348.656 & 33.875 & 39.727 & 31.744 & -0.0366 & -0.0030000 & -0.12500 \\
\hline-5344.648 & 33.625 & 39.458 & 31.678 & -0.0340 & -0.0020000 & -0.12100 \\
\hline-5340.640 & 33.625 & 39.155 & 31.611 & -0.0316 & -0.0020000 & -0.11700 \\
\hline-5336.632 & 33.625 & 38.832 & 31.545 & -0.0293 & -0.0020000 & -0.11400 \\
\hline-5332.624 & 33.375 & 38.516 & 31.478 & -0.0271 & -0.0010000 & -0.11100 \\
\hline-5328.616 & 33.375 & 38.223 & 31.406 & -0.0250 & -0.0010000 & -0.10700 \\
\hline-5324.607 & 33.125 & 37.900 & 31.337 & -0.0230 & -0.0010000 & -0.10400 \\
\hline-5320.599 & 33.125 & 37.608 & 31.269 & -0.0212 & 0.0000 & -0.10100 \\
\hline-5316.591 & 33.125 & 37.340 & 31.205 & -0.0194 & 0.0000 & -0.098000 \\
\hline-5312.583 & 32.875 & 37.074 & 31.139 & -0.0178 & 0.0010000 & -0.095000 \\
\hline-5308.575 & 32.875 & 36.805 & 31.068 & -0.0164 & 0.0010000 & -0.092000 \\
\hline-5304.567 & 32.875 & 36.507 & 30.992 & -0.0150 & 0.0010000 & -0.089000 \\
\hline-5300.559 & 32.875 & 36.227 & 30.923 & -0.0137 & 0.0020000 & -0.086000 \\
\hline-5296.551 & 32.625 & 35.926 & 30.864 & -0.0126 & 0.0020000 & -0.083000 \\
\hline-5292.542 & 32.625 & 35.636 & 30.795 & -0.0116 & 0.0020000 & -0.081000 \\
\hline-5288.534 & 32.625 & 35.381 & 30.730 & -0.0107 & 0.0030000 & -0.078000 \\
\hline-5284.526 & 32.625 & 35.148 & 30.676 & -0.00990 & 0.0030000 & -0.075000 \\
\hline
\end{tabular}




\begin{tabular}{|c|c|c|c|c|c|c|}
\hline-5280.518 & 32.375 & 34.933 & 30.625 & -0.00930 & 0.0040000 & -0.072000 \\
\hline-5276.510 & 32.375 & 34.739 & 30.574 & -0.00880 & 0.0040000 & -0.069000 \\
\hline-5272.502 & 32.375 & 34.553 & 30.522 & -0.00830 & 0.0040000 & -0.065000 \\
\hline-5268.494 & 32.375 & 34.390 & 30.471 & -0.00800 & 0.0050000 & -0.062000 \\
\hline-5264.486 & 32.125 & 34.247 & 30.434 & -0.00790 & 0.0050000 & -0.059000 \\
\hline-5260.478 & 32.125 & 34.118 & 30.396 & -0.00780 & 0.0060000 & -0.056000 \\
\hline-5256.469 & 32.125 & 33.996 & 30.349 & -0.00790 & 0.0060000 & -0.052000 \\
\hline-5252.461 & 32.125 & 33.887 & 30.311 & -0.00810 & 0.0060000 & -0.050000 \\
\hline-5248.453 & 32.125 & 33.781 & 30.273 & -0.00840 & 0.0070000 & -0.047000 \\
\hline-5244.445 & 31.875 & 33.684 & 30.239 & -0.00870 & 0.0070000 & -0.045000 \\
\hline-5240.437 & 31.875 & 33.589 & 30.202 & -0.00900 & 0.0070000 & -0.042000 \\
\hline-5236.429 & 31.875 & 33.500 & 30.167 & -0.00940 & 0.0080000 & -0.040000 \\
\hline-5232.421 & 31.875 & 33.414 & 30.132 & -0.00970 & 0.0080000 & -0.038000 \\
\hline-5228.413 & 31.875 & 33.333 & 30.104 & -0.01000 & 0.0080000 & -0.036000 \\
\hline-5224.405 & 31.625 & 33.251 & 30.064 & -0.0102 & 0.0090000 & -0.035000 \\
\hline-5220.396 & 31.625 & 33.170 & 30.028 & -0.0105 & 0.0090000 & -0.034000 \\
\hline-5216.388 & 31.625 & 33.094 & 29.988 & -0.0107 & 0.0090000 & -0.033000 \\
\hline-5212.380 & 31.625 & 33.019 & 29.949 & -0.0110 & 0.0100000 & -0.032000 \\
\hline-5208.372 & 31.625 & 32.946 & 29.904 & -0.0112 & 0.0100000 & -0.031000 \\
\hline-5204.364 & 31.625 & 32.876 & 29.864 & -0.0114 & 0.0100000 & -0.030000 \\
\hline-5200.356 & 31.375 & 32.809 & 29.823 & -0.0116 & 0.011000 & -0.030000 \\
\hline-5196.348 & 31.375 & 32.741 & 29.774 & -0.0118 & 0.011000 & -0.029000 \\
\hline-5192.340 & 31.375 & 32.674 & 29.729 & -0.0119 & 0.011000 & -0.029000 \\
\hline-5188.331 & 31.375 & 32.611 & 29.685 & -0.0120 & 0.012000 & -0.028000 \\
\hline-5184.323 & 31.375 & 32.547 & 29.640 & -0.0122 & 0.012000 & -0.028000 \\
\hline-5180.315 & 31.375 & 32.489 & 29.593 & -0.0123 & 0.012000 & -0.028000 \\
\hline-5176.307 & 31.125 & 32.432 & 29.544 & -0.0124 & 0.013000 & -0.027000 \\
\hline-5172.299 & 31.125 & 32.378 & 29.494 & -0.0125 & 0.013000 & -0.027000 \\
\hline-5168.291 & 31.125 & 32.324 & 29.446 & -0.0125 & 0.014000 & -0.027000 \\
\hline-5164.283 & 31.125 & 32.274 & 29.399 & -0.0126 & 0.015000 & -0.027000 \\
\hline-5160.275 & 31.125 & 32.225 & 29.349 & -0.0126 & 0.015000 & -0.026000 \\
\hline-5156.267 & 31.125 & 32.178 & 29.303 & -0.0126 & 0.016000 & -0.026000 \\
\hline-5152.258 & 30.875 & 32.137 & 29.253 & -0.0126 & 0.016000 & -0.026000 \\
\hline-5148.250 & 30.875 & 32.099 & 29.207 & -0.0126 & 0.017000 & -0.026000 \\
\hline-5144.242 & 30.875 & 32.066 & 29.161 & -0.0126 & 0.018000 & -0.026000 \\
\hline-5140.234 & 30.875 & 32.033 & 29.112 & -0.0125 & 0.019000 & -0.026000 \\
\hline-5136.226 & 30.875 & 32.003 & 29.065 & -0.0125 & 0.020000 & -0.026000 \\
\hline-5132.218 & 30.875 & 31.977 & 29.012 & -0.0124 & 0.021000 & -0.026000 \\
\hline-5128.210 & 30.625 & 31.957 & 28.971 & -0.0123 & 0.022000 & -0.025000 \\
\hline-5124.202 & 30.625 & 31.944 & 28.924 & -0.0122 & 0.023000 & -0.025000 \\
\hline-5120.193 & 30.625 & 31.933 & 28.875 & -0.0121 & 0.024000 & -0.025000 \\
\hline-5116.185 & 30.625 & 31.931 & 28.824 & -0.0120 & 0.026000 & -0.025000 \\
\hline-5112.177 & 30.625 & 31.940 & 28.777 & -0.0118 & 0.027000 & -0.025000 \\
\hline-5108.169 & 30.625 & 31.953 & 28.726 & -0.0117 & 0.029000 & -0.025000 \\
\hline-5104.161 & 30.375 & 31.976 & 28.676 & -0.0115 & 0.031000 & -0.025000 \\
\hline-5100.153 & 30.375 & 32.011 & 28.626 & -0.0113 & 0.033000 & -0.025000 \\
\hline-5096.145 & 30.375 & 32.062 & 28.572 & -0.0111 & 0.036000 & -0.026000 \\
\hline-5092.137 & 30.375 & 32.116 & 28.521 & -0.0108 & 0.039000 & -0.026000 \\
\hline-5088.129 & 30.375 & 32.182 & 28.463 & -0.0106 & 0.042000 & -0.026000 \\
\hline-5084.120 & 30.375 & 32.261 & 28.409 & -0.0104 & 0.045000 & -0.026000 \\
\hline-5080.112 & 30.125 & 32.342 & 28.349 & -0.0101 & 0.049000 & -0.026000 \\
\hline-5076.104 & 30.125 & 32.444 & 28.288 & -0.00980 & 0.053000 & -0.027000 \\
\hline-5072.096 & 30.125 & 32.562 & 28.223 & -0.00950 & 0.058000 & -0.027000 \\
\hline-5068.088 & 30.125 & 32.695 & 28.160 & -0.00920 & 0.064000 & -0.027000 \\
\hline
\end{tabular}




$\begin{array}{lllllll}-5064.080 & 30.125 & 32.846 & 28.094 & -0.00880 & 0.071000 & -0.028000 \\ -5060.072 & 30.125 & 33.011 & 28.029 & -0.00850 & 0.080000 & -0.029000 \\ -5056.064 & 29.875 & 33.236 & 27.961 & -0.00810 & 0.090000 & -0.030000 \\ -5052.056 & 29.875 & 33.480 & 27.887 & -0.00780 & 0.10400 & -0.031000 \\ -5048.047 & 29.875 & 33.778 & 27.817 & -0.00740 & 0.12300 & -0.033000 \\ -5044.039 & 29.875 & 34.187 & 27.738 & -0.00700 & 0.14800 & -0.035000 \\ -5040.031 & 29.875 & 34.675 & 27.656 & -0.00650 & 0.17700 & -0.039000 \\ -5036.023 & 29.875 & 35.313 & 27.570 & -0.00610 & 0.21700 & -0.043000 \\ -5032.015 & 29.875 & 36.160 & 27.481 & -0.00560 & 0.26700 & -0.047000 \\ -5028.007 & 29.625 & 37.240 & 27.377 & -0.00520 & 0.33100 & -0.053000 \\ -5023.999 & 29.625 & 38.628 & 27.268 & -0.00470 & 0.39900 & -0.061000 \\ -5019.991 & 29.625 & 40.312 & 27.145 & -0.00420 & 0.49100 & -0.069000 \\ -5015.982 & 29.625 & 42.364 & 27.008 & -0.00370 & 0.61000 & -0.079000 \\ -5011.974 & 29.625 & 44.856 & 26.837 & -0.00310 & 0.76900 & -0.095000 \\ -5007.966 & 29.625 & 48.086 & 26.630 & -0.00260 & 1.0090 & -0.11200 \\ -5003.958 & 29.625 & 52.016 & 26.325 & -0.00200 & 1.4140 & -0.13600 \\ -4999.950 & 29.625 & 59.894 & 25.640 & -0.00150 & 2.3370 & -0.17400\end{array}$


(All data transferred to the latitude of Tell Halaf, Syria)

\begin{tabular}{|c|c|c|c|c|c|}
\hline \multicolumn{2}{|c|}{ Data Fig. S4a,b } & \multirow[b]{2}{*}{$\mathrm{dt}$} & \multirow[b]{2}{*}{ Intensity } & \multirow[b]{2}{*}{ sd } & \multirow[b]{2}{*}{ Order } \\
\hline Ref. & Date & & & & \\
\hline SY127 & -6825.0 & 175.00 & 55.066 & 1.7083 & $1 \mathrm{a}$ \\
\hline SY125 & -6675.0 & 50.000 & 53.057 & 2.8136 & $2 a$ \\
\hline SY98-128 & -6505.0 & 195.00 & 50.444 & 3.7180 & $3 a$ \\
\hline SY97-129 & -6505.0 & 195.00 & 52.755 & 3.3160 & $4 a$ \\
\hline SY96 & -6505.0 & 195.00 & 48.334 & 3.0146 & $5 a$ \\
\hline SY130 & -6175.0 & 185.00 & 45.219 & 3.8185 & $6 a$ \\
\hline SY94-137 & -5957.5 & 82.500 & 46.023 & 1.8088 & $7 a$ \\
\hline SY95 & -5957.5 & 82.500 & 42.908 & 0.90438 & $7 a$ \\
\hline SY91 & -5825.0 & 100.00 & 42.707 & 4.1199 & $8 a$ \\
\hline SY87 & -5662.5 & 112.50 & 40.998 & 1.6078 & $9 a$ \\
\hline SY88 & -5662.5 & 112.50 & 42.606 & 1.5073 & $9 a$ \\
\hline SY89 & -5662.5 & 112.50 & 40.998 & 1.9092 & $9 a$ \\
\hline SY90 & -5662.5 & 112.50 & 41.601 & 2.3112 & $9 a$ \\
\hline SY86-131 & -5437.5 & 162.50 & 30.950 & 3.5170 & $10 a$ \\
\hline SY135 & -5437.5 & 162.50 & 45.520 & 2.1102 & $11 a$ \\
\hline SY84 & -5437.5 & 162.50 & 40.496 & 2.4117 & $12 a$ \\
\hline SY138 & -5437.5 & 162.50 & 41.601 & 2.3112 & $12 \mathrm{a}$ \\
\hline SY82 & -5437.5 & 162.50 & 38.486 & 2.8136 & $13 a$ \\
\hline SY83-136 & -5437.5 & 162.50 & 39.089 & 1.7083 & $13 a$ \\
\hline SY80 & -5437.5 & 162.50 & 35.773 & 3.5170 & $14 a$ \\
\hline SY81 & -5437.5 & 162.50 & 36.276 & 2.0097 & $14 a$ \\
\hline SY132 & -5437.5 & 162.50 & 40.596 & 2.9141 & $14 a$ \\
\hline SY37 & -5212.5 & 212.50 & 29.662 & 1.8539 & 0 \\
\hline SY38 & -5212.5 & 212.50 & 28.632 & 0.92693 & 0 \\
\hline YT29 & -5675.0 & 125.00 & 43.352 & 0.20117 & $1 b$ \\
\hline YT28 & -5675.0 & 125.00 & 46.671 & 1.6093 & $1 b$ \\
\hline YT27 & -5675.0 & 125.00 & 45.967 & 2.1123 & $1 b$ \\
\hline YT26 & -5675.0 & 125.00 & 45.062 & 0.90526 & $2 b$ \\
\hline YT25 & -5675.0 & 125.00 & 43.352 & 0.80467 & $3 b$ \\
\hline YT24 & -5675.0 & 125.00 & 44.056 & 5.0292 & $3 b$ \\
\hline YT23 & -5675.0 & 125.00 & 39.429 & 4.5263 & $4 b$ \\
\hline YT22A & -5675.0 & 125.00 & 31.181 & 2.3134 & $4 b$ \\
\hline YT22 & -5437.5 & 162.50 & 44.559 & 1.4082 & $5 b$ \\
\hline YT21 & -5437.5 & 162.50 & 36.110 & 1.3076 & $6 b$ \\
\hline YT20 & -5437.5 & 162.50 & 32.690 & 1.2070 & $7 b$ \\
\hline YT19 & -5437.5 & 162.50 & 32.790 & 1.1064 & $7 b$ \\
\hline YT17 & -5437.5 & 162.50 & 32.690 & 1.5088 & $7 b$ \\
\hline YT16 & -5437.5 & 162.50 & 31.885 & 1.0058 & $7 b$ \\
\hline YT15 & -5437.5 & 162.50 & 32.589 & 1.5088 & $7 b$ \\
\hline YT14 & -5437.5 & 162.50 & 32.589 & 1.6093 & $8 b$ \\
\hline YT11 & -5437.5 & 162.50 & 32.086 & 1.1064 & $9 b$ \\
\hline YT10 & -5437.5 & 162.50 & 30.980 & 0.60350 & $9 b$ \\
\hline YT07 & -5212.5 & 212.50 & 33.494 & 2.8163 & $10 \mathrm{~b}$ \\
\hline YT06 & -5212.5 & 212.50 & 29.874 & 2.0117 & $11 b$ \\
\hline YT04 & -5212.5 & 212.50 & 31.583 & 3.4199 & $12 b$ \\
\hline YT03 & -5212.5 & 212.50 & 32.086 & 1.4082 & $13 b$ \\
\hline YT01 & -5212.5 & 212.50 & 31.684 & 2.1123 & $14 b$ \\
\hline YTI-12 & -6050.0 & 250.00 & 40.636 & 1.4082 & $1 c$ \\
\hline YTI-11 & -6050.0 & 250.00 & 42.849 & 2.2129 & $2 c$ \\
\hline YTI-10 & -6050.0 & 250.00 & 40.837 & 1.7099 & $3 c$ \\
\hline YTI-08A & -6050.0 & 250.00 & 44.358 & 3.4199 & $4 c$ \\
\hline YTI-06 & -6050.0 & 250.00 & 42.245 & 0.70409 & $5 c$ \\
\hline YTI-05 & -6050.0 & 250.00 & 41.340 & 1.2070 & $5 c$ \\
\hline YTI-03 & -6050.0 & 250.00 & 45.162 & 2.2129 & $6 c$ \\
\hline YTI-02A & -6050.0 & 250.00 & 41.139 & 0.70409 & $7 c$ \\
\hline YTI-01 & -6050.0 & 250.00 & 41.843 & 3.0175 & $7 c$ \\
\hline TellBegum & -5400.0 & 75.000 & 33.917 & 1.2186 & 0 \\
\hline
\end{tabular}

\begin{tabular}{|c|c|c|c|c|}
\hline \multicolumn{2}{|c|}{ Data Fig. S4c,d } & \multirow[b]{2}{*}{ Intensity } & \multirow[b]{2}{*}{ sd } & \multirow[b]{2}{*}{ Order } \\
\hline Date & $\mathrm{dt}$ & & & \\
\hline-6825.0 & 175.00 & 55.066 & 1.7083 & $1 a$ \\
\hline-6675.0 & 50.000 & 53.057 & 2.8136 & $2 a$ \\
\hline-6505.0 & 195.00 & 50.444 & 3.7180 & 3a \\
\hline-6505.0 & 195.00 & 52.755 & 3.3160 & $4 a$ \\
\hline-6505.0 & 195.00 & 48.334 & 3.0146 & $5 a$ \\
\hline-6175.0 & 185.00 & 45.219 & 3.8185 & $6 a$ \\
\hline-5957.5 & 82.500 & 46.023 & 1.8088 & $7 a$ \\
\hline-5957.5 & 82.500 & 42.908 & 0.90438 & $7 a$ \\
\hline-5825.0 & 100.00 & 42.707 & 4.1199 & $8 a$ \\
\hline-5662.5 & 112.50 & 40.998 & 1.6078 & $9 a$ \\
\hline-5662.5 & 112.50 & 42.606 & 1.5073 & $9 a$ \\
\hline-5662.5 & 112.50 & 40.998 & 1.9092 & $9 a$ \\
\hline-5662.5 & 112.50 & 41.601 & 2.3112 & $9 a$ \\
\hline-5437.5 & 162.50 & 30.950 & 3.5170 & $10 a$ \\
\hline-5437.5 & 162.50 & 45.520 & 2.1102 & $11 a$ \\
\hline-5437.5 & 162.50 & 40.496 & 2.4117 & $12 a$ \\
\hline-5437.5 & 162.50 & 41.601 & 2.3112 & $12 a$ \\
\hline-5437.5 & 162.50 & 38.486 & 2.8136 & $13 a$ \\
\hline-5437.5 & 162.50 & 39.089 & 1.7083 & $13 a$ \\
\hline-5437.5 & 162.50 & 35.773 & 3.5170 & $14 a$ \\
\hline-5437.5 & 162.50 & 36.276 & 2.0097 & $14 a$ \\
\hline-5437.5 & 162.50 & 40.596 & 2.9141 & $14 a$ \\
\hline-5212.5 & 212.50 & 29.662 & 1.8539 & 0 \\
\hline-5212.5 & 212.50 & 28.632 & 0.92693 & 0 \\
\hline-5400.0 & 400.00 & 43.352 & 0.20117 & $1 b$ \\
\hline-5400.0 & 400.00 & 46.671 & 1.6093 & $1 b$ \\
\hline-5400.0 & 400.00 & 45.967 & 2.1123 & $1 b$ \\
\hline-5400.0 & 400.00 & 45.062 & 0.90526 & $1 b$ \\
\hline-5400.0 & 400.00 & 43.352 & 0.80467 & $2 b$ \\
\hline-5400.0 & 400.00 & 44.056 & 5.0292 & $2 b$ \\
\hline-5400.0 & 400.00 & 39.429 & 4.5263 & $2 b$ \\
\hline-5400.0 & 400.00 & 31.181 & 2.3134 & $3 b$ \\
\hline-5400.0 & 400.00 & 44.559 & 1.4082 & $4 b$ \\
\hline-5400.0 & 400.00 & 36.110 & 1.3076 & $4 b$ \\
\hline-5400.0 & 400.00 & 32.690 & 1.2070 & $4 b$ \\
\hline-5400.0 & 400.00 & 32.790 & 1.1064 & $4 b$ \\
\hline-5400.0 & 400.00 & 32.690 & 1.5088 & $5 b$ \\
\hline-5400.0 & 400.00 & 31.885 & 1.0058 & $5 b$ \\
\hline-5400.0 & 400.00 & 32.589 & 1.5088 & $5 b$ \\
\hline-5400.0 & 400.00 & 32.589 & 1.6093 & $5 b$ \\
\hline-5400.0 & 400.00 & 32.086 & 1.1064 & $6 b$ \\
\hline-5400.0 & 400.00 & 30.980 & 0.60350 & $6 b$ \\
\hline-5400.0 & 400.00 & 33.494 & 2.8163 & $7 b$ \\
\hline-5400.0 & 400.00 & 29.874 & 2.0117 & $7 b$ \\
\hline-5400.0 & 400.00 & 31.583 & 3.4199 & $8 b$ \\
\hline-5400.0 & 400.00 & 32.086 & 1.4082 & $8 b$ \\
\hline-5400.0 & 400.00 & 31.684 & 2.1123 & $9 b$ \\
\hline-6050.0 & 250.00 & 40.636 & 1.4082 & $1 c$ \\
\hline-6050.0 & 250.00 & 42.849 & 2.2129 & $2 c$ \\
\hline-6050.0 & 250.00 & 40.837 & 1.7099 & $3 c$ \\
\hline-6050.0 & 250.00 & 44.358 & 3.4199 & $4 c$ \\
\hline-6050.0 & 250.00 & 42.245 & 0.70409 & $5 c$ \\
\hline-6050.0 & 250.00 & 41.340 & 1.2070 & $5 c$ \\
\hline-6050.0 & 250.00 & 45.162 & 2.2129 & $6 c$ \\
\hline-6050.0 & 250.00 & 41.139 & 0.70409 & $7 c$ \\
\hline-6050.0 & 250.00 & 41.843 & 3.0175 & $8 c$ \\
\hline-5400.0 & 75.000 & 33.917 & 1.2186 & 0 \\
\hline
\end{tabular}

